Supporting Information for:

\title{
Detosylative (Deutero)alkylation of Indoles and Phenols with (Deutero)alkoxides
}

Ming-Hui Zhu, ${ }^{\dagger}$ Cheng-Long Yu, ${ }^{\dagger}$ Ya-Lan Feng,${ }^{\dagger}$ Muhammad Usman, ${ }^{\dagger}$ Dayou Zhong, ${ }^{\dagger}$ Xin Wang, ${ }^{\dagger}$ Nasri Nesnas, ${ }^{\ddagger}$ and Wen-Bo Liu ${ }^{*}{ }^{\dagger}$

\section{Email: wenboliu@whu.edu.cn}

${ }^{\dagger}$ Engineering Research Center of Organosilicon Compounds \& Materials, Ministry of Education, Sauvage Center for Molecular Sciences, College of Chemistry and Molecular Sciences, Wuhan University, 299 Bayi Road, Wuhan, Hubei 430072, China

Department of Biomedical and Chemical Engineering and Sciences, Florida Institute of Technology, Melbourne, Florida 32901, United States

\section{Table of Contents}

General Information and Materials 2

Scheme S1. Debrominative Deuteration vs Detosylative Methylation. ........................................... 3

Table S1. Reaction Condition Optimizations for N-Methylation of $N$-Tosylindole. .......................... 3

Table S2. Reaction Condition Optimizations for O-Methylation of $O$-Tosylphenol. ....................... 4

General Procedure and Spectroscopic Data for Detosylation/(Deutero)alkylation of Indoles and

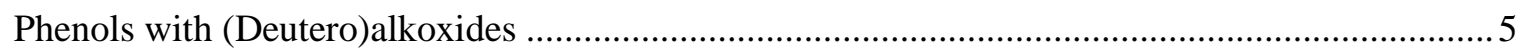

Table S3. Reaction Condition Optimizations for N-Alkylation of Indoles with Alcohols.............. 19

General Procedure and Spectroscopic Data for N-Alkylation of Indoles and Heterocyclic

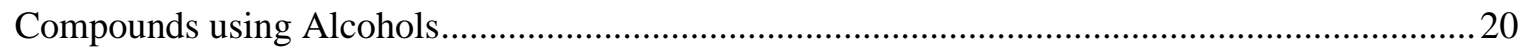

General Procedure for One-pot Reaction of N-Alkylation of Indoles........................................... 31

Synthesis of 8 (Pramoxine) and 8-d (Deuterated Pramoxine) ....................................................... 32

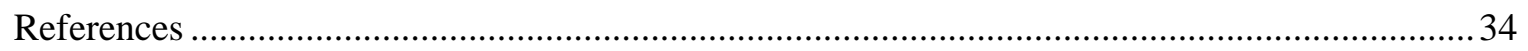

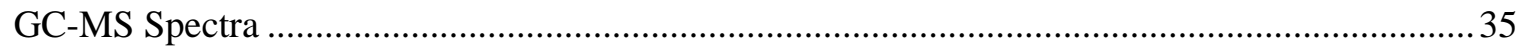

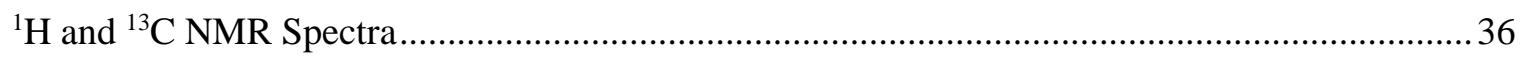




\section{General Information and Materials}

Unless otherwise stated, all reactions were carried out in oven-dried Schlenk glassware under an atmosphere of dry argon or in a glovebox. Reactions were monitored by thin-layer chromatography (TLC). TLC was performed using Huanghai $8 \pm 0.2 \mu \mathrm{m}$ precoated glass plates $(0.25 \mathrm{~mm})$ and visualized by $\mathrm{UV}$ fluorescence quenching, $\mathrm{KMnO}_{4}$, $p$-anisaldehyde, or phosphomolybdic acid staining. Huanghai silica gel (particle size 300-400 or 200-300 mesh) was used for silica gel chromatography. ${ }^{1} \mathrm{H}$ NMR spectra were recorded at room temperature on a Bruker ADVANCE III $400 \mathrm{MHz}$ spectrometer or Oxford NMR AS600 $600 \mathrm{MHz}$ spectrometer and were reported relative to $\mathrm{CDCl}_{3}(\delta 7.26 \mathrm{ppm}) .{ }^{13} \mathrm{C} \mathrm{NMR}$ spectra were recorded on a Bruker ADVANCE III $400 \mathrm{MHz}$ spectrometer and were reported relative to $\mathrm{CDCl}_{3}(\delta 77.16 \mathrm{ppm})$. Data for ${ }^{1} \mathrm{H}$ NMR were reported as chemical shift $(\delta \mathrm{ppm})$ (multiplicity, coupling constant $(\mathrm{Hz})$, integration) using standard abbreviations for multiplicities: $\mathrm{s}=$ singlet, $\mathrm{d}=$ doublet $\mathrm{t}=$ triplet, $\mathrm{q}=$ quartet, quint $=$ quintet, $\mathrm{m}=$ multiplet, and $\mathrm{br}=$ broad signal. Data for ${ }^{13} \mathrm{C}$ NMR were reported in terms of chemical shifts ( $\left.\delta \mathrm{ppm}\right)$. GC-MS analyses were carried out on an Agilent 7890B gas chromatograph equipped with a HP-5 (5\%-phenyl)-methylpolysiloxane capillary column (Agilent) and an Agilent 5977A quadrupole mass-selective detector (MSD) in an electron ionization (EI) mode. High resolution mass spectra (HRMS) were obtained by use of Thermo Fisher Scientific LTQ FTICR-MS spectrometer or Bruker Compact TOF mass spectrometer in an electrospray ionization mode (ESI+) or an atmospheric pressure chemical ionization (APCI+).

Unless otherwise noted, all reagents were purchased commercially and used without further purification. Petroleum ether $\left(60-90{ }^{\circ} \mathrm{C}\right)$ was used as eluent for silica gel chromatography. Dry solvents were purchased commercially or were dried by passage through an activated alumina column under argon. 
Scheme S1. Debrominative Deuteration vs Detosylative Methylation.

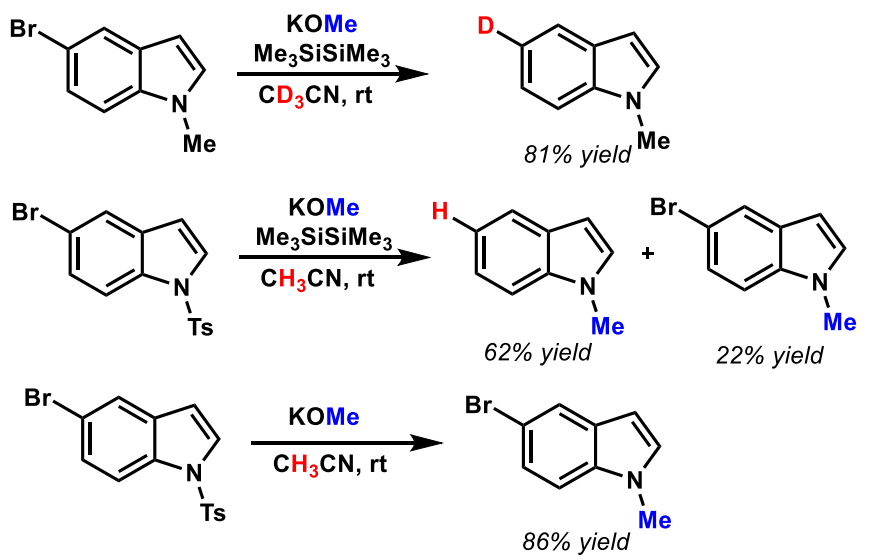

Table S1. Reaction Condition Optimizations for N-Methylation of $\mathrm{N}$ -

Tosylindole.

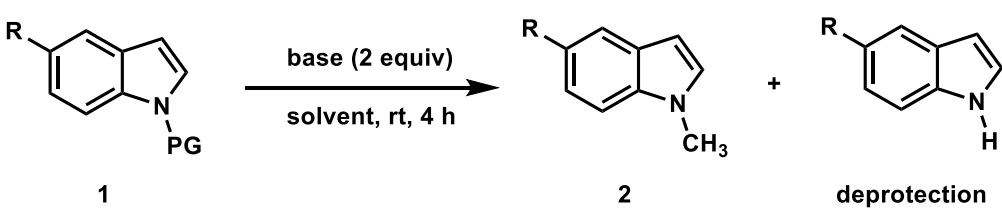

\begin{tabular}{|c|c|c|c|c|c|}
\hline entry $^{a}$ & $\mathrm{R} ; \mathrm{PG}$ & solvent & base & 2 (yield) & deprotection (yield) \\
\hline 1 & $\mathrm{R}=\mathrm{H} ; \mathrm{PG}=\mathrm{Ts}$ & $\mathrm{CH}_{3} \mathrm{CN}$ & $\mathrm{KOCH}_{3}$ & $\mathbf{2 a}, 95 \%^{b}$ & N.D. \\
\hline 2 & $\mathrm{R}=\mathrm{H} ; \mathrm{PG}=\mathrm{Ts}$ & THF & $\mathrm{KOCH}_{3}$ & $\mathbf{2 a}, 95 \%^{b}$ & N.D. \\
\hline 3 & $\mathrm{R}=\mathrm{H} ; \mathrm{PG}=\mathrm{Ts}$ & toluene & $\mathrm{KOCH}_{3}$ & $\mathbf{2 a}, 94 \%^{b}$ & N.D. \\
\hline 4 & $\mathrm{R}=\mathrm{H} ; \mathrm{PG}=\mathrm{Ts}$ & DMF & $\mathrm{KOCH}_{3}$ & $2 \mathbf{a}, 81 \%^{b}$ & N.D. \\
\hline 5 & $\mathrm{R}=\mathrm{H} ; \mathrm{PG}=\mathrm{Ts}$ & DCM & $\mathrm{KOCH}_{3}$ & $\mathbf{2 a}, 72 \%^{b}$ & N.D. \\
\hline 6 & $\mathrm{R}=\mathrm{H} ; \mathrm{PG}=\mathrm{Ts}$ & $\mathrm{CH}_{3} \mathrm{CN}$ & $\mathrm{LiOCH}_{3}$ & 2a, N.D. & N.D. \\
\hline 7 & $\mathrm{R}=\mathrm{H} ; \mathrm{PG}=\mathrm{Ts}$ & $\mathrm{CH}_{3} \mathrm{CN}$ & $\mathrm{NaOCH}_{3}$ & $\mathbf{2 a}, 84 \%^{c}$ & N.D. \\
\hline 8 & $\mathrm{R}=\mathrm{H} ; \mathrm{PG}=\mathrm{Ts}$ & $\mathrm{CH}_{3} \mathrm{CN}$ & $\mathrm{KOCH}_{3}$ & $\mathbf{2 a}, 92 \%^{c}$ & N.D. \\
\hline 9 & $\mathrm{R}=\mathrm{H} ; \mathrm{PG}=\mathrm{PhSO}_{2}$ & $\mathrm{CH}_{3} \mathrm{CN}$ & $\mathrm{KOCH}_{3}$ & $\mathbf{2 a}, 96 \%^{c}$ & N.D. \\
\hline 10 & $\mathrm{R}=\mathrm{H} ; \mathrm{PG}=\mathrm{Tf}$ & $\mathrm{CH}_{3} \mathrm{CN}$ & $\mathrm{KOCH}_{3}$ & 2a, N.D. & $77 \%^{c}$ \\
\hline 11 & $\mathrm{R}=\mathrm{Br} ; \mathrm{PG}=\mathrm{Ac}$ & $\mathrm{CH}_{3} \mathrm{CN}$ & $\mathrm{KOCH}_{3}$ & 2d, N.D. & $20 \%^{c}\left(68 \%^{d}\right)$ \\
\hline
\end{tabular}


${ }^{a}$ Reactions were conducted with $0.2 \mathrm{mmol} \mathbf{1}, 0.4 \mathrm{mmol}$ base in $0.7 \mathrm{~mL}$ solvent. ${ }^{b}$ Yield was determined by GC using dodecane as an internal standard. ${ }^{c}$ Isolated yield. ${ }^{d}$ Recovered starting material. N.D. $=$ not detected.

General Procedure for Condition Optimizations (Table S1): To a screw-capped vial equipped with a magnetic stirring bar were added 1-tosyl- $1 H$-indole 1a (54.2 mg, 0.2 $\mathrm{mmol}), \mathrm{KOCH}_{3}(28.0 \mathrm{mg}, 0.4 \mathrm{mmol})$ and $0.7 \mathrm{~mL}$ solvent. The vial was sealed and stirred until the completion of the reaction as monitored by TLC. Then the vial was removed from the glove box. $\mathrm{H}_{2} \mathrm{O}(10 \mathrm{~mL})$ was added to quench the reaction and the mixture was extracted with DCM $(5 \mathrm{~mL} \times 3)$. The organic layers were washed with brine and dried over anhydrous $\mathrm{Na}_{2} \mathrm{SO}_{4}$, then filtered and evaporated under reduced pressure. The desired product was purified by silica gel chromatography.

\section{Table S2. Reaction Condition Optimizations for O-Methylation of $O$ -}

\section{Tosylphenol.}

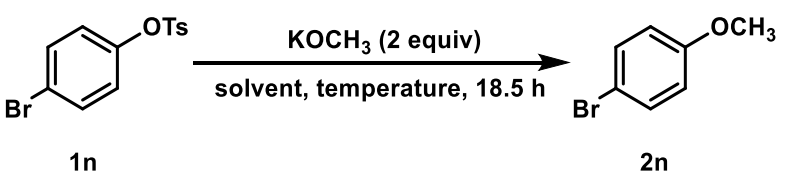

\begin{tabular}{cccc}
\hline entry $^{a}$ & solvent & temperature & yield \\
1 & $\mathrm{DCM}$ & $\mathrm{rt}$ & $39 \%$ \\
2 & $\mathrm{THF}$ & $\mathrm{rt}$ & $30 \%$ \\
3 & $\mathrm{CH}_{3} \mathrm{CN}$ & $\mathrm{rt}$ & $41 \%$ \\
4 & $\mathrm{CH}_{3} \mathrm{CN}$ & $45^{\circ} \mathrm{C}$ & $82 \%$ \\
\hline
\end{tabular}

${ }^{a}$ Reactions were conducted with $0.2 \mathrm{mmol}$ 4-bromophenyl 4-methylbenzenesulfonate (1n), $0.4 \mathrm{mmol}$ $\mathrm{KOCH}_{3}$ in $2 \mathrm{~mL}$ solvent for $18.5 \mathrm{~h} .{ }^{b}$ Isolated yield.

General Procedure for Condition Optimizations (Table S2): To a screw-capped vial equipped with a magnetic stirring bar were added 4-bromophenyl 4methylbenzenesulfonate 1n (65.4 mg, $0.2 \mathrm{mmol}), \mathrm{KOCH}_{3}(28.0 \mathrm{mg}, 0.4 \mathrm{mmol})$ and $2 \mathrm{~mL}$ solvent. The vial was sealed and stirred at indicated temperature for $18.5 \mathrm{~h}$. Then the vial 
was removed from the glove box. The reaction mixture was filtered through a short pad of Celite, and washed by $\mathrm{Et}_{2} \mathrm{O}$ (diethyl ether) (about $4-5 \mathrm{~mL}$ ). The filtrate was concentrated under reduced pressure. The desired product was purified by silica gel chromatography.

General Procedure and Spectroscopic Data for Detosylation/(Deutero)alkylation of Indoles and Phenols with (Deutero)alkoxides

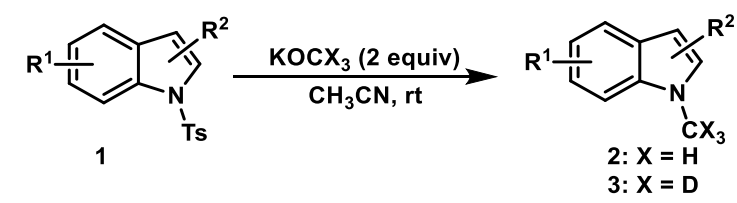

General procedure A: To a dry schlenk tube equipped with a magnetic stirring bar were added tosylated heterocycles 1 (1.0 equiv), $\mathrm{KOCH}_{3}$ or $\mathrm{KOCD}_{3}$ (2.0 equiv) and $\mathrm{CH}_{3} \mathrm{CN}$ under an atmosphere of dry argon. The mixture was stirred at room temperature until the substrate 1 was completely consumed as monitored by TLC. The reaction was quenched with $\mathrm{H}_{2} \mathrm{O}(10 \mathrm{~mL})$ and extracted with $\mathrm{DCM}(10 \mathrm{~mL} \times 3)$. The combined organic layers were washed with brine and dried over anhydrous $\mathrm{Na}_{2} \mathrm{SO}_{4}$, filtered and concentrated under reduced pressure. Then the desired product was purified by silica gel chromatography.

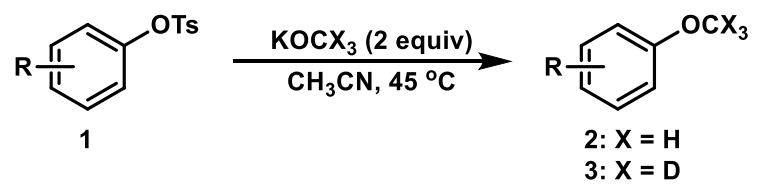

General procedure $B$ : To a dry schlenk tube equipped with a magnetic stirring bar were added $p$-toluenesulfonylphenolic esters 1 (1.0 equiv), $\mathrm{KOCH}_{3}$ or $\mathrm{KOCD}_{3}$ (2.0 equiv), and $\mathrm{CH}_{3} \mathrm{CN}$ under an atmosphere of dry argon. The mixture was stirred at $45{ }^{\circ} \mathrm{C}$ until the substrate 1 was completely consumed as monitored by TLC. Then, the reaction mixture was filtered through a short pad of Celite, and washed with $\mathrm{Et}_{2} \mathrm{O}$. The filtrate was concentrated under reduced pressure at $0{ }^{\circ} \mathrm{C}$. The crude mixture was purified by silica gel chromatography to give the desired product. 


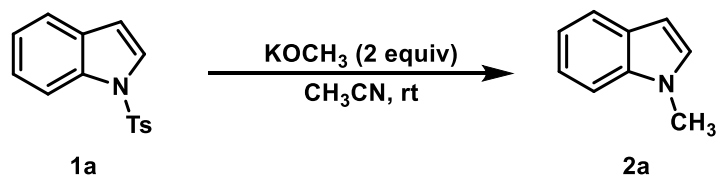

1-Methyl-1H-indole (2a): The general procedure A was followed. The reaction was performed with 1-tosyl-1 $H$-indole $1 \mathrm{a}(54.3 \mathrm{mg}, 0.2 \mathrm{mmol}), \mathrm{KOCH}_{3}(28.0 \mathrm{mg}, 0.4 \mathrm{mmol})$ in $0.7 \mathrm{~mL} \mathrm{CH}{ }_{3} \mathrm{CN}$. The desired product 2a $(24.0 \mathrm{mg}$, $92 \%$ yield) was obtained after purification by silica gel chromatography (1\% EA in PE). ${ }^{1} \mathrm{H}$ NMR $\left(600 \mathrm{MHz}, \mathrm{CDCl}_{3}\right) \delta$ $7.70-7.62(\mathrm{~m}, 1 \mathrm{H}), 7.35(\mathrm{~d}, J=8.2 \mathrm{~Hz}, 1 \mathrm{H}), 7.31-7.20(\mathrm{~m}, 1 \mathrm{H}), 7.18-7.09(\mathrm{~m}, 1 \mathrm{H})$, $7.07(\mathrm{~d}, J=2.8 \mathrm{~Hz}, 1 \mathrm{H}), 6.51(\mathrm{~d}, J=3.1 \mathrm{~Hz}, 1 \mathrm{H}), 3.81(\mathrm{~s}, 3 \mathrm{H})$. These spectroscopic data were consistent with those reported in the literature. ${ }^{1}$

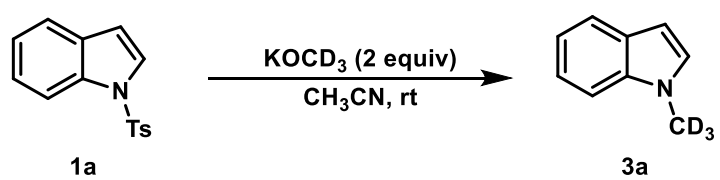

1-Trideuteromethyl-1H-indole (3a): The general procedure A was followed. The reaction was performed with 1-tosyl-1H-indole $1 \mathrm{a}(135.6 \mathrm{mg}, 0.5 \mathrm{mmol}), \mathrm{KOCD}_{3}(73.2 \mathrm{mg}, 1.0$ $\mathrm{mmol}$ ) in $2 \mathrm{~mL} \mathrm{CH}{ }_{3} \mathrm{CN}$. The desired product 3a (59.1 mg, $88 \%$ yield) was obtained after purification by silica gel chromatography (1\% EA in PE). ${ }^{1} \mathrm{H}$ NMR $\left(400 \mathrm{MHz}, \mathrm{CDCl}_{3}\right) \delta$ $7.75(\mathrm{~d}, J=7.9 \mathrm{~Hz}, 1 \mathrm{H}), 7.42(\mathrm{~d}, J=8.4 \mathrm{~Hz}, 1 \mathrm{H}), 7.38-7.29(\mathrm{~m}, 1 \mathrm{H}), 7.27-7.18(\mathrm{~m}, 1 \mathrm{H})$, $7.12(\mathrm{~d}, J=2.9 \mathrm{~Hz}, 1 \mathrm{H}), 6.60(\mathrm{~d}, J=3.0 \mathrm{~Hz}, 1 \mathrm{H}) ;{ }^{13} \mathrm{C} \mathrm{NMR}\left(100 \mathrm{MHz}, \mathrm{CDCl}_{3}\right) \delta 136.7$, 128.8, 128.5, 121.5, 120.9, 119.3, 109.3, 100.9, 32.2 (m); GCMS: calc'd for $\mathrm{C}_{9} \mathrm{H}_{6} \mathrm{D}_{3} \mathrm{~N}[\mathrm{M}]^{+}$: 134.09, found: 134.10 .

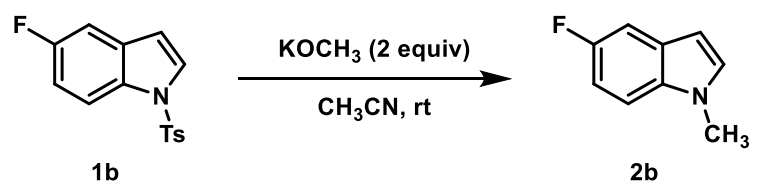

5-Fluoro-1-methyl-1H-indole (2b): The general procedure A was followed. The reaction was performed with 5-fluoro-1-tosyl- $1 H$-indole $\mathbf{1 b}$ (57.9 mg, $0.2 \mathrm{mmol}), \mathrm{KOCH}_{3}(28.0 \mathrm{mg}$, $0.4 \mathrm{mmol})$ in $0.7 \mathrm{~mL} \mathrm{CH}_{3} \mathrm{CN}$. The desired product $\mathbf{2 b}$ ( $28.1 \mathrm{mg}$, 94\% yield) was obtained after purification by silica gel chromatography (1\% EA in PE). ${ }^{1} \mathrm{H} \mathrm{NMR}\left(600 \mathrm{MHz}, \mathrm{CDCl}_{3}\right)$ 
$\delta 7.29-7.26(\mathrm{~m}, 1 \mathrm{H}), 7.23(\mathrm{dd}, J=8.9,4.3 \mathrm{~Hz}, 1 \mathrm{H}), 7.09(\mathrm{~d}, J=2.9 \mathrm{~Hz}, 1 \mathrm{H}), 6.98(\mathrm{td}, J=$ 9.1, $2.5 \mathrm{~Hz}, 1 \mathrm{H}), 6.45(\mathrm{dd}, J=3.2,1.0 \mathrm{~Hz}, 1 \mathrm{H}), 3.79(\mathrm{~s}, 3 \mathrm{H})$. These spectroscopic data were consistent with those reported in the literature. ${ }^{2}$

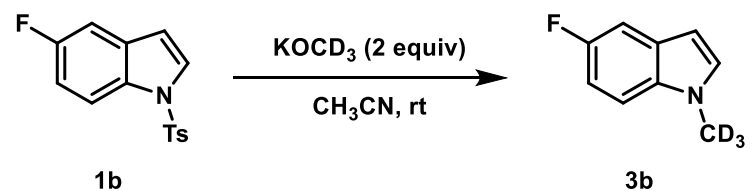

5-Fluoro-1-Trideuteromethyl-1H-indole (3b): The general procedure A was followed. The reaction was performed with 5-fluoro-1-tosyl-1H-indole $\mathbf{1 b}(144.6 \mathrm{mg}, 0.5 \mathrm{mmol})$, $\mathrm{KOCD}_{3}(73.2 \mathrm{mg}, 1.0 \mathrm{mmol})$ in $2 \mathrm{~mL} \mathrm{CH} 3 \mathrm{CN}$. The desired product $3 \mathbf{b}(64.8 \mathrm{mg}, 86 \%$ yield $)$ was obtained after purification by silica gel chromatography (1\% EA in PE). ${ }^{1} \mathrm{H}$ NMR (400 $\left.\mathrm{MHz}, \mathrm{CDCl}_{3}\right) \delta 7.22(\mathrm{dd}, J=9.7,2.5 \mathrm{~Hz}, 1 \mathrm{H}), 7.15(\mathrm{dd}, J=8.8,4.3 \mathrm{~Hz}, 1 \mathrm{H}), 7.01(\mathrm{~d}, J=$ $3.0 \mathrm{~Hz}, 1 \mathrm{H}), 6.91(\mathrm{td}, J=9.1,2.5 \mathrm{~Hz}, 1 \mathrm{H}), 6.38(\mathrm{dd}, J=3.1,0.8 \mathrm{~Hz}, 1 \mathrm{H}) ;{ }^{13} \mathrm{C} \mathrm{NMR}(100$ $\left.\mathrm{MHz}, \mathrm{CDCl}_{3}\right) \delta 157.9(\mathrm{~d}, J=233.4 \mathrm{~Hz}), 133.5,130.5,128.7(\mathrm{~d}, J=10.3 \mathrm{~Hz}), \delta 110.0(\mathrm{~d}, J$ $=12.8 \mathrm{~Hz}), 109.8(\mathrm{~d}, J=3.8 \mathrm{~Hz}), 105.6(\mathrm{~d}, J=23.3 \mathrm{~Hz}), 100.9(\mathrm{~d}, J=4.8 \mathrm{~Hz}), 32.5(\mathrm{~m})$; GCMS: calc'd for $\mathrm{C}_{9} \mathrm{H}_{5} \mathrm{D}_{3} \mathrm{FN}[\mathrm{M}]^{+}:$152.08, found: 152.10 .

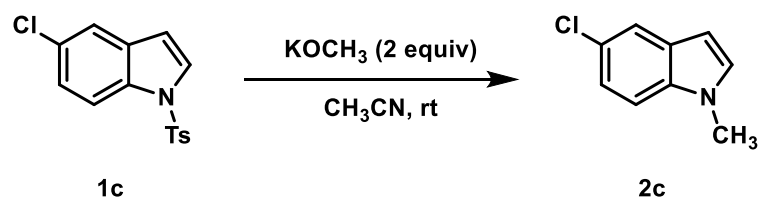

5-Chloro-1-methyl-1H-indole (2c): The general procedure A was followed. The reaction was performed with 5-chloro-1-tosyl-1H-indole $1 \mathrm{c}(61.0 \mathrm{mg}, 0.2 \mathrm{mmol}), \mathrm{KOCH}_{3}(28.0 \mathrm{mg}$, $0.4 \mathrm{mmol})$ in $0.7 \mathrm{~mL} \mathrm{CH}{ }_{3} \mathrm{CN}$. The desired product $2 \mathrm{c}$ (29.6 $\mathrm{mg}$, $89 \%$ yield) was obtained after purification by silica gel chromatography (1\% EA in PE). ${ }^{1} \mathrm{H} \mathrm{NMR}\left(600 \mathrm{MHz}, \mathrm{CDCl}_{3}\right)$ $\delta 7.59(\mathrm{~d}, J=2.1 \mathrm{~Hz}, 1 \mathrm{H}), 7.23(\mathrm{~d}, J=8.7 \mathrm{~Hz}, 1 \mathrm{H}), 7.17(\mathrm{dd}, J=8.7,2.1 \mathrm{~Hz}, 1 \mathrm{H}), 7.07(\mathrm{~d}$, $J=3.0 \mathrm{~Hz}, 1 \mathrm{H}), 6.42(\mathrm{~d}, J=3.0 \mathrm{~Hz}, 1 \mathrm{H}), 3.78(\mathrm{~s}, 3 \mathrm{H})$. These spectroscopic data were consistent with those reported in the literature. ${ }^{2}$

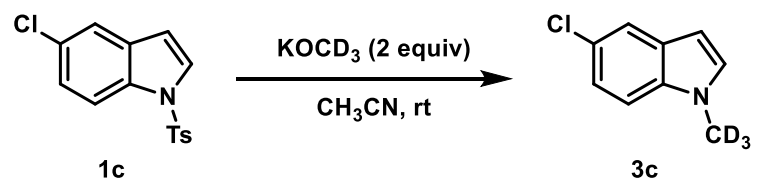


5-Chloro-1-trideuteromethyl-1H-indole (3c): The general procedure A was followed. The reaction was performed with 5-chloro-1-tosyl-1H-indole 1c (152.9 mg, $0.5 \mathrm{mmol})$, $\mathrm{KOCD}_{3}(73.2 \mathrm{mg}, 1.0 \mathrm{mmol})$ in $2 \mathrm{~mL} \mathrm{CH}_{3} \mathrm{CN}$. The desired product $3 \mathbf{c}(72.9 \mathrm{mg}, 86 \%$ yield $)$ was obtained after purification by silica gel chromatography (1\% EA in PE). ${ }^{1} \mathrm{H}$ NMR (400 $\left.\mathrm{MHz}, \mathrm{CDCl}_{3}\right) \delta 7.62(\mathrm{~d}, J=2.1 \mathrm{~Hz}, 1 \mathrm{H}), 7.24(\mathrm{~d}, J=8.6 \mathrm{~Hz}, 1 \mathrm{H}), 7.20(\mathrm{dd}, J=8.7,1.9 \mathrm{~Hz}$, 1H). $7.08(\mathrm{~d}, J=3.1 \mathrm{~Hz}, 1 \mathrm{H}), 6.45(\mathrm{~d}, J=3.0 \mathrm{~Hz}, 1 \mathrm{H}) ;{ }^{13} \mathrm{C} \mathrm{NMR}\left(100 \mathrm{MHz}, \mathrm{CDCl}_{3}\right) \delta$ 135.2, 130.2, 129.5, 125.1, 121.8, 120.2, 110.3, 100.6, 32.4 (m); GCMS: calc'd for $\mathrm{C}_{9} \mathrm{H}_{5} \mathrm{D}_{3} \mathrm{ClN}[\mathrm{M}]^{+}:$168.05, found: 168.05 .

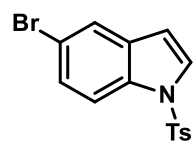

$1 d$

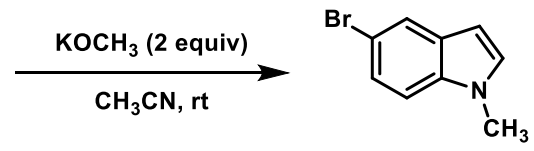

2d

5-Bromo-1-methyl-1H-indole (2d): The general procedure A was followed. The reaction was performed with 5-bromo-1-tosyl-1H-indole $1 \mathbf{d}(70.1 \mathrm{mg}, 0.2 \mathrm{mmol}), \mathrm{KOCH}_{3}(28.0 \mathrm{mg}$, $0.4 \mathrm{mmol})$ in $0.7 \mathrm{~mL} \mathrm{CH}_{3} \mathrm{CN}$. The desired product $\mathbf{2 d}$ (36.3 $\mathrm{mg}, 86 \%$ yield) was obtained after purification by silica gel chromatography (1\% EA in PE). ${ }^{1} \mathrm{H} \mathrm{NMR}\left(600 \mathrm{MHz}, \mathrm{CDCl}_{3}\right)$ $\delta 7.75(\mathrm{~d}, J=1.9 \mathrm{~Hz}, 1 \mathrm{H}), 7.30(\mathrm{dd}, J=8.7,1.9 \mathrm{~Hz}, 1 \mathrm{H}), 7.19(\mathrm{~d}, J=8.7 \mathrm{~Hz}, 1 \mathrm{H}), 7.05(\mathrm{~d}$, $J=3.1 \mathrm{~Hz}, 1 \mathrm{H}), 6.42(\mathrm{~d}, J=3.2 \mathrm{~Hz}, 1 \mathrm{H}), 3.78(\mathrm{~s}, 3 \mathrm{H})$. These spectroscopic data were consistent with those reported in the literature. ${ }^{2}$

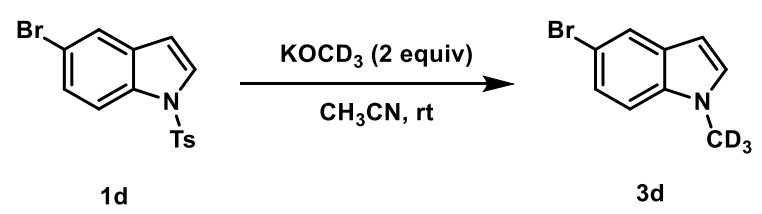

5-Bromo-1-trideuteromethyl-1H-indole (3d): The general procedure A was followed. The reaction was performed with 5-bromo-1-tosyl-1H-indole $1 \mathbf{d d}(175.1 \mathrm{mg}, 0.5 \mathrm{mmol})$, $\mathrm{KOCD}_{3}(73.2 \mathrm{mg}, 1.0 \mathrm{mmol})$ in $2 \mathrm{~mL} \mathrm{CH}_{3} \mathrm{CN}$. The desired product $3 \mathbf{d}$ (96.4 mg, $91 \%$ yield) was obtained after purification by silica gel chromatography (1\% EA in PE). ${ }^{1} \mathrm{H}$ NMR (400 $\left.\mathrm{MHz}, \mathrm{CDCl}_{3}\right) \delta 7.76(\mathrm{~d}, J=1.8 \mathrm{~Hz}, 1 \mathrm{H}), 7.31(\mathrm{dd}, J=8.7,1.9 \mathrm{~Hz}, 1 \mathrm{H}), 7.19(\mathrm{~d}, J=8.6 \mathrm{~Hz}$, $1 \mathrm{H}), 7.05(\mathrm{~d}, J=3.1 \mathrm{~Hz}, 1 \mathrm{H}), 6.44(\mathrm{dd}, J=3.2,0.9 \mathrm{~Hz}, 1 \mathrm{H}) ;{ }^{13} \mathrm{C} \mathrm{NMR}\left(100 \mathrm{MHz}, \mathrm{CDCl}_{3}\right)$ 
$\delta$ 135.4, 130.2, 130.0, 124.3, 123.3, 112.7, 110.8, 100.6, $32.4(\mathrm{~m})$; GCMS: calc'd for $\mathrm{C}_{9} \mathrm{H}_{5} \mathrm{D}_{3} \mathrm{BrN}[\mathrm{M}]^{+}:$212.00, found: 211.95 .

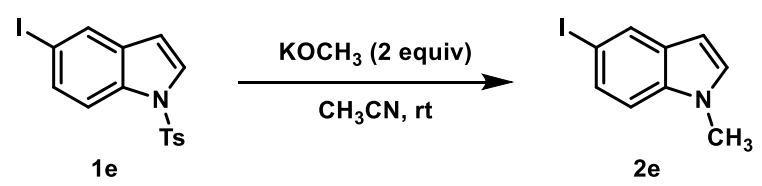

5-Iodo-1-methyl-1H-indole (2e): The general procedure A was followed. The reaction was performed with 5-iodo-1-tosyl- $1 \mathrm{H}$-indole $1 \mathrm{e}(79.4 \mathrm{mg}, 0.2 \mathrm{mmol}), \mathrm{KOCH}_{3}(28.0 \mathrm{mg}$, $0.4 \mathrm{mmol})$ in $0.7 \mathrm{~mL} \mathrm{CH} 3 \mathrm{CN}$. The desired product $2 \mathrm{e}$ (42.3 $\mathrm{mg}$, 82\% yield) was obtained after purification by silica gel chromatography (1\% EA in PE). ${ }^{1} \mathrm{H} \mathrm{NMR}\left(600 \mathrm{MHz}, \mathrm{CDCl}_{3}\right)$ $\delta 7.96(\mathrm{~d}, J=1.9 \mathrm{~Hz}, 1 \mathrm{H}), 7.46(\mathrm{dd}, J=8.6,1.8 \mathrm{~Hz}, 1 \mathrm{H}), 7.10(\mathrm{~d}, J=8.5 \mathrm{~Hz}, 1 \mathrm{H}), 7.03-$ 6.97(m, 1H), $6.43-6.38(\mathrm{~m}, 1 \mathrm{H}), 3.77(\mathrm{~s}, 3 \mathrm{H})$. These spectroscopic data were consistent with those reported in the literature. ${ }^{2}$

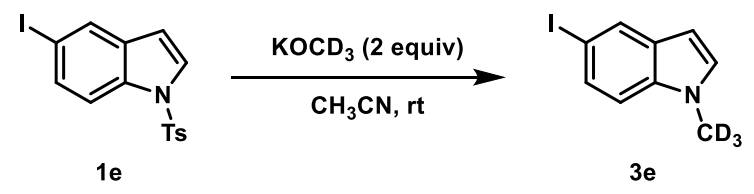

5-Iodo-1-trideuteromethyl-1H-indole (3e): The general procedure A was followed. The reaction was performed with 5-iodo-1-tosyl- $1 H$-indole 1 e $(198.6 \mathrm{mg}, 0.5 \mathrm{mmol}), \mathrm{KOCD}_{3}$ (73.2 $\mathrm{mg}, 1.0 \mathrm{mmol})$ in $2 \mathrm{~mL} \mathrm{CH} 3 \mathrm{CN}$. The desired product $3 \mathbf{e}(121.7 \mathrm{mg}$, 94\% yield) was obtained after purification by silica gel chromatography (1\% EA in PE). ${ }^{1} \mathrm{H}$ NMR (400 $\left.\mathrm{MHz}, \mathrm{CDCl}_{3}\right) \delta 7.97(\mathrm{~d}, J=1.6 \mathrm{~Hz}, 1 \mathrm{H}), 7.47(\mathrm{dd}, J=8.6,1.7 \mathrm{~Hz}, 1 \mathrm{H}), 7.10(\mathrm{~d}, J=8.5 \mathrm{~Hz}$, $1 \mathrm{H}), 7.01(\mathrm{~d}, J=8.6 \mathrm{~Hz}, 1 \mathrm{H}), 6.42(\mathrm{dd}, J=3.1,0.9 \mathrm{~Hz}, 1 \mathrm{H}) ;{ }^{13} \mathrm{C} \mathrm{NMR}\left(100 \mathrm{MHz}, \mathrm{CDCl}_{3}\right)$ $\delta$ 135.8, 131.0, 129.8, 129.7, 129.6, 111.3, 100.3, 82.9, 32.3 (m); GCMS: calc'd for $\mathrm{C}_{9} \mathrm{H}_{5} \mathrm{D}_{3} \mathrm{IN}[\mathrm{M}]^{+}:$259.99, found: 259.95 .

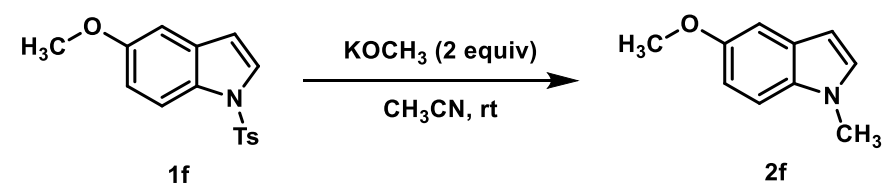

5-Methoxy-1-methyl-1H-indole (2f): The general procedure A was followed. The reaction was performed with 5-methoxy-1-tosyl- $1 H$-indole $\mathbf{1 f}(150.7 \mathrm{mg}, 0.5 \mathrm{mmol}), \mathrm{KOCH}_{3}(70.0$ 
$\mathrm{mg}, 1.0 \mathrm{mmol})$ in $1 \mathrm{~mL} \mathrm{CH} 3 \mathrm{CN}$. The desired product $\mathbf{2 f}(73.7 \mathrm{mg}, 91 \%$ yield $)$ was obtained after purification by silica gel chromatography (1\% EA in PE). ${ }^{1} \mathrm{H} \mathrm{NMR}\left(600 \mathrm{MHz}, \mathrm{CDCl}_{3}\right)$ $\delta 7.22(\mathrm{~d}, J=8.8 \mathrm{~Hz}, 1 \mathrm{H}), 7.09(\mathrm{~d}, J=2.5 \mathrm{~Hz}, 1 \mathrm{H}), 7.02(\mathrm{~s}, 1 \mathrm{H}), 6.89(\mathrm{dd}, J=8.9,2.5 \mathrm{~Hz}$, $1 \mathrm{H}), 6.40(\mathrm{~s}, 1 \mathrm{H}), 3.85(\mathrm{~s}, 3 \mathrm{H}), 3.77(\mathrm{~s}, 3 \mathrm{H})$. These spectroscopic data were consistent with those reported in the literature. ${ }^{2}$

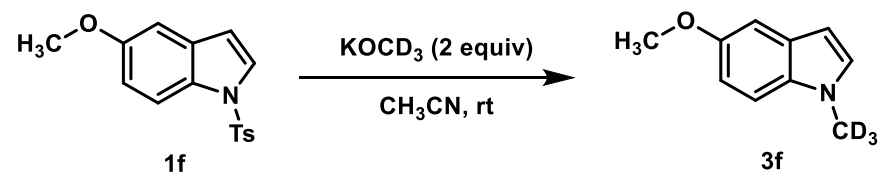

5-Methoxy-1-trideuteromethyl-1H-indole (3f): The general procedure A was followed. The reaction was performed with 5-methoxy-1-tosyl- $1 H$-indole $\mathbf{1 f}(150.7 \mathrm{mg}, 0.5 \mathrm{mmol})$, $\mathrm{KOCD}_{3}(73.2 \mathrm{mg}, 1.0 \mathrm{mmol})$ in $1 \mathrm{~mL} \mathrm{CH} 3 \mathrm{CN}$. The desired product $3 \mathbf{f}(64.6 \mathrm{mg}, 79 \%$ yield $)$ was obtained after purification by silica gel chromatography (1\% EA in PE). ${ }^{1} \mathrm{H}$ NMR (400 $\left.\mathrm{MHz}, \mathrm{CDCl}_{3}\right) \delta 7.24(\mathrm{~d}, J=8.9 \mathrm{~Hz}, 1 \mathrm{H}), 7.13(\mathrm{~d}, J=2.4 \mathrm{~Hz}, 1 \mathrm{H}), 7.04(\mathrm{~d}, J=3.0 \mathrm{~Hz}, 1 \mathrm{H})$, $6.93(\mathrm{dd}, J=8.8,2.4 \mathrm{~Hz}, 1 \mathrm{H}), 6.44(\mathrm{dd}, J=3.0,0.8 \mathrm{~Hz}, 1 \mathrm{H}), 3.88(\mathrm{~s}, 3 \mathrm{H}) ;{ }^{13} \mathrm{C}$ NMR $(100$ $\left.\mathrm{MHz}, \mathrm{CDCl}_{3}\right) \delta 154.0,132.1,129.3,128.8,111.9,110.0,102.5,100.4,56.0,32.3(\mathrm{~m})$; GCMS: calc'd for $\mathrm{C}_{10} \mathrm{H}_{8} \mathrm{D}_{3} \mathrm{NO}[\mathrm{M}]^{+}:$: 164.10, found: 164.10 .
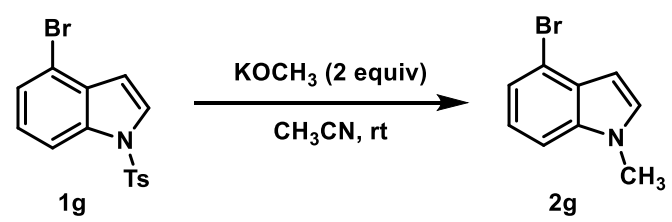

4-Bromo-1-methyl-1H-indole (2g): The general procedure A was followed. The reaction was performed with 4-bromo-1-tosyl- $1 \mathrm{H}$-indole $1 \mathrm{~g}$ (70.1 mg, $0.2 \mathrm{mmol}), \mathrm{KOCH}_{3}(28.0 \mathrm{mg}$, $0.4 \mathrm{mmol}$ ) in $0.7 \mathrm{~mL} \mathrm{CH} 3 \mathrm{CN}$. The desired product $2 \mathrm{~g}$ (37.4 $\mathrm{mg}, 89 \%$ yield) was obtained after purification by silica gel chromatography (1\% EA in PE). ${ }^{1} \mathrm{H} \mathrm{NMR}\left(600 \mathrm{MHz}, \mathrm{CDCl}_{3}\right)$ $\delta 7.35-7.27(\mathrm{~m}, 2 \mathrm{H}), 7.13-7.03(\mathrm{~m}, 2 \mathrm{H}), 6.54(\mathrm{~d}, J=3.1 \mathrm{~Hz}, 1 \mathrm{H}), 3.78(\mathrm{~s}, 3 \mathrm{H})$. These spectroscopic data were consistent with those reported in the literature. ${ }^{3}$<smiles>Cn1ccc2c(Br)cccc21</smiles>

$1 \mathrm{~g}$

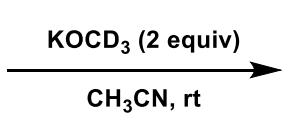

SI-10

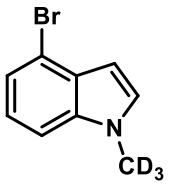

3g 
4-Bromo-1-trideuteromethyl-1H-indole (3g): The general procedure A was followed. The reaction was performed with 4-bromo-1-tosyl-1H-indole $1 \mathrm{~g}$ (70.0 mg, $0.2 \mathrm{mmol})$, $\mathrm{KOCD}_{3}(29.3 \mathrm{mg}, 0.4 \mathrm{mmol})$ in $1 \mathrm{~mL} \mathrm{CH}_{3} \mathrm{CN}$. The desired product $\mathbf{3 g}$ ( $75.5 \mathrm{mg}, 71 \%$ yield) was obtained after purification by silica gel chromatography (1\% EA in PE). ${ }^{1} \mathrm{H}$ NMR (400 $\left.\mathrm{MHz}, \mathrm{CDCl}_{3}\right) \delta 7.31-7.28(\mathrm{~m}, 1 \mathrm{H}), 7.28-7.26(\mathrm{~m}, 1 \mathrm{H}), 7.11(\mathrm{~d}, J=3.3 \mathrm{~Hz}, 1 \mathrm{H}), 7.07(\mathrm{~d}$, $J=8.3 \mathrm{~Hz}, 1 \mathrm{H}), 6.53(\mathrm{dd}, J=3.1,0.9 \mathrm{~Hz}, 1 \mathrm{H}) ;{ }^{13} \mathrm{C} \mathrm{NMR}\left(100 \mathrm{MHz}, \mathrm{CDCl}_{3}\right) \delta 137.1,129.4$, 129.2, 122.5, 122.3, 114.9, 108.5, 101.4, 32.6 (m); GCMS: calc'd for $\mathrm{C}_{9} \mathrm{H}_{5} \mathrm{D}_{3} \mathrm{NBr}[\mathrm{M}]^{+}$: 212.00, found: 211.95 .

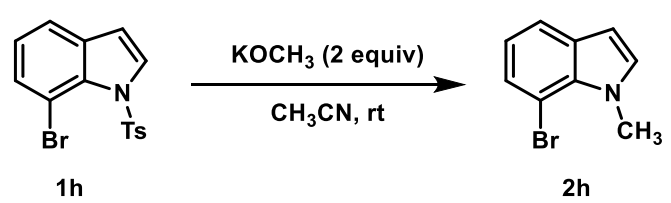

7-Bromo-1-methyl-1H-indole (2h): The general procedure A was followed. The reaction was performed with 7-bromo-1-tosyl-1H-indole $\mathbf{1 h}(70.1 \mathrm{mg}, 0.2 \mathrm{mmol}), \mathrm{KOCH}_{3}(28.0 \mathrm{mg}$, $0.4 \mathrm{mmol})$ in $0.7 \mathrm{~mL} \mathrm{CH} 3 \mathrm{CN}$. The desired product $\mathbf{2 h}$ (31.9 $\mathrm{mg}, 76 \%$ yield) was obtained after purification by silica gel chromatography (1\% EA in PE). ${ }^{1} \mathrm{H} \mathrm{NMR}\left(600 \mathrm{MHz}, \mathrm{CDCl}_{3}\right)$ $\delta 7.54(\mathrm{~d}, J=7.8 \mathrm{~Hz}, 1 \mathrm{H}), 7.34(\mathrm{~d}, J=7.6 \mathrm{~Hz}, 1 \mathrm{H}), 6.99(\mathrm{~d}, J=3.0 \mathrm{~Hz}, 1 \mathrm{H}), 6.91(\mathrm{t}, J=$ $7.7 \mathrm{~Hz}, 1 \mathrm{H}), 6.46(\mathrm{dd}, J=3.1,0.9 \mathrm{~Hz}, 1 \mathrm{H}), 4.16(\mathrm{~s}, 3 \mathrm{H})$. These spectroscopic data were consistent with those reported in the literature. ${ }^{2}$

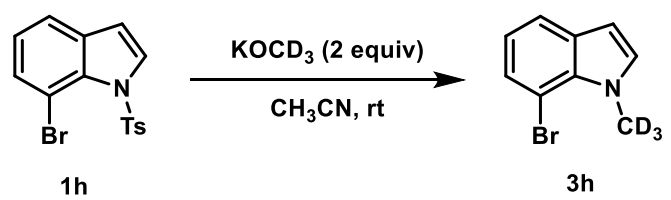

7-Bromo-1-trideuteromethyl- $1 \mathrm{H}$-indole $(3 \mathrm{~h})$ : The general procedure A was followed. The reaction was performed with 7-bromo-1-tosyl-1H-indole $\mathbf{1 h}(175.1 \mathrm{mg}, 0.5 \mathrm{mmol})$, $\mathrm{KOCD}_{3}(73.2 \mathrm{mg}, 1.0 \mathrm{mmol})$ in $2 \mathrm{~mL} \mathrm{CH}_{3} \mathrm{CN}$. The desired product $3 \mathbf{h}$ ( $97.2 \mathrm{mg}, 92 \%$ yield) was obtained after purification by silica gel chromatography (1\% EA in PE). ${ }^{1} \mathrm{H}$ NMR (400 $\left.\mathrm{MHz}, \mathrm{CDCl}_{3}\right) \delta 7.55(\mathrm{~d}, J=7.8 \mathrm{~Hz}, 1 \mathrm{H}), 7.35(\mathrm{~d}, J=7.6 \mathrm{~Hz}, 1 \mathrm{H}), 7.00(\mathrm{~d}, J=3.1 \mathrm{~Hz}, 1 \mathrm{H})$, $6.92(\mathrm{t}, J=7.7 \mathrm{~Hz}, 1 \mathrm{H}), 6.47(\mathrm{~d}, J=3.0 \mathrm{~Hz}, 1 \mathrm{H}) ;{ }^{13} \mathrm{C} \mathrm{NMR}\left(100 \mathrm{MHz}, \mathrm{CDCl}_{3}\right) \delta 133.2$, 
131.84, 131.75, 126.6, 120.6, 120.5, 104.0, 101.3, 36.1 (m); GCMS: calc'd for $\mathrm{C}_{9} \mathrm{H}_{5} \mathrm{D}_{3} \mathrm{NBr}$ $[\mathrm{M}]^{+}:$212.00, found: 211.95 .

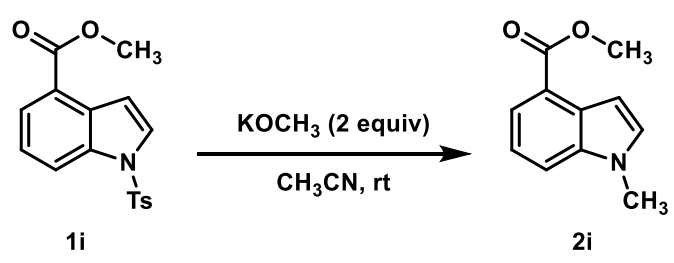

Methyl 1-Methyl-1H-indole-4-carboxylate (2i): The general procedure A was followed. The reaction was performed with methyl 1-tosyl-1H-indole-4-carboxylate $1 \mathbf{i}$ (65.9 mg, 0.2 $\mathrm{mmol}), \mathrm{KOCH}_{3}(28.0 \mathrm{mg}, 0.4 \mathrm{mmol})$ in $0.7 \mathrm{~mL} \mathrm{CH}_{3} \mathrm{CN}$. The desired product $2 \mathbf{i}(24.7 \mathrm{mg}$, $65 \%$ yield) was obtained after purification by silica gel chromatography (10\% EA in PE). ${ }^{1} \mathrm{H}$ NMR (400 MHz, $\left.\mathrm{CDCl}_{3}\right) \delta 7.91(\mathrm{dd}, J=7.5,1.0 \mathrm{~Hz}, 1 \mathrm{H}), 7.53(\mathrm{~d}, J=9.1 \mathrm{~Hz}, 1 \mathrm{H}), 7.26$ $(\mathrm{t}, J=7.8 \mathrm{~Hz}, 1 \mathrm{H}), 7.20(\mathrm{~d}, J=3.1 \mathrm{~Hz}, 1 \mathrm{H}), 7.11(\mathrm{dd}, J=3.1,0.9 \mathrm{~Hz}, 1 \mathrm{H}), 3.98(\mathrm{~s}, 3 \mathrm{H})$, $3.84(\mathrm{~s}, 3 \mathrm{H})$. These spectroscopic data were consistent with those reported in the literature. ${ }^{4}$<smiles>COC(=O)c1ccc2c(ccn2C)c1</smiles>

$1 \mathrm{j}$

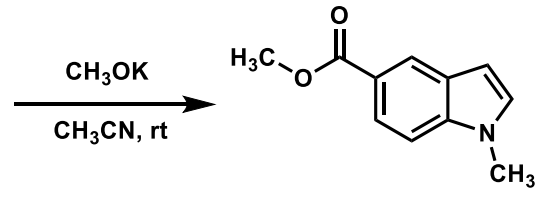

$2 \mathbf{j}$

Methyl 1-Methyl-1H-indole-5-carboxylate (2j): The general procedure A was followed. The reaction was performed with methyl 1-tosyl-1H-indole-5-carboxylate $\mathbf{1 j}$ (65.9 mg, 0.2 $\mathrm{mmol}), \mathrm{KOCH}_{3}\left(28.0 \mathrm{mg}, 0.4 \mathrm{mmol}\right.$ ) in $0.7 \mathrm{~mL} \mathrm{CH}_{3} \mathrm{CN}$. The desired product $\mathbf{2 j}$ (33.3 mg, $90 \%$ yield) was obtained after purification by silica gel chromatography (10\% EA in PE). ${ }^{1} \mathrm{H}$ NMR $\left(400 \mathrm{MHz}, \mathrm{CDCl}_{3}\right) \delta 8.40(\mathrm{~d}, J=1.0 \mathrm{~Hz}, 1 \mathrm{H}), 7.93(\mathrm{dd}, J=8.7,1.6 \mathrm{~Hz}, 1 \mathrm{H}), 7.32$ $(\mathrm{d}, J=8.6 \mathrm{~Hz}, 1 \mathrm{H}), 7.11(\mathrm{~d}, J=3.2 \mathrm{~Hz}, 1 \mathrm{H}), 6.59(\mathrm{dd}, J=3.2,0.9 \mathrm{~Hz}, 1 \mathrm{H}), 3.93(\mathrm{~s}, 3 \mathrm{H})$, $3.82(\mathrm{~s}, 3 \mathrm{H})$. These spectroscopic data were consistent with those reported in the literature. ${ }^{5}$

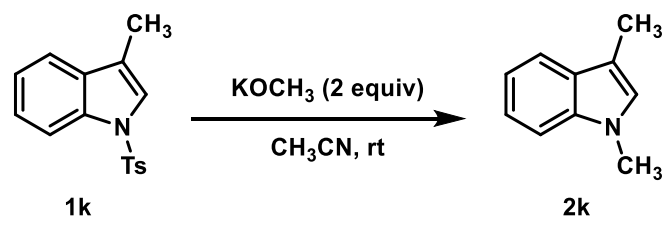


1,3-Dimethyl-1H-indole (2k): The general procedure A was followed. The reaction was performed with 3-methyl-1-tosyl-1H-indole $1 \mathbf{k}(57.1 \mathrm{mg}, 0.2 \mathrm{mmol}), \mathrm{KOCH}_{3}(28.0 \mathrm{mg}$, $0.4 \mathrm{mmol})$ in $0.7 \mathrm{~mL} \mathrm{CH}_{3} \mathrm{CN}$. The desired product $2 \mathbf{k}(25.2 \mathrm{mg}, 87 \%$ yield) was obtained after purification by silica gel chromatography (1\% EA in PE). ${ }^{1} \mathrm{H} \mathrm{NMR}\left(600 \mathrm{MHz}, \mathrm{CDCl}_{3}\right)$ $\delta 7.58(\mathrm{~d}, J=7.9 \mathrm{~Hz}, 1 \mathrm{H}), 7.29(\mathrm{~d}, J=8.1 \mathrm{~Hz}, 1 \mathrm{H}), 7.23(\mathrm{t}, J=7.6 \mathrm{~Hz}, 1 \mathrm{H}), 7.11(\mathrm{t}, J=7.4$ $\mathrm{Hz}, 1 \mathrm{H}), 6.83(\mathrm{~s}, 1 \mathrm{H}), 3.74(\mathrm{~s}, 3 \mathrm{H}), 2.33$ (s, 3H). These spectroscopic data were consistent with those reported in the literature. ${ }^{6}$

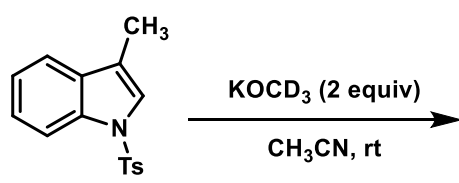

$1 \mathrm{k}$

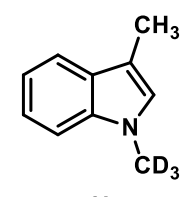

$3 \mathbf{i}$

3-Methyl-1-trideuteromethyl-1H-indole (3i): The general procedure A was followed. The reaction was performed with 3-methyl-1-tosyl- $1 H$-indole $1 \mathbf{k}(142.7 \mathrm{mg}, 0.5 \mathrm{mmol})$, $\mathrm{KOCD}_{3}(73.2 \mathrm{mg}, 1.0 \mathrm{mmol})$ in $2 \mathrm{~mL} \mathrm{CH} 3 \mathrm{CN}$. The desired product $3 \mathbf{i}(71.1 \mathrm{mg}, 97 \%$ yield $)$ was obtained after purification by silica gel chromatography (1\% EA in PE). ${ }^{1} \mathrm{H}$ NMR (400 $\left.\mathrm{MHz}, \mathrm{CDCl}_{3}\right) \delta 7.50(\mathrm{~d}, J=7.2 \mathrm{~Hz}, 1 \mathrm{H}), 7.20(\mathrm{~d}, J=8.3 \mathrm{~Hz}, 1 \mathrm{H}), 7.17-7.10(\mathrm{~m}, 1 \mathrm{H})$, $7.06-7.00(\mathrm{~m}, 1 \mathrm{H}), 6.73(\mathrm{~s}, 1 \mathrm{H}), 2.26(\mathrm{~s}, 3 \mathrm{H}) ;{ }^{13} \mathrm{C} \mathrm{NMR}\left(100 \mathrm{MHz}, \mathrm{CDCl}_{3}\right) \delta 137.1,128.7$, 126.6, 121.5, 119.0, 118.6, 110.1, 109.1, 31.9 (m), 9.7; GCMS: calc'd for $\mathrm{C}_{10} \mathrm{H}_{8} \mathrm{D}_{3} \mathrm{~N}[\mathrm{M}]^{+}$: 148.11, found: 148.10 .

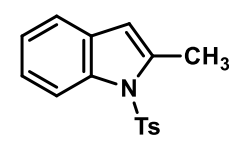

11

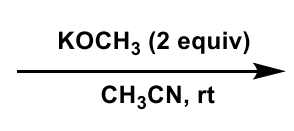

$\mathrm{CH}_{3} \mathrm{CN}, \mathrm{rt}$

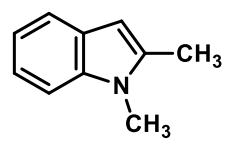

21

1,2-Dimethyl-1H-indole (2l): The general procedure A was followed. The reaction was performed with 2-methyl-1-tosyl-1H-indole 11 (57.1 mg, $0.2 \mathrm{mmol}), \mathrm{KOCH}_{3}(28.0 \mathrm{mg}, 0.4$ $\mathrm{mmol}$ ) in $0.7 \mathrm{~mL} \mathrm{CH}_{3} \mathrm{CN}$. The desired product $2 \mathrm{l}$ (14.7 $\mathrm{mg}, 51 \%$ yield) was obtained after purification by silica gel chromatography (1\% EA in PE). ${ }^{1} \mathrm{H}$ NMR $\left(600 \mathrm{MHz}, \mathrm{CDCl}_{3}\right) \delta$ $7.52(\mathrm{~d}, J=7.7 \mathrm{~Hz}, 1 \mathrm{H}), 7.25(\mathrm{~d}, J=8.0 \mathrm{~Hz}, 1 \mathrm{H}), 7.15(\mathrm{t}, J=7.6 \mathrm{~Hz}, 1 \mathrm{H}), 7.06(\mathrm{t}, J=7.4$ Hz, 1H). 3.66 (s, 3H), 2.43 (s, 3H). These spectroscopic data were consistent with those reported in the literature. ${ }^{7}$ 


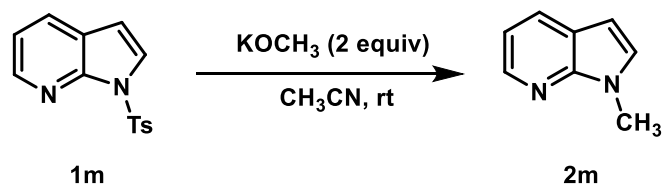

1-Methyl-1H-pyrrolo[2,3-b]pyridine (2m): The general procedure A was followed. The reaction was performed with 1-tosyl-1 $H$-pyrrolo[2,3-b]pyridine $\mathbf{1 m}(54.5 \mathrm{mg}, 0.2 \mathrm{mmol})$, $\mathrm{KOCH}_{3}(28.0 \mathrm{mg}, 0.4 \mathrm{mmol})$ in $0.7 \mathrm{~mL} \mathrm{CH}_{3} \mathrm{CN}$. The desired product $\mathbf{2 m}(22.5 \mathrm{mg}, 85 \%$ yield) was obtained after purification by silica gel chromatography (1\% EA in PE). ${ }^{1} \mathrm{H}$ NMR (600 MHz, $\left.\mathrm{CDCl}_{3}\right) \delta 8.34(\mathrm{dd}, J=4.7,1.6 \mathrm{~Hz}, 1 \mathrm{H}), 7.91(\mathrm{dd}, J=7.8,1.6 \mathrm{~Hz}, 1 \mathrm{H})$, $7.18(\mathrm{~d}, J=3.4 \mathrm{~Hz}, 1 \mathrm{H}), 7.06(\mathrm{dd}, J=7.8,4.7 \mathrm{~Hz}, 1 \mathrm{H}), 6.45(\mathrm{~d}, J=3.4 \mathrm{~Hz}, 1 \mathrm{H}), 3.90(\mathrm{~s}$, $3 \mathrm{H})$. These spectroscopic data were consistent with those reported in the literature. ${ }^{8}$

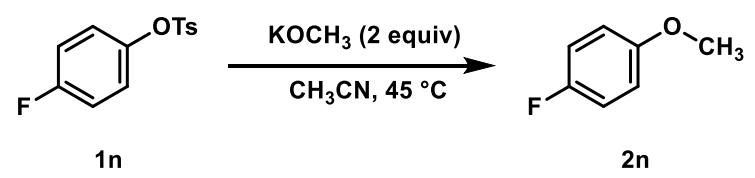

1-Fluoro-4-methoxybenzene (2n): The general procedure B was followed. The reaction was performed with 4-fluorophenyl 4-methylbenzenesulfonate $1 \mathrm{n}(132.8 \mathrm{mg}, 0.5 \mathrm{mmol})$, $\mathrm{KOCH}_{3}(70.5 \mathrm{mg}, 1.0 \mathrm{mmol})$ in $2 \mathrm{~mL} \mathrm{CH}_{3} \mathrm{CN}$ for $18.5 \mathrm{~h}$. The desired product $2 \mathrm{n}(49.0 \mathrm{mg}$, $76 \%$ yield) was obtained after purification by silica gel chromatography (2\% EA in PE). ${ }^{1} \mathrm{H} \mathrm{NMR}\left(400 \mathrm{MHz}, \mathrm{CDCl}_{3}\right) \delta 7.01-6.94(\mathrm{~m}, 2 \mathrm{H}), 6.86-6.80(\mathrm{~m}, 2 \mathrm{H}), 3.78(\mathrm{~s}, 3 \mathrm{H})$. These spectroscopic data were consistent with those reported in the literature. ${ }^{9}$

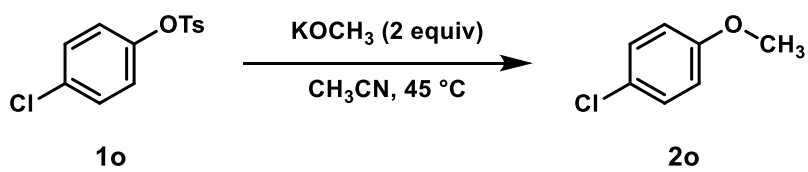

1-Chloro-4-methoxybenzene (2o): The general procedure B was followed. The reaction was performed with 4-chlorophenyl 4-methylbenzenesulfonate 10 (141.3 mg, $0.5 \mathrm{mmol})$, $\mathrm{KOCH}_{3}(70.3 \mathrm{mg}, 1.0 \mathrm{mmol})$ in $2 \mathrm{~mL} \mathrm{CH}{ }_{3} \mathrm{CN}$ for $18.5 \mathrm{~h}$. The desired product $2 \mathrm{o}(53.4 \mathrm{mg}$, $75 \%$ yield) was obtained after purification by silica gel chromatography (2\% EA in PE). ${ }^{1} \mathrm{H}$ NMR $\left(400 \mathrm{MHz}, \mathrm{CDCl}_{3}\right) \delta 7.26-7.21(\mathrm{~m}, 2 \mathrm{H}), 6.86-6.80(\mathrm{~m}, 2 \mathrm{H}), 3.79(\mathrm{~s}, 3 \mathrm{H})$. These spectroscopic data were consistent with those reported in the literature. ${ }^{10}$ 


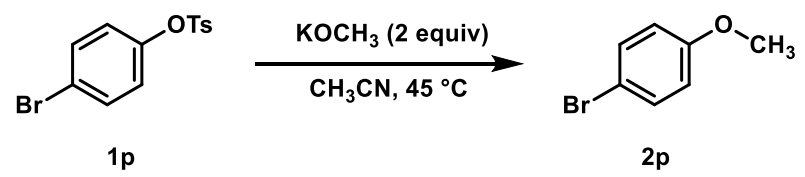

1-Bromo-4-methoxybenzene (2p): The general procedure B was followed. The reaction was performed with 4-bromophenyl 4-methylbenzenesulfonate 1p (64.4 mg, $0.2 \mathrm{mmol})$, $\mathrm{KOCH}_{3}(28.8 \mathrm{mg}, 0.4 \mathrm{mmol})$ in $2 \mathrm{~mL} \mathrm{CH}_{3} \mathrm{CN}$ for $18.5 \mathrm{~h}$. The desired product $\mathbf{2 p}$ (30.2 $\mathrm{mg}$, $82 \%$ yield) was obtained after purification by silica gel chromatography (2\% EA in PE). ${ }^{1} \mathrm{H}$ NMR (400 MHz, $\left.\mathrm{CDCl}_{3}\right) \delta 7.38(\mathrm{~d}, J=9.0 \mathrm{~Hz}, 2 \mathrm{H}), 6.78(\mathrm{~d}, J=9.0 \mathrm{~Hz}, 2 \mathrm{H}), 3.78(\mathrm{~s}$, $3 \mathrm{H})$. These spectroscopic data were consistent with those reported in the literature. ${ }^{10}$

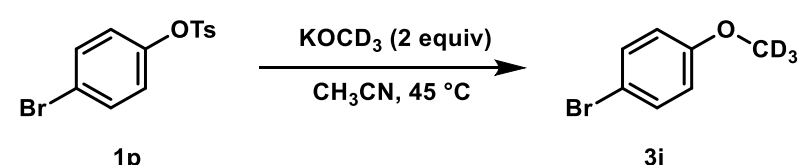

1-Bromo-4-trideuteromethoxybenzene (3j): The general procedure B was followed. The reaction was performed with 4-bromophenyl 4-methylbenzenesulfonate 1p (65.4 mg, 0.2 $\mathrm{mmol}), \mathrm{KOCD}_{3}(29.2 \mathrm{mg}, 0.4 \mathrm{mmol})$ in $2 \mathrm{~mL} \mathrm{CH}_{3} \mathrm{CN}$ for $18.5 \mathrm{~h}$. The desired product $\mathbf{3 j}$ (25.1 mg, 66\% yield) was obtained after purification by silica gel chromatography (2\% EA in PE). ${ }^{1} \mathrm{H}$ NMR (400 MHz, $\left.\mathrm{CDCl}_{3}\right) \delta 7.37(\mathrm{~d}, J=9.1 \mathrm{~Hz}, 2 \mathrm{H}), 6.78(\mathrm{~d}, J=8.4 \mathrm{~Hz}, 2 \mathrm{H})$; ${ }^{13} \mathrm{C} \mathrm{NMR}\left(100 \mathrm{MHz}, \mathrm{CDCl}_{3}\right) \delta 158.8,132.4,115.8,112.9$. These spectroscopic data were consistent with those reported in the literature. ${ }^{11}$

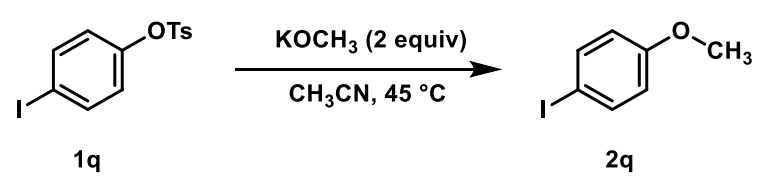

1-Iodo-4-methoxybenzene (2q): The general procedure B was followed. The reaction was performed with 4-iodophenyl 4-methylbenzenesulfonate $\mathbf{1 q}$ (186.2 mg, $0.5 \mathrm{mmol}), \mathrm{KOCH}_{3}$ (69.9 mg, $1.0 \mathrm{mmol}$ ) in $2 \mathrm{~mL} \mathrm{CH} 3 \mathrm{CN}$ for $18.5 \mathrm{~h}$. The desired product $\mathbf{2 q}(85.3 \mathrm{mg}, 73 \%$ yield) was obtained after purification by silica gel chromatography ( $2 \%$ EA in PE). ${ }^{1} \mathrm{H}$ NMR (400 MHz, $\left.\mathrm{CDCl}_{3}\right) \delta 7.56(\mathrm{~d}, J=8.9 \mathrm{~Hz}, 2 \mathrm{H}), 6.68(\mathrm{~d}, J=8.9 \mathrm{~Hz}, 2 \mathrm{H}), 3.78(\mathrm{~s}, 3 \mathrm{H})$. These spectroscopic data were consistent with those reported in the literature. ${ }^{10}$ 
<smiles>Cc1ccc(Br)c(C)c1</smiles>

$1 \mathrm{r}$

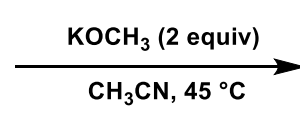

$\mathrm{CH}_{3} \mathrm{CN}, 45^{\circ} \mathrm{C}$<smiles>COc1ccc(C)cc1C</smiles>

$2 \mathbf{r}$

1-Methoxy-2,4-dimethylbenzene (2r): The general procedure B was followed. The reaction was performed with 2,4-dimethylphenyl 4-methylbenzenesulfonate $1 \mathbf{r}(137.9 \mathrm{mg}$, $0.5 \mathrm{mmol}), \mathrm{KOCH}_{3}(70.5 \mathrm{mg}, 1.0 \mathrm{mmol})$ in $2 \mathrm{~mL} \mathrm{CH}_{3} \mathrm{CN}$ for $18.5 \mathrm{~h}$. The desired product 2r (47.6 mg, 70\% yield) was obtained after purification by silica gel chromatography (2\% EA in PE). ${ }^{1} \mathrm{H}$ NMR (400 MHz, $\left.\mathrm{CDCl}_{3}\right) \delta 6.98-6.93(\mathrm{~m}, 2 \mathrm{H}), 6.72(\mathrm{~d}, J=8.8 \mathrm{~Hz}, 1 \mathrm{H})$, $3.80(\mathrm{~s}, 3 \mathrm{H}), 2.26(\mathrm{~s}, 3 \mathrm{H}), 2.19(\mathrm{~s}, 3 \mathrm{H})$. These spectroscopic data were consistent with those reported in the literature. ${ }^{12}$

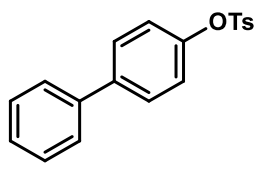

$1 \mathrm{~s}$

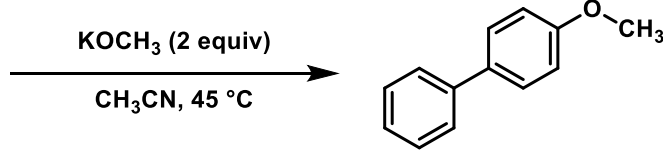

2s

4-Methoxy-1,1'-biphenyl (2s): The general procedure B was followed. The reaction was performed with [1,1'-biphenyl]-4-yl 4-methylbenzenesulfonate $1 \mathrm{~s}(64.8 \mathrm{mg}, 0.2 \mathrm{mmol})$, $\mathrm{KOCH}_{3}(28.0 \mathrm{mg}, 0.4 \mathrm{mmol})$ in $2 \mathrm{~mL} \mathrm{CH}{ }_{3} \mathrm{CN}$ for $18.5 \mathrm{~h}$. The desired product $2 \mathrm{~s}(19.9 \mathrm{mg}$, $54 \%$ yield) was obtained after purification by silica gel chromatography (2\% EA in PE). ${ }^{1} \mathrm{H} \mathrm{NMR}\left(400 \mathrm{MHz}, \mathrm{CDCl}_{3}\right) \delta 7.59-7.50(\mathrm{~m}, 4 \mathrm{H}), 7.42(\mathrm{t}, J=7.6 \mathrm{~Hz}, 2 \mathrm{H}), 7.31(\mathrm{t}, J=7.4$ $\mathrm{Hz}, 1 \mathrm{H}), 6.98(\mathrm{~d}, J=8.9 \mathrm{~Hz}, 2 \mathrm{H}), 3.86(\mathrm{~s}, 3 \mathrm{H})$. These spectroscopic data were consistent with those reported in the literature. ${ }^{13}$

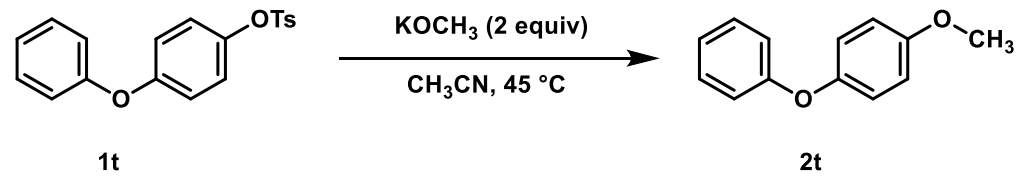

1-Methoxy-4-phenoxybenzene (2t): The general procedure B was followed. The reaction was performed with 4-phenoxyphenyl 4-methylbenzenesulfonate $1 \mathrm{t}$ (170.6 mg, $0.5 \mathrm{mmol})$, $\mathrm{KOCH}_{3}(70.8 \mathrm{mg}, 1.0 \mathrm{mmol})$ in $2 \mathrm{~mL} \mathrm{CH}_{3} \mathrm{CN}$ for $18.5 \mathrm{~h}$. The desired product $\mathbf{2 t}(56.2 \mathrm{mg}$, $56 \%$ yield) was obtained after purification by silica gel chromatography ( $2 \%$ EA in PE). ${ }^{1} \mathrm{H}$ NMR (400 MHz, $\left.\mathrm{CDCl}_{3}\right) \delta 7.35-7.27(\mathrm{~m}, 2 \mathrm{H}), 7.05(\mathrm{td}, J=7.4,1.0 \mathrm{~Hz}, 1 \mathrm{H}), 7.00(\mathrm{~d}$, SI-16 
$J=9.2 \mathrm{~Hz}, 2 \mathrm{H}), 6.96(\mathrm{~d}, J=7.7 \mathrm{~Hz}, 2 \mathrm{H}), 6.89(\mathrm{~d}, J=9.2 \mathrm{~Hz}, 2 \mathrm{H}), 3.81(\mathrm{~s}, 3 \mathrm{H})$. These spectroscopic data were consistent with those reported in the literature. ${ }^{14}$

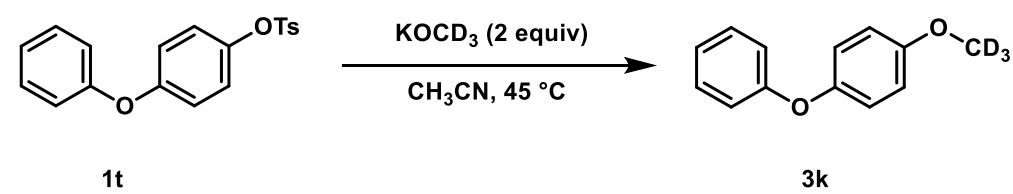

1-Trideuteromethoxy-4-phenoxybenzene (3k): The general procedure B was followed. The reaction was performed with 4-phenoxyphenyl 4-methylbenzenesulfonate 1t (170.8 $\mathrm{mg}, 0.5 \mathrm{mmol}), \mathrm{KOCD}_{3}(73.8 \mathrm{mg}, 1.0 \mathrm{mmol})$ in $2 \mathrm{~mL} \mathrm{CH}_{3} \mathrm{CN}$ for $18.5 \mathrm{~h}$. The desired product 3k (46.9 mg, 46\% yield) was obtained after purification by silica gel chromatography (2\% EA in PE). ${ }^{1} \mathrm{H}$ NMR (400 MHz, $\left.\mathrm{CDCl}_{3}\right) \delta 7.35-7.23(\mathrm{~m}, 2 \mathrm{H}), 7.04$ $(\mathrm{t}, J=7.3 \mathrm{~Hz}, 1 \mathrm{H}), 7.02-6.97(\mathrm{~m}, 2 \mathrm{H}), 6.95(\mathrm{dd}, J=8.8,1.1 \mathrm{~Hz}, 2 \mathrm{H}), 6.89$ (d, $J=9.1 \mathrm{~Hz}$, $2 \mathrm{H}) ;{ }^{13} \mathrm{C} \mathrm{NMR}\left(100 \mathrm{MHz}, \mathrm{CDCl}_{3}\right) \delta 158.7,156.0,150.2,129.7,122.5,121.0,117.7,114.9$; HRMS (APCI+): calc'd for $\mathrm{C}_{13} \mathrm{H}_{10} \mathrm{D}_{3} \mathrm{O}_{2}[\mathrm{M}+\mathrm{H}]^{+}:$204.1098, found: 204.1097

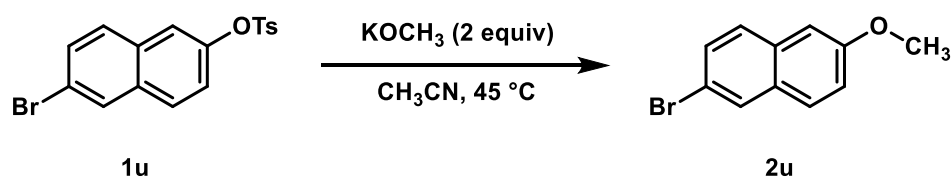

2-Bromo-6-methoxynaphthalene (2u): The general procedure A was followed. The reaction was performed with 6-bromonaphthalen-2-yl 4-methylbenzenesulfonate 1u (30.3 $\mathrm{mg}, 0.08 \mathrm{mmol}), \mathrm{KOCH}_{3}(11.2 \mathrm{mg}, 0.2 \mathrm{mmol})$ in $0.4 \mathrm{~mL} \mathrm{CH}_{3} \mathrm{CN}$. The desired product $2 \mathbf{u}$ (13.2 mg, 70\% yield) was obtained after purification by silica gel chromatography (1\% EA in PE). ${ }^{1} \mathrm{H}$ NMR (600 MHz, $\left.\mathrm{CDCl}_{3}\right) \delta 7.92(\mathrm{~s}, 1 \mathrm{H}), 7.64(\mathrm{~d}, J=8.8 \mathrm{~Hz}, 1 \mathrm{H}), 7.60(\mathrm{~d}, J=$ $8.8 \mathrm{~Hz}, 1 \mathrm{H}), 7.50(\mathrm{~d}, J=8.7 \mathrm{~Hz}, 1 \mathrm{H}), 7.16(\mathrm{~d}, J=8.9 \mathrm{~Hz}, 1 \mathrm{H}), 7.10(\mathrm{~s}, 1 \mathrm{H}), 3.92(\mathrm{~s}, 2 \mathrm{H})$. These spectroscopic data were consistent with those reported in the literature. ${ }^{15}$

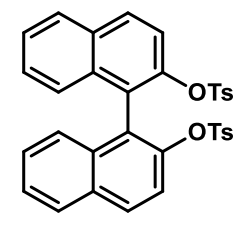

$1 v$

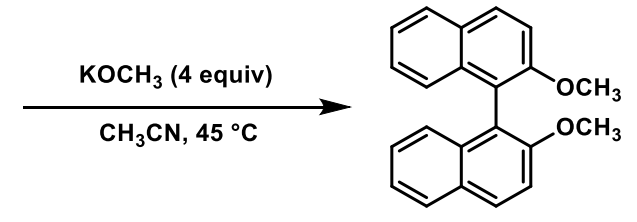

$2 \mathbf{v}$ 
2,2'-Dimethoxy-1,1'-binaphthalene (2v): The general procedure A was followed. The reaction was performed with [1,1'-binaphthalene]-2,2'-diyl bis(4-methylbenzenesulfonate) 1v $(118.9 \mathrm{mg}, 0.2 \mathrm{mmol}), \mathrm{KOCH}_{3}(56.0 \mathrm{mg}, 0.8 \mathrm{mmol})$ in $0.8 \mathrm{~mL} \mathrm{CH}_{3} \mathrm{CN}$. The desired product $2 \mathbf{v}$ (42.0 $\mathrm{mg}, 67 \%$ yield) was obtained after purification by silica gel chromatography (7\% EA in PE). ${ }^{1} \mathrm{H} \mathrm{NMR}\left(400 \mathrm{MHz}, \mathrm{CDCl}_{3}\right) \delta 7.98(\mathrm{~d}, J=9.0 \mathrm{~Hz}, 2 \mathrm{H})$, $7.87(\mathrm{~d}, J=8.1 \mathrm{~Hz}, 2 \mathrm{H}), 7.46(\mathrm{~d}, J=9.0 \mathrm{~Hz}, 2 \mathrm{H}), 7.31(\mathrm{t}, J=7.4 \mathrm{~Hz}, 2 \mathrm{H}), 7.21(\mathrm{t}, J=7.6$ $\mathrm{Hz}, 2 \mathrm{H}), 7.10(\mathrm{~d}, J=8.6 \mathrm{~Hz}, 2 \mathrm{H}), 3.77(\mathrm{~s}, 6 \mathrm{H})$. These spectroscopic data were consistent with those reported in the literature. ${ }^{16}$

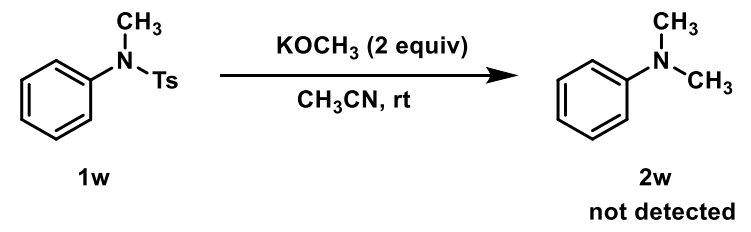

$N, N$-Dimethylaniline (2v): The general procedure A was followed. The reaction was performed with $N$,4-dimethyl- $N$-phenylbenzenesulfonamide $\mathbf{1 v}(131.0 \mathrm{mg}, 0.5 \mathrm{mmol})$, $\mathrm{KOCH}_{3}(77.0 \mathrm{mg}, 1.1 \mathrm{mmol})$ in $2 \mathrm{~mL} \mathrm{CH} 3 \mathrm{CN}$ overnight. The desired product $2 \mathbf{v}$ was not detected.

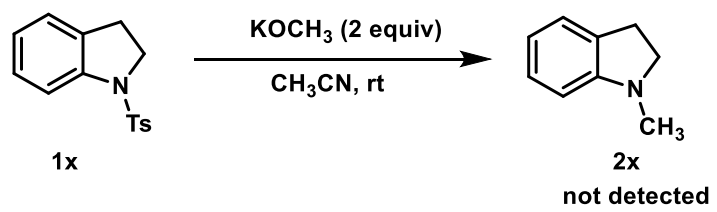

1-Methylindoline (2x): The general procedure A was followed. The reaction was performed with 1-tosylindoline $\mathbf{1 x}(54.7 \mathrm{mg}, 0.2 \mathrm{mmol}), \mathrm{KOCH}_{3}(28.0 \mathrm{mg}, 0.4 \mathrm{mmol})$ in $0.7 \mathrm{~mL} \mathrm{CH}_{3} \mathrm{CN}$ overnight. The desired product $\mathbf{2} \mathbf{x}$ was not detected.

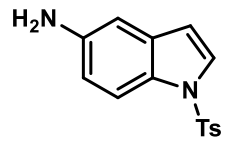

$1 y$

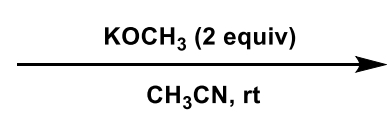

$\mathrm{CH}_{3} \mathrm{CN}, \mathrm{rt}$

1-Methyl-1H-indol-5-amine (2y): The general procedure A was followed. The reaction was performed with 1-tosyl-1H-indol-5-amine $1 \mathbf{y}(57.3 \mathrm{mg}, 0.2 \mathrm{mmol}), \mathrm{KOCH}_{3}(28.0 \mathrm{mg}$, $0.4 \mathrm{mmol})$ in $3.0 \mathrm{~mL} \mathrm{CH} 3 \mathrm{CN}$. The desired product $2 \mathbf{y}(24.4 \mathrm{mg}, 84 \%$ yield) was obtained 
after purification by silica gel chromatography (33\% EA in PE). ${ }^{1} \mathrm{H}$ NMR (600 MHz, $\left.\mathrm{CDCl}_{3}\right) \delta 7.14(\mathrm{~d}, J=8.5 \mathrm{~Hz}, 1 \mathrm{H}), 6.98(\mathrm{~m}, 2 \mathrm{H}), 6.73(\mathrm{~d}, J=8.5 \mathrm{~Hz}, 1 \mathrm{H}), 6.30(\mathrm{~d}, J=3.1$ $\mathrm{Hz}, 1 \mathrm{H}), 3.74(\mathrm{~s}, 3 \mathrm{H})$. These spectroscopic data were consistent with those reported in the literature. ${ }^{17}$

Table S3. Reaction Condition Optimizations for N-Alkylation of Indoles with Alcohols

\begin{tabular}{|c|c|c|c|c|}
\hline entry $^{a}$ & solvent & alcohol (x equiv) & $4 \AA \mathrm{MS}$ & yield \\
\hline 1 & DMF & 2 & w/o & $21 \%^{b}$ \\
\hline 2 & DCE & 2 & w/o & trace \\
\hline 3 & DCM & 2 & w/o & $36 \%^{b}$ \\
\hline 4 & toluene & 2 & w/o & $53 \%^{b}\left(51 \%^{c}\right)$ \\
\hline 5 & $\mathrm{Et}_{2} \mathrm{O}$ & 2 & w/o & $44 \%^{b}$ \\
\hline 6 & DMSO & 2 & w/o & $22 \%^{b}$ \\
\hline 7 & THF & 2 & w/o & $75 \%^{b}\left(60 \%^{c}\right)$ \\
\hline 8 & THF & 2 & $\mathrm{w}$ & $78 \%^{b}\left(67 \%^{c}\right)$ \\
\hline 9 & THF & 1 & $\mathrm{w}$ & $74 \%^{c}$ \\
\hline 10 & THF & 1.1 & $\mathrm{w}$ & $87 \%^{c}$ \\
\hline 11 & THF & 1.2 & $\mathrm{w}$ & $83 \%^{c}$ \\
\hline 12 & THF & 2.5 & $\mathrm{w}$ & $43 \%^{c}$ \\
\hline 13 & THF & 3 & $\mathrm{w}$ & $37 \%{ }^{c}$ \\
\hline
\end{tabular}

${ }^{a}$ Reactions were conducted with $0.2 \mathrm{mmol}$ of $\mathbf{1 d}, 50 \mathrm{mg} 4 \AA \mathrm{MS}$ in $2 \mathrm{~mL}$ of solvent for the suitable time monitored by TLC. ${ }^{b 1} \mathrm{H}$ NMR yield. ${ }^{c}$ Isolated yield. $\mathrm{w}=$ with, $\mathrm{w} / \mathrm{o}=$ without . 
General Procedure for Condition Optimizations (Table S3): To a screw-capped vial equipped with a magnetic stirring bar were added hexanol (x equiv), $\mathrm{KO}^{t} \mathrm{Bu}$ (1.2 equiv), $4 \AA \mathrm{MS}(50 \mathrm{mg})$ and $2 \mathrm{~mL}$ solvent. The mixture was stirred at room temperature for $1 \mathrm{~h}$. Then $N$-tosyl-5-bromoindole $1 d(70.0 \mathrm{mg}, 0.2 \mathrm{mmol})$ was added and stirred at room temperature and the reaction was monitored by TLC until the completion. Then the vial was removed from the glove box. The reaction mixture was filtered through a short pad of Celite, washed with EA (about $4-5 \mathrm{~mL}$ ). The filtrate was concentrated under reduced pressure. Internal standard 1,3,5-trimethoxybenzene (11.2 $\mathrm{mg}, 0.067 \mathrm{mmol})$ was added to the residue and the yield was determined by the crude ${ }^{1} \mathrm{H}$ NMR. Then the desired product was purified by silica gel chromatography (1\% EA in PE).

\section{General Procedure and Spectroscopic Data for N-Alkylation of Indoles} and Heterocyclic Compounds using Alcohols

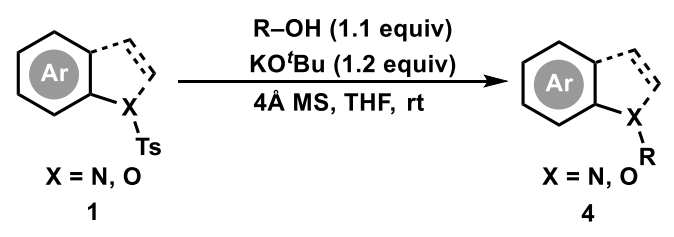

General procedure $C$ : To a dry Schlenk tube or screw-capped vial equipped with a

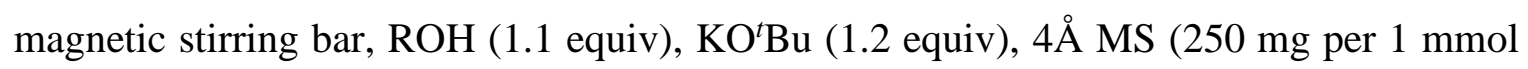
substrate) and solvent $(0.1 \mathrm{mmol} / \mathrm{mL})$ were added under an atmosphere of dry argon. After the mixture was stirred at room temperature for $1 \mathrm{~h}$, substrate 1 ( 1 equiv) were added and stirred at room temperature until the completion of the reaction as monitored by TLC. Then the reaction mixture was filtered through a short pad of Celite, washed with EA ( $5 \mathrm{~mL})$ and concentrated under reduced pressure. The crude mixture was purified by silica gel chromatography to give the desired product.

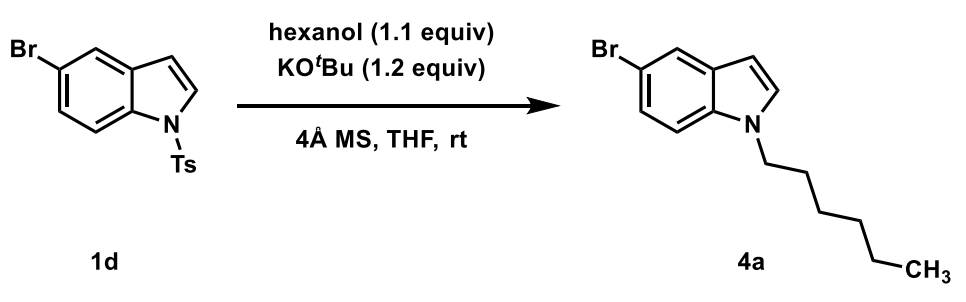


5-Bromo-1-hexyl-1H-indole (4a): The general procedure $\mathrm{C}$ was followed. The reaction was performed with 5-bromo-1-tosyl-1H-indole $1 \mathbf{d}(70.0 \mathrm{mg}, 0.2 \mathrm{mmol})$, hexanol (22.3 mg, $0.22 \mathrm{mmol}), \mathrm{KO}^{t} \mathrm{Bu}(27.2 \mathrm{mg}, 0.24 \mathrm{mmol})$ and $4 \AA \mathrm{MS}(50.0 \mathrm{mg})$ in $2 \mathrm{~mL}$ THF for $1 \mathrm{~h}$. The desired product 4a (48.9 mg, 87\% yield) was obtained after purification by silica gel chromatography (1\% $\mathrm{CH}_{2} \mathrm{Cl}_{2}$ in $\left.\mathrm{PE}\right) .{ }^{1} \mathrm{H} \mathrm{NMR}\left(400 \mathrm{MHz}, \mathrm{CDCl}_{3}\right): \delta 7.75(\mathrm{~s}, 1 \mathrm{H}), 7.31-$ $7.24(\mathrm{~m}, 1 \mathrm{H}), 7.23-7.18(\mathrm{~m}, 1 \mathrm{H}), 7.09$ (d, $J=3.1 \mathrm{~Hz}, 1 \mathrm{H}), 6.42(\mathrm{~d}, J=3.1 \mathrm{~Hz}, 1 \mathrm{H}), 4.08$ $(\mathrm{t}, J=7.1 \mathrm{~Hz}, 2 \mathrm{H}), 1.92-1.75(\mathrm{~m}, 2 \mathrm{H}), 1.35-1.20(\mathrm{~m}, 6 \mathrm{H}), 0.87(\mathrm{t}, J=6.2 \mathrm{~Hz}, 3 \mathrm{H})$. These spectroscopic data were consistent with those reported in the literature. ${ }^{18}$

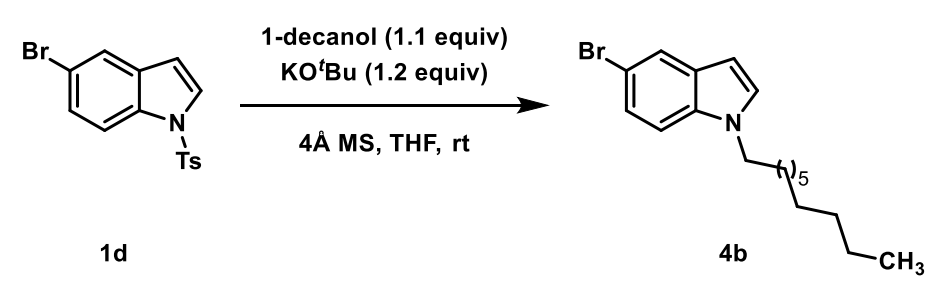

5-Bromo-1-decyl-1H-indole(4b): The general procedure $\mathrm{C}$ was followed. The reaction was performed with 5-bromo-1-tosyl-1H-indole 1 d (176.8 mg, $0.5 \mathrm{mmol})$, 1-decanol (86.9 $\mathrm{mg}, 0.55 \mathrm{mmol}), \mathrm{KO}^{t} \mathrm{Bu}(67.8 \mathrm{mg}, 0.61 \mathrm{mmol})$ and $4 \AA \mathrm{MS}(125.0 \mathrm{mg})$ in $5 \mathrm{~mL}$ THF for 1 h. The desired product $\mathbf{4 b}(153.1 \mathrm{mg}, 90 \%$ yield $)$ was obtained after purification by silica gel chromatography $\left(1 \% \mathrm{CH}_{2} \mathrm{Cl}_{2}\right.$ in PE). ${ }^{1} \mathrm{H} \mathrm{NMR}\left(400 \mathrm{MHz}, \mathrm{CDCl}_{3}\right) \delta 7.74(\mathrm{~d}, J=1.8 \mathrm{~Hz}$, 1H), $\delta 7.30-7.24(\mathrm{~m}, 1 \mathrm{H}), 7.22-7.17(\mathrm{~m}, 1 \mathrm{H}), 7.09(\mathrm{~d}, J=3.1 \mathrm{~Hz}, 1 \mathrm{H}), 6.42(\mathrm{~d}, J=3.1$ $\mathrm{Hz}, 1 \mathrm{H}), 4.08(\mathrm{t}, J=7.1 \mathrm{~Hz}, 2 \mathrm{H}), 1.75-1.86(\mathrm{~m}, 2 \mathrm{H}), 1.26(\mathrm{~m}, 14 \mathrm{H}), 0.88(\mathrm{t}, J=6.8 \mathrm{~Hz}$, $3 \mathrm{H}) ;{ }^{13} \mathrm{C} \mathrm{NMR}\left(100 \mathrm{MHz}, \mathrm{CDCl}_{3}\right) \delta 134.8,130.3,129.1,124.3,123.5,112.6,111.0,100.6$, 46.7, 32.0, 30.3, 29.64, 29.61, 29.40, 29.35, 27.1, 22.8, 14.3; HRMS (APCI+) calc'd for $\mathrm{C}_{18} \mathrm{H}_{27} \mathrm{BrN}[\mathrm{M}+\mathrm{H}]^{+}:$336.1321, found 336.1328.

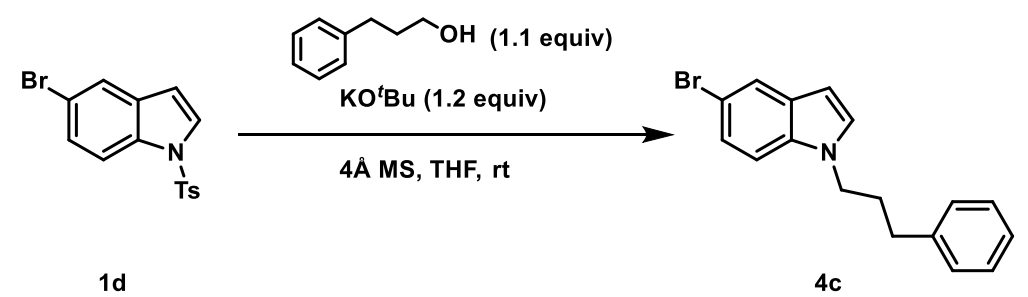

5-Bromo-1-(3-phenylpropyl)-1H-indole (4c): The general procedure C was followed. The reaction was performed with 5-bromo-1-tosyl- $1 H$-indole $1 \mathbf{d}$ (189.7 mg, $0.5 \mathrm{mmol}), 3$ - 
phenylpropan-1-ol (81.1 mg, $0.60 \mathrm{mmol}), \mathrm{KO}^{t} \mathrm{Bu}(74.4 \mathrm{mg}, 0.66 \mathrm{mmol})$ and $4 \AA$ MS (125.0 $\mathrm{mg}$ ) in $5 \mathrm{~mL}$ THF for $1 \mathrm{~h}$. The desired product $4 \mathrm{c}$ (129.7 mg, $76 \%$ yield) was obtained after purification by silica gel chromatography $\left(1 \% \mathrm{CH}_{2} \mathrm{Cl}_{2}\right.$ in $\mathrm{PE}$ to $3 \% \mathrm{CH}_{2} \mathrm{Cl}_{2}$ in $\left.\mathrm{PE}\right) .{ }^{1} \mathrm{H} \mathrm{NMR}$ $\left(400 \mathrm{MHz}, \mathrm{CDCl}_{3}\right) \delta 7.76(\mathrm{~d}, J=1.8 \mathrm{~Hz}, 1 \mathrm{H}), 7.36-7.25(\mathrm{~m}, 3 \mathrm{H}), 7.25-7.18(\mathrm{~m}, 1 \mathrm{H})$, $7.18-7.12(\mathrm{~m}, 3 \mathrm{H}), 7.09(\mathrm{~d}, J=3.1 \mathrm{~Hz}, 1 \mathrm{H}), 6.44(\mathrm{dd}, J=3.1,0.8 \mathrm{~Hz}, 1 \mathrm{H}), 4.10(\mathrm{t}, J=7.1$ $\mathrm{Hz}, 2 \mathrm{H}), 2.62(\mathrm{t}, J=7.6 \mathrm{~Hz}, 2 \mathrm{H}), \delta 2.18$ (quint, $J=7.4 \mathrm{~Hz}, 2 \mathrm{H}) ;{ }^{13} \mathrm{C} \mathrm{NMR}(100 \mathrm{MHz}$, $\left.\mathrm{CDCl}_{3}\right) \delta 140.8,134.8,130.4,129.0,128.7,128.5,126.4,124.4,123.5,112.7,111.0,100.9$, 45.9, 33.0, 31.5; HRMS (APCI+) calc'd for $\mathrm{C}_{17} \mathrm{H}_{17} \mathrm{BrN}[\mathrm{M}+\mathrm{H}]^{+}: 314.0539$, found: 314.0545 .

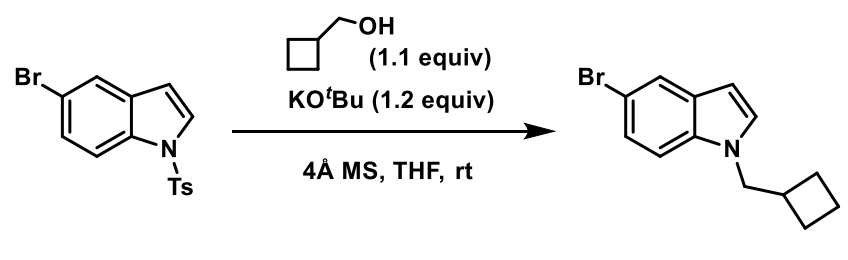

$1 d$

4d

5-Bromo-1-(cyclobutylmethyl)-1H-indole (4d): The general procedure $\mathrm{C}$ was followed. The reaction was performed with 5-bromo-1-tosyl-1H-indole 1 d $(175.3 \mathrm{mg}, 0.5 \mathrm{mmol})$, cyclobutylmethanol $(47.3 \mathrm{mg}, 0.55 \mathrm{mmol}), \mathrm{KO}^{t} \mathrm{Bu}(67.8 \mathrm{mg}, 0.61 \mathrm{mmol})$ and $4 \AA \mathrm{MS}$ (125.0 mg) in $5 \mathrm{~mL}$ THF for $3 \mathrm{~h}$. The desired product $4 \mathbf{d}$ ( $88.3 \mathrm{mg}, 67 \%$ yield) was obtained after purification by silica gel chromatography (100\% PE). ${ }^{1} \mathrm{H}$ NMR $(400 \mathrm{MHz}, \mathrm{CDCl} 3) \delta$ $7.74(\mathrm{~s}, 1 \mathrm{H}), 7.26(\mathrm{~d}, J=8.6 \mathrm{~Hz}, 1 \mathrm{H}), 7.21(\mathrm{~d}, J=8.7 \mathrm{~Hz}, 1 \mathrm{H}), 7.08(\mathrm{~d}, J=3.1 \mathrm{~Hz}, 1 \mathrm{H}), \delta$ $6.41(\mathrm{~d}, J=2.6 \mathrm{~Hz}, 1 \mathrm{H}), 4.08(\mathrm{~d}, J=7.2 \mathrm{~Hz}, 2 \mathrm{H}), 2.81$ (quint, $J=7.6 \mathrm{~Hz}, 1 \mathrm{H}), 2.16-1.97$ $(\mathrm{m}, 2 \mathrm{H}), 1.97-1.68(\mathrm{~m}, 4 \mathrm{H}) ;{ }^{13} \mathrm{C} \mathrm{NMR}\left(100 \mathrm{MHz}, \mathrm{CDCl}_{3}\right) \delta 135.0,130.2,129.1,124.2$, 123.4, 112.6, 111.0, 100.6, 51.8, 36.1, 26.5, 18.3; HRMS (APCI+) calc'd for $\mathrm{C}_{13} \mathrm{H}_{15} \mathrm{BrN}$ $[\mathrm{M}+\mathrm{H}]^{+}:$264.0382, found 264.0378.

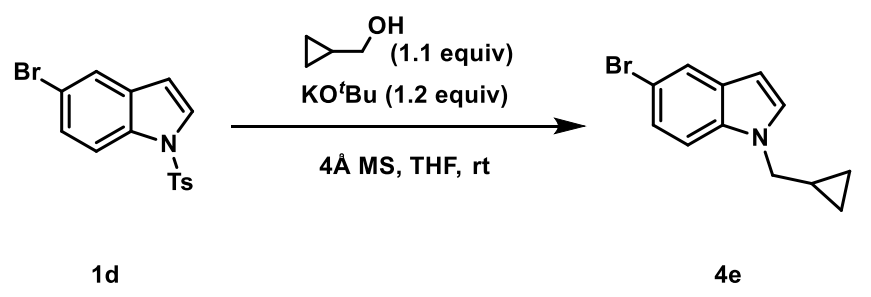


5-Bromo-1-(cyclopropylmethyl)-1H-indole (4e): The general procedure $\mathrm{C}$ was followed. The reaction was performed with 5-bromo-1-tosyl-1H-indole 1 d $(364.0 \mathrm{mg}, 1 \mathrm{mmol})$, cyclopropylmethanol $(82.5 \mathrm{mg}, 1.14 \mathrm{mmol}), \mathrm{KO}^{t} \mathrm{Bu}(141.1 \mathrm{mg}, 1.21 \mathrm{mmol})$ and $4 \AA \mathrm{MS}$ (249.7 $\mathrm{mg}$ ) in $5 \mathrm{~mL}$ THF for $1 \mathrm{~h}$. The desired product $4 \mathrm{e}(191.9 \mathrm{mg}, 74 \%$ yield) was obtained after purification by silica gel chromatography (1\% EA in PE). ${ }^{1} \mathrm{H}$ NMR (400 $\left.\mathrm{MHz}, \mathrm{CDCl}_{3}\right) \delta 7.75(\mathrm{~d}, J=1.8 \mathrm{~Hz}, 1 \mathrm{H}), 7.30-7.26(\mathrm{~m}, 1 \mathrm{H}), 7.19-7.25(\mathrm{~m}, 2 \mathrm{H}), 6.44$ $(\mathrm{dd}, J=3.1,0.6 \mathrm{~Hz}, 1 \mathrm{H}), 3.96(\mathrm{~d}, J=6.8 \mathrm{~Hz}, 2 \mathrm{H}), 1.32-1.17(\mathrm{~m}, 1 \mathrm{H}), 0.70-0.57(\mathrm{~m}$, 2H), $0.30-0.40(\mathrm{~m}, 2 \mathrm{H}) ;{ }^{13} \mathrm{C} \mathrm{NMR}\left(100 \mathrm{MHz}, \mathrm{CDCl}_{3}\right) \delta 134.9,130.3,128.7,124.3,123.5$, 112.7, 111.0, 100.7, 51.0, 11.3, 4.2; HRMS (APCI+) calc'd for $\mathrm{C}_{12} \mathrm{H}_{13} \mathrm{BrN}[\mathrm{M}+\mathrm{H}]^{+}$: 250.0226 , found 250.0230 .

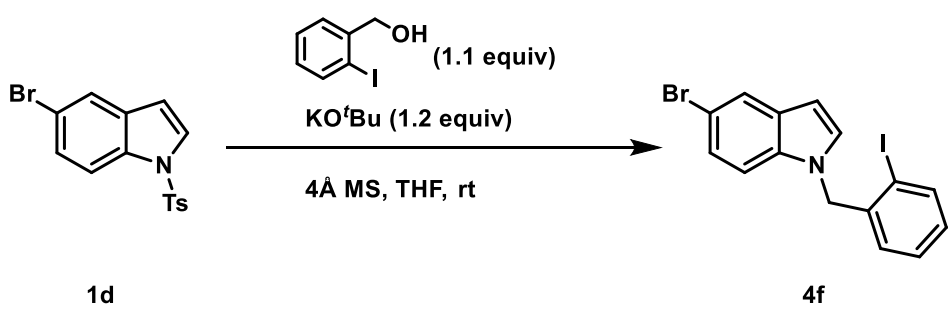

5-Bromo-1-(2-iodobenzyl)-1H-indole (4f): The general procedure $\mathrm{C}$ was followed. The reaction was performed with 5-bromo-1-tosyl-1H-indole 1 d $(175.4 \mathrm{mg}, 0.5 \mathrm{mmol})$, (2iodophenyl)methanol (128.8 mg, $0.55 \mathrm{mmol}), \mathrm{KO}^{t} \mathrm{Bu}(67.8 \mathrm{mg}, 0.61 \mathrm{mmol})$ and $4 \AA \mathrm{AS}$ $(125.0 \mathrm{mg})$ in $5 \mathrm{~mL}$ THF for $3 \mathrm{~h}$. The desired product $\mathbf{4 f}(150.8 \mathrm{mg}, 73 \%$ yield) was obtained after purification by silica gel chromatography (1\% EA in PE). ${ }^{1} \mathrm{H}$ NMR (400 $\left.\mathrm{MHz}, \mathrm{CDCl}_{3}\right) \delta 7.81(\mathrm{~d}, J=7.9 \mathrm{~Hz}, 1 \mathrm{H}), 7.72(\mathrm{~d}, J=1.9 \mathrm{~Hz}, 1 \mathrm{H}), 7.18(\mathrm{dd}, J=8.7,1.8 \mathrm{~Hz}$, 1H), $7.09(\mathrm{td}, J=7.6,1.1 \mathrm{~Hz}, 1 \mathrm{H}), 7.03(\mathrm{~d}, J=3.2 \mathrm{~Hz}, 1 \mathrm{H}), 7.01(\mathrm{~d}, J=8.7 \mathrm{~Hz}, 1 \mathrm{H}), 6.90$ (t, $J=7.3 \mathrm{~Hz}, 1 \mathrm{H}), 6.46(\mathrm{~d}, J=3.1 \mathrm{~Hz}, 1 \mathrm{H}), 6.36(\mathrm{~d}, J=7.7 \mathrm{~Hz}, 1 \mathrm{H}) .5 .19(\mathrm{~s}, 2 \mathrm{H}) ;{ }^{13} \mathrm{C}$ NMR (100 MHz, $\left.\mathrm{CDCl}_{3}\right) \delta 139.6,139.1,135.0,130.4,129.6,128.8,127.6,124.9,123.7$, 113.3, 111.3, 101.8, 97.3, 55.4; HRMS (APCI+) calc'd for $\mathrm{C}_{15} \mathrm{H}_{12} \mathrm{BrIN}[\mathrm{M}+\mathrm{H}]^{+}:$: 411.9192 , found 411.9205 .

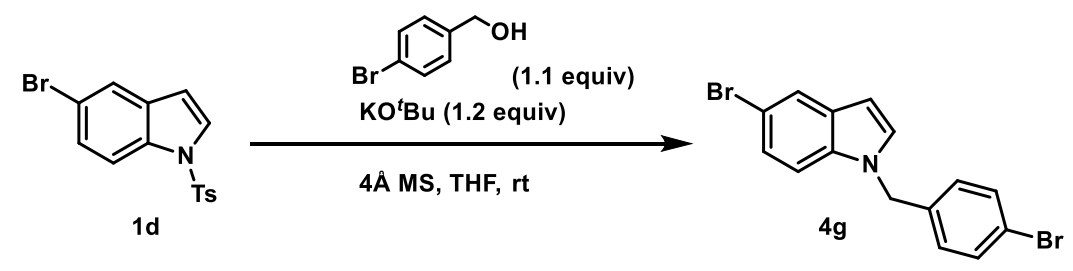


5-Bromo-1-(4-bromobenzyl)-1H-indole (4g): The general procedure $\mathrm{C}$ was followed. The reaction was performed with 5-bromo-1-tosyl- $1 H$-indole $\mathbf{1 d}(175.0 \mathrm{mg}, 0.5 \mathrm{mmol})$, (4-bromophenyl)methanol (104.2 mg, $0.56 \mathrm{mmol}), \mathrm{KO}^{t} \mathrm{Bu}(69.4 \mathrm{mg}, 0.62 \mathrm{mmol})$ and $4 \AA$ MS (129.4 mg) in $5 \mathrm{~mL}$ THF for $3 \mathrm{~h}$. The desired product $\mathbf{4 g}$ (142.5 mg, 78\% yield) was obtained after purification by silica gel chromatography (2\% EA in PE to 5\% EA inPE). ${ }^{1} \mathrm{H}$ NMR (400 MHz, $\left.\mathrm{CDCl}_{3}\right) \delta 7.77(\mathrm{~d}, J=1.8 \mathrm{~Hz}, 1 \mathrm{H}), 7.42(\mathrm{~d}, J=8.5 \mathrm{~Hz}, 2 \mathrm{H}), 7.24(\mathrm{dd}$, $J=8.7,1.8 \mathrm{~Hz}, 1 \mathrm{H}), 7.11(\mathrm{~d}, J=3.2 \mathrm{~Hz}, 1 \mathrm{H}), 7.08(\mathrm{~d}, J=8.7 \mathrm{~Hz}, 1 \mathrm{H}), 6.93(\mathrm{~d}, J=8.6$ $\mathrm{Hz}, 2 \mathrm{H}), 6.50(\mathrm{dd}, J=3.2,0.8 \mathrm{~Hz}, 1 \mathrm{H}), 5.24(\mathrm{~s}, 2 \mathrm{H}) ;{ }^{13} \mathrm{C} \mathrm{NMR}\left(100 \mathrm{MHz}, \mathrm{CDCl}_{3}\right) \delta 136.2$, 134.9, 132.1, 130.6, 129.5, 128.4, 124.8, 123.7, 121.8, 113.2, 111.2, 101.7, 49.8; HRMS (APCI+) calc'd for $\mathrm{C}_{15} \mathrm{H}_{12} \mathrm{Br}_{2} \mathrm{~N}[\mathrm{M}+\mathrm{H}]^{+}:$363.9331, found 363.9338 .

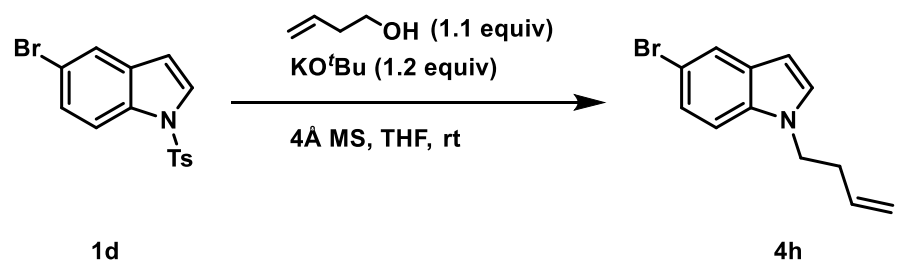

5-Bromo-1-(but-3-en-1-yl)-1H-indole (4h): The general procedure $\mathrm{C}$ was followed. The reaction was performed with 5-bromo-1-tosyl-1H-indole 1 d (175.3 mg, $0.5 \mathrm{mmol})$, but-3en-1-ol (40.2 mg, $0.55 \mathrm{mmol}), \mathrm{KO}^{t} \mathrm{Bu}(67.8 \mathrm{mg}, 0.61 \mathrm{mmol})$ and $4 \AA \mathrm{AS}(125.0 \mathrm{mg})$ in 5 $\mathrm{mL}$ THF for $1 \mathrm{~h}$. The desired product $4 \mathbf{h}(81.3 \mathrm{mg}, 65 \%$ yield $)$ was obtained after purification by silica gel chromatography $\left(1 \% \mathrm{CH}_{2} \mathrm{Cl}_{2}\right.$ in $\mathrm{PE}$ to $2 \% \mathrm{CH}_{2} \mathrm{Cl}_{2}$ in $\left.\mathrm{PE}\right) .{ }^{1} \mathrm{H} \mathrm{NMR}$ $\left(400 \mathrm{MHz}, \mathrm{CDCl}_{3}\right) \delta 7.75(\mathrm{~d}, J=1.3 \mathrm{~Hz}, 1 \mathrm{H}), 7.29(\mathrm{dd}, J=8.7,1.8 \mathrm{~Hz}, 1 \mathrm{H}), 7.22(\mathrm{~d}, J=$ $8.7 \mathrm{~Hz}, 1 \mathrm{H}), 7.10(\mathrm{~d}, J=3.1 \mathrm{~Hz}, 1 \mathrm{H}), 6.42(\mathrm{dd}, J=3.1,0.7 \mathrm{~Hz}, 1 \mathrm{H}), 5.09-5.02(\mathrm{~m}, 2 \mathrm{H})$, $4.16(\mathrm{t}, J=7.1 \mathrm{~Hz}, 2 \mathrm{H}), 2.60-2.52(\mathrm{~m}, 2 \mathrm{H}) ;{ }^{13} \mathrm{C} \mathrm{NMR}\left(100 \mathrm{MHz}, \mathrm{CDCl}_{3}\right) \delta 134.6,134.4$, 130.3, 128.9, 124.3, 123.4, 117.7, 112.6, 110.8, 100.7, 46.2, 34.5; HRMS (APCI+) calc'd for $\mathrm{C}_{12} \mathrm{H}_{13} \mathrm{BrN}[\mathrm{M}+\mathrm{H}]^{+}:$250.0226, found: 250.0229 .

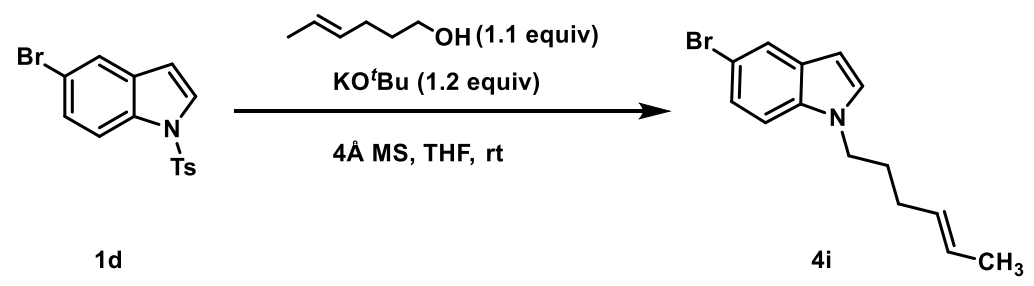


(E)-5-Bromo-1-(hex-4-en-1-yl)-1H-indole (4i): The general procedure C was followed. The reaction was performed with 5-bromo-1-tosyl-1H-indole $1 \mathbf{d}(175.9 \mathrm{mg}, 0.5 \mathrm{mmol})$,

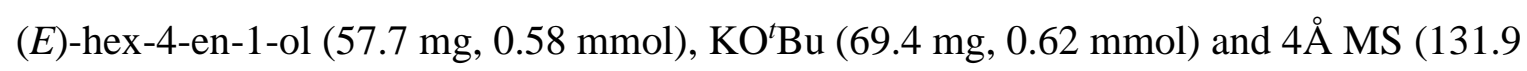
$\mathrm{mg}$ ) in $5 \mathrm{~mL}$ THF for $1 \mathrm{~h}$. The desired product $4 \mathbf{i}$ (102.6 mg, 73\% yield) was obtained after purification by silica gel chromatography (1\% EA in PE). ${ }^{1} \mathrm{H} \mathrm{NMR}\left(400 \mathrm{MHz}, \mathrm{CDCl}_{3}\right) \delta$ $7.75(\mathrm{~d}, J=1.9 \mathrm{~Hz}, 1 \mathrm{H}), 7.28(\mathrm{dd}, J=8.7,1.9 \mathrm{~Hz}, 1 \mathrm{H}), 7.20$ (d, $J=8.7 \mathrm{~Hz}, 1 \mathrm{H}), 7.09$ (d, $J$ $=3.1 \mathrm{~Hz}, 1 \mathrm{H}), 6.43(\mathrm{~d}, J=3.1 \mathrm{~Hz}, 1 \mathrm{H}), 5.68-5.23(\mathrm{~m}, 2 \mathrm{H}), 4.08(\mathrm{t}, J=6.9 \mathrm{~Hz}, 2 \mathrm{H}), 2.03$ $-1.94(\mathrm{~m}, 2 \mathrm{H}), 1.93-1.80(\mathrm{~m}, 2 \mathrm{H}), 1.67(\mathrm{~d}, J=5.2 \mathrm{~Hz}, 3 \mathrm{H}) ;{ }^{13} \mathrm{C} \mathrm{NMR}\left(100 \mathrm{MHz}, \mathrm{CDCl}_{3}\right)$ $\delta 134.8,130.4,129.7,129.1,126.5,124.2,123.5,112.6,111.0,101.0,45.9,29.9,29.8,18.1$; HRMS (APCI+) calc'd for $\mathrm{C}_{14} \mathrm{H}_{17} \mathrm{BrN}[\mathrm{M}+\mathrm{H}]^{+}:$278.0539, found: 278.0545.

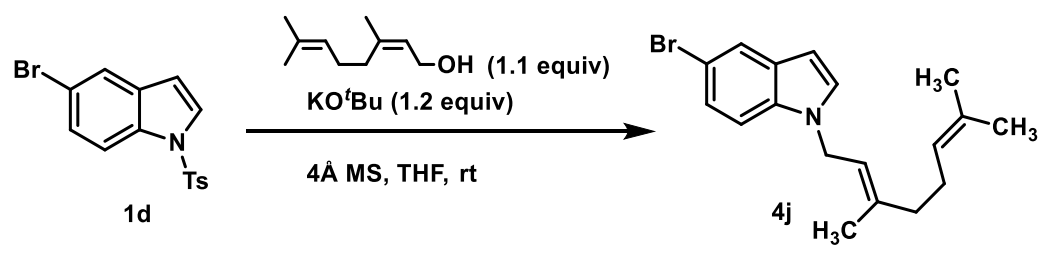

(E)-5-Bromo-1-(3,7-dimethylocta-2,6-dien-1-yl)-1H-indole (4j): The general procedure $\mathrm{C}$ was followed. The reaction was performed with 5-bromo-1-tosyl-1H-indole 1d (175.1 mg, 0.5 mmol), (Z)-3,7-dimethylocta-2,6-dien-1-ol (89.0 mg, 0.58 mmol), KO ${ }^{t} \mathrm{Bu}(70.3 \mathrm{mg}$, $0.62 \mathrm{mmol})$ and $4 \AA \mathrm{MS}(131.8 \mathrm{mg})$ in $5 \mathrm{~mL}$ THF for $5 \mathrm{~h}$. The desired product $\mathbf{4 j}$ ( $78.0 \mathrm{mg}$, $47 \%$ yield) was obtained after purification by silica gel chromatography (1\% EA in PE). ${ }^{1} \mathrm{H}$ NMR $\left(400 \mathrm{MHz}, \mathrm{CDCl}_{3}\right) \delta 7.74(\mathrm{~d}, J=1.8 \mathrm{~Hz}, 1 \mathrm{H}), 7.26(\mathrm{dd}, J=8.7,1.8 \mathrm{~Hz}, 1 \mathrm{H}), 7.19$ $(\mathrm{d}, J=8.7 \mathrm{~Hz}, 1 \mathrm{H}), 7.10(\mathrm{~d}, J=3.1 \mathrm{~Hz}, 1 \mathrm{H}), 6.41(\mathrm{~d}, J=3.1 \mathrm{~Hz}, 1 \mathrm{H}), 5.35(\mathrm{t}, J=6.8 \mathrm{~Hz}$, $1 \mathrm{H}), 5.14(\mathrm{t}, J=7.1 \mathrm{~Hz}, 1 \mathrm{H}), 4.66(\mathrm{dd}, J=6.8,1.3 \mathrm{~Hz}, 2 \mathrm{H}), 2.28-2.20(\mathrm{~m}, 2 \mathrm{H}), 2.20-$ $2.12(\mathrm{~m}, 2 \mathrm{H}), 1.77(\mathrm{~d}, J=1.4 \mathrm{~Hz}, 3 \mathrm{H}), 1.72(\mathrm{~s}, 3 \mathrm{H}), 1.63(\mathrm{~s}, 3 \mathrm{H}) ;{ }^{13} \mathrm{C}$ NMR $(100 \mathrm{MHz}$, $\left.\mathrm{CDCl}_{3}\right) \delta 140.4,134.7,132.6,130.5,128.7,124.2,123.6,123.4,120.3,112.7,111.1,100.7$, 44.2, 32.3, 26.5, 25.9, 23.4, 17.9; HRMS (APCI+) calc'd for $\mathrm{C}_{18} \mathrm{H}_{23} \mathrm{BrN}[\mathrm{M}+\mathrm{H}]^{+}: 332.1008$, found: 332.1010 .
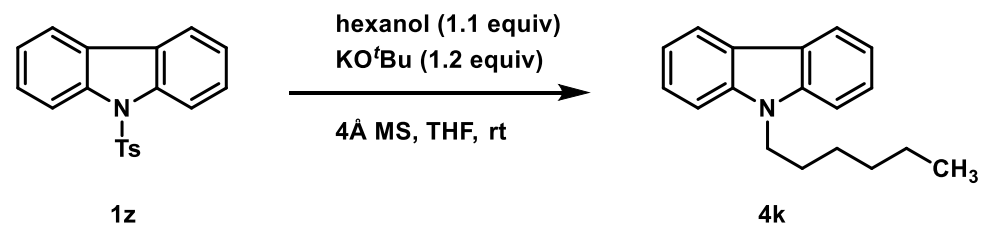
9-Hexyl-9H-carbazole (4k): The general procedure $\mathrm{C}$ was followed. The reaction was performed with 9-tosyl-9H-carbazole $1 \mathrm{z}(161.0 \mathrm{mg}, 0.5 \mathrm{mmol})$, hexanol (55.3 mg, 0.55 $\mathrm{mmol}), \mathrm{KO}^{t} \mathrm{Bu}(68.2 \mathrm{mg}, 0.61 \mathrm{mmol})$ and $4 \AA \mathrm{MS}(128.3 \mathrm{mg})$ in $5 \mathrm{~mL}$ THF for $5 \mathrm{~h}$. The desired product $4 \mathbf{k}(80.1 \mathrm{mg}$, 64\% yield) was obtained after purification by silica gel chromatography (1\% EA in PE). ${ }^{1} \mathrm{H} \mathrm{NMR}\left(400 \mathrm{MHz}, \mathrm{CDCl}_{3}\right) \delta 8.10(\mathrm{~d}, J=7.7 \mathrm{~Hz}, 2 \mathrm{H})$, $7.53-7.34(\mathrm{~m}, 4 \mathrm{H}), 7.25-7.20(\mathrm{~m}, 2 \mathrm{H}), 4.30(\mathrm{t}, J=7.3 \mathrm{~Hz}, 2 \mathrm{H}), 1.87$ (quint, $J=7.4 \mathrm{~Hz}$, $2 \mathrm{H}), 1.49-1.17(\mathrm{~m}, 6 \mathrm{H}), 0.86(\mathrm{t}, J=7.1 \mathrm{~Hz}, 3 \mathrm{H})$. These spectroscopic data were consistent with those reported in the literature. ${ }^{19}$

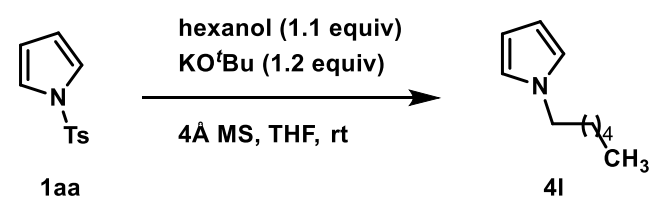

1-Hexyl-1H-pyrrole (4l): The general procedure $\mathrm{C}$ was followed. The reaction was performed with 1-tosyl-1H-pyrrole 1aa ( $88.3 \mathrm{mg}, 0.4 \mathrm{mmol})$, hexanol (44.8 mg, 0.44 $\mathrm{mmol}), \mathrm{KO}^{t} \mathrm{Bu}(54.4 \mathrm{mg}, 0.48 \mathrm{mmol})$ and $4 \AA \mathrm{MS}(100.6 \mathrm{mg})$ in $4 \mathrm{~mL}$ THF for $2.5 \mathrm{~h}$. The desired product $4 \mathrm{l}$ (36.2 $\mathrm{mg}$, 60\% yield) was obtained after purification by silica gel chromatography (1\% EA in PE, the product is volatile and dried under vacuum at $\left.20{ }^{\circ} \mathrm{C}\right) .{ }^{1} \mathrm{H} \mathrm{NMR}\left(400 \mathrm{MHz}, \mathrm{CDCl}_{3}\right) \delta 6.65(\mathrm{t}, J=2.1 \mathrm{~Hz}, 2 \mathrm{H}), 6.14(\mathrm{t}, J=2.1 \mathrm{~Hz}, 2 \mathrm{H})$, $3.87(\mathrm{t}, J=7.2 \mathrm{~Hz}, 2 \mathrm{H}), 1.83-1.71(\mathrm{~m}, 2 \mathrm{H}), 1.36-1.24(\mathrm{~m}, 6 \mathrm{H}), 0.88(\mathrm{t}, J=6.7 \mathrm{~Hz}$, $3 \mathrm{H}) ;{ }^{13} \mathrm{C} \mathrm{NMR}\left(100 \mathrm{MHz}, \mathrm{CDCl}_{3}\right) \delta 120.6,107.9,49.8,31.7,31.5,26.6,22.7,14.2$; HRMS (APCI+) calc'd for $\mathrm{C}_{10} \mathrm{H}_{18} \mathrm{~N}[\mathrm{M}+\mathrm{H}]^{+}:$152.1434, found: 152.1435 .

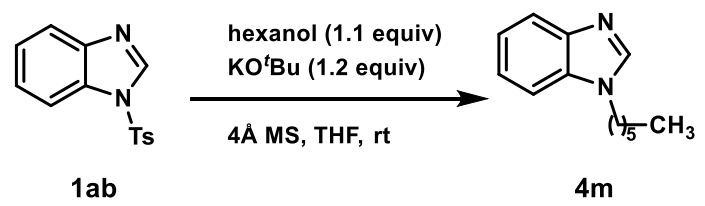

1-Hexyl-1H-benzo $[\boldsymbol{d}]$ imidazole $(\mathbf{4 m})$ : The general procedure $\mathrm{C}$ was followed. The reaction was performed with 1-tosyl-1H-benzo[ $d]$ imidazole 1ab $(109.0 \mathrm{mg}, 0.4$ $\mathrm{mmol})$, hexanol (45.9 mg, $0.44 \mathrm{mmol}), \mathrm{KO}^{t} \mathrm{Bu}(55.4 \mathrm{mg}, 0.48 \mathrm{mmol})$ and $4 \AA \mathrm{MS}$ $(100.1 \mathrm{mg})$ in $4 \mathrm{~mL}$ THF for $2 \mathrm{~h}$. The desired product $\mathbf{4 m}(40.1 \mathrm{mg}, 50 \%$ yield) was obtained after purification by silica gel chromatography (40\% EA in PE to 50\% EA 
in PE). ${ }^{1} \mathrm{H}$ NMR (400 MHz, $\left.\mathrm{CDCl}_{3}\right) \delta 7.90(\mathrm{~s}, 1 \mathrm{H}), 7.81(\mathrm{dd}, J=6.4,2.3 \mathrm{~Hz}, 1 \mathrm{H}), 7.40$ (dd, $J=6.5,2.3 \mathrm{~Hz}, 1 \mathrm{H}$ ), $7.35-7.23(\mathrm{~m}, 2 \mathrm{H}), 4.16(\mathrm{t}, J=7.2 \mathrm{~Hz}, 2 \mathrm{H}$ ), 1.87 (quint, $J$ $=7.2 \mathrm{~Hz}, 2 \mathrm{H}), 1.41-1.14(\mathrm{~m}, 6 \mathrm{H}), 0.86(\mathrm{t}, J=7.0 \mathrm{~Hz}, 3 \mathrm{H}) ;{ }^{13} \mathrm{C} \mathrm{NMR}(100 \mathrm{MHz}$, $\left.\mathrm{CDCl}_{3}\right) \delta 143.8,143.0,133.9,122.9,122.2,120.4,109.8,45.3,31.4,29.9,26.6,22.6$, 14.1; HRMS (ESI+) calc'd for $\mathrm{C}_{13} \mathrm{H}_{19} \mathrm{~N}_{2}[\mathrm{M}+\mathrm{H}]^{+}:$203.1543, found: 203.1548 .

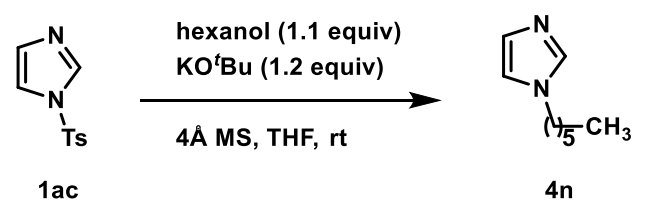

1-Hexyl-1H-imidazole (4n): The general procedure $\mathrm{C}$ was followed. The reaction was performed with 1-tosyl-1H-imidazole 1ac $(89.0 \mathrm{mg}, 0.4 \mathrm{mmol})$, hexanol $(44.7 \mathrm{mg}, 0.44$ $\mathrm{mmol}), \mathrm{KO}{ }^{t} \mathrm{Bu}(54.3 \mathrm{mg}, 0.48 \mathrm{mmol})$ and $4 \AA \mathrm{MS}(100.5 \mathrm{mg})$ in $4 \mathrm{~mL}$ THF for $2 \mathrm{~h}$. The desired product $4 \mathbf{n}$ ( $25.2 \mathrm{mg}$, 41\% yield) was obtained after purification by silica gel chromatography (100\% DCM to 50\% EA in DCM, the product is volatile and dried under vacuum at $-20{ }^{\circ} \mathrm{C}$ ). ${ }^{1} \mathrm{H} \mathrm{NMR}\left(400 \mathrm{MHz}, \mathrm{CDCl}_{3}\right) \delta 7.47$ (s, $\left.1 \mathrm{H}\right), 7.04(\mathrm{~s}, 1 \mathrm{H}), 6.90(\mathrm{~s}, 1 \mathrm{H})$, $3.91(\mathrm{t}, J=7.2 \mathrm{~Hz}, 2 \mathrm{H}), 1.89-1.68(\mathrm{~m}, 2 \mathrm{H}), 1.41-1.11(\mathrm{~m}, 6 \mathrm{H}), 0.87(\mathrm{t}, J=6.7 \mathrm{~Hz}, 3 \mathrm{H})$; ${ }^{13} \mathrm{C}$ NMR $\left(100 \mathrm{MHz}, \mathrm{CDCl}_{3}\right) \delta 137.1,129.3,118.9,47.2,31.4,31.2,26.3,22.6,14.1$; HRMS (ESI+) calc'd for $\mathrm{C}_{9} \mathrm{H}_{17} \mathrm{~N}_{2}[\mathrm{M}+\mathrm{H}]^{+}:$153.1386, found: 153.1382 .

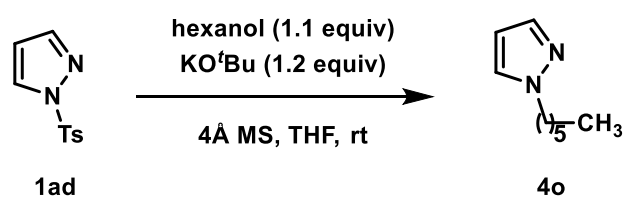

1-Hexyl-1H-pyrazole (4o): The general procedure $\mathrm{C}$ was followed. The reaction was performed with 1-tosyl-1H-pyrazole 1ad $(88.8 \mathrm{mg}, 0.4 \mathrm{mmol})$, hexanol $(45.4 \mathrm{mg}, 0.44$ $\mathrm{mmol}), \mathrm{KO} \mathrm{Bu}^{\prime}(54.6 \mathrm{mg}, 0.48 \mathrm{mmol})$ and $4 \AA \mathrm{MS}(100.1 \mathrm{mg})$ in $4 \mathrm{~mL}$ THF for $2.5 \mathrm{~h}$. The desired product 40 (51.0 $\mathrm{mg}, 84 \%$ yield) was obtained after purification by silica gel chromatography $\left(1 \% \mathrm{EA}\right.$ in $\mathrm{PE}$, the product is volatile and dried under vacuum at $\left.-20^{\circ} \mathrm{C}\right)$. ${ }^{1} \mathrm{H} \mathrm{NMR}\left(400 \mathrm{MHz}, \mathrm{CDCl}_{3}\right) \delta 7.50(\mathrm{~d}, J=1.4 \mathrm{~Hz}, 1 \mathrm{H}), 7.37(\mathrm{~d}, J=2.1 \mathrm{~Hz}, 1 \mathrm{H}), 6.23(\mathrm{t}, J$ $=2.0 \mathrm{~Hz}, 1 \mathrm{H}), 4.12(\mathrm{t}, J=7.2 \mathrm{~Hz}, 2 \mathrm{H}), 1.90-1.79(\mathrm{~m}, 2 \mathrm{H}), 1.36-1.15(\mathrm{~m}, 6 \mathrm{H}), 0.87(\mathrm{t}, J$ 
$=6.8 \mathrm{~Hz}, 3 \mathrm{H}) ;{ }^{13} \mathrm{C} \mathrm{NMR}\left(100 \mathrm{MHz}, \mathrm{CDCl}_{3}\right) \delta 139.1,129.0,105.3,52.3,31.4,30.5,26.4$, 22.6, 14.1; HRMS (ESI+) calc'd for $\mathrm{C}_{9} \mathrm{H}_{17} \mathrm{~N}_{2}[\mathrm{M}+\mathrm{H}]^{+}$: 153.1386, found: 153.1394 .

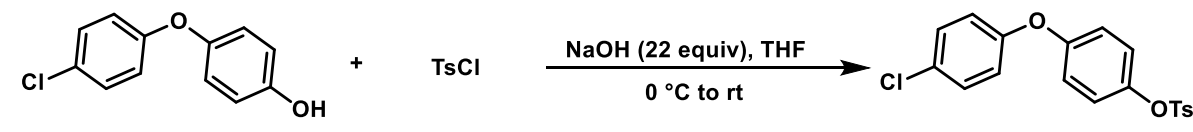

4-(4-Chlorophenoxy)phenyl 4-methylbenzenesulfonate (1ae) ${ }^{20}$ : To a dry Schlenk flask equipped with a magnetic stirring bar, 4-(4-chlorophenoxy)phenol (1.10 g, $5 \mathrm{mmol}), \mathrm{NaOH}$ (4.4 g, $110 \mathrm{mmol})$ and $10 \mathrm{ml}$ of THF were added under an atmosphere of dry argon and stirred for 15 minutes at room temperature. Then $\mathrm{TsCl}(1.05 \mathrm{~g}, 5.5 \mathrm{mmol}, 1.1$ equiv, in 10 $\mathrm{mL}$ THF) was slowly added within $15 \mathrm{~min}$ at $0{ }^{\circ} \mathrm{C}$. The reaction mixture was stirred at room temperature for $12 \mathrm{~h}$, and then quenched with $\mathrm{H}_{2} \mathrm{O}(20 \mathrm{~mL})$ and extracted with EA $(20 \mathrm{~mL} \times 3)$. The combined organic layers were washed with brine and dried over anhydrous $\mathrm{Na}_{2} \mathrm{SO}_{4}$. The solvents were removed under reduced pressure. Then the desired product 1ae (1.29 g, 69\% yield) was obtained after purification by silica gel chromatography (10\% EA in PE). ${ }^{1} \mathrm{H} \mathrm{NMR}\left(400 \mathrm{MHz}, \mathrm{CDCl}_{3}\right) \delta 7.72(\mathrm{~d}, J=8.3 \mathrm{~Hz}, 2 \mathrm{H})$, $7.43-7.11(\mathrm{~m}, 4 \mathrm{H}), 7.11-6.53(\mathrm{~m}, 6 \mathrm{H}), 2.45(\mathrm{~s}, 3 \mathrm{H}) .{ }^{13} \mathrm{C} \mathrm{NMR}\left(100 \mathrm{MHz}, \mathrm{CDCl}_{3}\right) \delta 155.8$, 155.3, 145.6, 145.1, 132.3, 130.0, 129.9, 129.0, 128.67, 124.0, 120.5, 119.4, 21.9. HRMS (ESI+) calc'd for $\mathrm{C}_{19} \mathrm{H}_{15} \mathrm{ClSO}_{4} \mathrm{Na}[\mathrm{M}+\mathrm{Na}]^{+}:$397.0272, found: 397.0284.
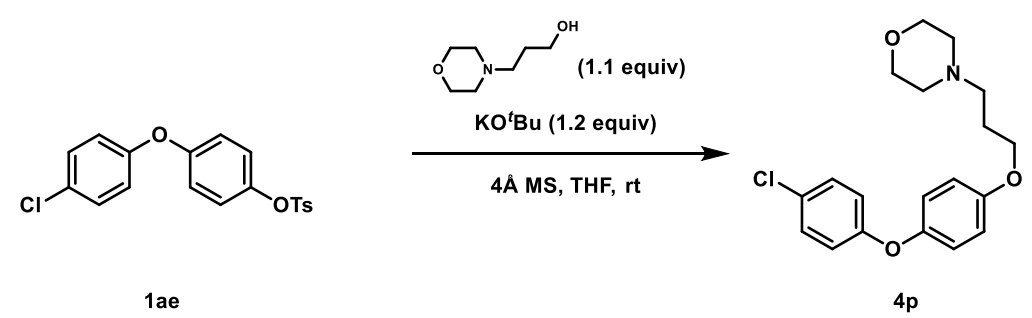

4-(3-(4-(4-Chlorophenoxy)phenoxy)propyl)morpholine (4p): The general procedure C was followed. The reaction was performed with 4-(4-chlorophenoxy)phenyl 4methylbenzenesulfonate 1ae (74.8 mg, $0.2 \mathrm{mmol})$, 3-morpholinopropan-1-ol (86.9 mg, $0.22 \mathrm{mmol}), \mathrm{KO}^{t} \mathrm{Bu}(26.9 \mathrm{mg}, 0.24 \mathrm{mmol})$ and $4 \AA \mathrm{MS}(200.0 \mathrm{mg})$ in $2 \mathrm{~mL}$ THF for $12 \mathrm{~h}$. The desired product $4 \mathbf{p}$ (38.4 mg, 55\% yield) was obtained after purification by silica gel chromatography (rinsed the silica gel with $0.2 \% \mathrm{Et}_{3} \mathrm{~N}$ in $\mathrm{PE}$, then with $25 \% \mathrm{EA}$ in PE as 
the eluent). ${ }^{1} \mathrm{H}$ NMR (400 MHz, $\left.\mathrm{CDCl}_{3}\right) \delta 7.24(\mathrm{~d}, J=9.0 \mathrm{~Hz}, 2 \mathrm{H}), 6.95(\mathrm{~d}, J=9.1 \mathrm{~Hz}$, 2H), $6.91-6.82(\mathrm{~m}, 4 \mathrm{H}), 4.00(\mathrm{t}, J=6.3 \mathrm{~Hz}, 2 \mathrm{H}), 3.73(\mathrm{t}, J=4.7 \mathrm{~Hz}, 4 \mathrm{H}), 2.61-2.36(\mathrm{~m}$, 6H), $2.03-1.93(\mathrm{~m}, 2 \mathrm{H}) ;{ }^{13} \mathrm{C} \mathrm{NMR}\left(100 \mathrm{MHz}, \mathrm{CDCl}_{3}\right) \delta 157.3,155.6,149.8,129.7,127.4$, 120.9, 118.8, 115.7, 67.1, 66.7, 55.7, 53.9, 26.6; HRMS (ESI+) calc'd for $\mathrm{C}_{19} \mathrm{H}_{23} \mathrm{ClNO}_{3}$ $[\mathrm{M}+\mathrm{H}]^{+}:$348.1361, found: 348.1366.

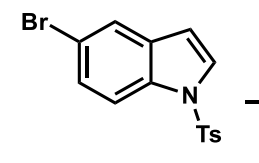

$1 d$

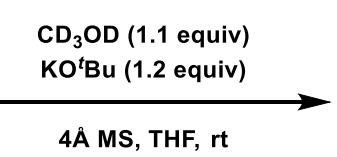

4Å MS, THF, rt

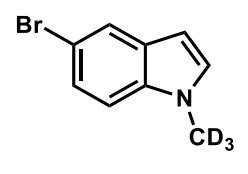

$3 d$

5-Bromo-1-trideuteromethyl-1H-indole (3d): The general procedure $\mathrm{C}$ was followed. The reaction was performed with 5-bromo-1-tosyl-1H-indole $1 \mathbf{d}$ (175.1 mg, $0.5 \mathrm{mmol})$, $\mathrm{CD}_{3} \mathrm{OD}(19.9 \mathrm{mg}, 0.55 \mathrm{mmol}), \mathrm{KO}^{t} \mathrm{Bu}(67.2 \mathrm{mg}, 0.60 \mathrm{mmol})$ and $4 \AA \mathrm{MS}(200.0 \mathrm{mg})$ in 5 $\mathrm{mL}$ THF for $3 \mathrm{~h}$. The desired product 3d (103.9 mg, 98\% yield) was obtained after purification by silica gel chromatography (5\% EA in PE).

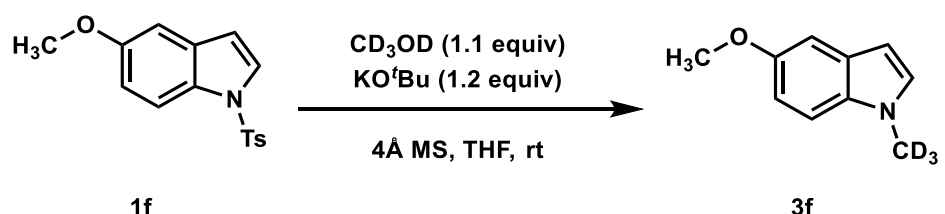

5-Methoxy-1-trideuteromethyl-1H-indole (3f): The general procedure C was followed. The reaction was performed with 5-methoxy-1-tosyl-1H-indole $\mathbf{1 f}(60.3 \mathrm{mg}, 0.2 \mathrm{mmol})$, $\mathrm{CD}_{3} \mathrm{OD}(8.0 \mathrm{mg}, 0.22 \mathrm{mmol}), \mathrm{KO}^{t} \mathrm{Bu}(26.9 \mathrm{mg}, 0.24 \mathrm{mmol})$ and $4 \AA \mathrm{MS}(100.0 \mathrm{mg})$ in 3 $\mathrm{mL}$ THF overnight. The desired product 3 f (28.3 $\mathrm{mg}, 86 \%$ yield) was obtained after purification by silica gel chromatography (5\% EA in PE).

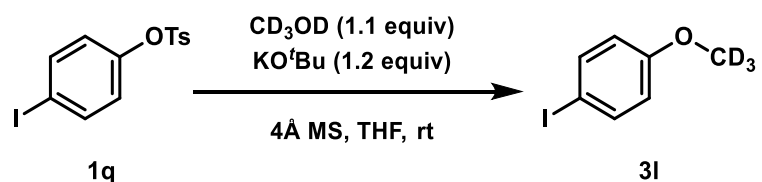

1-Iodo-4-trideuteromethoxybenzene (31): The general procedure $\mathrm{C}$ was followed. The reaction was performed with, 4-iodophenyl 4-methylbenzenesulfonate 1q (74.6 mg, 0.2 $\mathrm{mmol}), \mathrm{CD}_{3} \mathrm{OD}(8.0 \mathrm{mg}, 0.11 \mathrm{mmol}), \mathrm{KO}^{t} \mathrm{Bu}(26.9 \mathrm{mg}, 0.24 \mathrm{mmol})$ and $4 \AA \mathrm{AS}(100 \mathrm{mg})$ 
in $3 \mathrm{~mL}$ THF overnight. The desired product 31 ( $28.3 \mathrm{mg}$, $60 \%$ yield) was obtained after purification by silica gel chromatography (5\% EA in PE). ${ }^{1} \mathrm{H}$ NMR $\left(400 \mathrm{MHz}, \mathrm{CDCl}_{3}\right) \delta$ $7.58-7.53(\mathrm{~m}, 2 \mathrm{H}), 6.71-6.65(\mathrm{~m}, 2 \mathrm{H}) ;{ }^{13} \mathrm{C} \mathrm{NMR}\left(100 \mathrm{MHz}, \mathrm{CDCl}_{3}\right) \delta 159.6,138.3$, 116.5, 82.8; HRMS (APCI+): calc'd for $\mathrm{C}_{7} \mathrm{H}_{4} \mathrm{D}_{3} \mathrm{IO}[\mathrm{M}+\mathrm{H}]^{+}:$237.9803, found: 237.9802 .

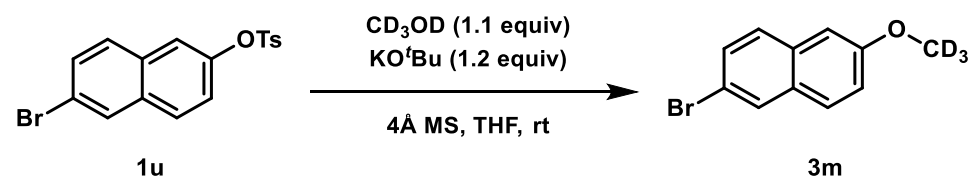

2-bromo-6-trideuteromethoxynaphthalene (3m): The general procedure $\mathrm{C}$ was followed. The reaction was performed with 6-bromonaphthalen-2-yl 4-methylbenzenesulfonate 1u (75.5 mg, $0.2 \mathrm{mmol}), \mathrm{CD}_{3} \mathrm{OD}(8.0 \mathrm{mg}, 0.11 \mathrm{mmol}), \mathrm{KO}^{t} \mathrm{Bu}(26.9 \mathrm{mg}, 0.24 \mathrm{mmol})$ and $4 \AA$ MS (100 mg) in $3 \mathrm{~mL}$ THF overnight. The desired product 3m (27.4 mg, 57\% yield) was obtained after purification by silica gel chromatography (5\% EA in PE). ${ }^{1} \mathrm{H}$ NMR (400 $\left.\mathrm{MHz}, \mathrm{CDCl}_{3}\right) \delta 7.92(\mathrm{~d}, J=2.0 \mathrm{~Hz}, 1 \mathrm{H}), 7.65(\mathrm{~d}, J=9.0 \mathrm{~Hz}, 1 \mathrm{H}), 7.61(\mathrm{~d}, J=8.8 \mathrm{~Hz}, 1 \mathrm{H})$, $7.50(\mathrm{dd}, J=8.7,2.0 \mathrm{~Hz}, 1 \mathrm{H}), 7.16(\mathrm{dd}, J=9.0,2.5 \mathrm{~Hz}, 1 \mathrm{H}), 7.09(\mathrm{~d}, J=2.5 \mathrm{~Hz}, 1 \mathrm{H}) ;{ }^{13} \mathrm{C}$ NMR (100 MHz, $\left.\mathrm{CDCl}_{3}\right) \delta 158.0,133.2,130.1,129.8,129.7,128.6,128.5,119.9,117.1$, 105.8; HRMS (APCI+): calc'd for $\mathrm{C}_{11} \mathrm{H}_{6} \mathrm{D}_{3} \mathrm{BrO}[\mathrm{M}+\mathrm{H}]^{+}:$240.0097, found: 240.0103.

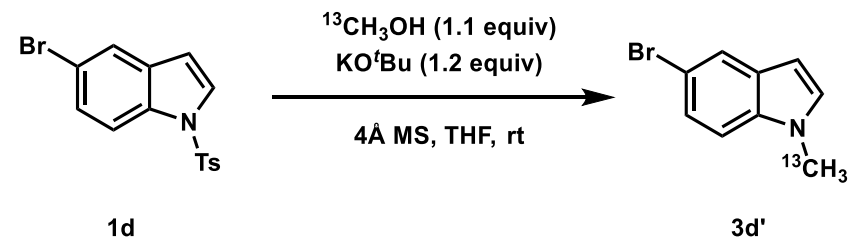

5-Bromo-1-methyl-1H-indole (3d'): The general procedure $\mathrm{C}$ was followed. The reaction was performed with 5-bromo-1-tosyl- $1 H$-indole $1 d(71.1 \mathrm{mg}, 0.2 \mathrm{mmol}),{ }^{13} \mathrm{CH}_{3} \mathrm{OH}(7.8$ $\mathrm{mg}, 0.11 \mathrm{mmol}), \mathrm{KO}^{t} \mathrm{Bu}(26.9 \mathrm{mg}, 0.24 \mathrm{mmol})$ and $4 \AA \mathrm{AS}(80.0 \mathrm{mg})$ in $3 \mathrm{~mL}$ THF for 3 h. The desired product 3d' (42.4 mg, 99\% yield) was obtained after purification by silica gel chromatography (5\% EA in PE). ${ }^{1} \mathrm{H}$ NMR (400 MHz, $\left.\mathrm{CDCl}_{3}\right) \delta 7.75(\mathrm{~d}, J=1.9 \mathrm{~Hz}$, $1 \mathrm{H}), 7.30(\mathrm{dd}, J=8.7,1.9 \mathrm{~Hz}, 1 \mathrm{H}), 7.19(\mathrm{~d}, J=8.6 \mathrm{~Hz}, 1 \mathrm{H}), 7.05(\mathrm{dd}, J=3.1,2.0 \mathrm{~Hz}, 1 \mathrm{H})$, $6.43(\mathrm{dd}, J=3.1,0.8 \mathrm{~Hz}, 1 \mathrm{H}), 3.77(\mathrm{~d}, J=138.4 \mathrm{~Hz}, 3 \mathrm{H}) ;{ }^{13} \mathrm{C} \mathrm{NMR}\left(100 \mathrm{MHz}, \mathrm{CDCl}_{3}\right) \delta$ 135.5, 130.2, 130.1 (d, $J=2.3 \mathrm{~Hz}), 124.5,123.4,112.8,110.8,100.7$ (d, J = 2.3 Hz), 33.14; GCMS: calc'd for $\mathrm{C}_{8}{ }^{13} \mathrm{CH}_{8} \mathrm{BrN}[\mathrm{M}]^{+}:$209.99, found: 210.00 . 


\section{General Procedure for One-pot Reaction of N-Alkylation of Indoles}

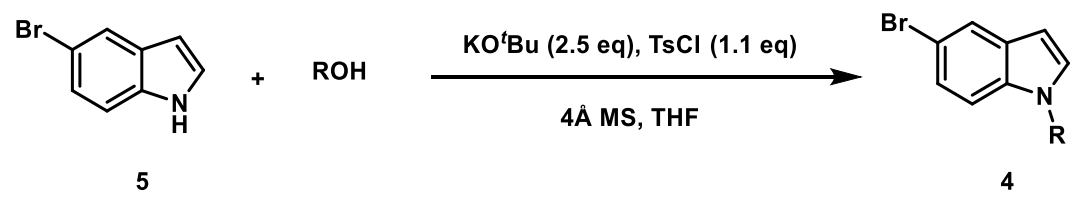

General procedure D: To a dry Schlenk tube equipped with a magnetic stirring bar, 5bromo- $1 H$-indole ( 1 equiv), $\mathrm{TsCl}$ (1.1 equiv), $\mathrm{ROH}$ ( 1.1 equiv), $\mathrm{KO}^{t} \mathrm{Bu}(2.5$ equiv), $4 \AA \mathrm{MS}$ and THF were added under an atmosphere of dry argon. Then tube was stirred at room temperature until the completion of the reaction as monitored by TLC. After finished, the reaction mixture was filtered through a short pad of Celite, then washed with EA (10 mL). Then the filtrate was concentrated under reduced pressure. The crude mixture was purified by silica gel chromatography to give the desired product.

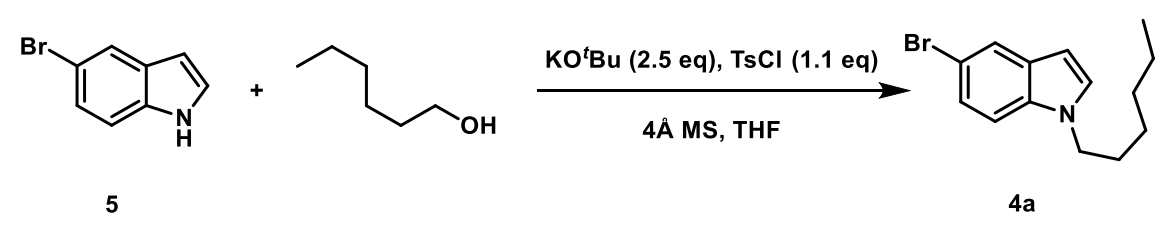

5-Bromo-1-hexyl-1H-indole (4a): The general procedure D was followed. The reaction was performed with 5-bromo-1H-indole 5 (98.0 mg, $0.5 \mathrm{mmol})$, TsCl (105.0 mg, $0.55 \mathrm{~mol})$, hexanol (61.2 mg, $0.6 \mathrm{mmol}), \mathrm{KO}^{t} \mathrm{Bu}(140.0 \mathrm{mg}, 1.25 \mathrm{mmol}), 4 \AA ̊ \mathrm{MS}(250 \mathrm{mg})$ in $5 \mathrm{~mL}$ THF. The desired product $4 \mathbf{a}$ (102.3 mg, 73\% yield) was obtained after purification by silica gel chromatography $\left(1 \% \mathrm{CH}_{2} \mathrm{Cl}_{2}\right.$ in $\left.\mathrm{PE}\right)$.

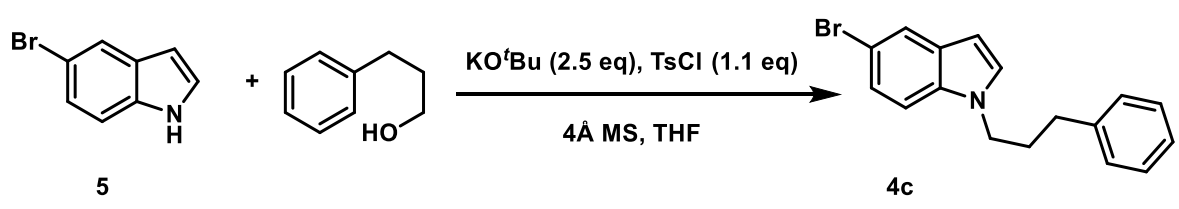

5-Bromo-1-(3-phenylpropyl)-1H-indole (4c): The general procedure D was followed. The reaction was performed with 5-bromo- $1 H$-indole 5 (39.3 mg, $0.2 \mathrm{mmol}$ ), 3phenylpropan-1-ol (30.0 mg, $0.22 \mathrm{mmol}), \mathrm{KO}^{\mathrm{t}} \mathrm{Bu}(58.3 \mathrm{mg}, 0.5 \mathrm{mmol})$ and $4 \AA \mathrm{MS}(200$ $\mathrm{mg})$ in $2 \mathrm{~mL}$ THF. The desired product $4 \mathrm{c}$ (31.4 mg, 50\% yield) was obtained after purification by silica gel chromatography (5\% PE in EA). 


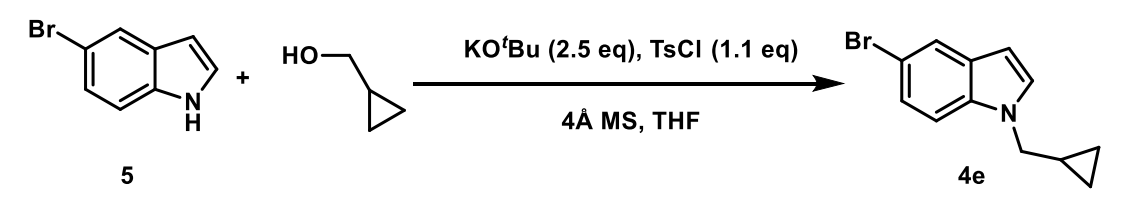

5-Bromo-1-(cyclopropylmethyl)-1H-indole(4e): The general procedure D was followed. The reaction was performed with 5-bromo- $1 H$-indole 5 (39.3 mg, $0.2 \mathrm{mmol})$, cyclopropylmethanol (15.8 mg, $0.22 \mathrm{mmol}), \mathrm{KO}^{t} \mathrm{Bu}(58.3 \mathrm{mg}, 0.5 \mathrm{mmol})$ and $4 \AA \mathrm{MS}(200$ $\mathrm{mg})$ in $2 \mathrm{~mL}$ THF. The desired product $4 \mathrm{e}$ (30.7 $\mathrm{mg}, 61 \%$ yield) was obtained after purification by silica gel chromatography (5\% PE in EA).

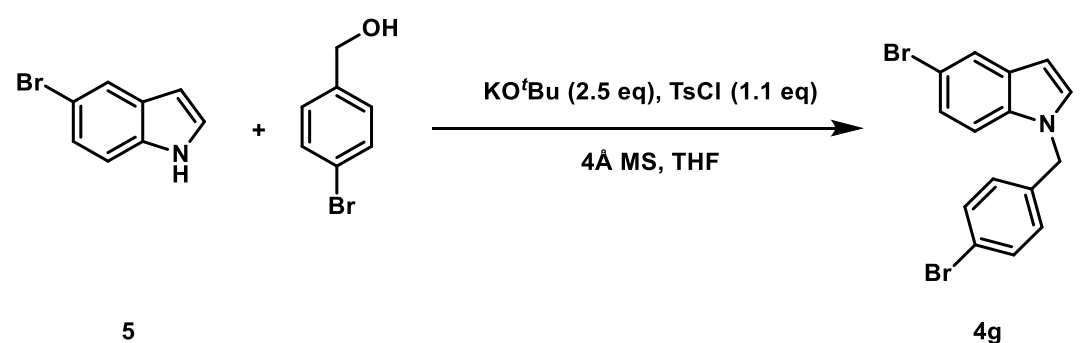

5-Bromo-1-(4-bromobenzyl)-1H-indole $(\mathbf{4 g})$ : The general procedure $\boldsymbol{D}$ was followed. The reaction was performed with 5-bromo- $1 H$-indole 5 (39.3 $\mathrm{mg}, 0.2 \mathrm{mmol})$, (4bromophenyl)methanol (41.1 mg, $0.22 \mathrm{mmol}), \mathrm{KO}^{t} \mathrm{Bu}(58.3 \mathrm{mg}, 0.5 \mathrm{mmol})$ and $4 \AA \mathrm{MS}$ (200 mg) in $2 \mathrm{~mL}$ THF. The desired product $\mathbf{4 g}$ (39.5 mg, 54\% yield) was obtained after purification by silica gel chromatography (5\% PE in EA).

\section{Synthesis of 8 (Pramoxine) and 8-d (Deuterated Pramoxine)}

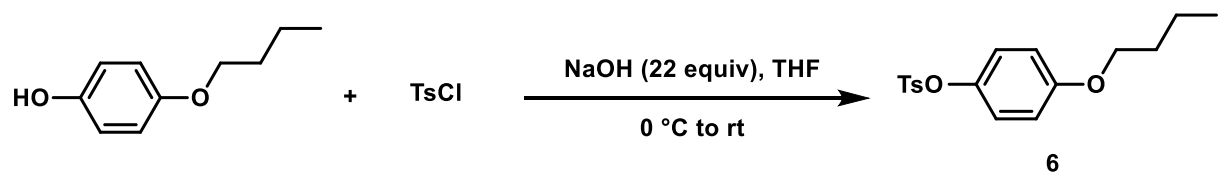

4-Butoxyphenyl 4-methylbenzenesulfonate (6): To a dry Schlenk flask equipped with a magnetic stirring bar, 4-butoxyphenol (0.16 g, $1 \mathrm{mmol}), \mathrm{NaOH}(0.88 \mathrm{~g}, 22 \mathrm{mmol})$ and 10 $\mathrm{mL}$ THF were added under an atmosphere of dry argon and stirred for 15 minutes at room temperature. Then $\mathrm{TsCl}(0.21 \mathrm{~g}, 1.1 \mathrm{mmol}, 1.1$ equiv, in $10 \mathrm{~mL} \mathrm{THF})$ was slowly added within $15 \mathrm{~min}$ at $0{ }^{\circ} \mathrm{C}$. The reaction mixture was stirred at room temperature for $12 \mathrm{~h}$, then quenched with $\mathrm{H}_{2} \mathrm{O}(20 \mathrm{~mL})$ and extracted with EA $(20 \mathrm{~mL} \times 3)$. The combined organic 
layers were washed with brine and dried over anhydrous $\mathrm{Na}_{2} \mathrm{SO}_{4}$. The solvents were removed under reduced pressure. The desired product $6(0.32 \mathrm{~g}, 99 \%$ yield $)$ was obtained after purification by silica gel chromatography (4\% EA in PE) to get the corresponding product. ${ }^{1} \mathrm{H}$ NMR $\left(400 \mathrm{MHz}, \mathrm{CDCl}_{3}\right) \delta 7.68(\mathrm{~d}, J=8.3 \mathrm{~Hz}, 2 \mathrm{H}), 7.30(\mathrm{~d}, J=8.1 \mathrm{~Hz}, 2 \mathrm{H})$, $6.85(\mathrm{~d}, J=9.1 \mathrm{~Hz}, 2 \mathrm{H}), 6.75(\mathrm{~d}, J=9.1 \mathrm{~Hz}, 2 \mathrm{H}), 3.89(\mathrm{t}, J=6.5 \mathrm{~Hz}, 2 \mathrm{H}), 2.44(\mathrm{~s}, 3 \mathrm{H})$, $1.85-1.63(\mathrm{~m}, 2 \mathrm{H}), 1.52-1.34(\mathrm{~m}, 2 \mathrm{H}), 0.96(\mathrm{t}, J=7.4 \mathrm{~Hz}, 3 \mathrm{H}) ;{ }^{13} \mathrm{C} \mathrm{NMR}(100 \mathrm{MHz}$, $\left.\mathrm{CDCl}_{3}\right) \delta 157.9,145.3,143.0,132.4,129.8,128.7,123.4,115.1,68.2,31.4,21.9,19.3,14.0$; HRMS (ESI+) calc'd for $\mathrm{C}_{17} \mathrm{H}_{20} \mathrm{SO}_{4} \mathrm{~K}[\mathrm{M}+\mathrm{K}]^{+}:$359.0714, found: 359.0717.

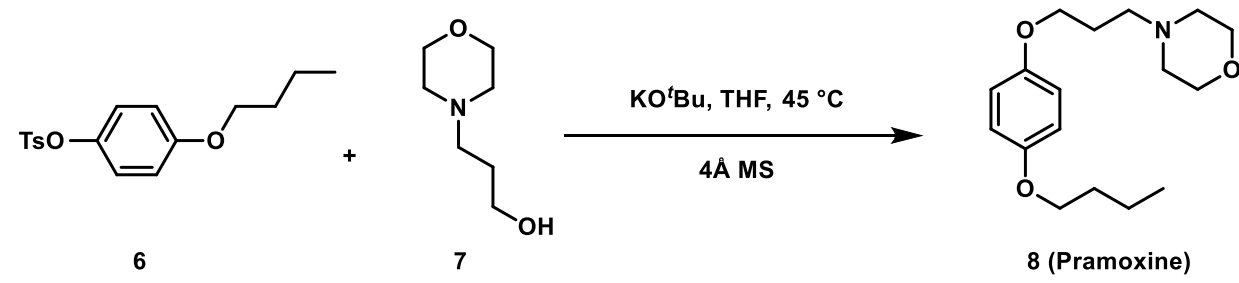

4-(3-(4-Butoxyphenoxy)propyl)morpholine (8): The general procedure $\mathrm{C}$ was followed. The reaction was performed with 4-butoxyphenyl 4-methylbenzenesulfonate 6 (64.1 mg, $0.2 \mathrm{mmol}$ ), 3-morpholinopropan-1-ol 7 (31.9 mg, $0.22 \mathrm{mmol}$ ), $\mathrm{KO}^{t} \mathrm{Bu}$ (26.9 mg, $0.24 \mathrm{mmol}$ ) and $4 \AA$ MS (200.0 mg) in $2 \mathrm{~mL}$ THF for $12 \mathrm{~h}$. The desired product 8 (34.3 mg, $62 \%$ yield) was obtained after purification by silica gel chromatography (5\% methanol in DCM). ${ }^{1} \mathrm{H}$ NMR (600 MHz, $\left.\mathrm{CDCl}_{3}\right) \delta 6.82(\mathrm{~s}, 4 \mathrm{H}), 3.96(\mathrm{t}, J=6.3 \mathrm{~Hz}, 2 \mathrm{H}), 3.90(\mathrm{t}, J=6.5 \mathrm{~Hz}, 2 \mathrm{H})$, $3.74-3.65(\mathrm{~m}, 4 \mathrm{H}), 2.56-2.43(\mathrm{~m}, 6 \mathrm{H}), 1.98-1.88(\mathrm{~m}, 2 \mathrm{H}), 1.79-1.69(\mathrm{~m}, 2 \mathrm{H}), 1.54-$ $1.40(\mathrm{~m}, 2 \mathrm{H}), 0.96(\mathrm{t}, J=6.9 \mathrm{~Hz}, 3 \mathrm{H})$. These spectroscopic data were consistent with those reported in the literature. ${ }^{21}$

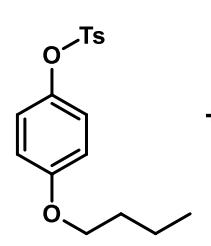

6

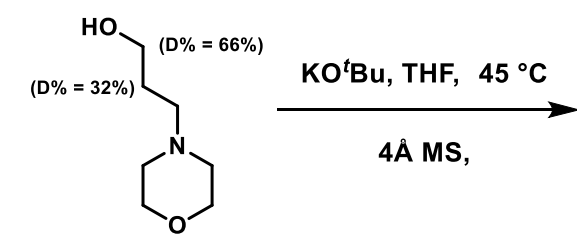

7-d

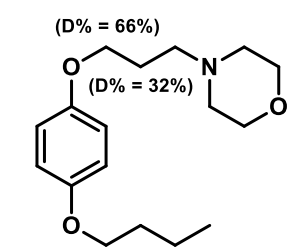

8-d (Deuterated Pramoxine)

4-(3-(4-Butoxyphenoxy)propyl-2,3,3-d3)morpholine (8-d): The general procedure $\mathrm{C}$ was followed. The reaction was performed with 4-butoxyphenyl 4- methylbenzenesulfonate 6 
(48.1 mg, $0.15 \mathrm{mmol}), 3$-morpholinopropan-1,1,2- $d_{3}-1$-ol 7-d (23.5 mg, $\left.0.16 \mathrm{mmol}\right)$, $\mathrm{KO}^{t} \mathrm{Bu}(20.2 \mathrm{mg}, 0.18 \mathrm{mmol})$ and $4 \AA \mathrm{MS}(100.0 \mathrm{mg})$ in $2 \mathrm{~mL}$ THF for $12 \mathrm{~h}$. The desired product 8-d (33.9 $\mathrm{mg}, 76 \%$ yield) was obtained after purification by silica gel chromatography (rinsed the silica gel with $0.2 \% \mathrm{Et}_{3} \mathrm{~N}$ in $\mathrm{PE}$, then with $25 \% \mathrm{EA}$ in PE as the eluent). ${ }^{1} \mathrm{H}$ NMR (400 MHz, $\left.\mathrm{CDCl}_{3}\right) \delta 6.82(\mathrm{~s}, 4 \mathrm{H}), 3.96$ (t, $\left.J=6.0 \mathrm{~Hz}, 0.69 \mathrm{H}\right), 3.90$ (t, $J=6.5 \mathrm{~Hz}, 2 \mathrm{H}), 3.73(\mathrm{t}, J=4.6 \mathrm{~Hz}, 4 \mathrm{H}), 2.58-2.40(\mathrm{~m}, 6 \mathrm{H}), 1.98-1.88(\mathrm{~m}, 1.39 \mathrm{H}), 1.77$ $-1.68(\mathrm{~m}, 2 \mathrm{H}), 1.53-1.41(\mathrm{~m}, 2 \mathrm{H}), 0.96(\mathrm{t}, J=7.4 \mathrm{~Hz}, 3 \mathrm{H}) ;{ }^{13} \mathrm{C} \mathrm{NMR}\left(100 \mathrm{MHz}, \mathrm{CDCl}_{3}\right)$ $\delta 153.4,153.1,115.5,68.4,67.1,55.7,53.9,31.6,26.4,19.4,14.0 ;$ HRMS (ESI+) calc'd for $\mathrm{C}_{17} \mathrm{H}_{25} \mathrm{D}_{3} \mathrm{NO}_{3}[\mathrm{M}+\mathrm{H}]^{+}:$297.2252, found: 297.2245.

\section{References}

(1) Ogiwara, Y.; Sakurai, Y.; Hattori, H.; Sakai, N. Org. Lett. 2018, $20,4204$.

(2) Tolnai, G. L.; Ganss, S.; Brand, J. P.; Waser, J. Org. Lett. 2013, 15, 112.

(3) Zheng, H.-X.; Shan, X.-H.; Qu, J.-P.; Kang, Y.-B. Org. Lett. 2017, 19, 5114.

(4) Kitano, M.; Kojima, A.; Nakano, K.; Miyagishi, A.; Noguchi, T.; Ohashi, N. Chem. Pharm. Bull. 1999, 47, 1538.

(5) Kong, A.; Han, X.; Lu, X. Org. Lett. 2006, 8, 1339.

(6) Jiang, X.; Yang, J.; Zhang, F.; Yu, P.; Yi, P.; Sun, Y.; Wang, Y. Org. Lett. 2016, 18, 3154.

(7) Klare, H. F. T.; Oestreich, M.; Ito, J.; Nishiyama, H.; Ohki, Y.; Tatsumi, K. J. Am. Chem. Soc. 2011, $133,3312$.

(8) Taylor, N. J.; Emer, E.; Preshlock, S.; Schedler, M.; Tredwell, M.; Verhoog, S.; Mercier, J.; Genicot, C.; Gouverneur, V. J. Am. Chem. Soc. 2017, 139, 8267.

(9) Xi, X.; Chen, T.; Zhang, J.-S.; Han, L.-B. Chem. Comm. 2018, 54, 1521.

(10) Wang, D.-Y.; Yang, Z.-K.; Wang, C.; Zhang, A.; Uchiyama, M. Angew. Chem. Int. Ed. 2018, 57, 3641 .

(11) Shen, Z.; Zhang, S.; Geng, H.; Wang, J.; Zhang, X.; Zhou, A.; Yao, C.; Chen, X.; Wang, W. Org. Lett. 2019, 21, 448.

(12) Alonso, F.; Yus, M. Tetrahedron 1992, 48, 2709.

(13) Guo, W.-J.; Wang, Z. -X. J. Org. Chem. 2013, 78, 1054.

(14) Rovira, M.; Soler, M.; Güell, I.; Wang, M-Z.; Gómez, L.; Ribas, X. J. Org. Chem. 2016, 78, 1054.

(15) Maraš, N.; Polanc, S.; Kočevar, M. Tetrahedron 2008, 64, 11618.

(16) Wongma, K.; Bunbamrung, N.; Thongpanchang, T. Tetrahedron 2016, 72, 1533.

(17) Chen, S.; Lu, G.; Ca, C. New J. Chem. 2015, 39, 5360.

(18) Babu, D. D.; Su, R.; El-Shafei, A.; Adhikari, A. V. Electrochimica. Acta. 2016, 198, 10.

(19) Li, M.; Kang, S.; Du, J.; Zhang, J.; Wang, J.; Ariga, K. Angew. Chem. Int. Ed. 2018, 57, 4936.

(20) Lei, X.; Jalla, A.; Shama, M. A. A.; Stafford, J. M.; Cao, B. Synthesis 2015, 47, 2578.

(21) Guo, Y.; Fan, X.-M.; Nie, M.; Liu, H.-W.; Liao, D.-H.; Pan, X.-D.; Ji, Y.-F. Eur. J. Org. Chem. 2015, 4744. 


\section{GC-MS Spectra}
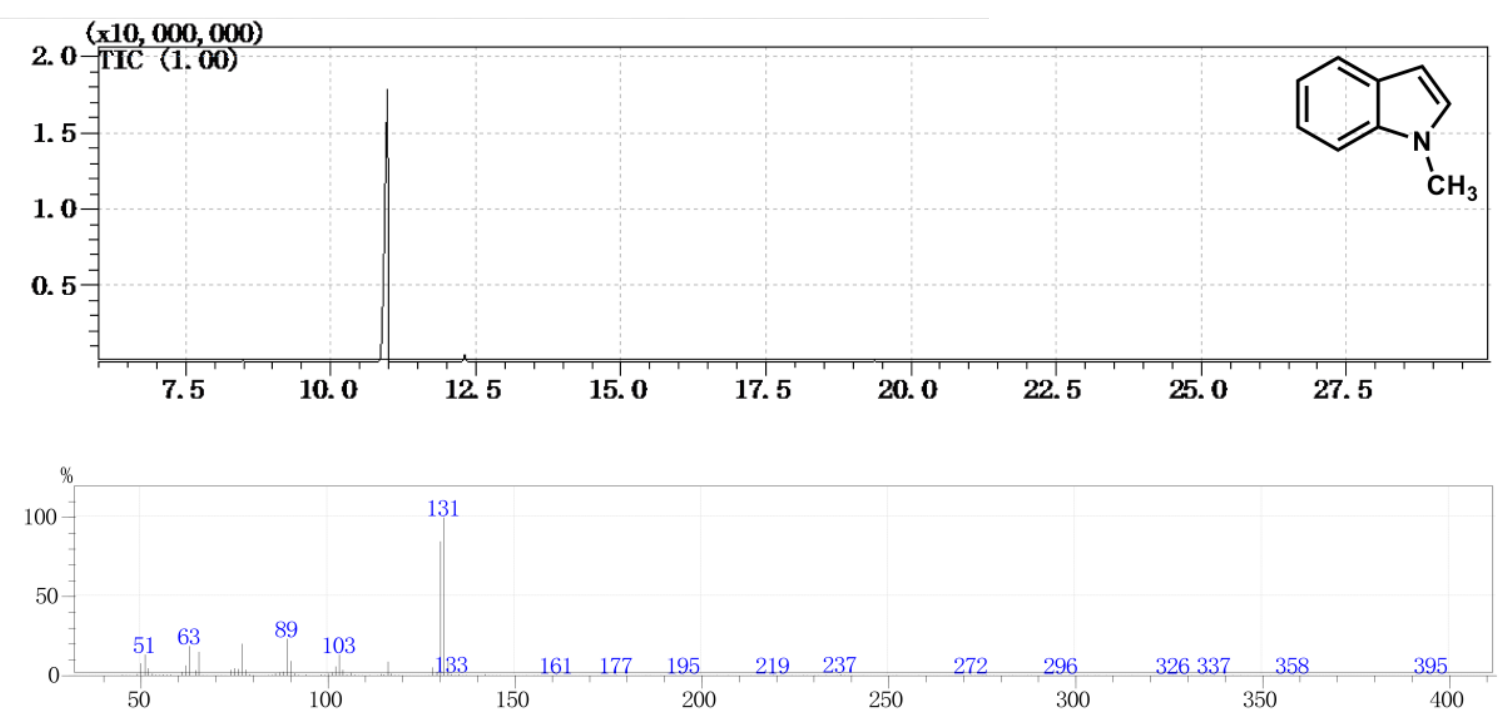

GC-MS of $\mathbf{2 a}$
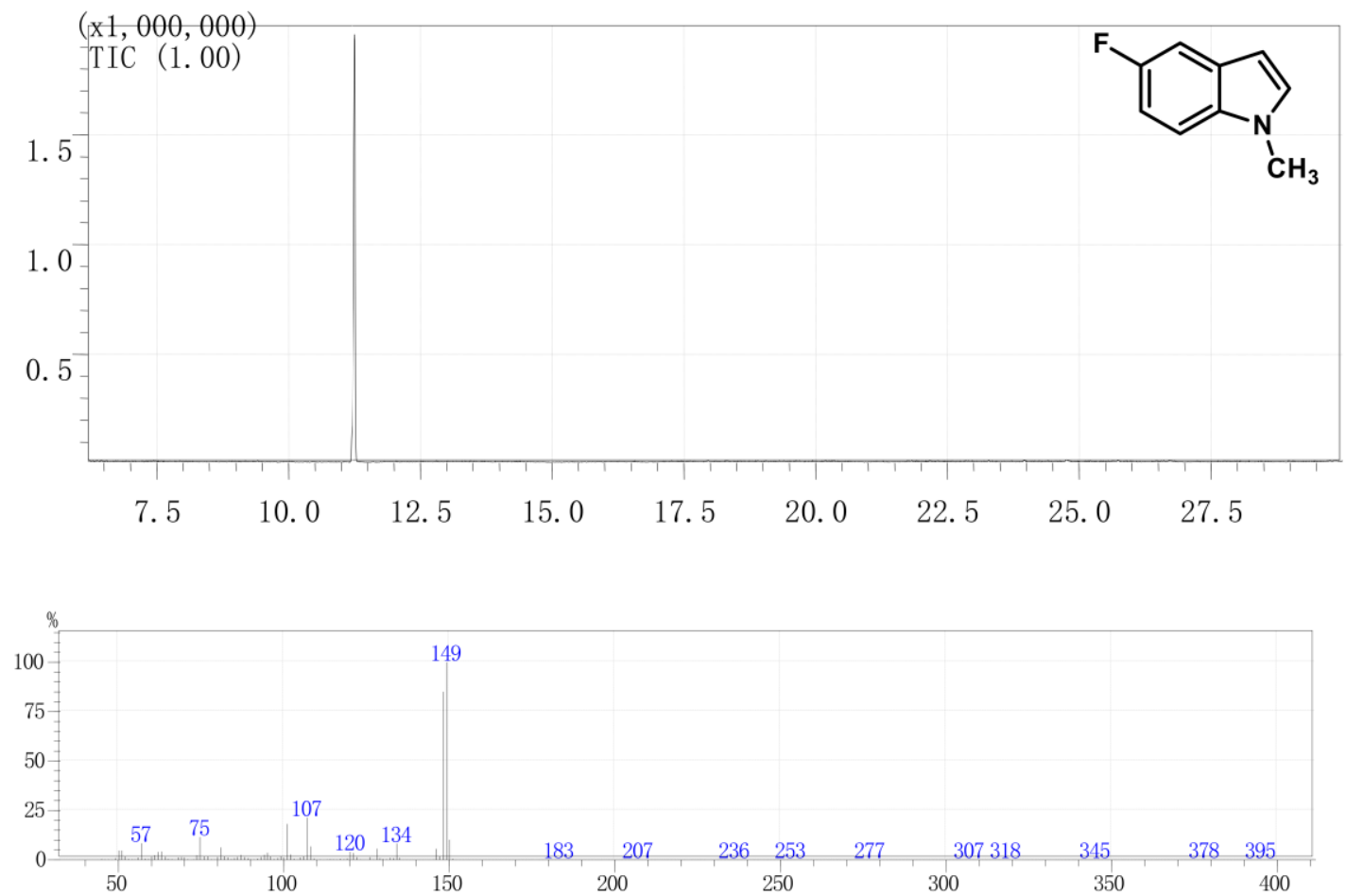

GC-MS of $\mathbf{2 b}$ 

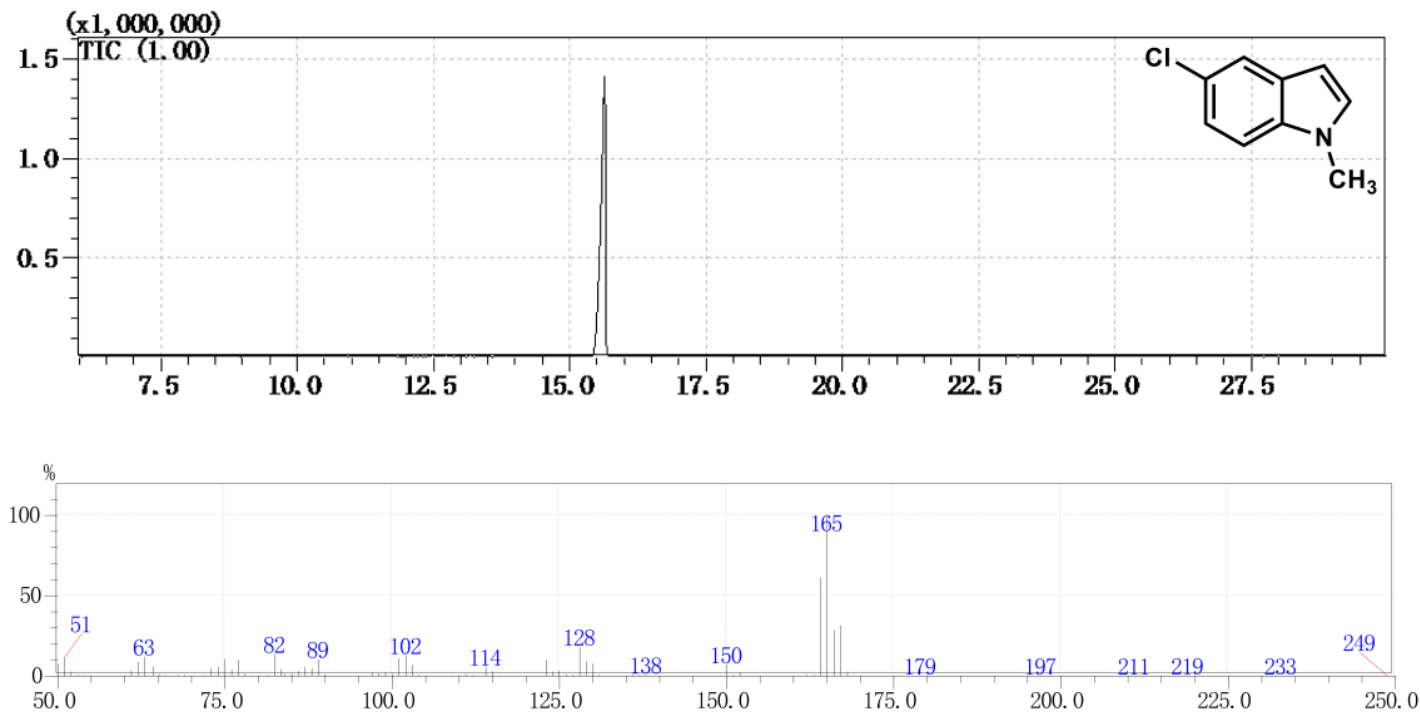

GC-MS of $2 c$
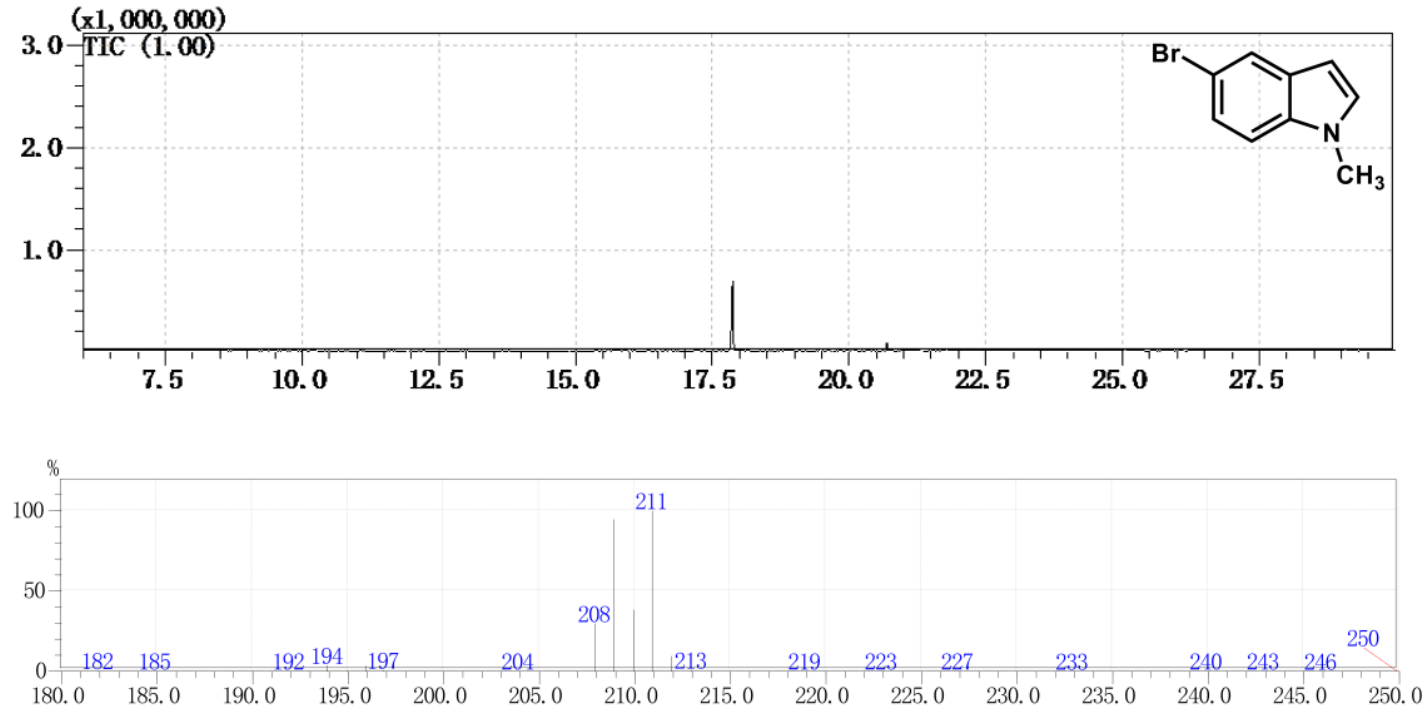

GC-MS of 2d 

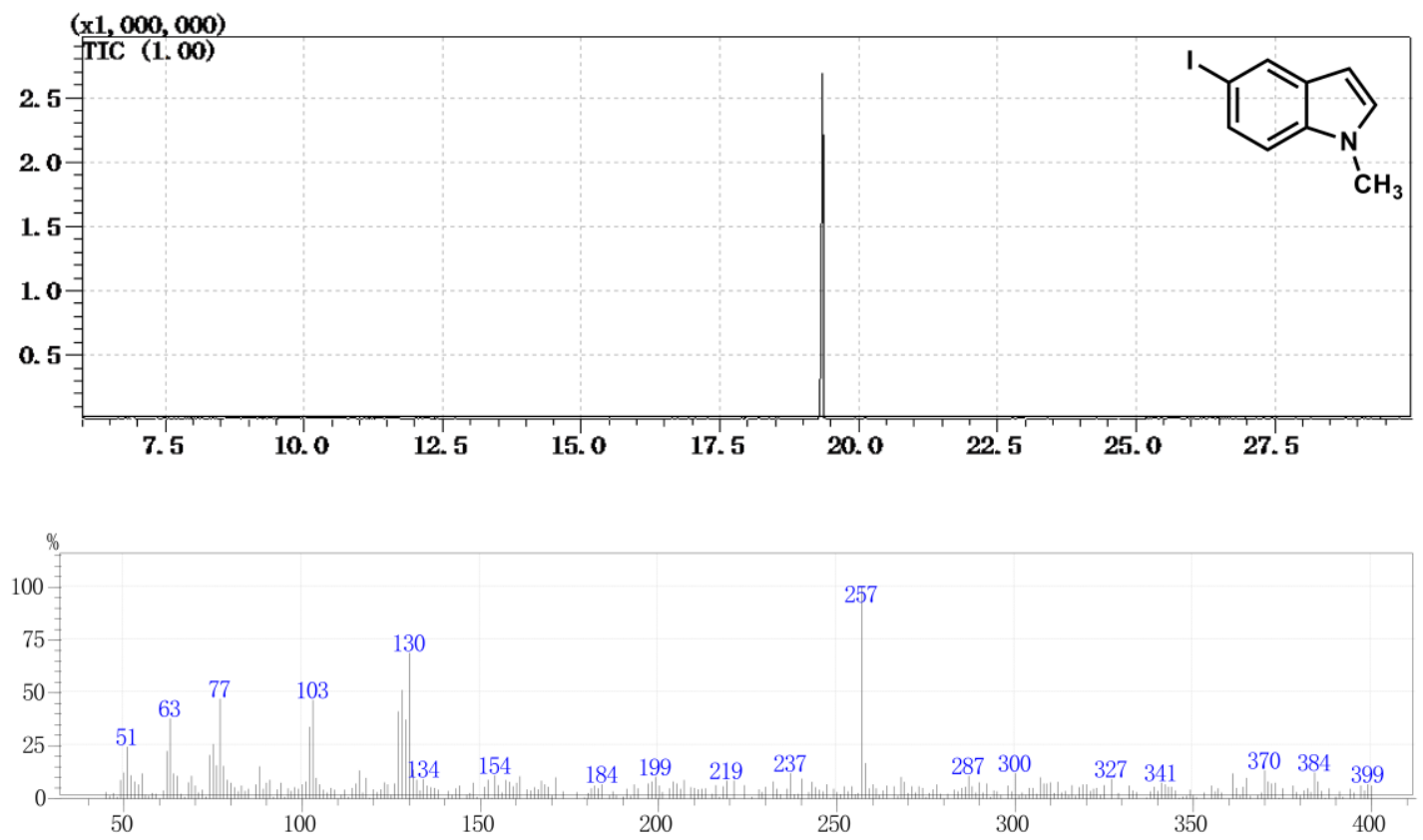

GC-MS of $\mathbf{2 e}$
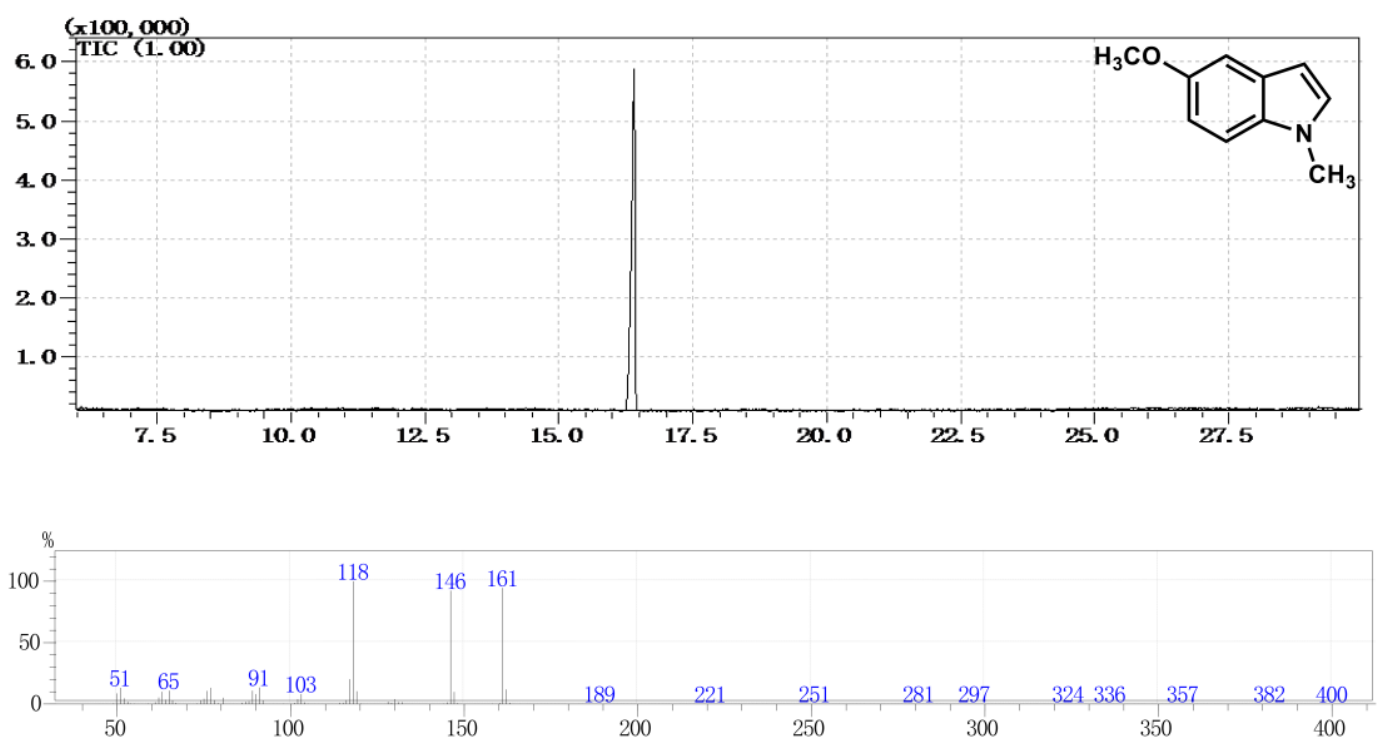

GC-MS of $\mathbf{2 f}$ 

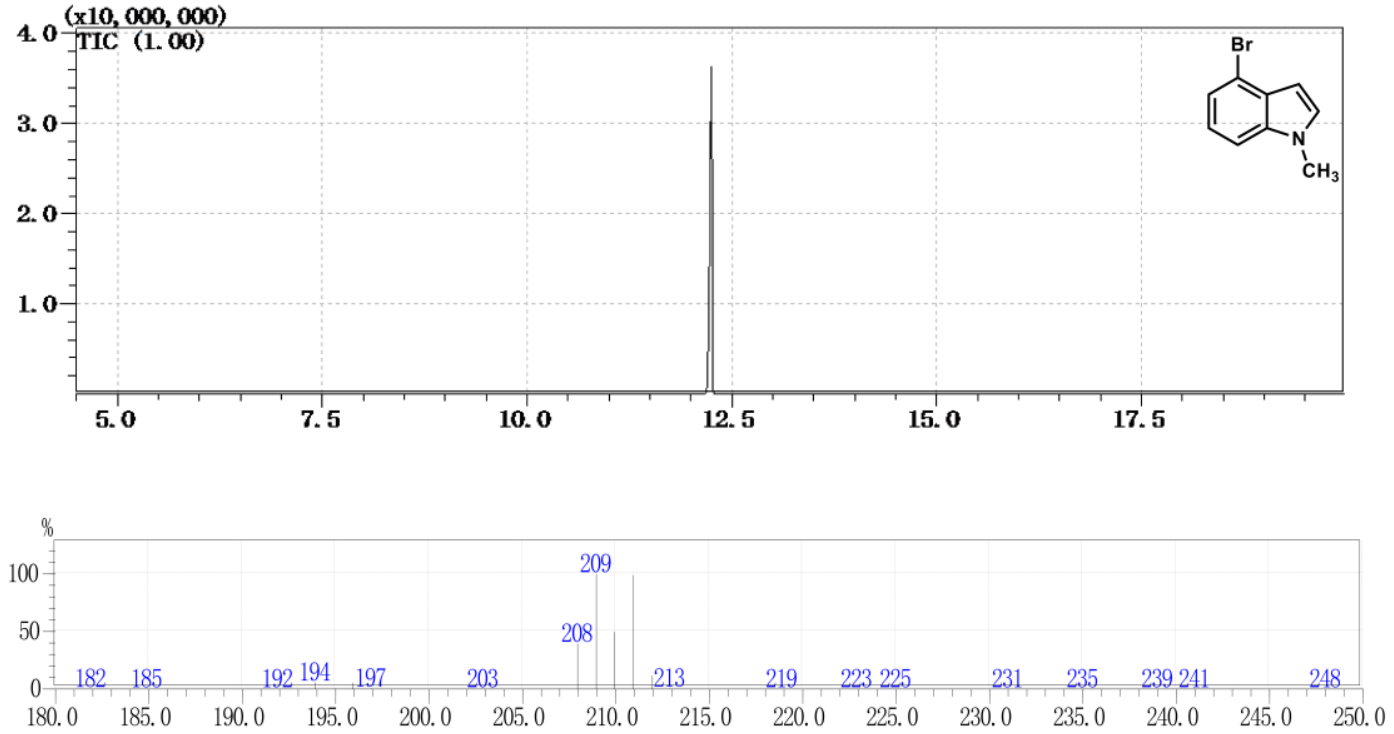

GC-MS of $\mathbf{2 g}$
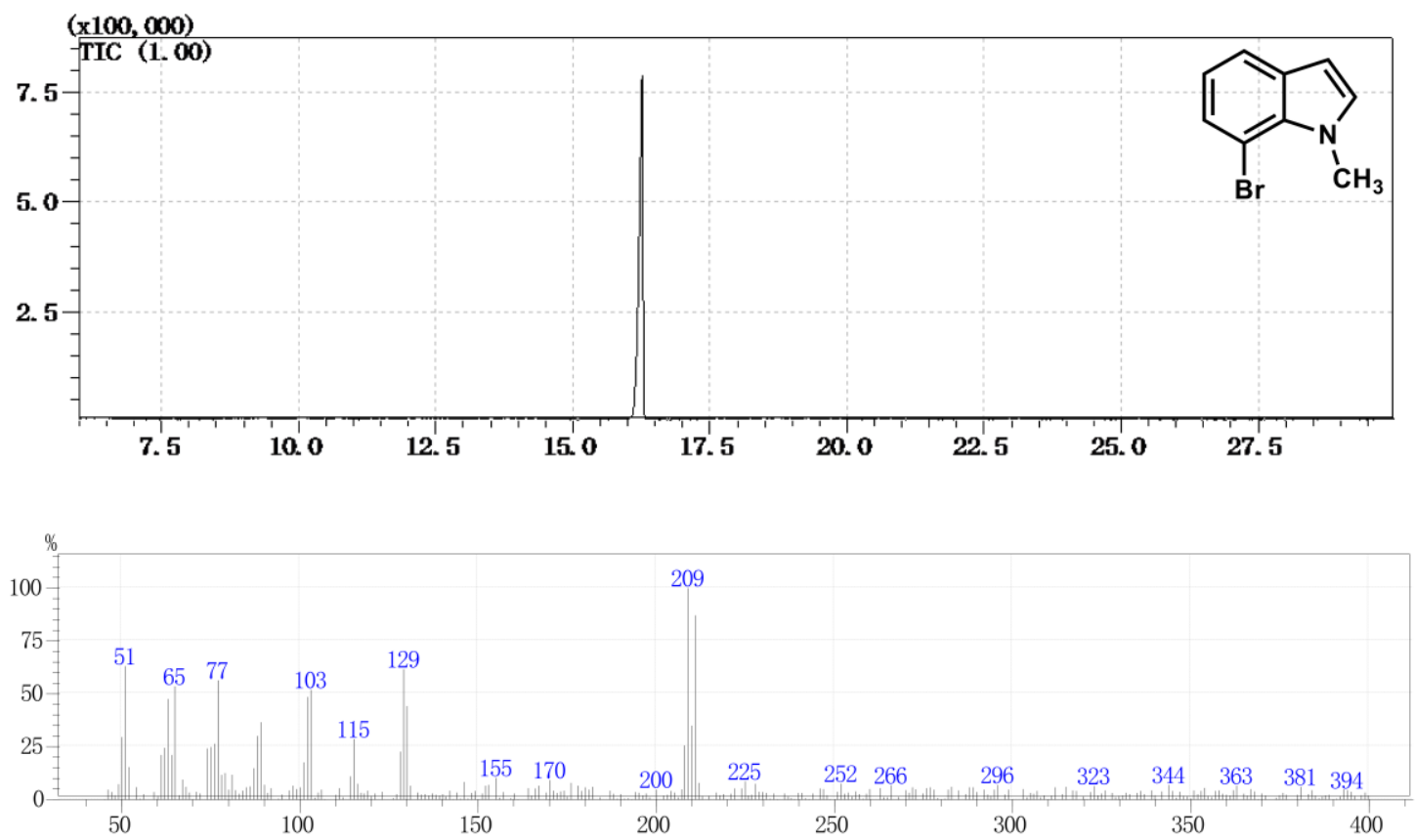

GC-MS of $\mathbf{2 h}$ 

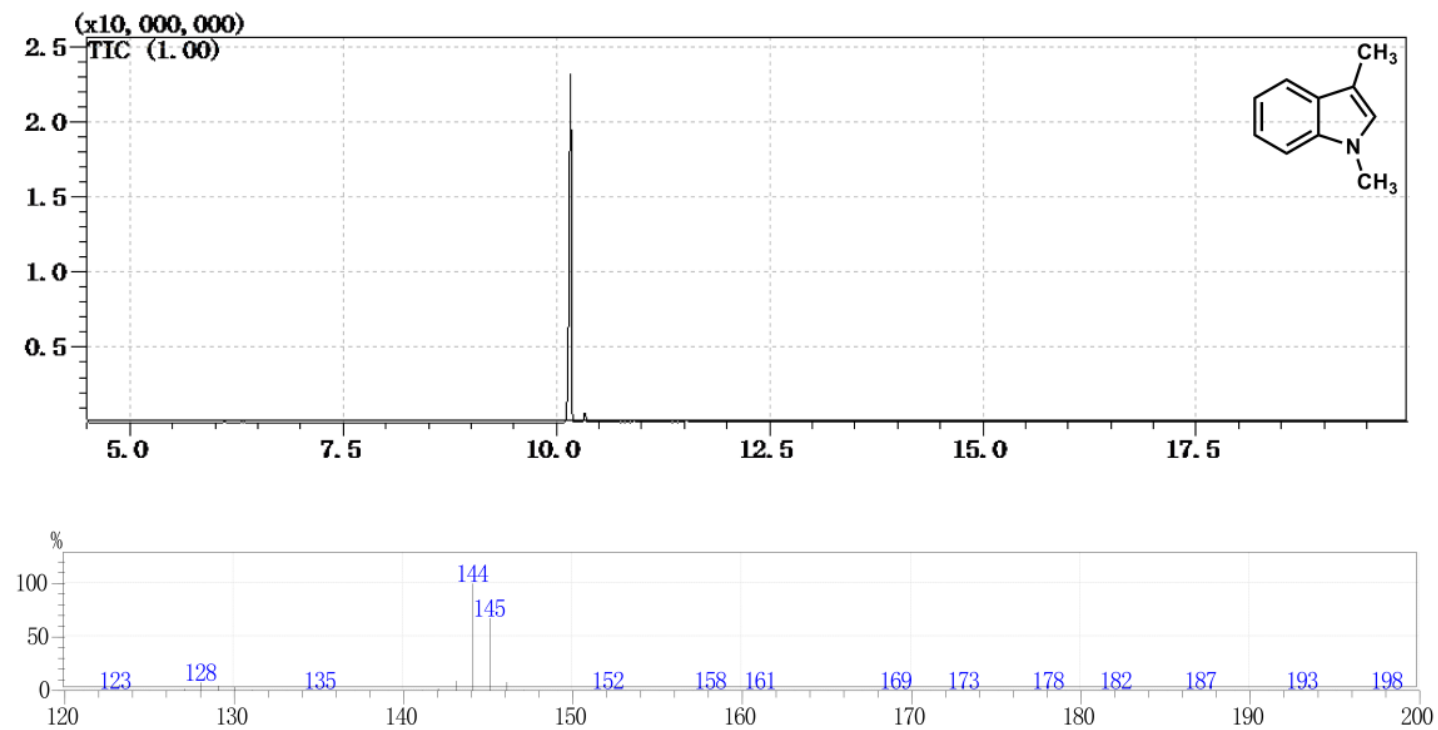

GC-MS of $\mathbf{2 k}$
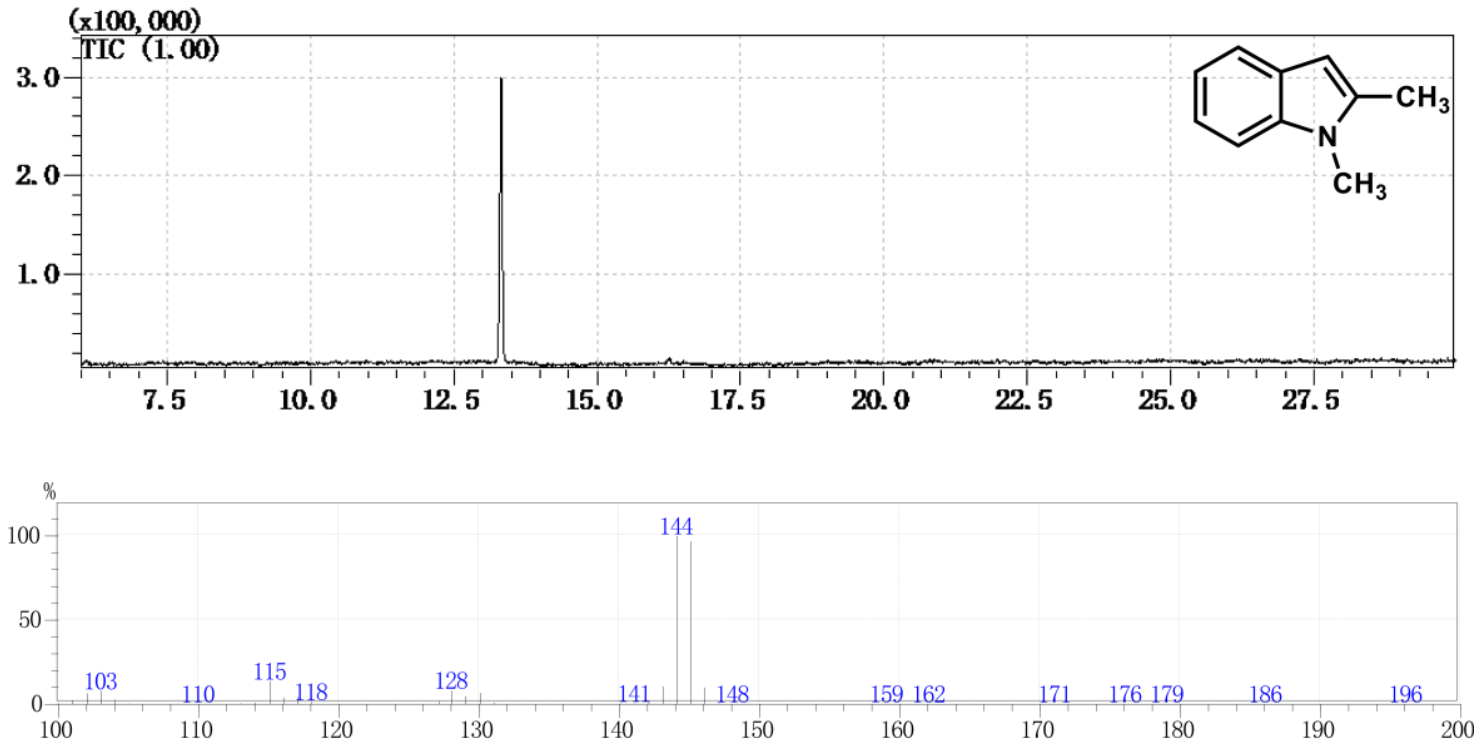

GC-MS of 2l 

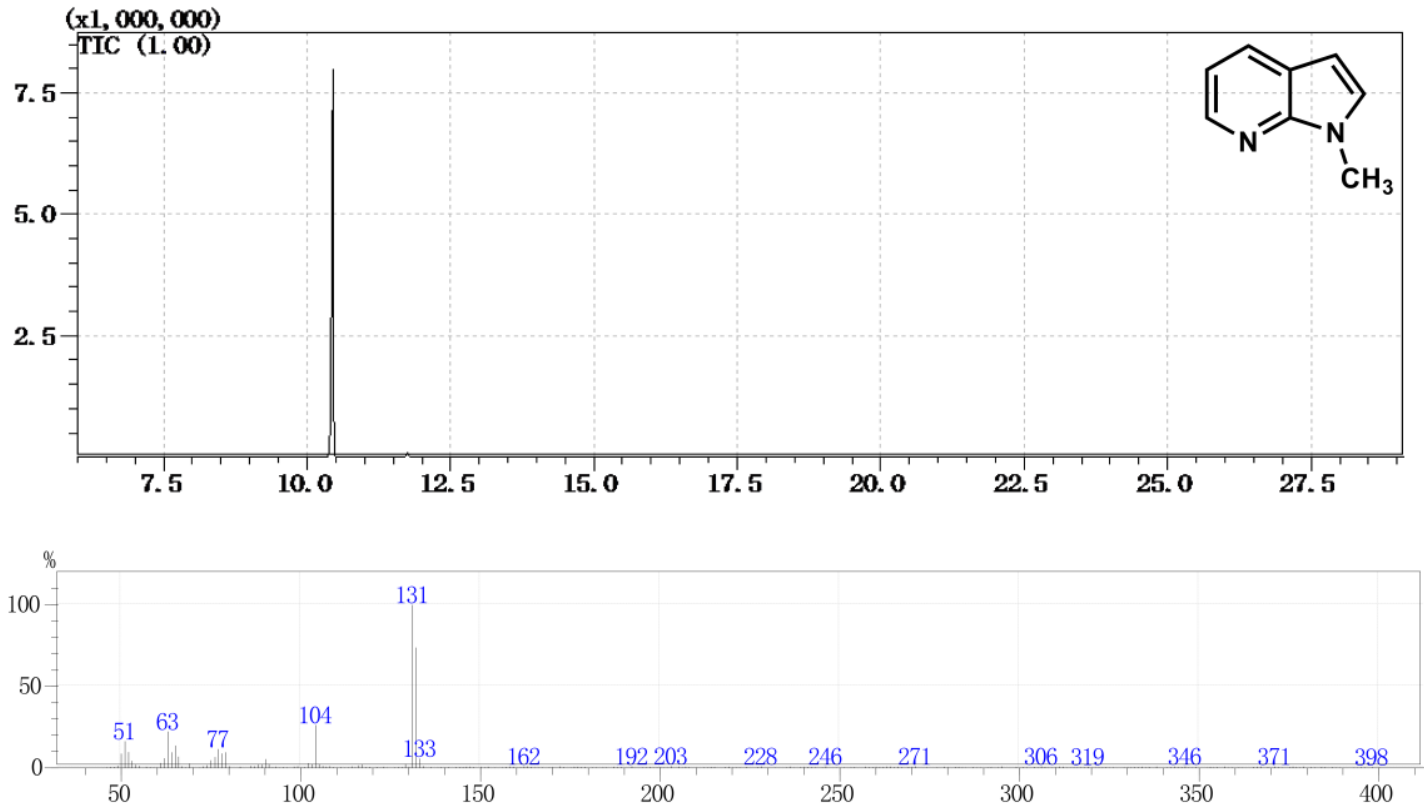

GC-MS of $\mathbf{2 m}$ 


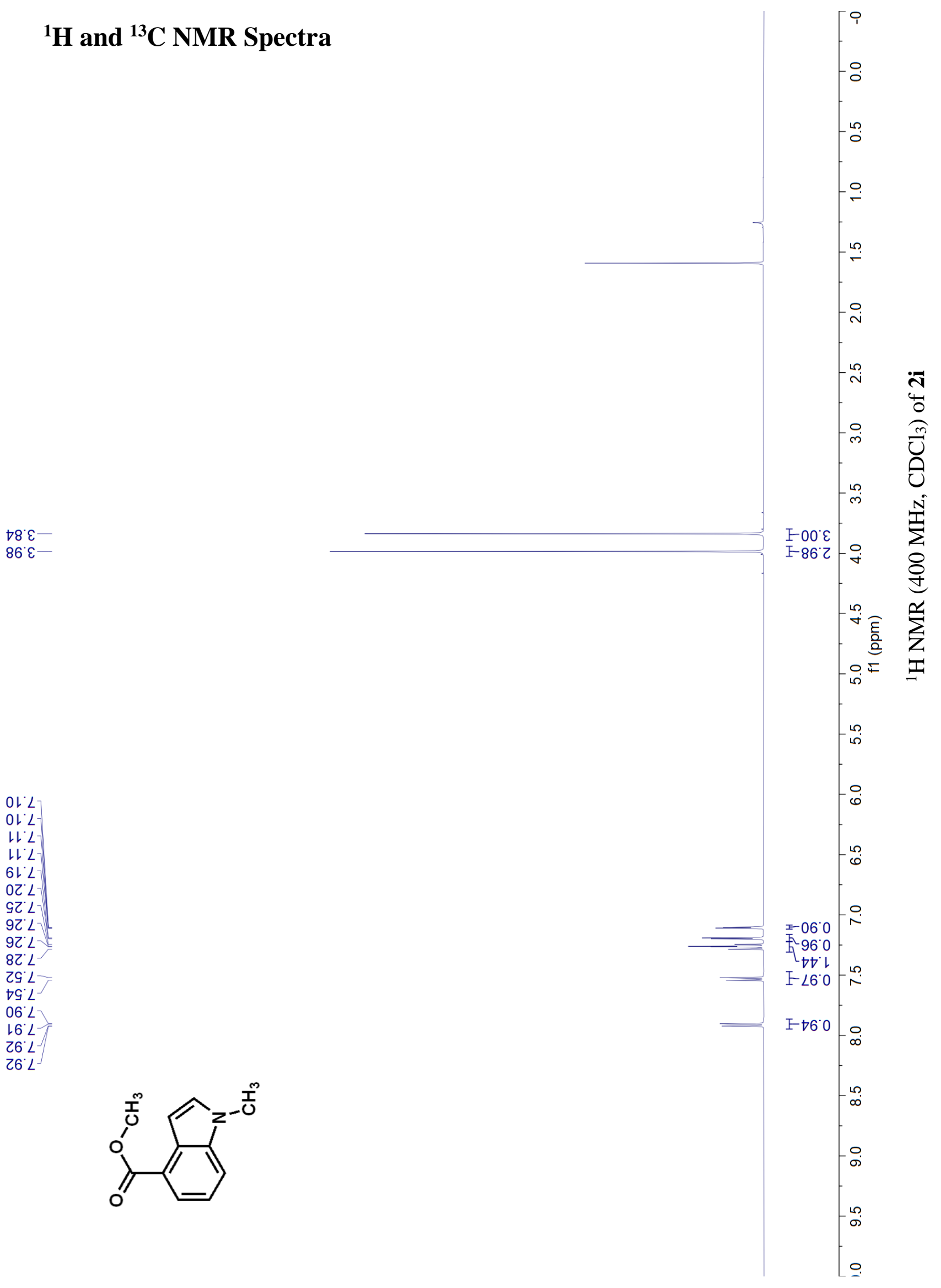




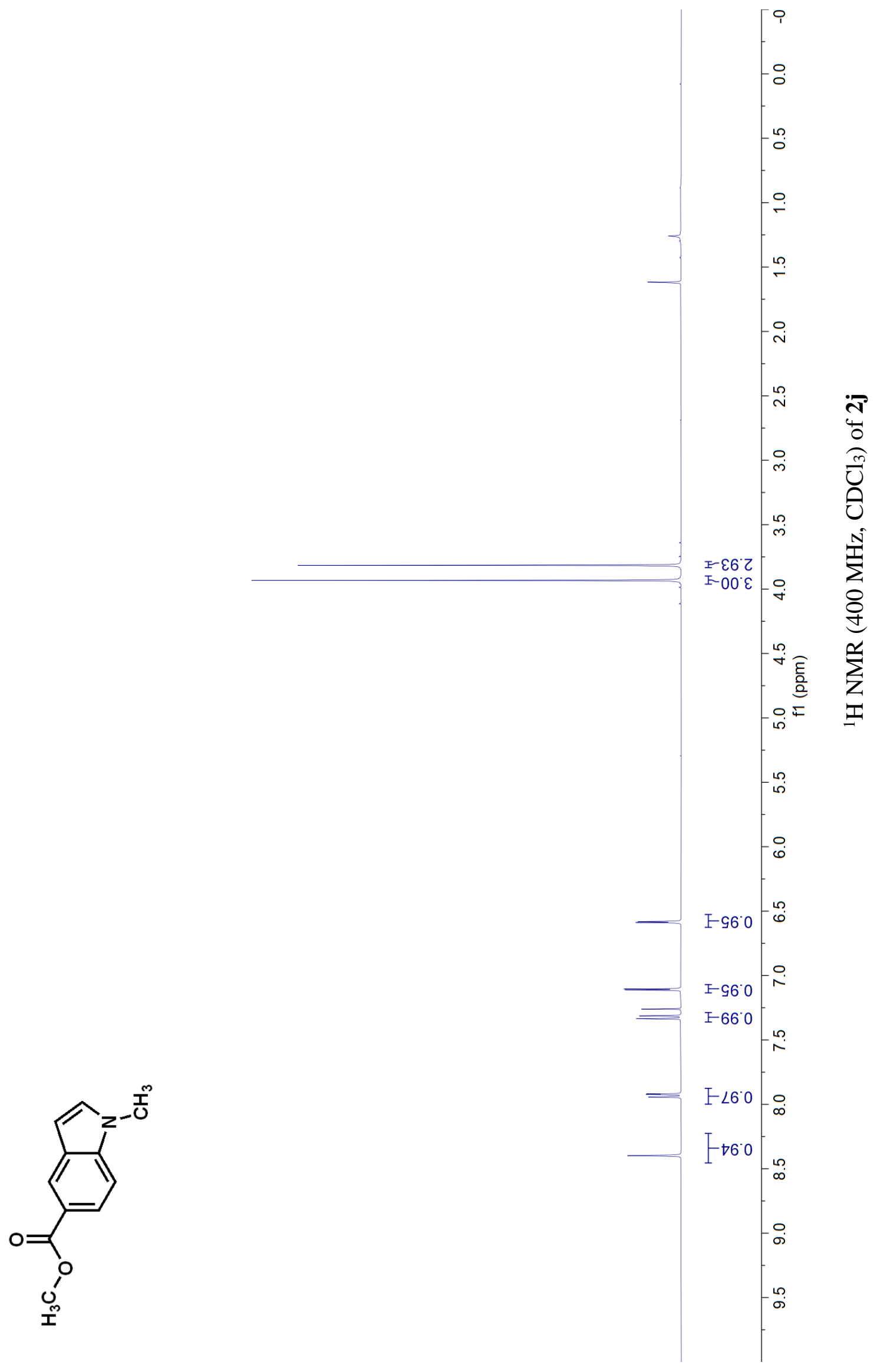

$69^{\circ} 9$

$O L L$

UL

9Z $L$

IE $L$

$\nabla \varepsilon L$

$26^{\circ} \mathrm{L}$

$26^{\circ} \mathrm{L}=$

$96^{\circ} \mathrm{L}$

$0 \nabla^{\circ} 8$

$07^{\circ}$ 


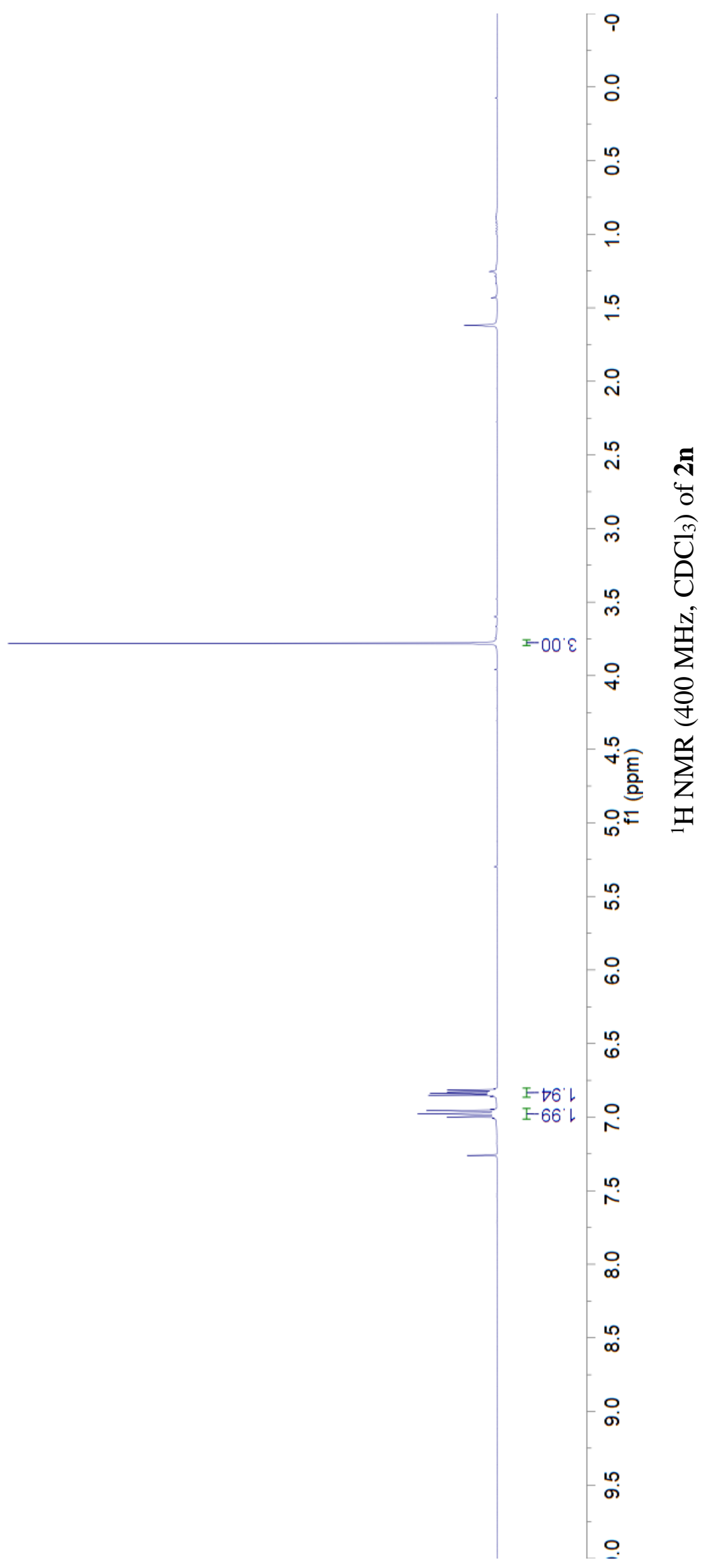




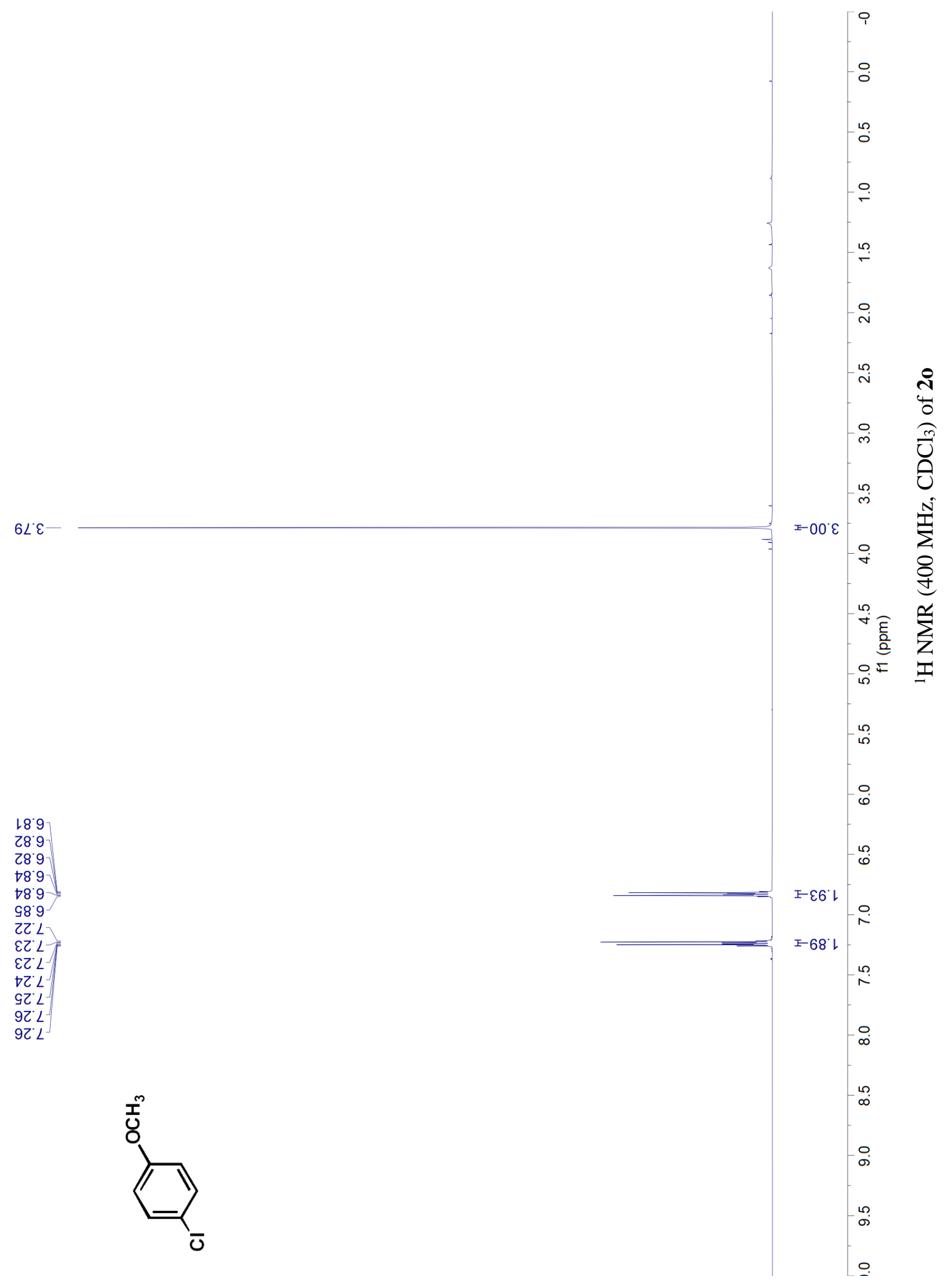




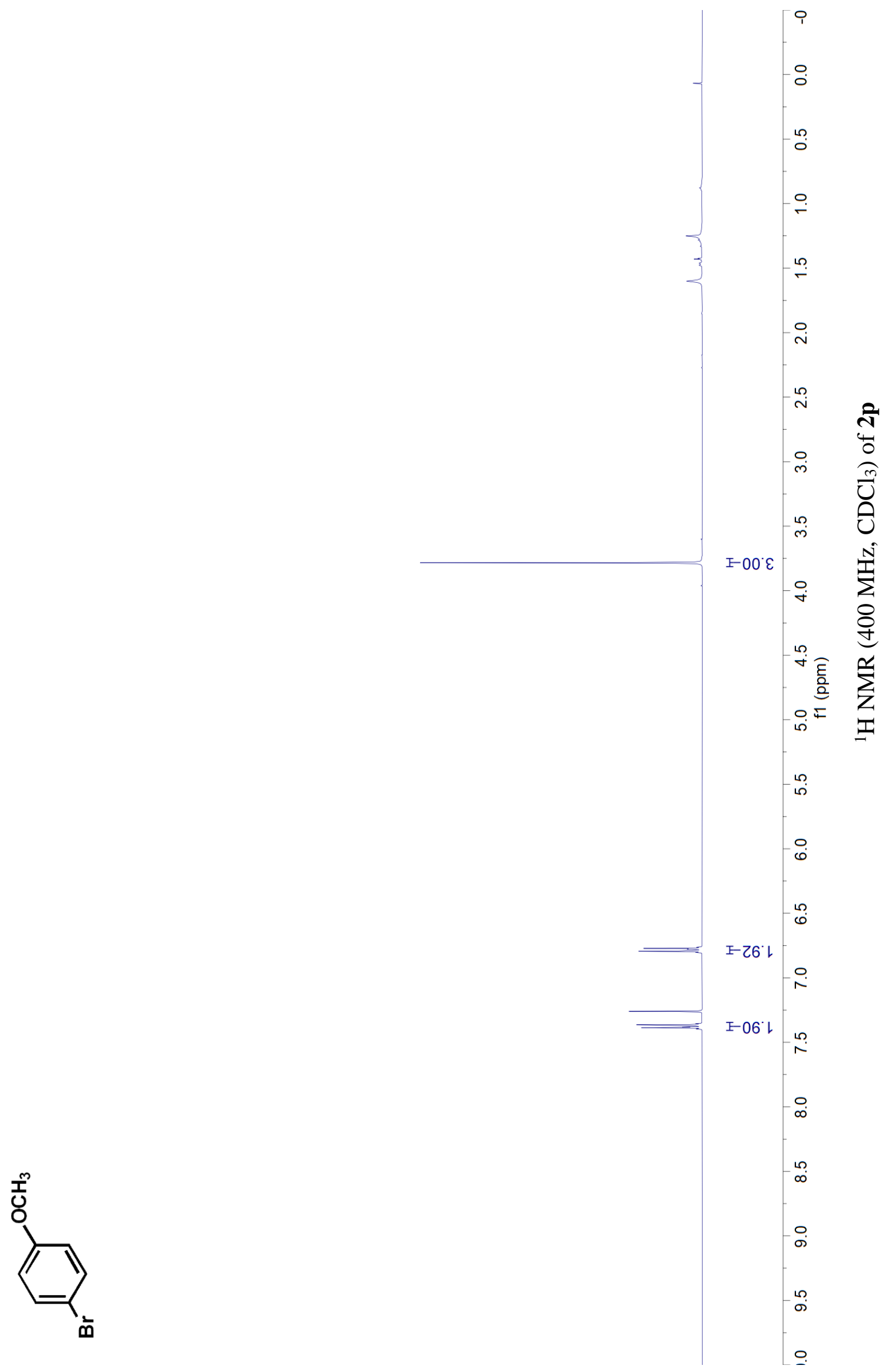




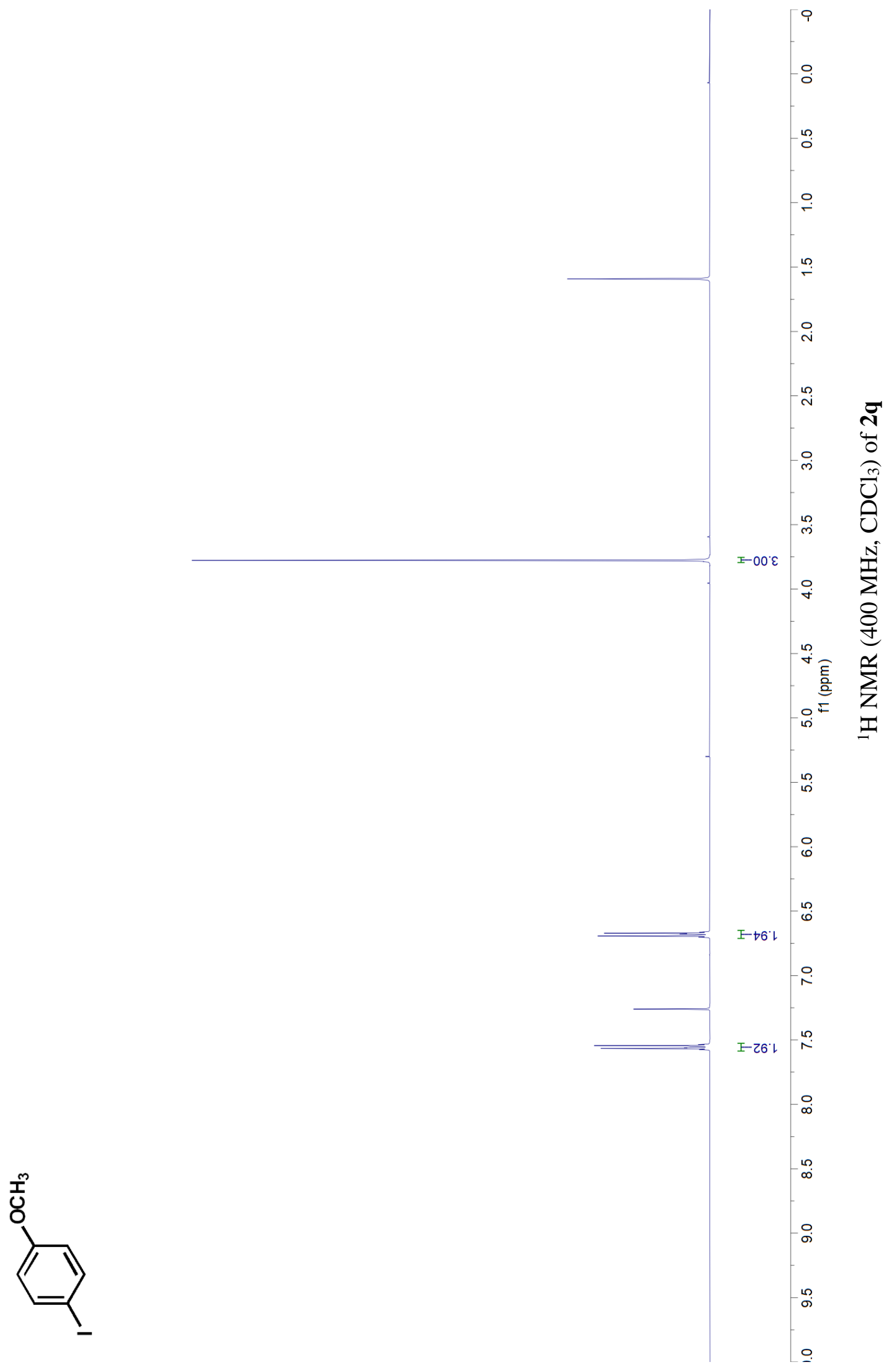


6レて

$9 \mathbf{Z}^{\circ}$

$08^{\circ} \varepsilon-$

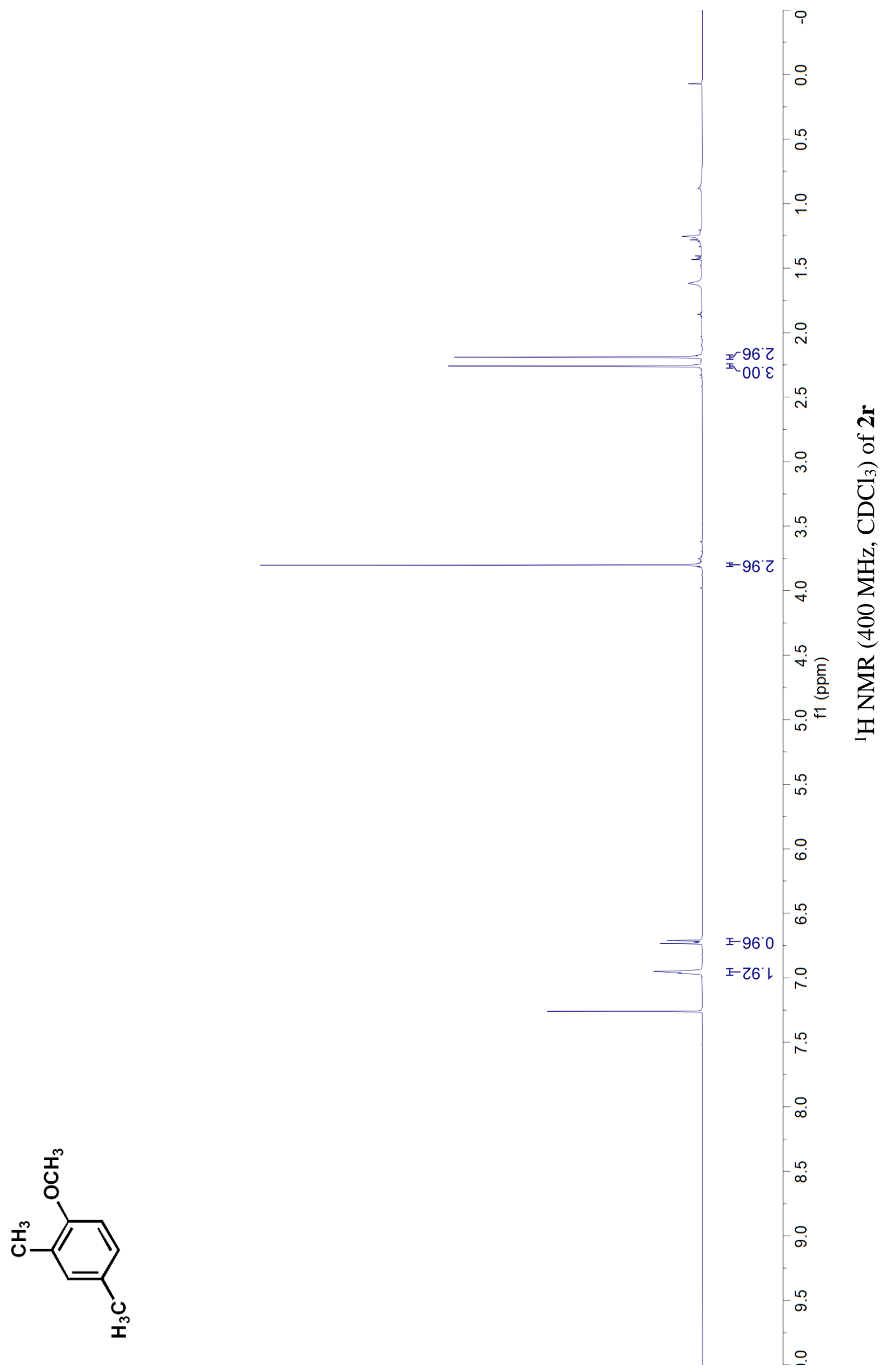




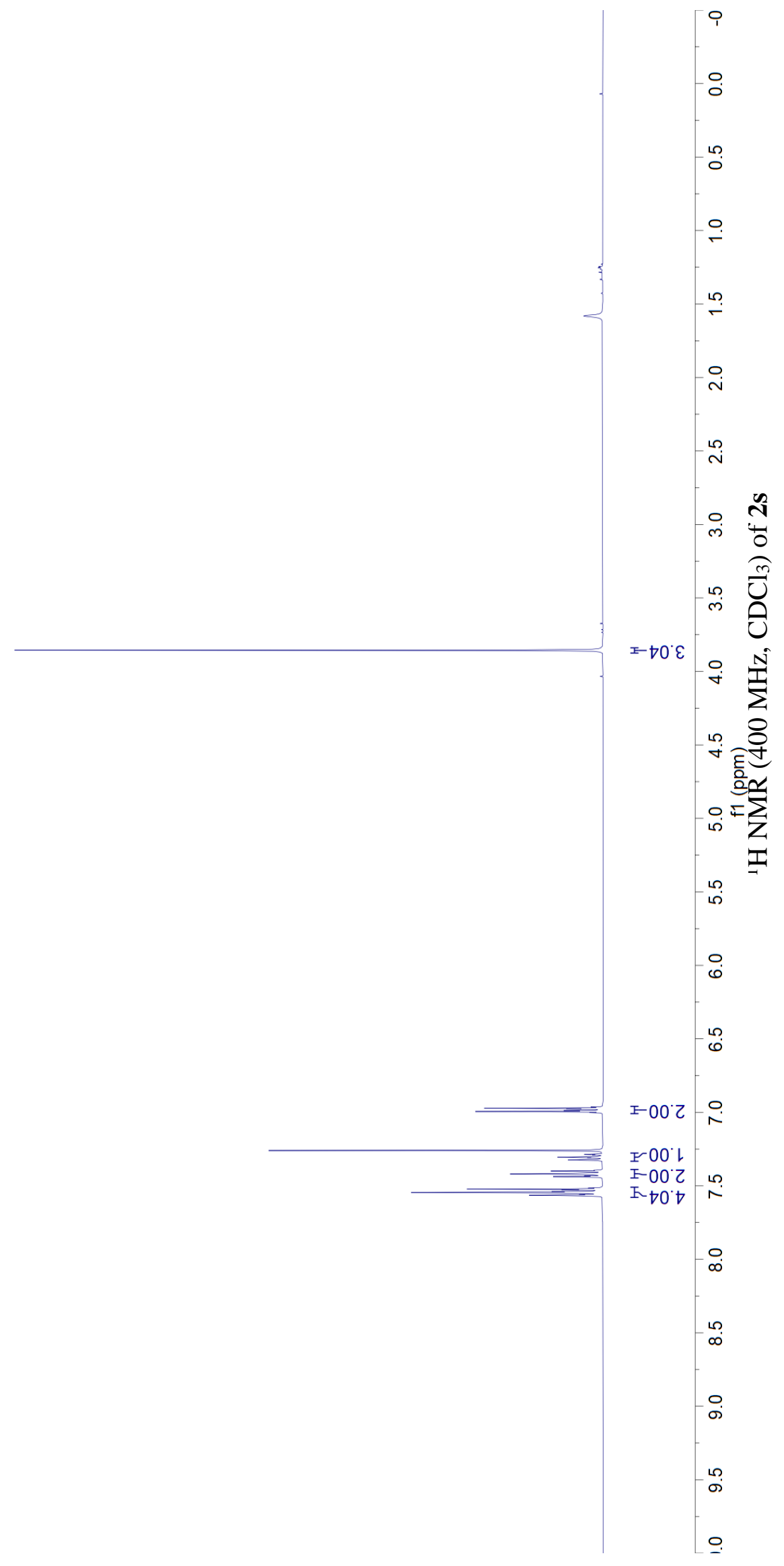




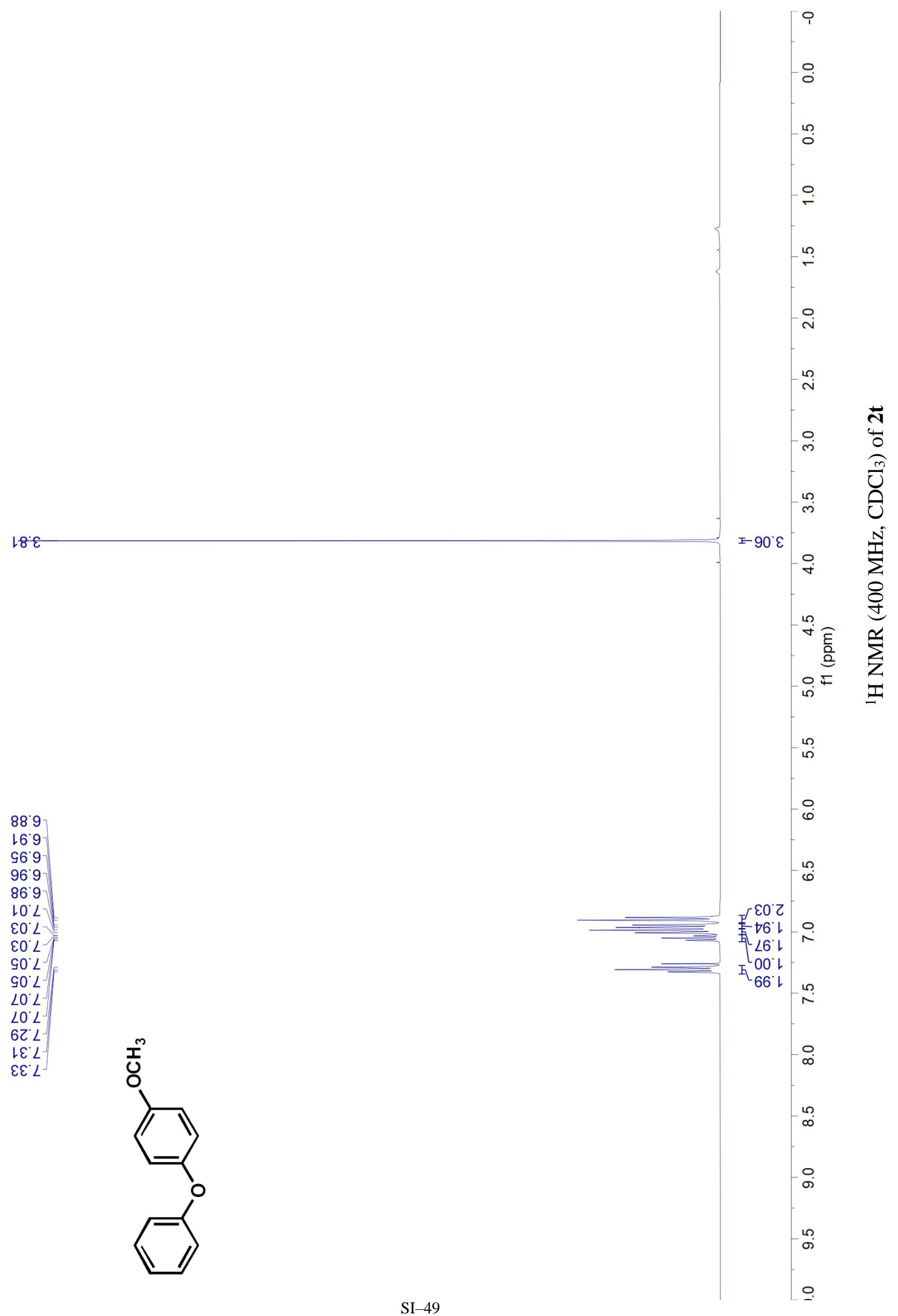




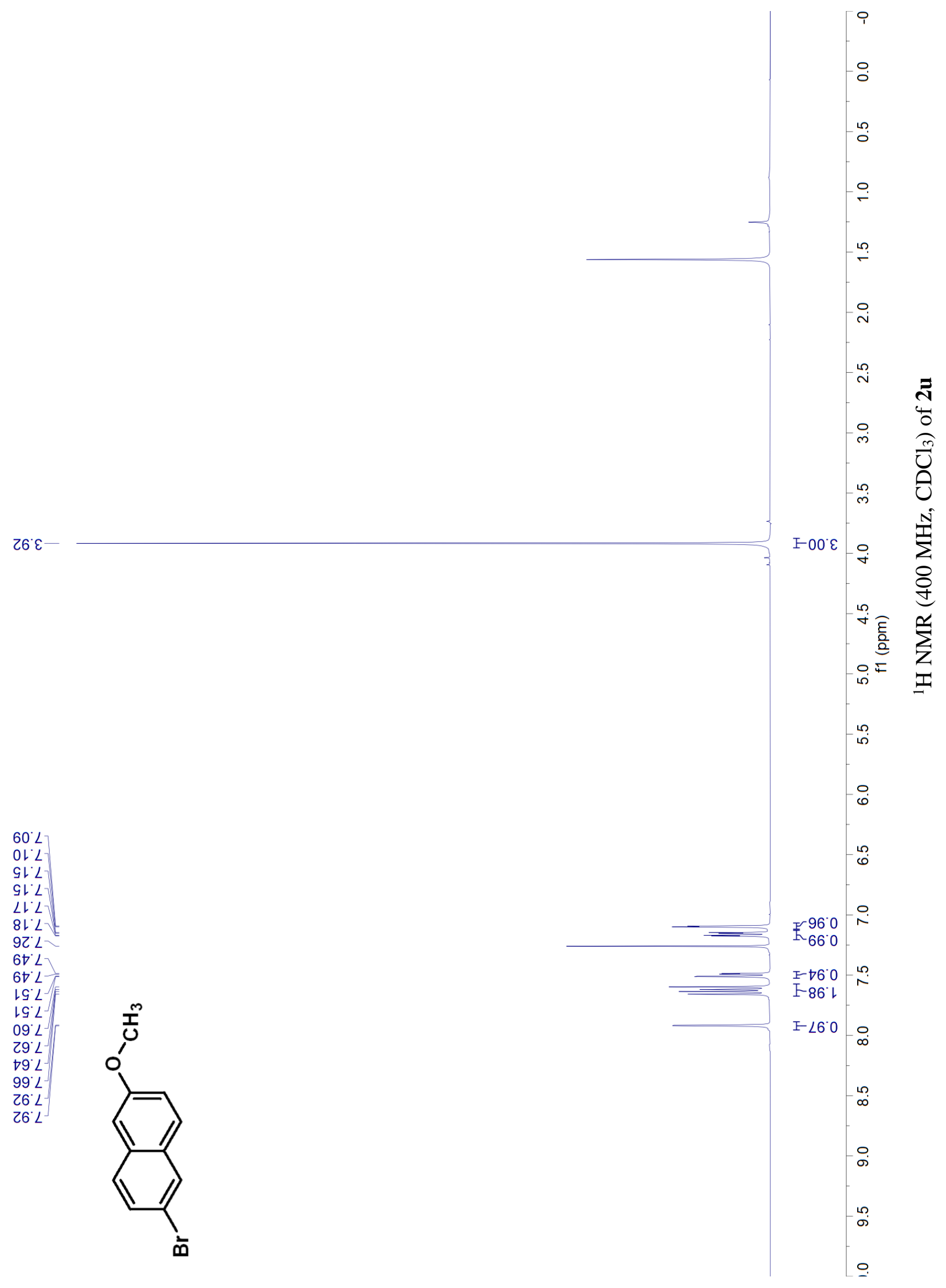




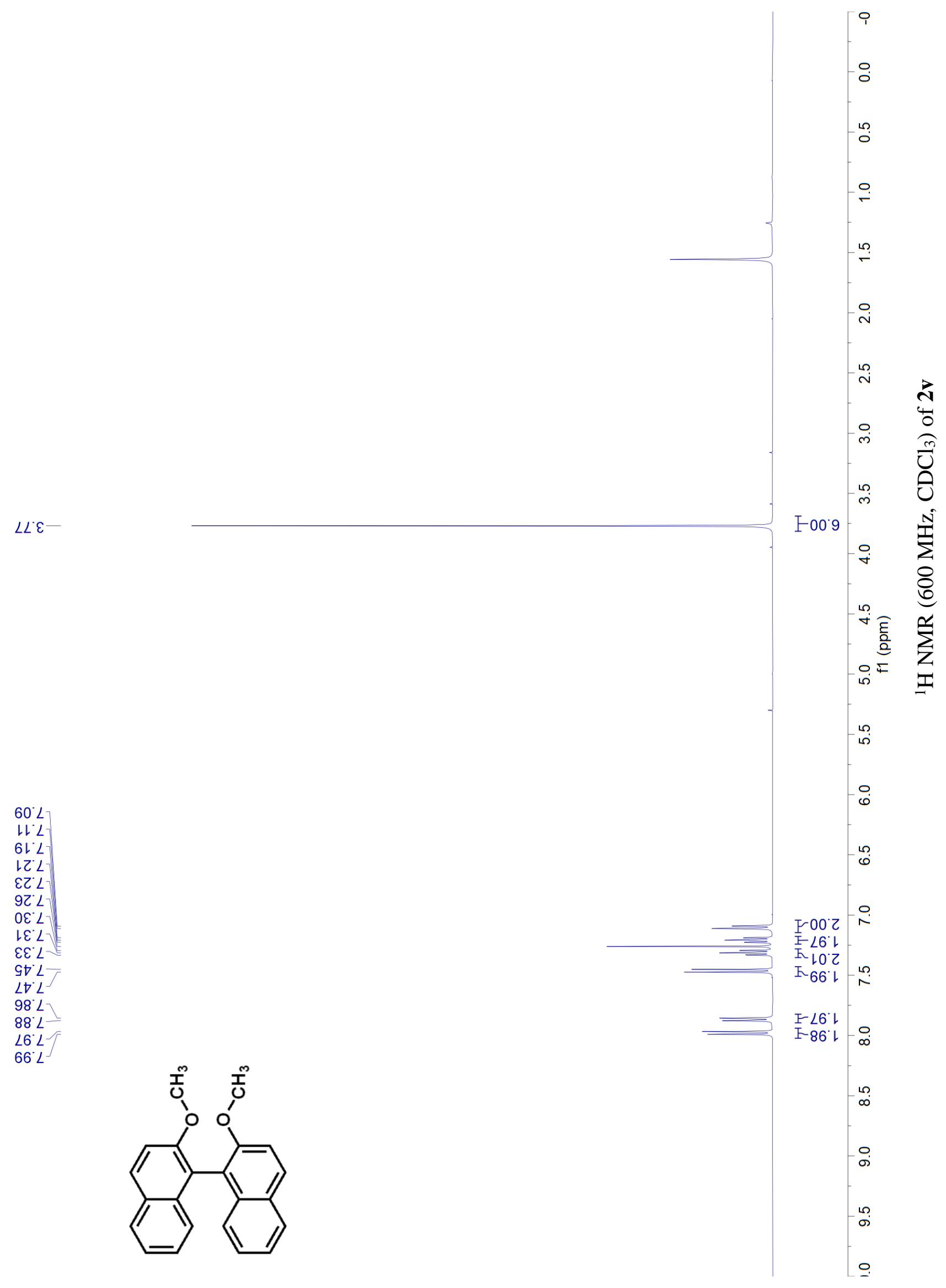




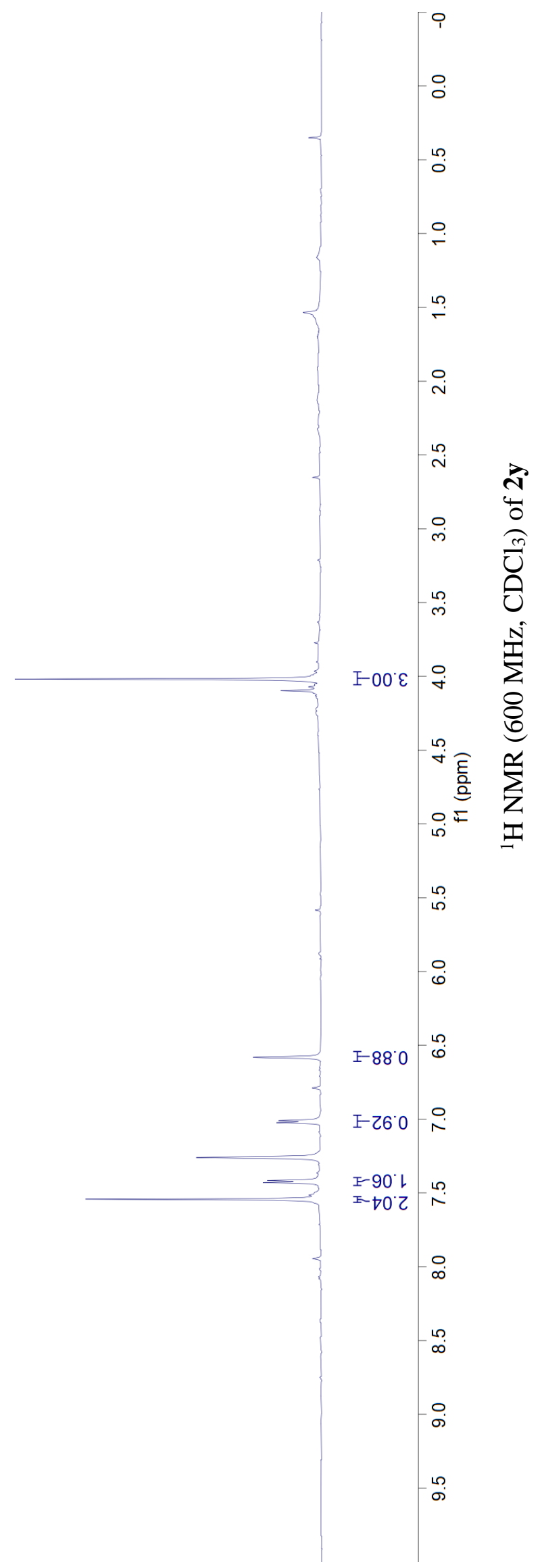




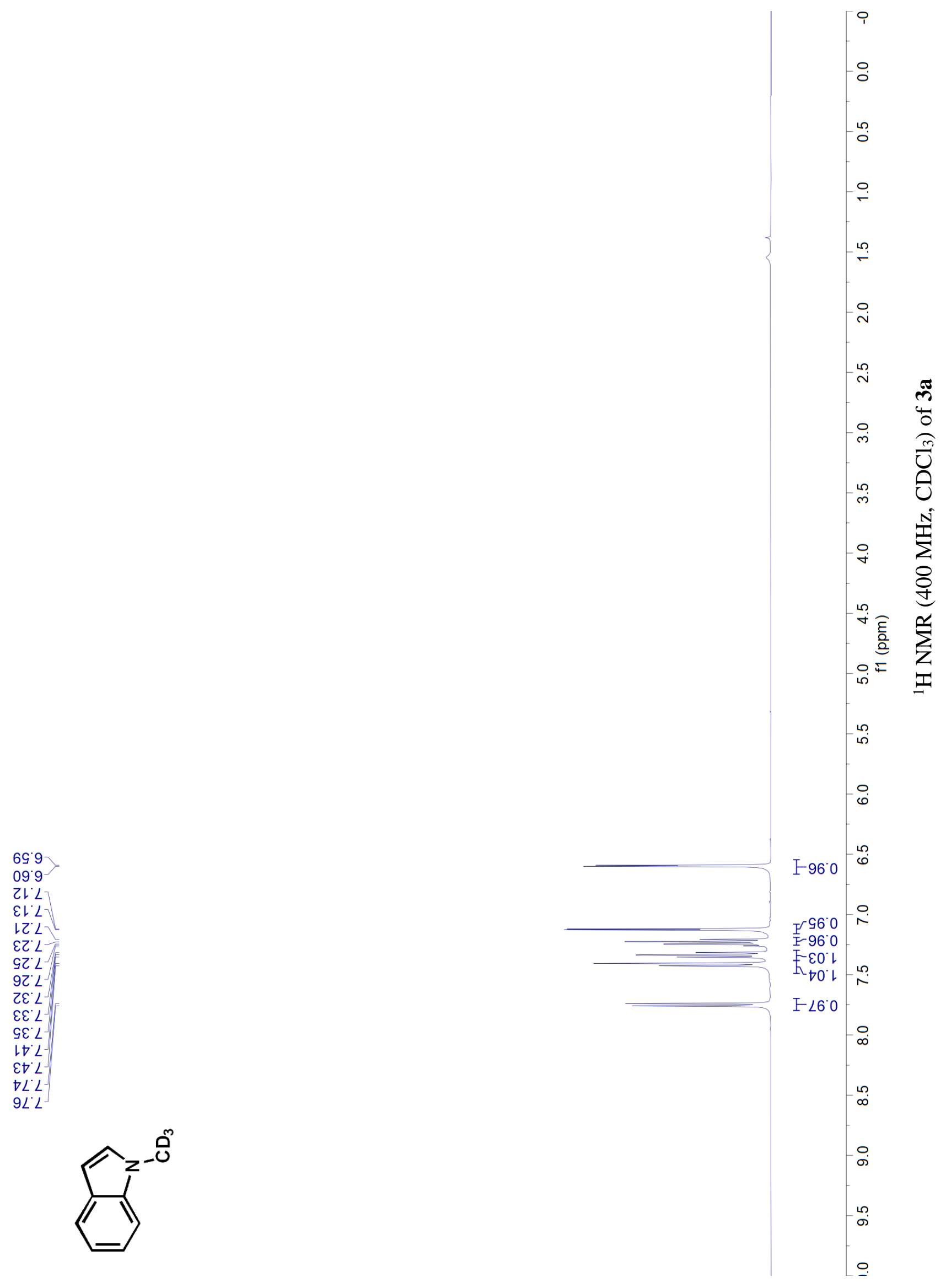


G6 $L$

9เ'ฉE

$\angle \varepsilon ' Z \varepsilon$

$8 \mathrm{~S}^{\prime} \textrm{ }$

$\nabla 8.9 L$

$9 L^{\circ} L L$

$8 \nabla L L$

$16001-$

$\angle Z 60 L-$

乙E. 6 L -

$\varepsilon 6.0 Z$ L

EG اZL

Z9'8ZL

†8 $8 \mathrm{ZL}$

$\varepsilon L \cdot 9 \varepsilon\llcorner-$

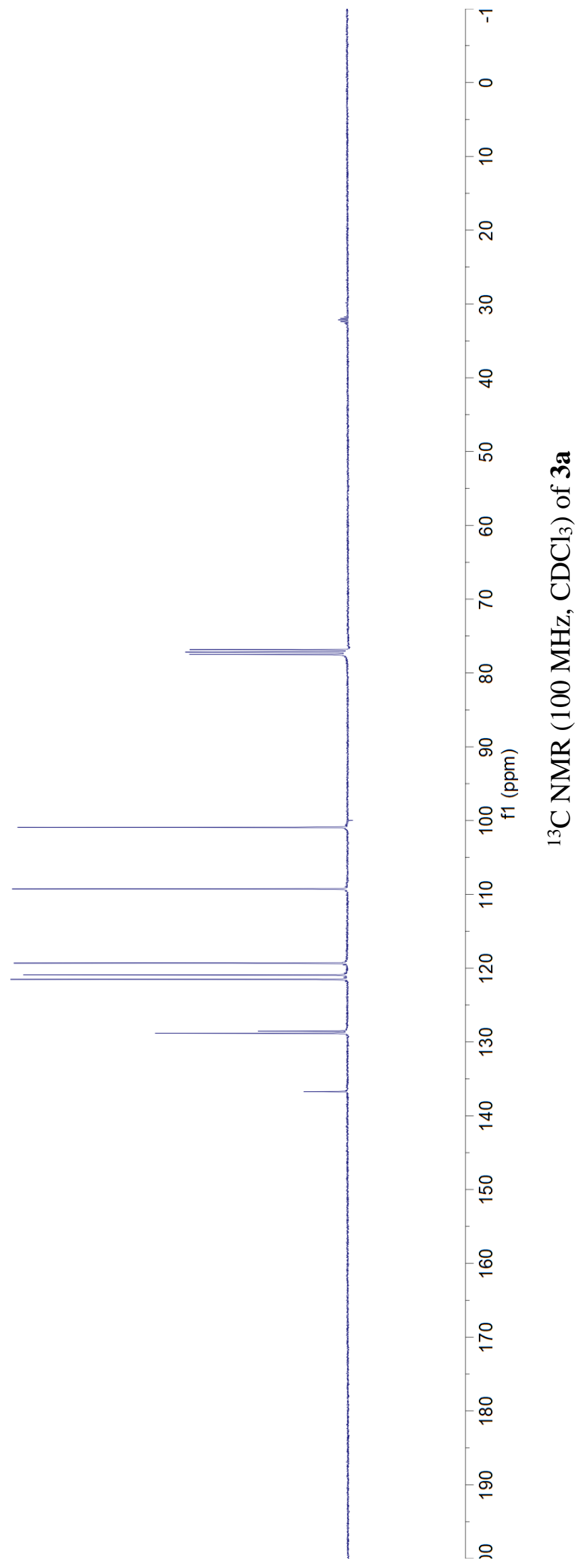



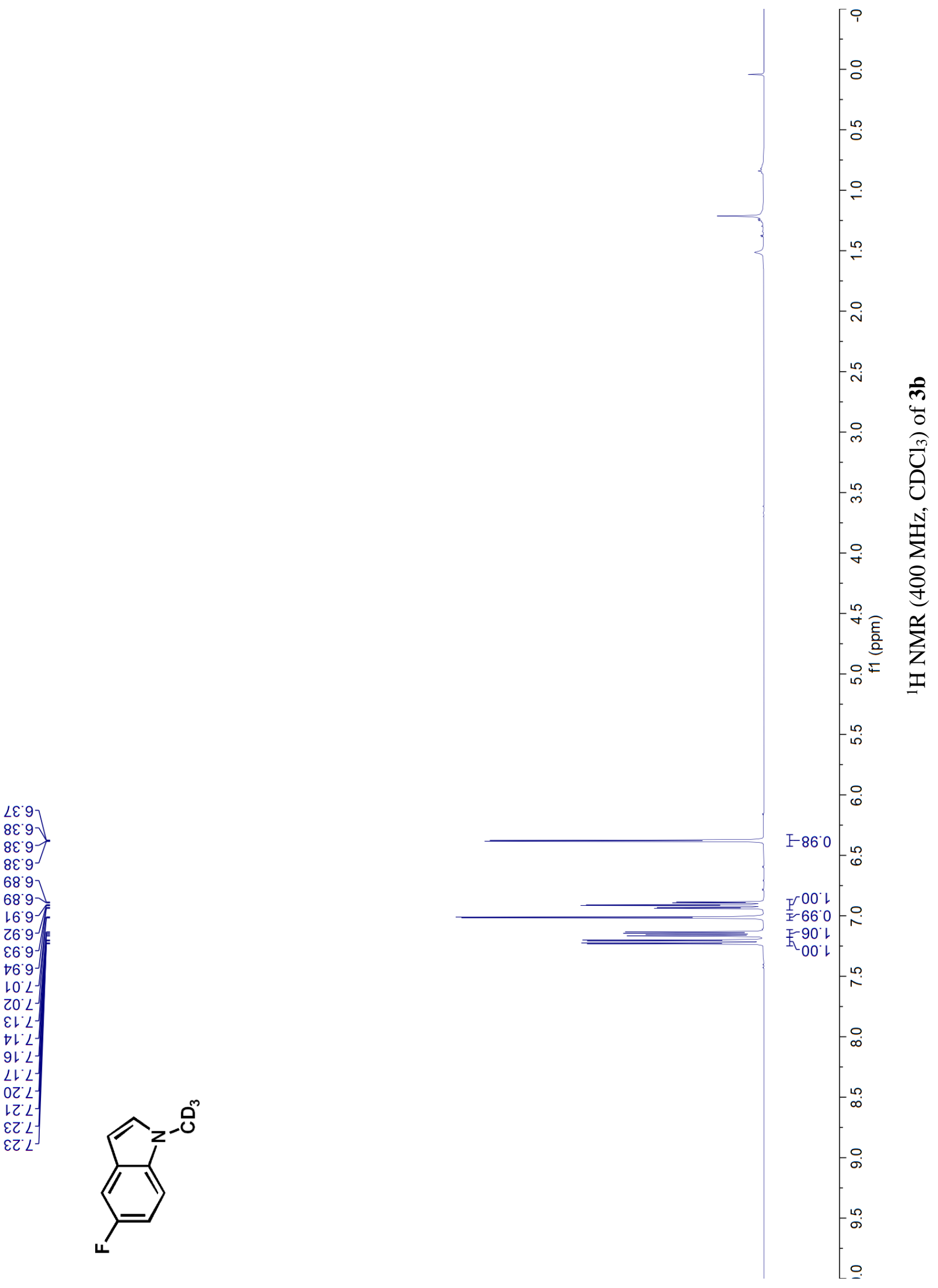
\&6 60 L

90 ㅇㄴ

$79^{\circ} 8$ ᄂ

$\checkmark L 8 Z$ L

97 0हL

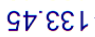

9L9GL-

8069 L

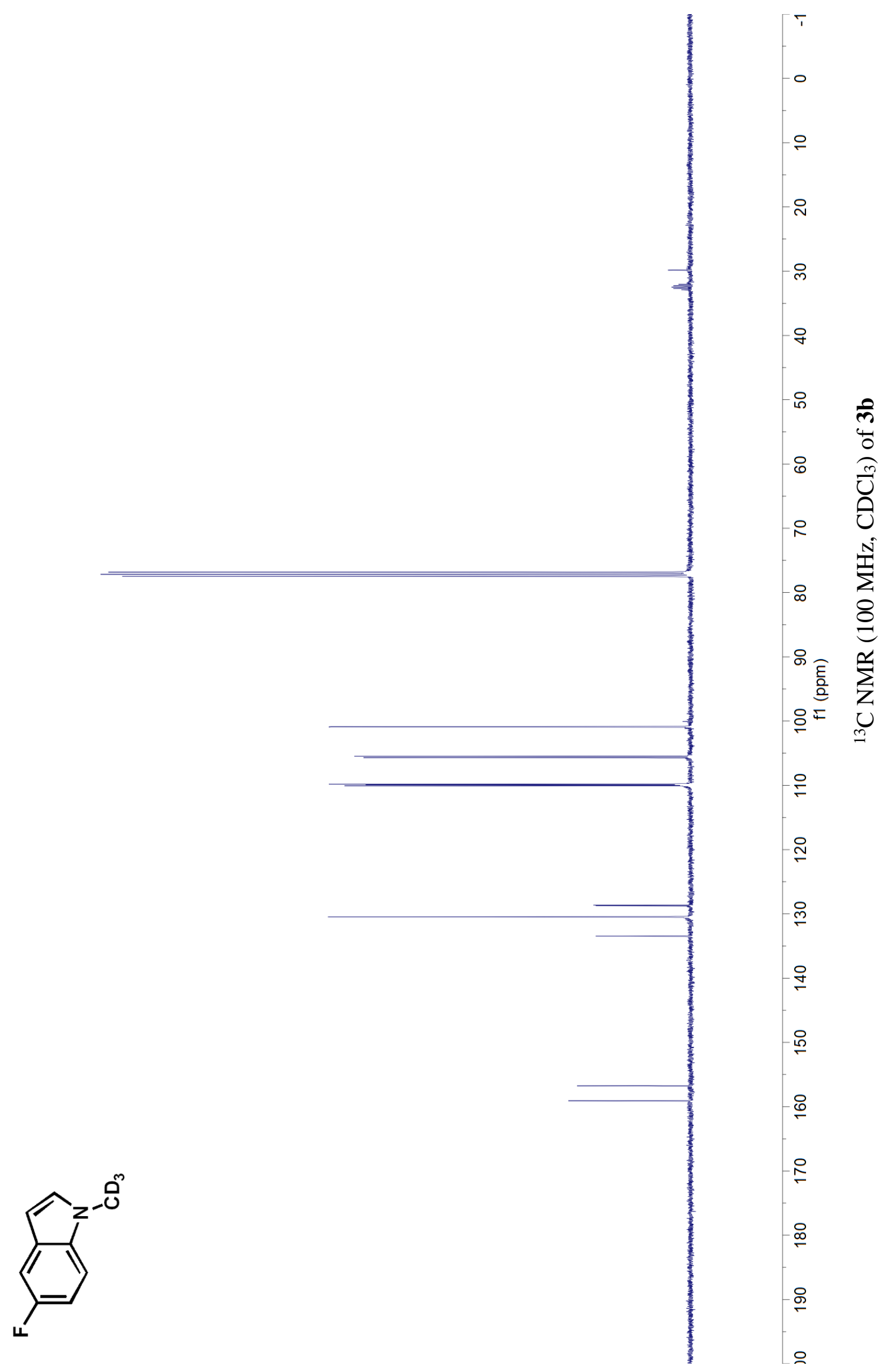




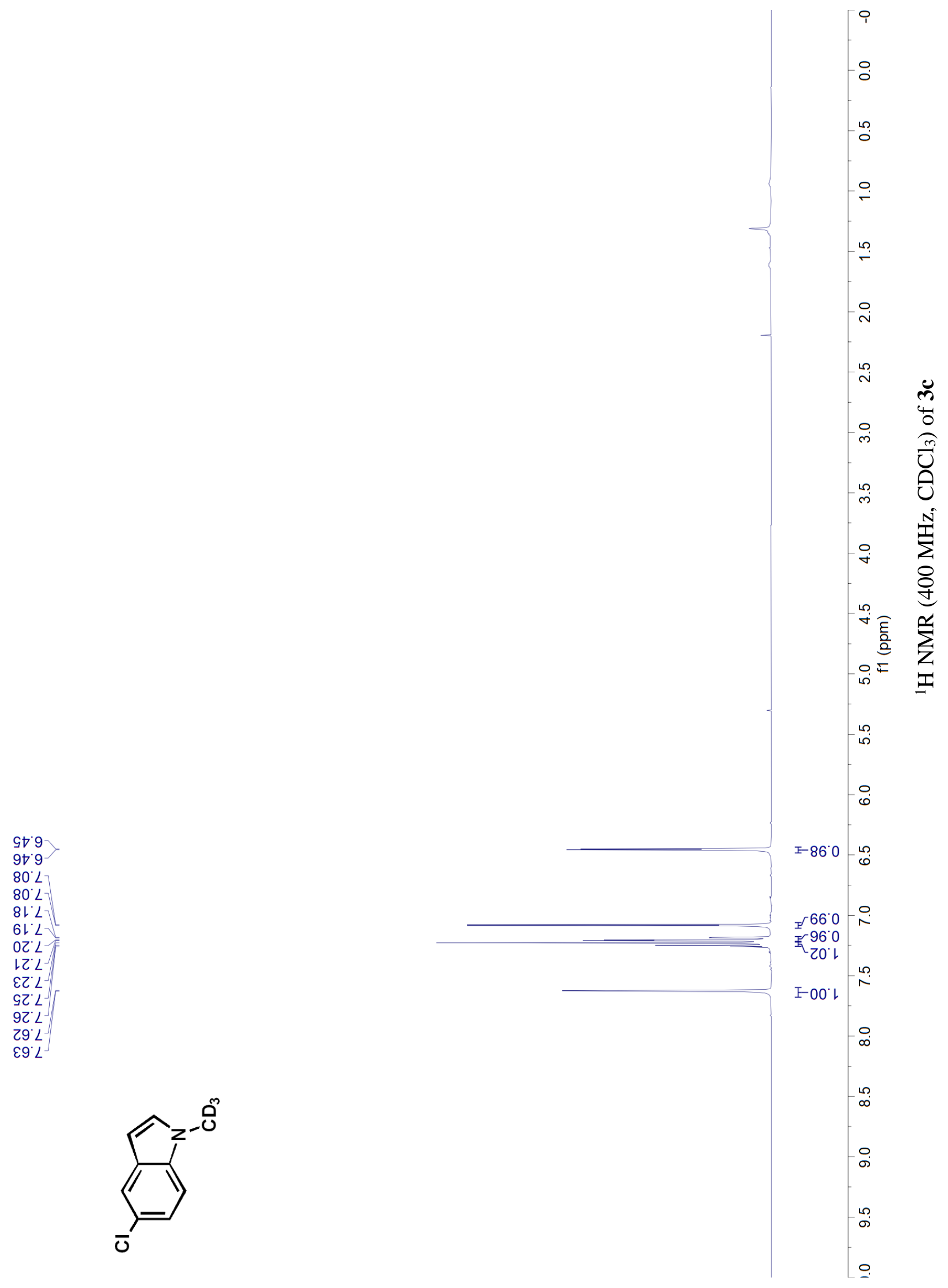




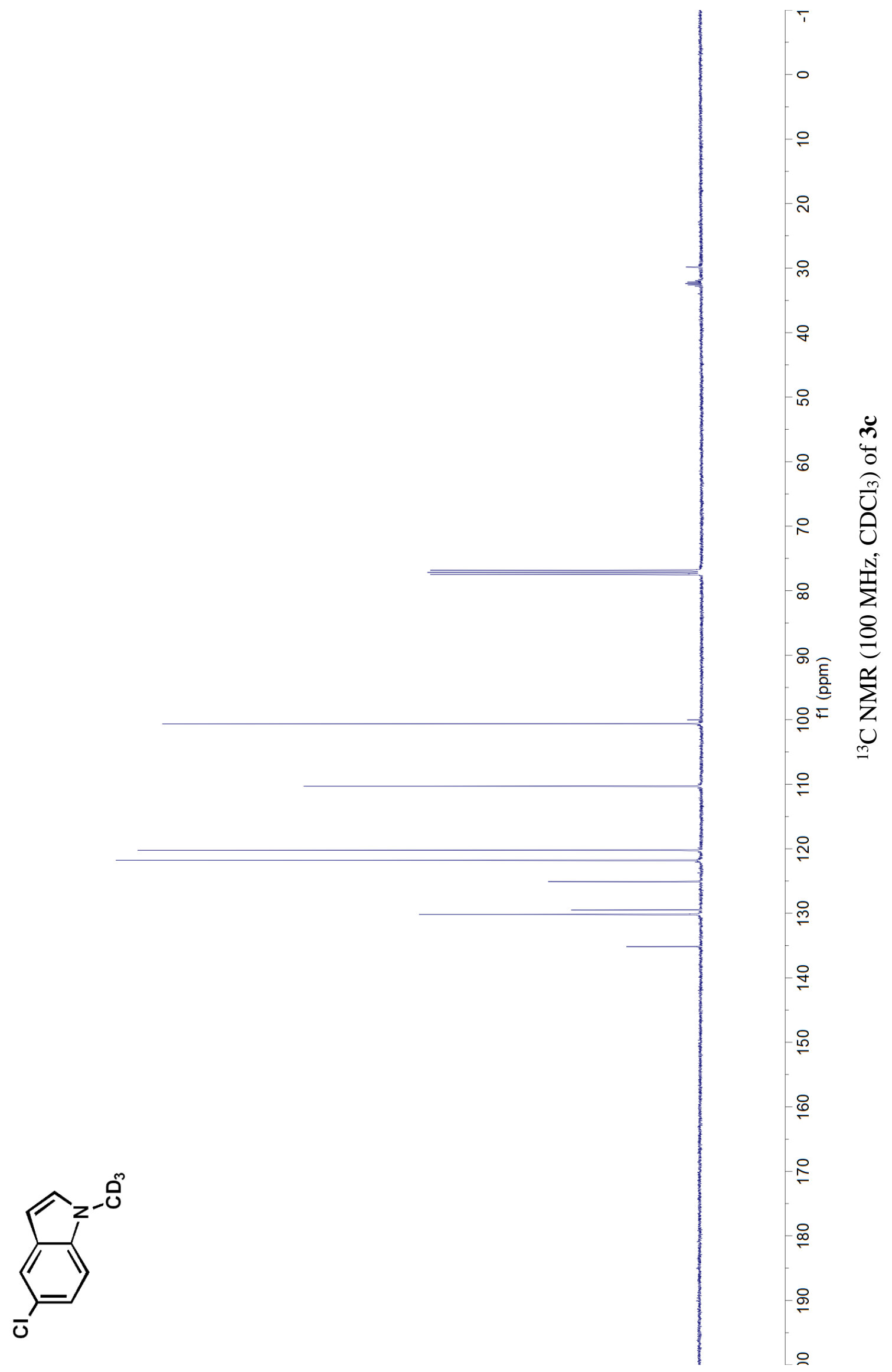



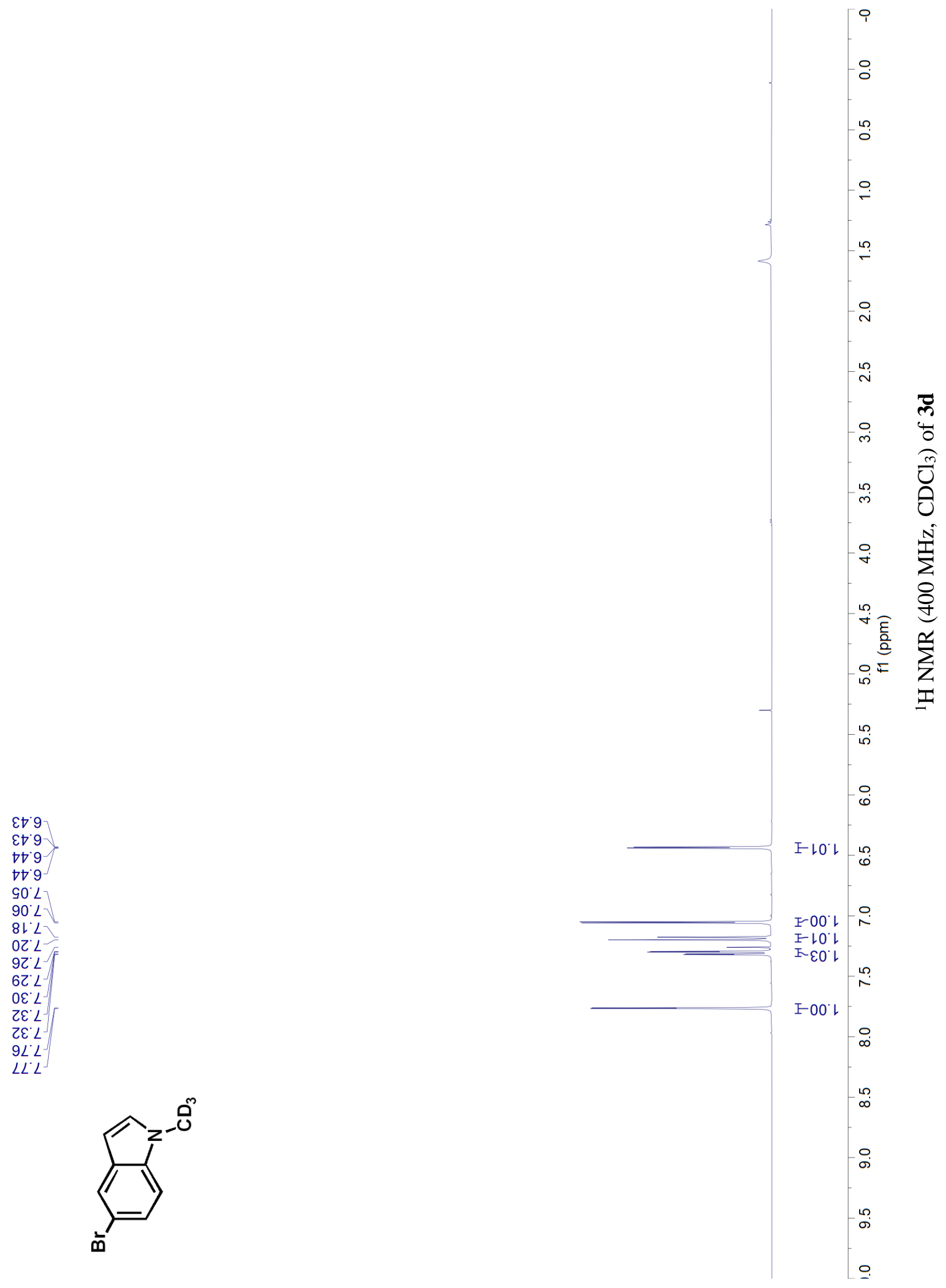
$96 \cdot \varepsilon$

$91.2 L$

$8 \nabla L L$

$99.001-$

LLOLL

OLZLL-

乙ย๕こレ-

$\varepsilon \varepsilon^{\circ} \triangleright Z \downarrow$

$\varepsilon 00^{\circ}$ -

9เ०८L-

Ot $9 \varepsilon$ L
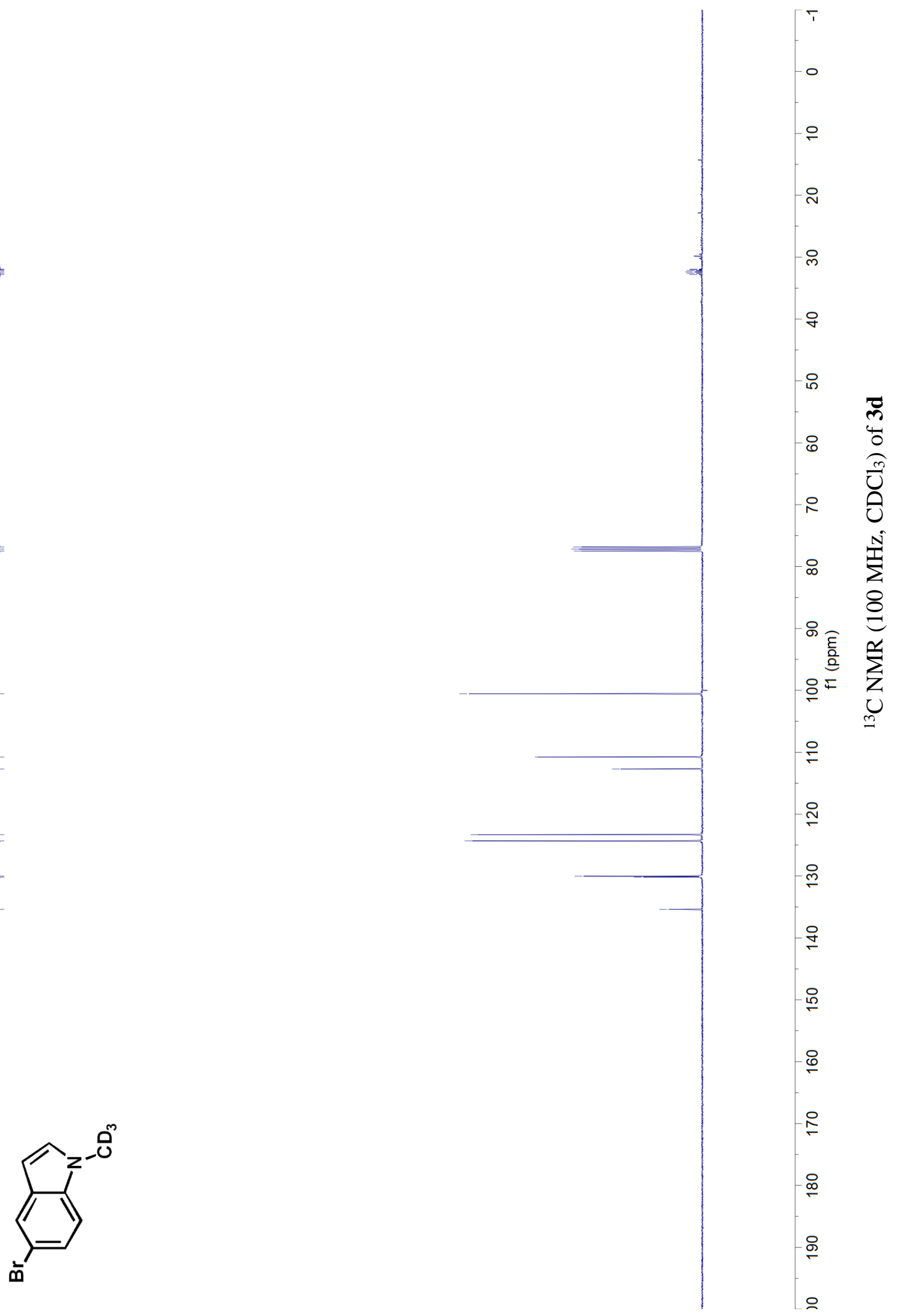

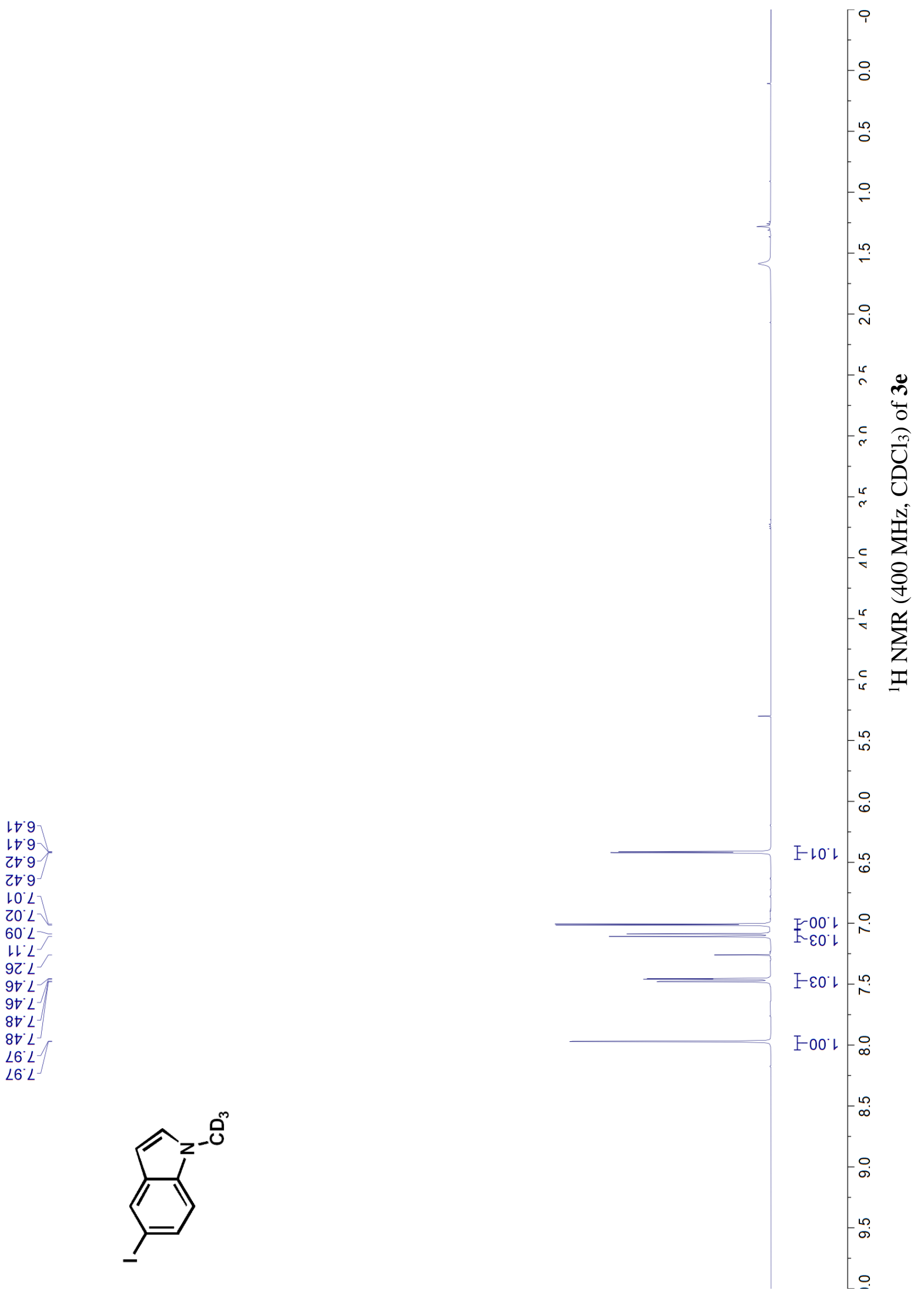
$87 L L$

$62001-$

ยะเレー

$\varepsilon 962$ L

9962L

GL'6ZL-

$\nabla 0^{\circ} L E L$

$6 L \mathrm{GL}$

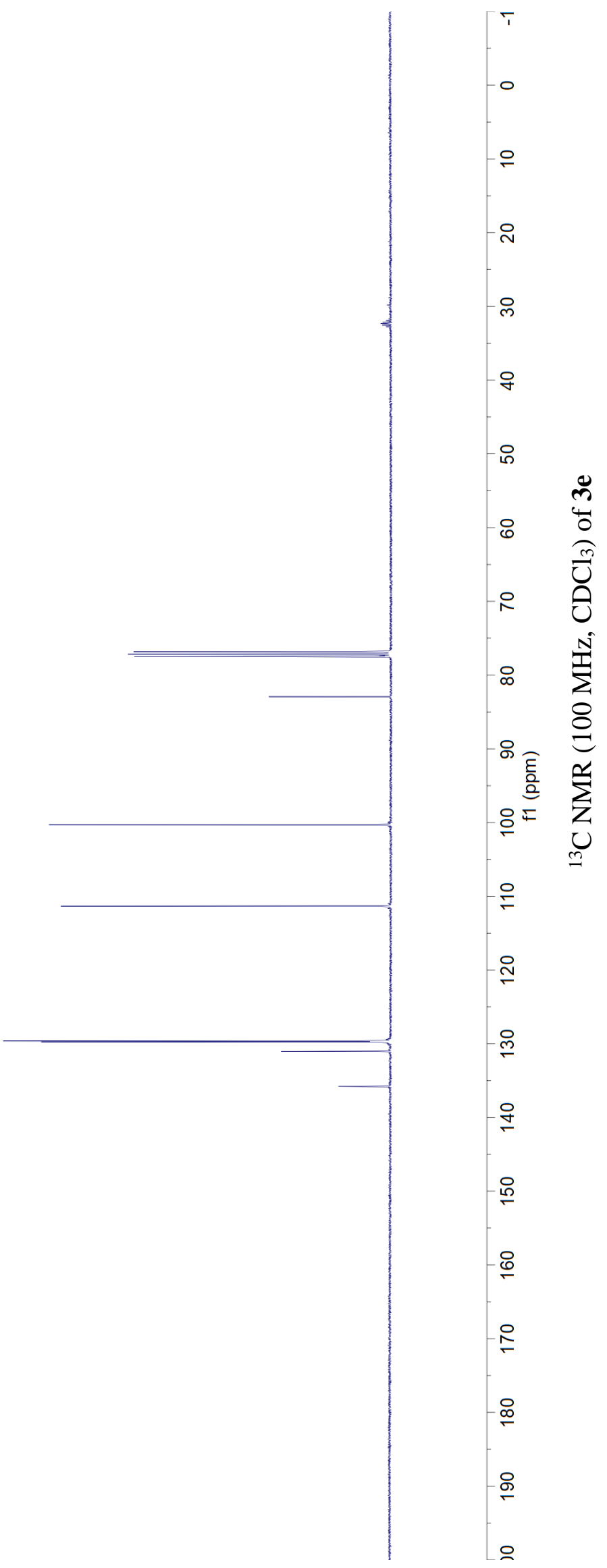




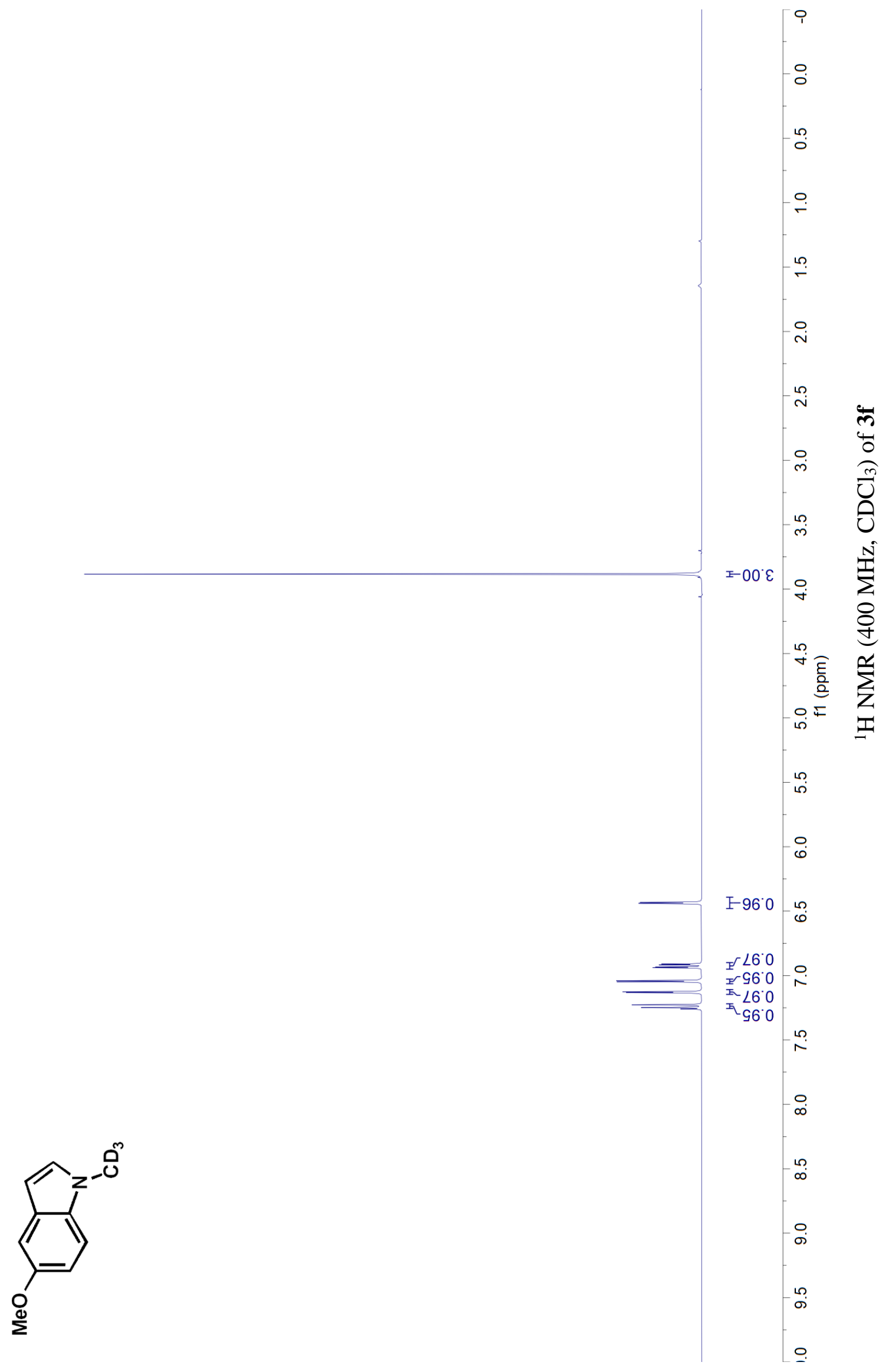




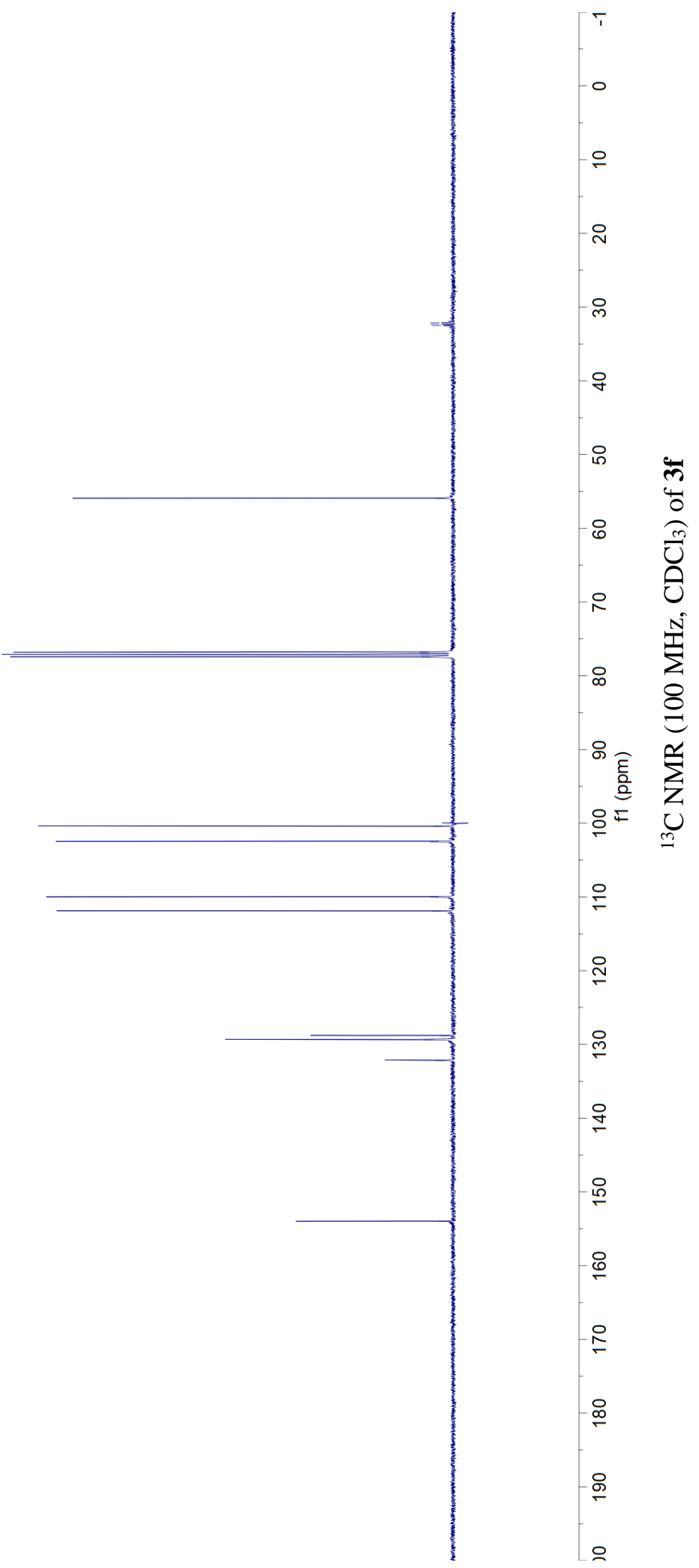



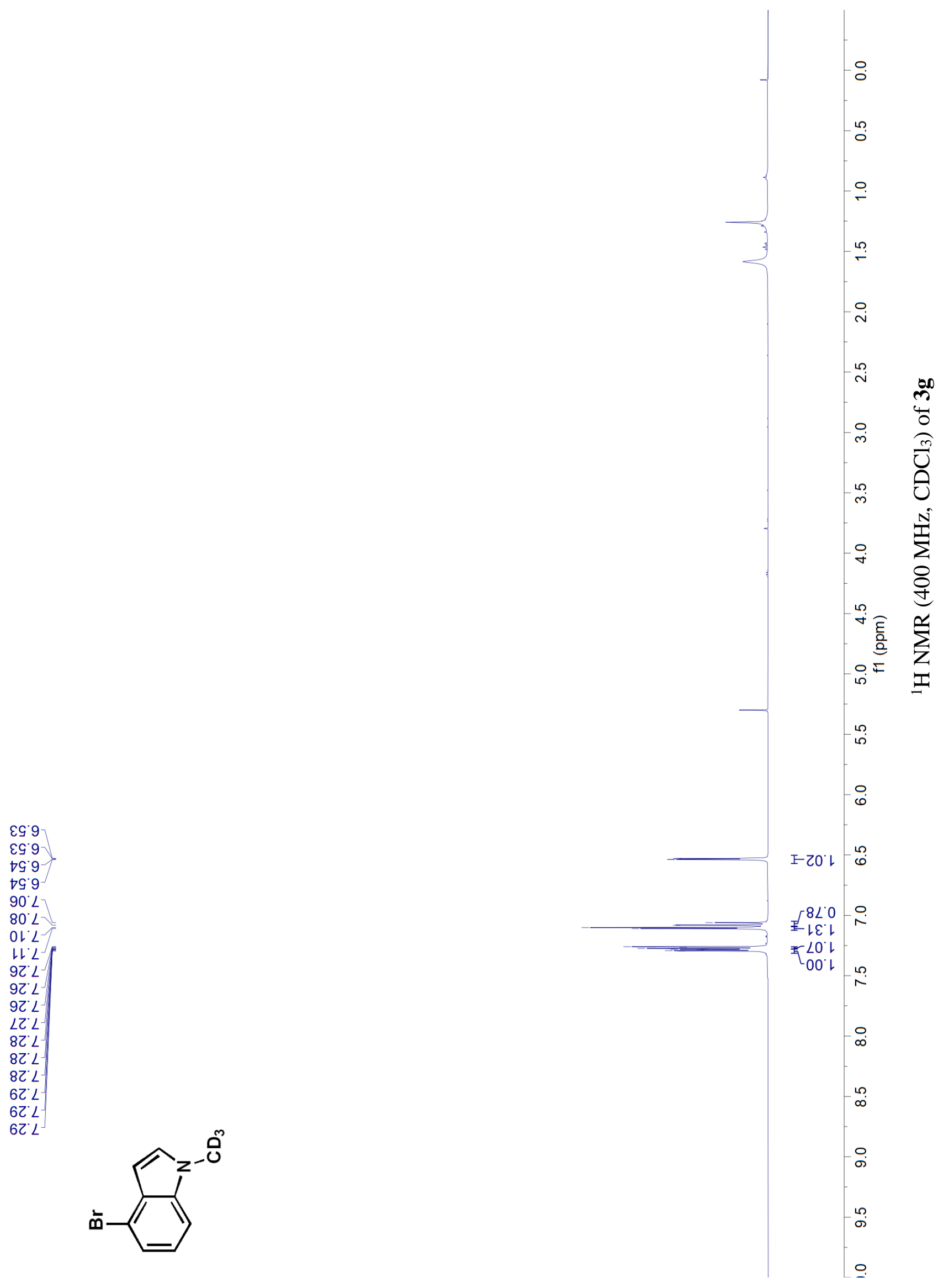
ยง ฉع-

$\nabla 8.9 L$

9LLL-

$87 L L$

ItレOL

${ }^{\circ} \mathrm{G}^{\circ} 80 \mathrm{~L}-$

ย6・เレー

0๕'ZZレ

st ZZL

0Z 6ZL

Et 6ZL

$60^{\circ} L E L-$

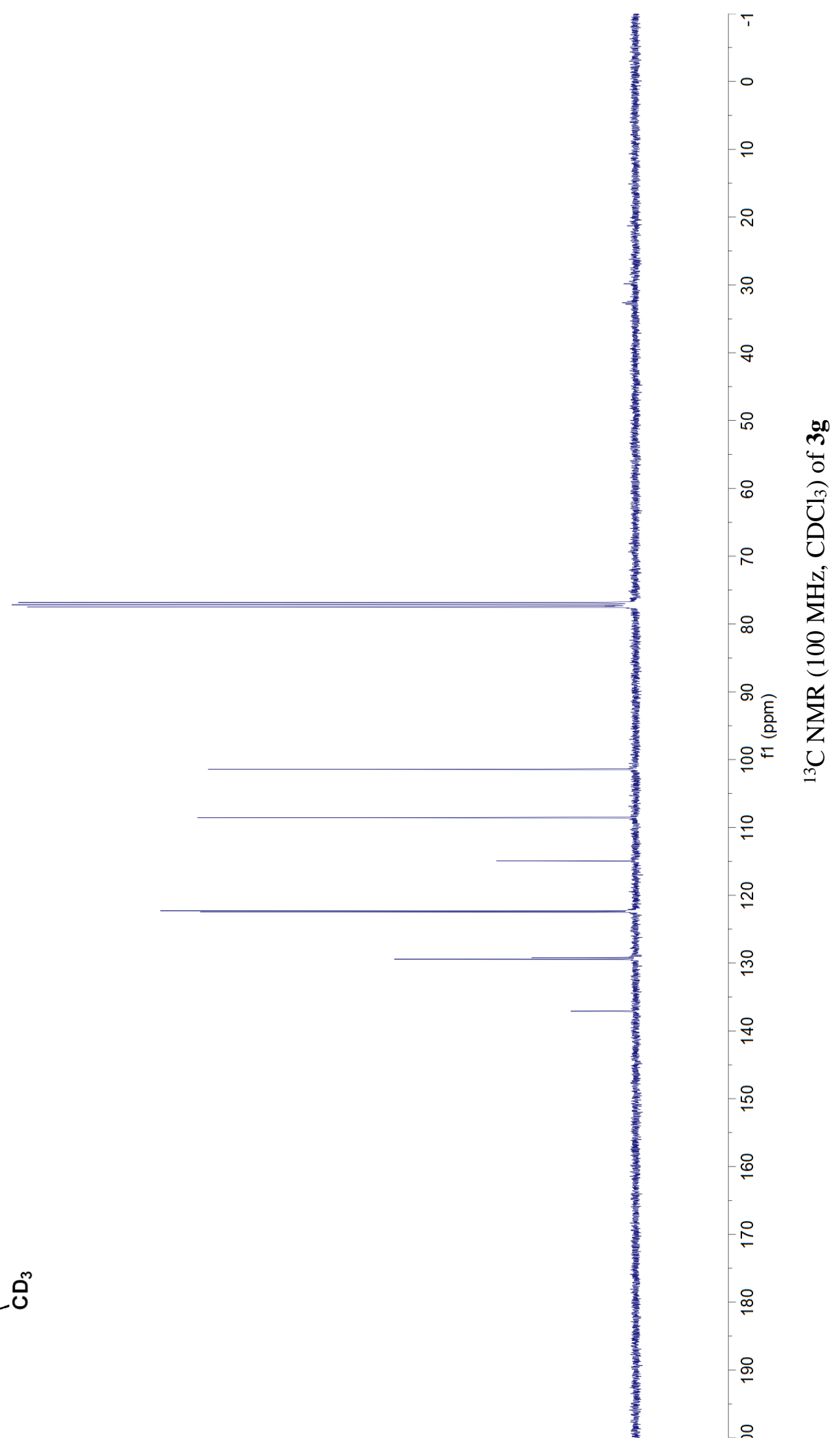




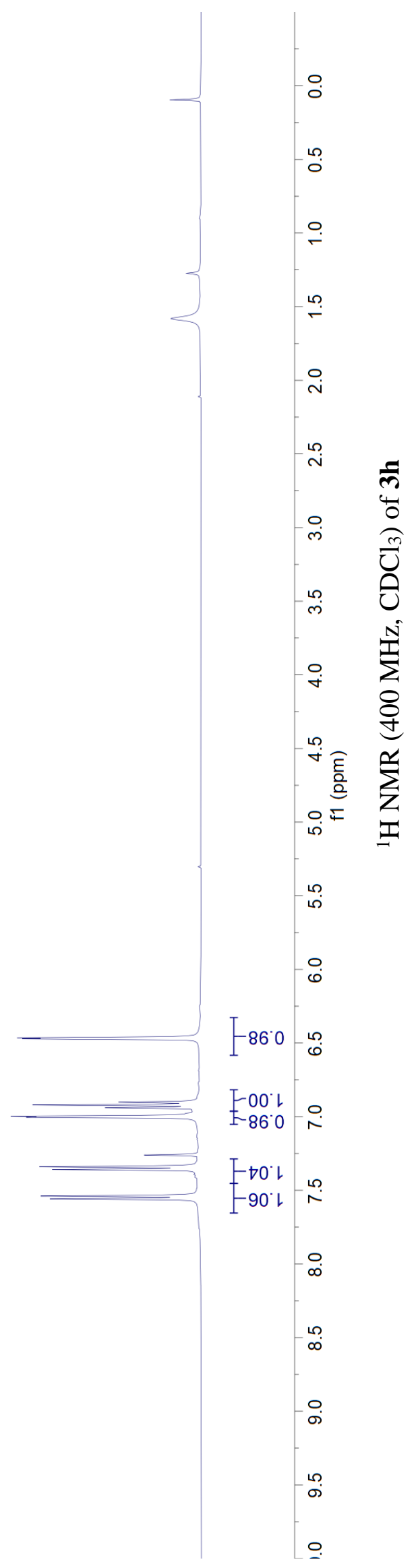




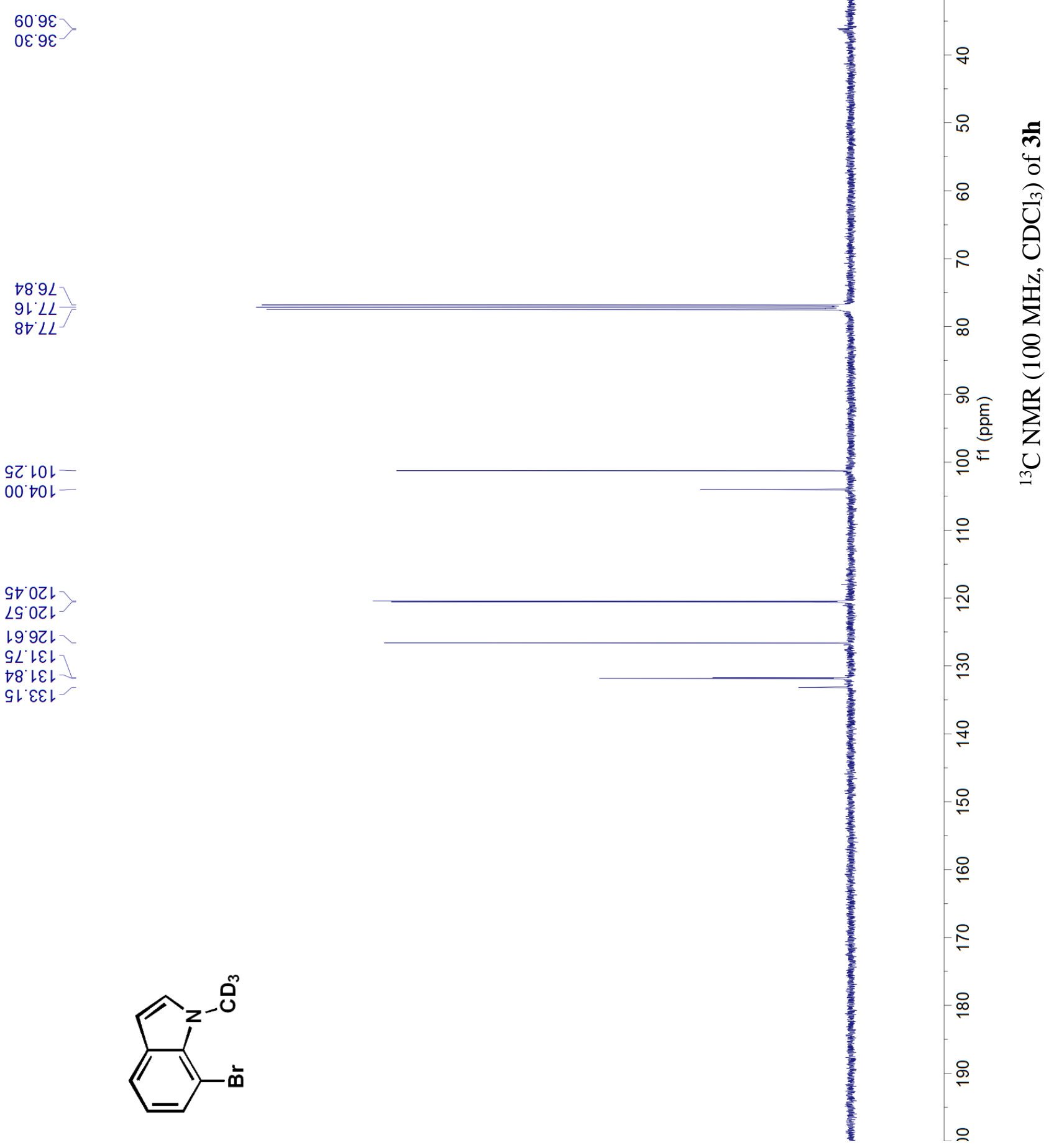




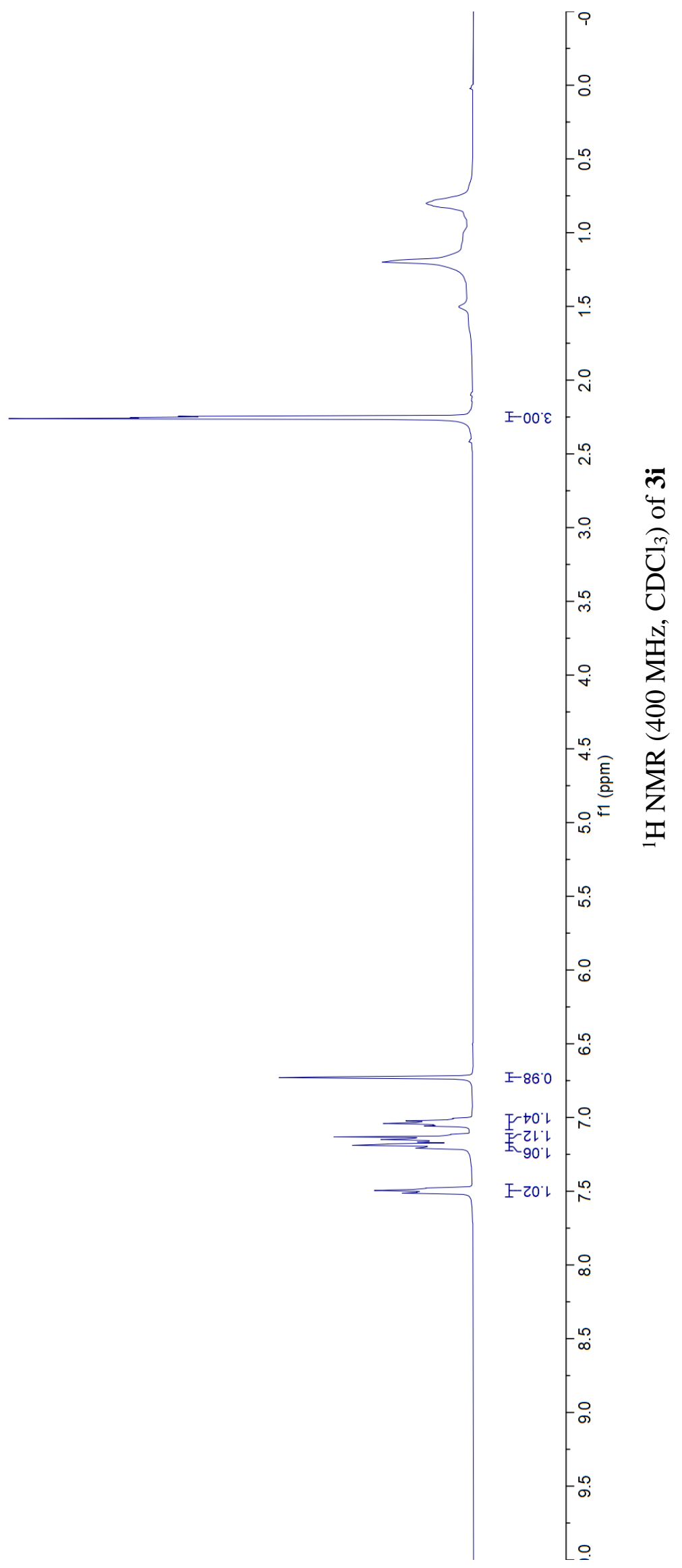


$896-$

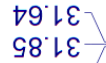

$90 \cdot 2 \varepsilon$

98. $9 L$

$\angle L L L$

$8 \nabla L L$

$6060 \mathrm{~L}$

El.0lL

$\angle S^{\circ} 8 \mathrm{LL}$

206 L

8大 IZL

$\angle G$ GZL

LL'8ZL

SO $L E L-$

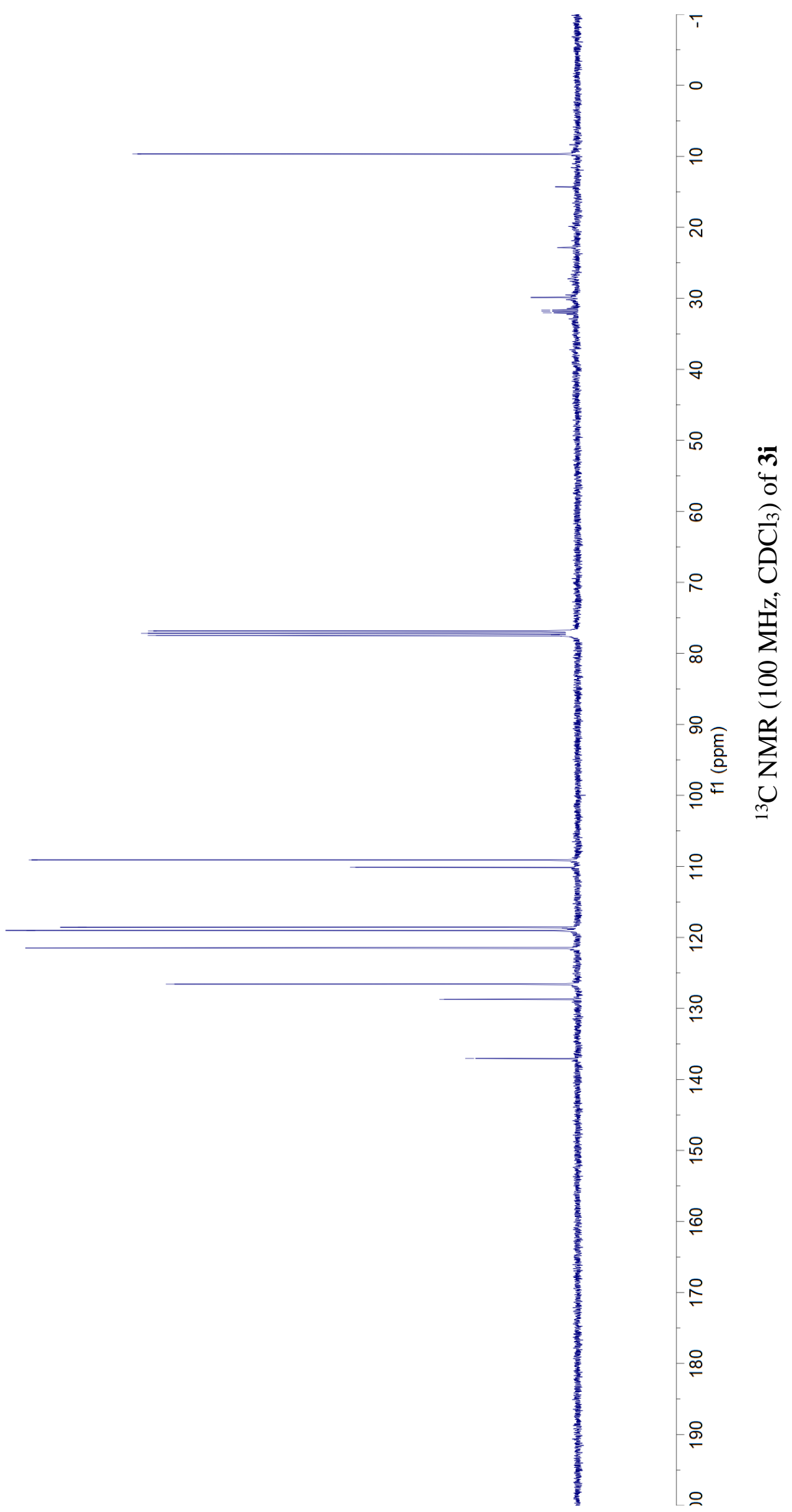

SI-70 

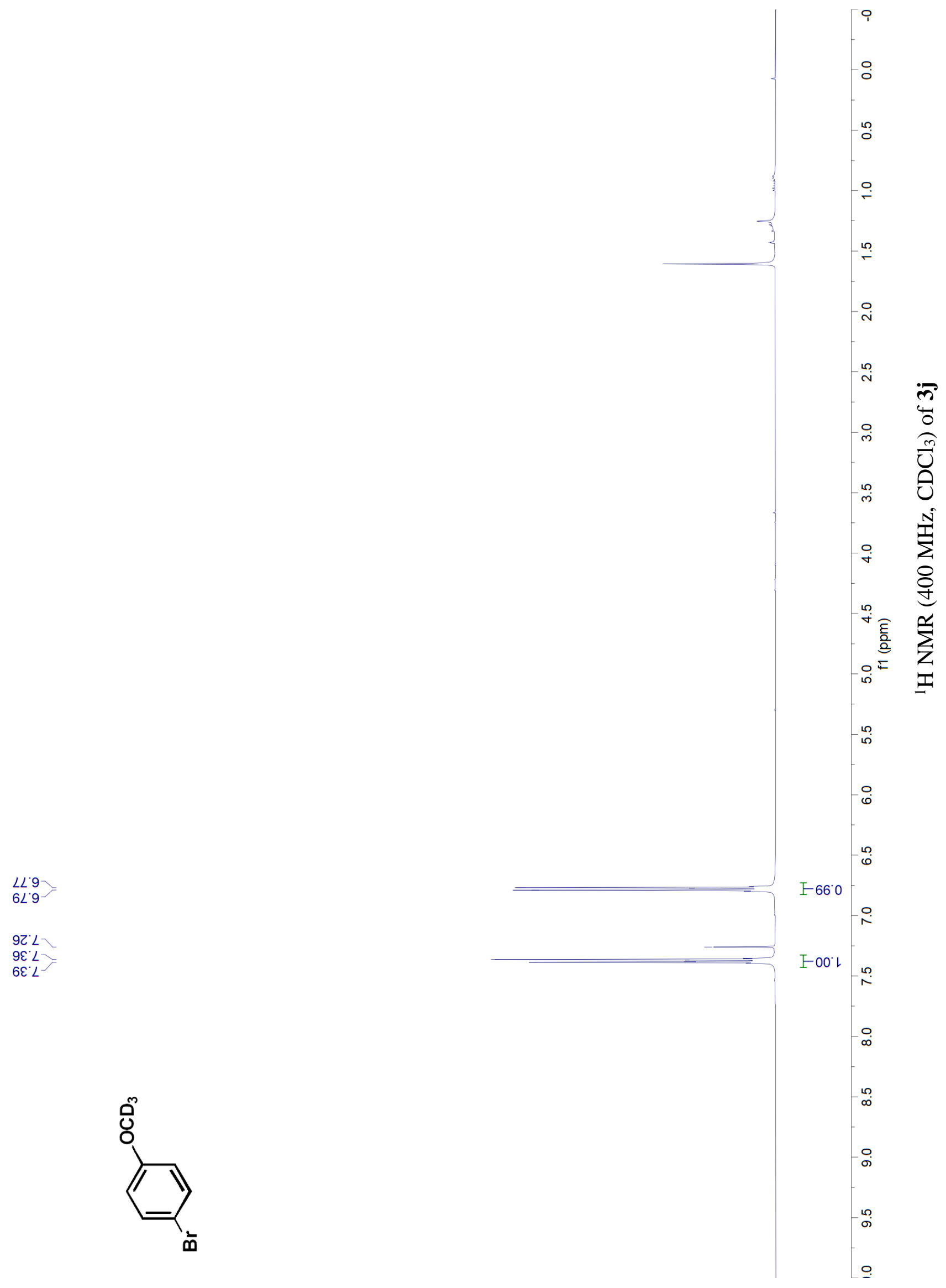


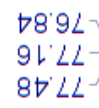

$9 L^{\prime} L L$

$8 \nabla L L$

68てレ-

เ8'งL-

ธะ'วยเ-

9L'89L-

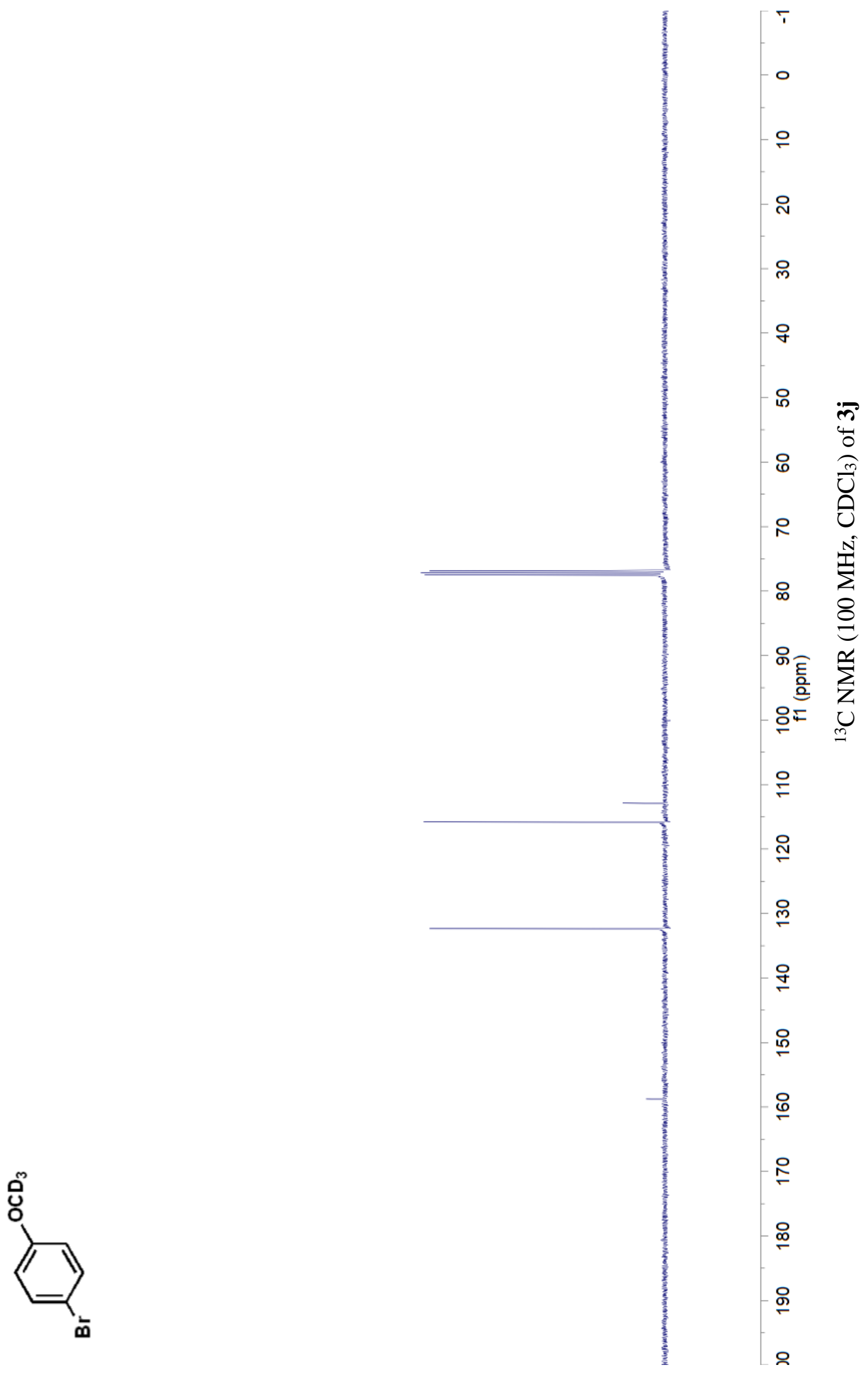



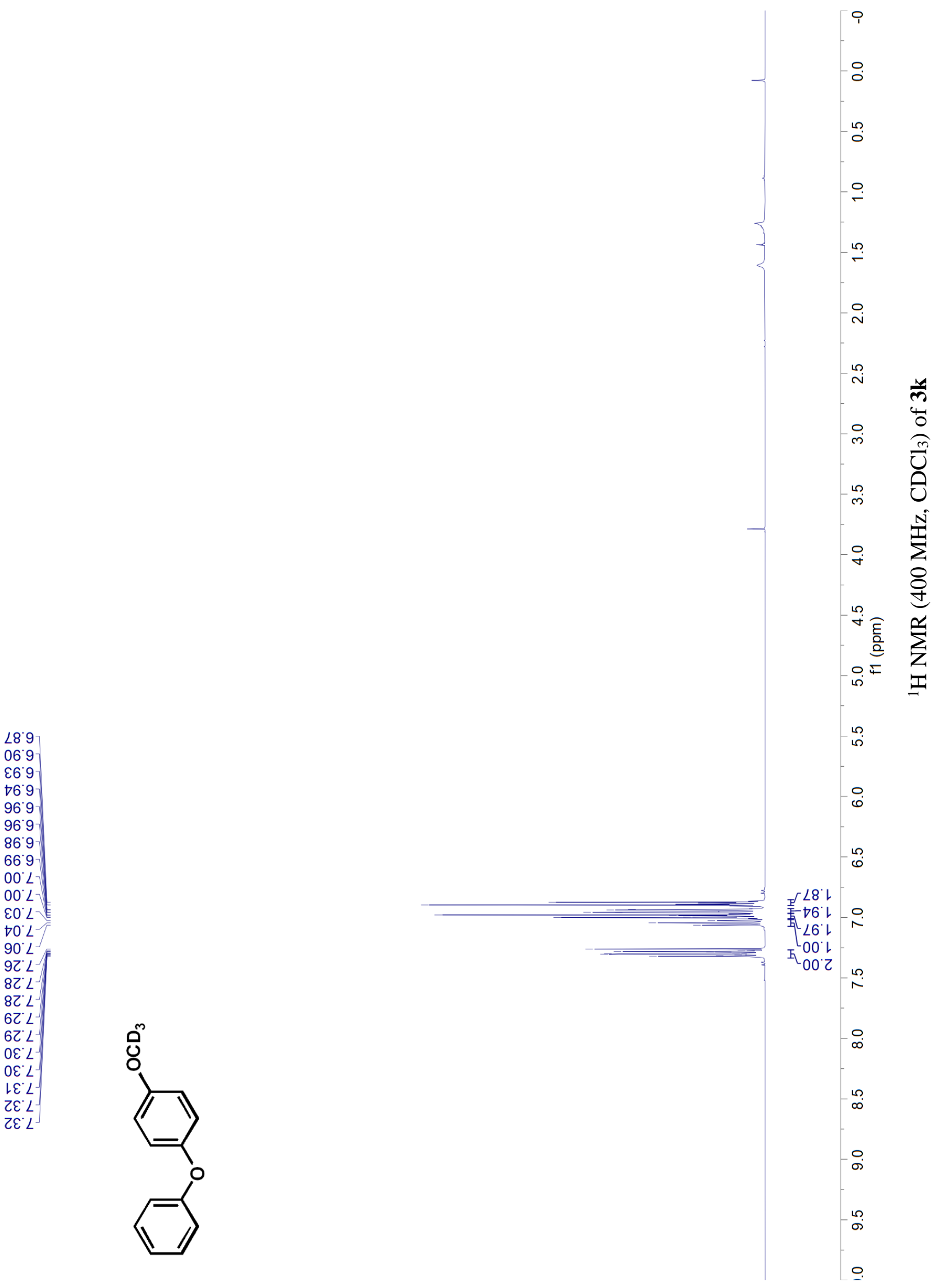
$\nabla 89 L$

$912 L$
$87 L L$

จ6ナレー

$\angle 9 \angle L$

$00^{\circ}$ 乙L

$\nabla S Z Z 1-$

$\nabla L 62 レ-$

LLOSL-

00 '99L-

s9.89. -

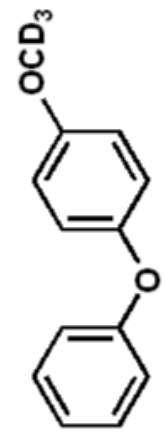

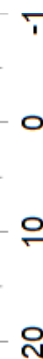

이

우

요

(

8

웅

○

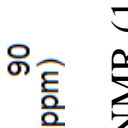

은 U

읃

ํำ

옴

움

음

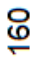

$\stackrel{ }{ }$

$\stackrel{\circ}{\stackrel{0}{-1}}$

옴

ㅇ 


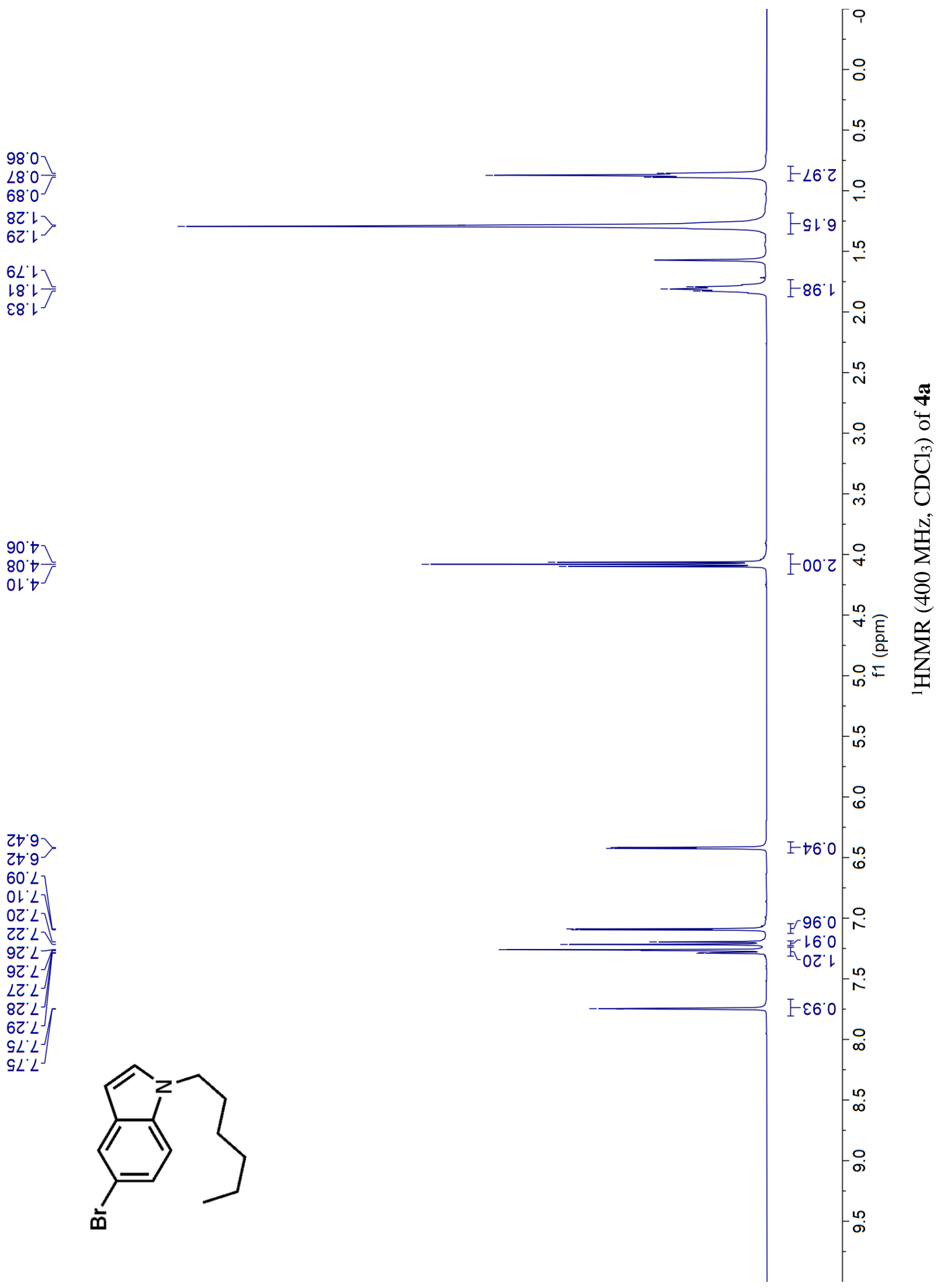



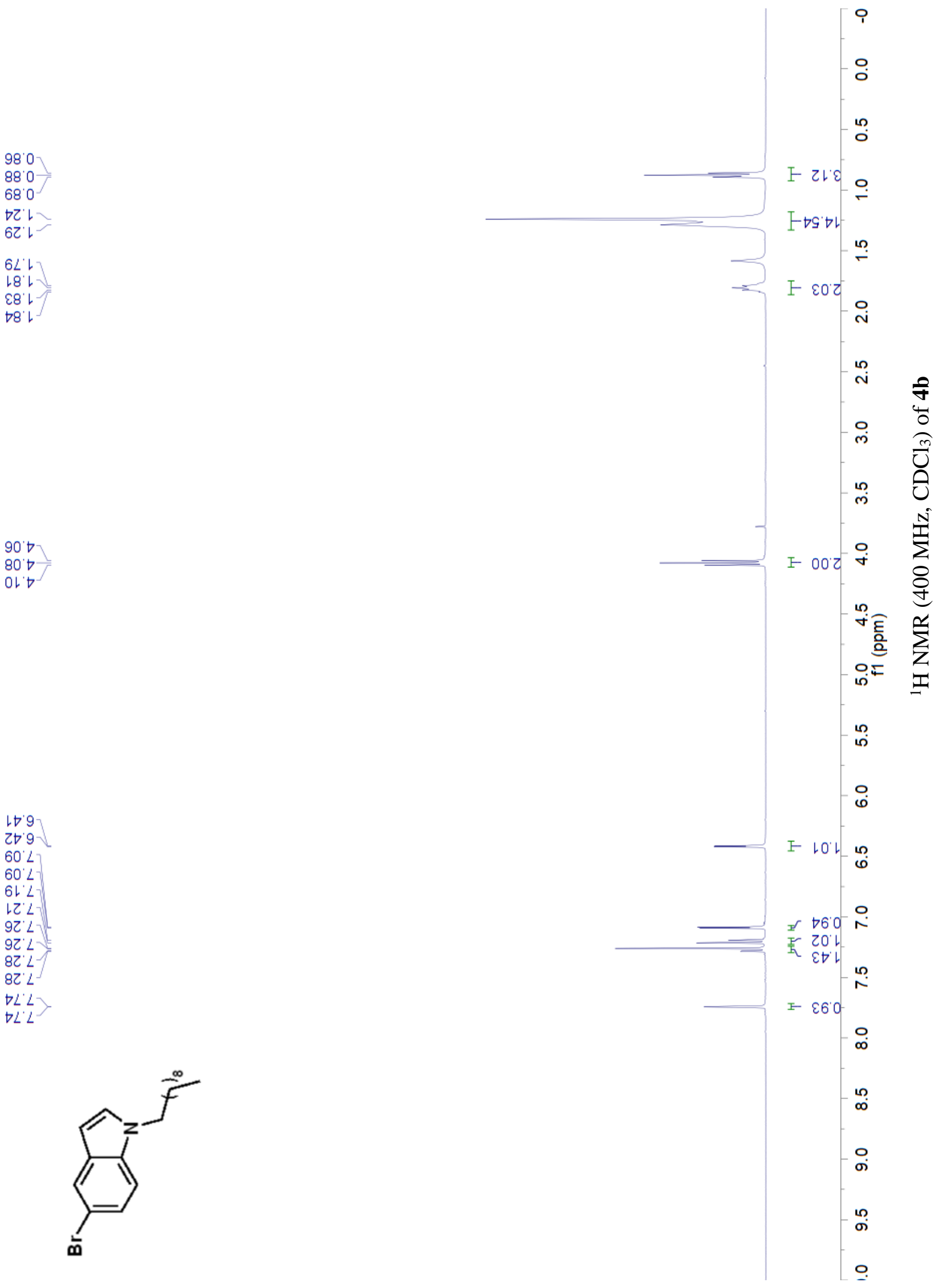

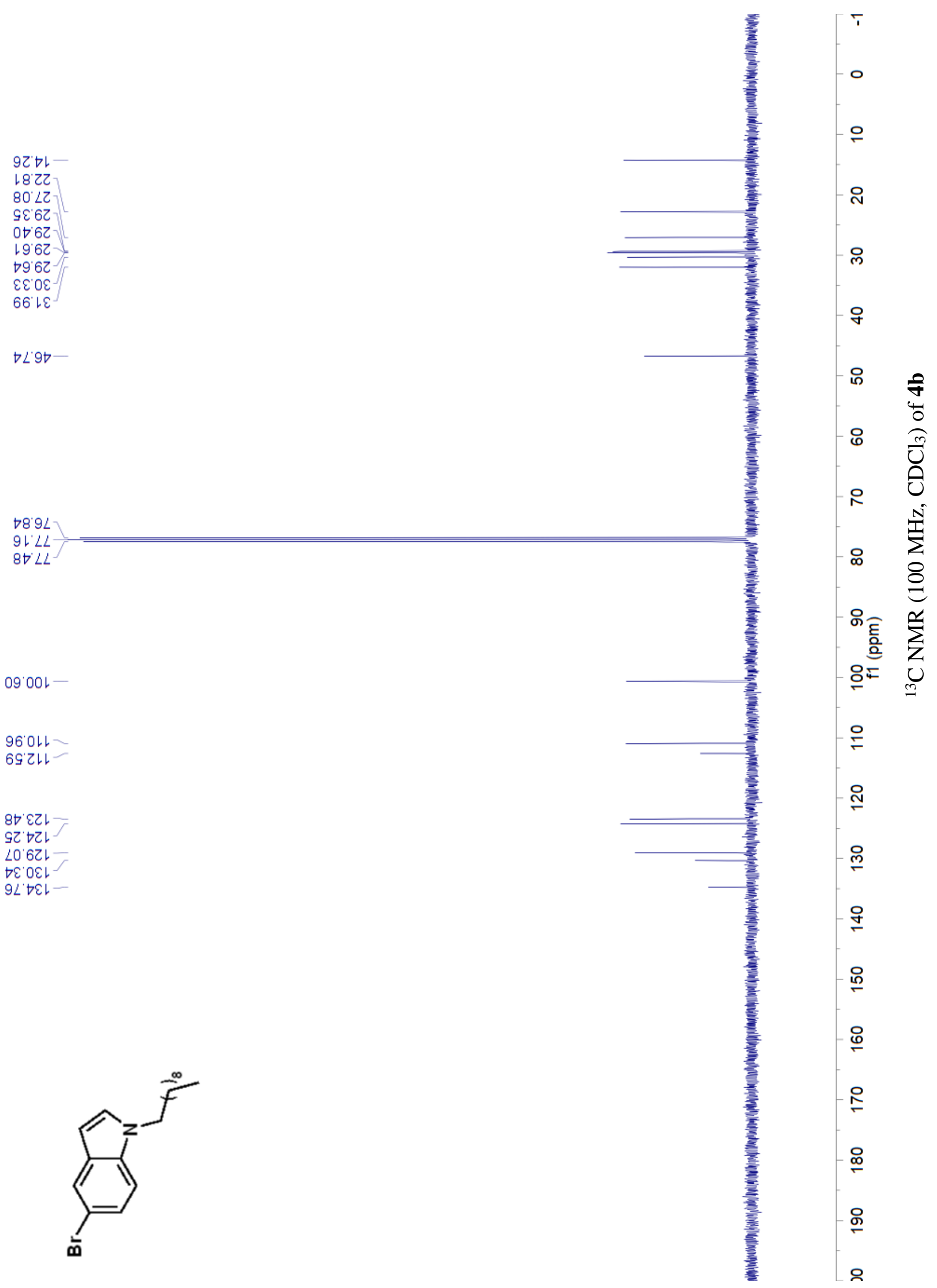

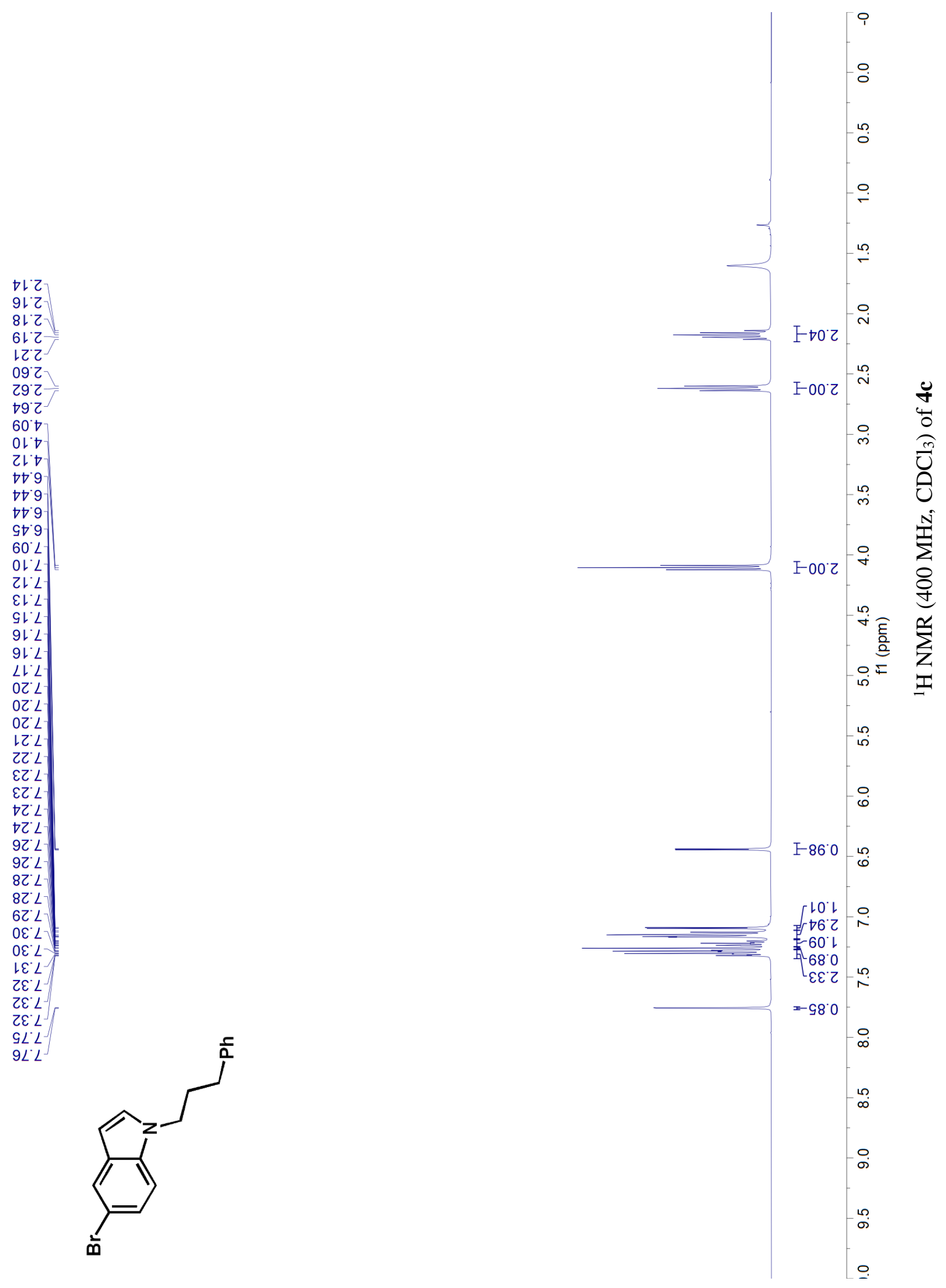
$\nabla S^{\prime} L \mathcal{E}$
$86^{\prime} Z \mathcal{E}$

$6895-$

$\nabla 89 L$

8DLL

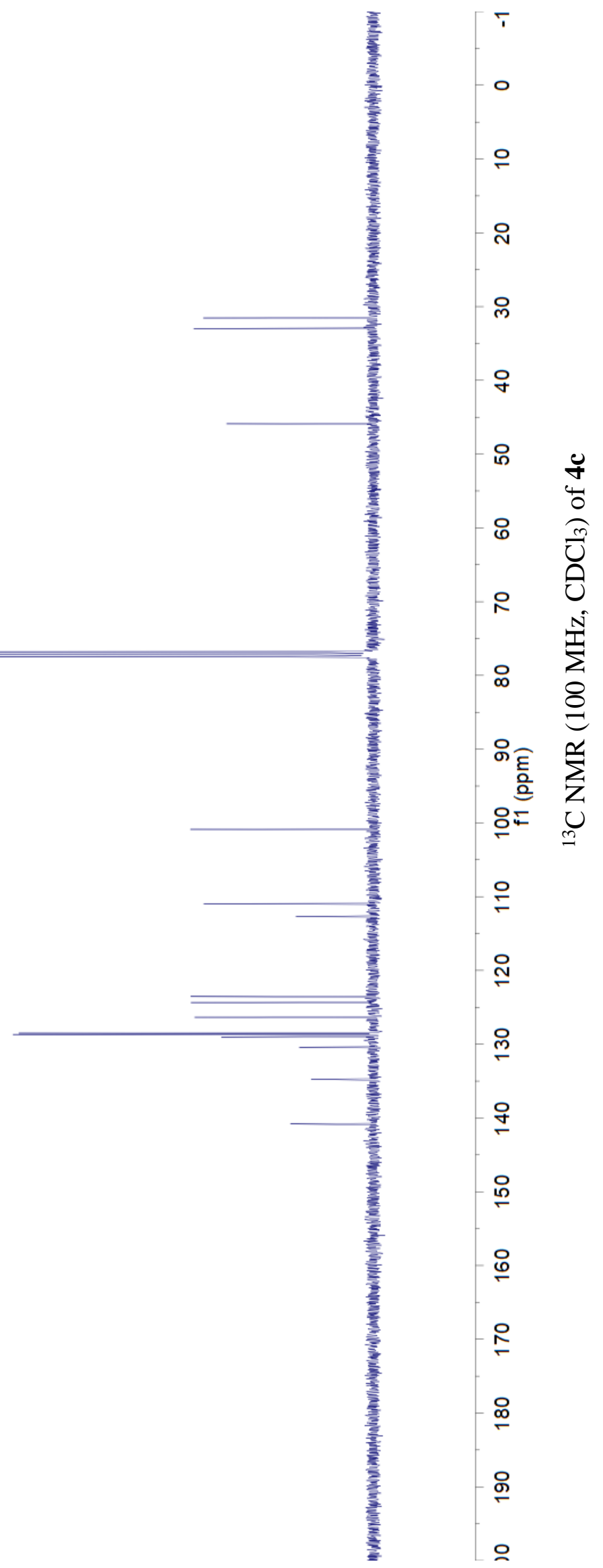

$\angle 8^{\circ} 00 \mathrm{~L}$

S60ll

LLZL

$\nabla S^{\prime} \varepsilon Z \downarrow$

SE' $\nabla Z L$

sE'9ZL

LG'8ZL

89'8Z

$0062 \mathrm{l}$

$6 \varepsilon^{\prime} 0 \varepsilon$

$G \angle \nabla \varepsilon L$

Z8'0t

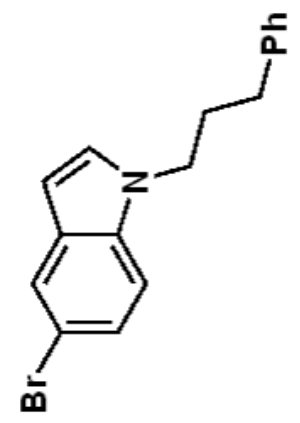



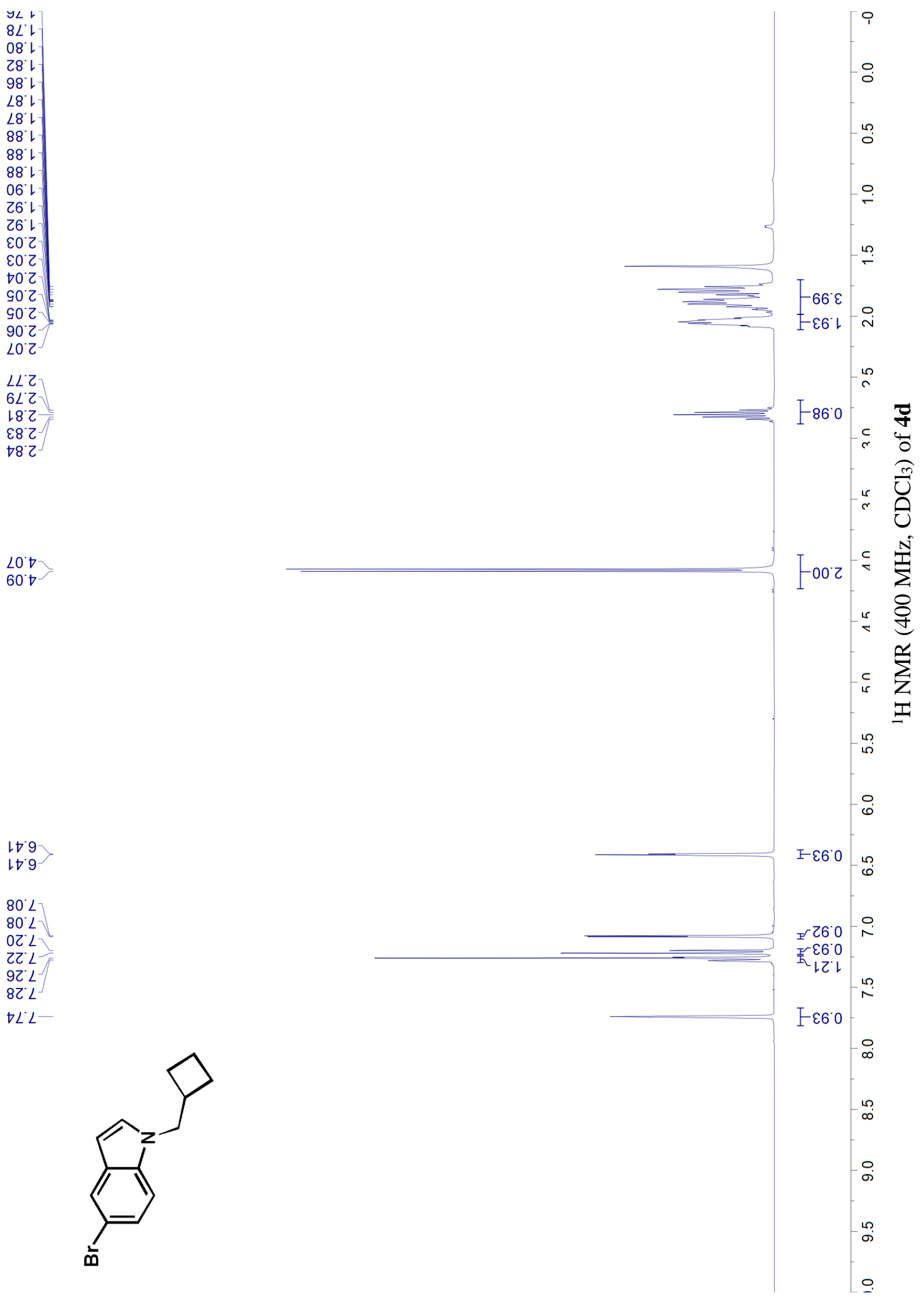


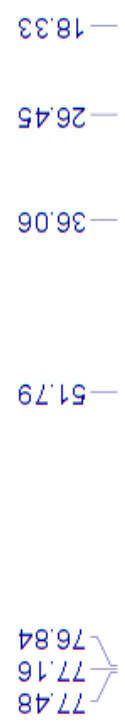

$89^{\prime} 00 \mathrm{~L}$

เoルレ 6Sてル-

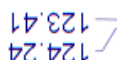

so' 6 b ZZ'OहL

$86^{\circ} \triangleright \varepsilon\llcorner$

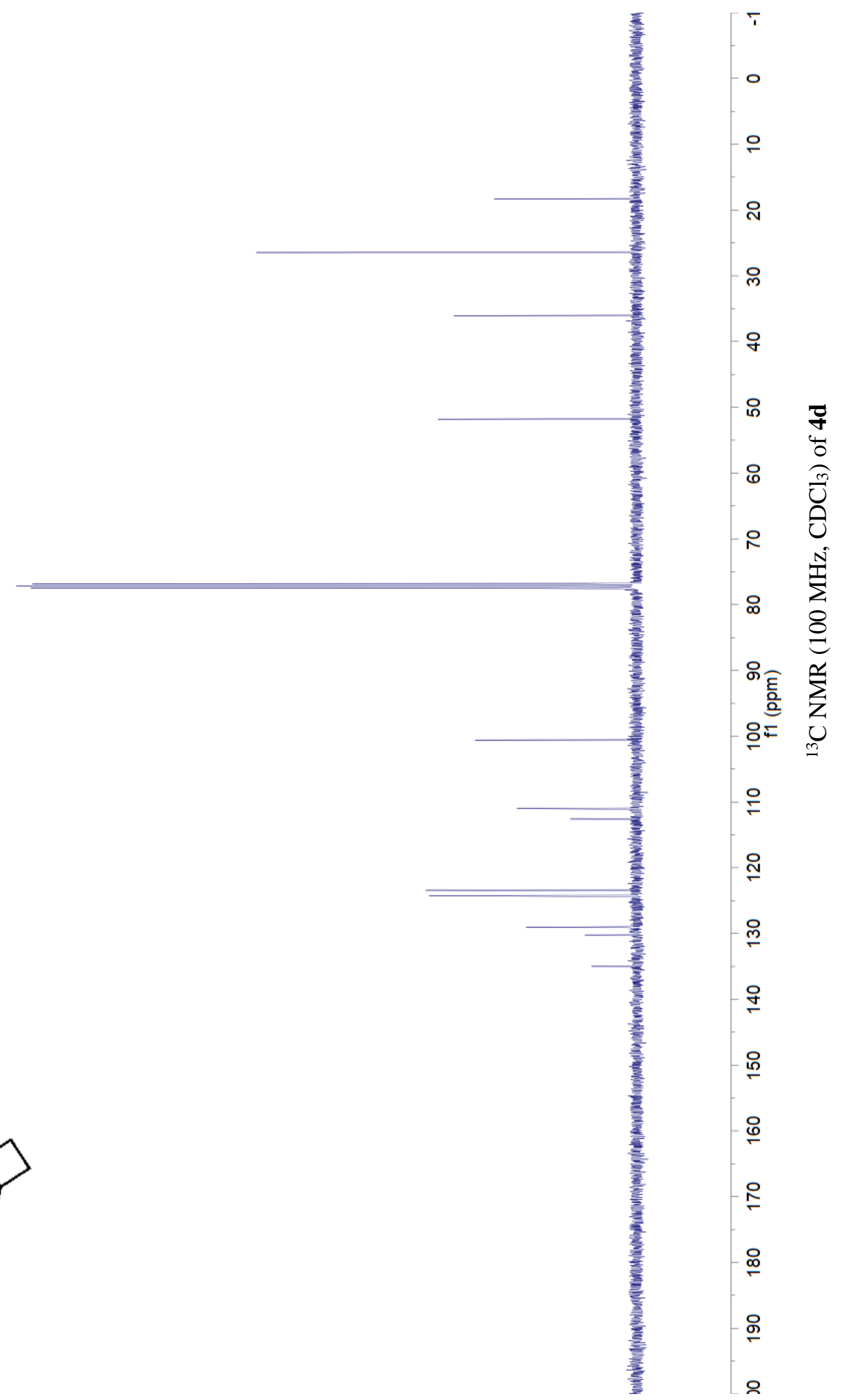



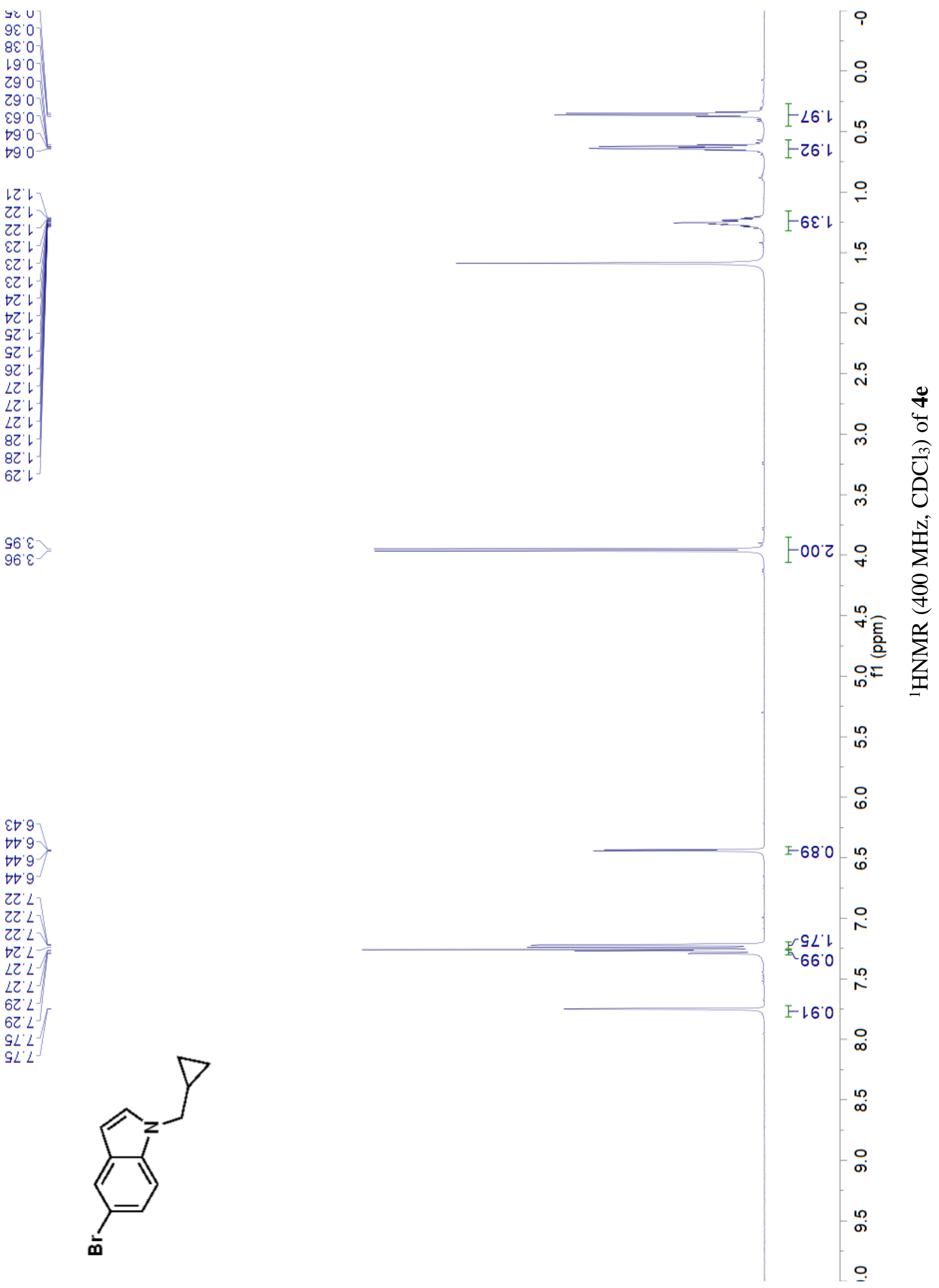

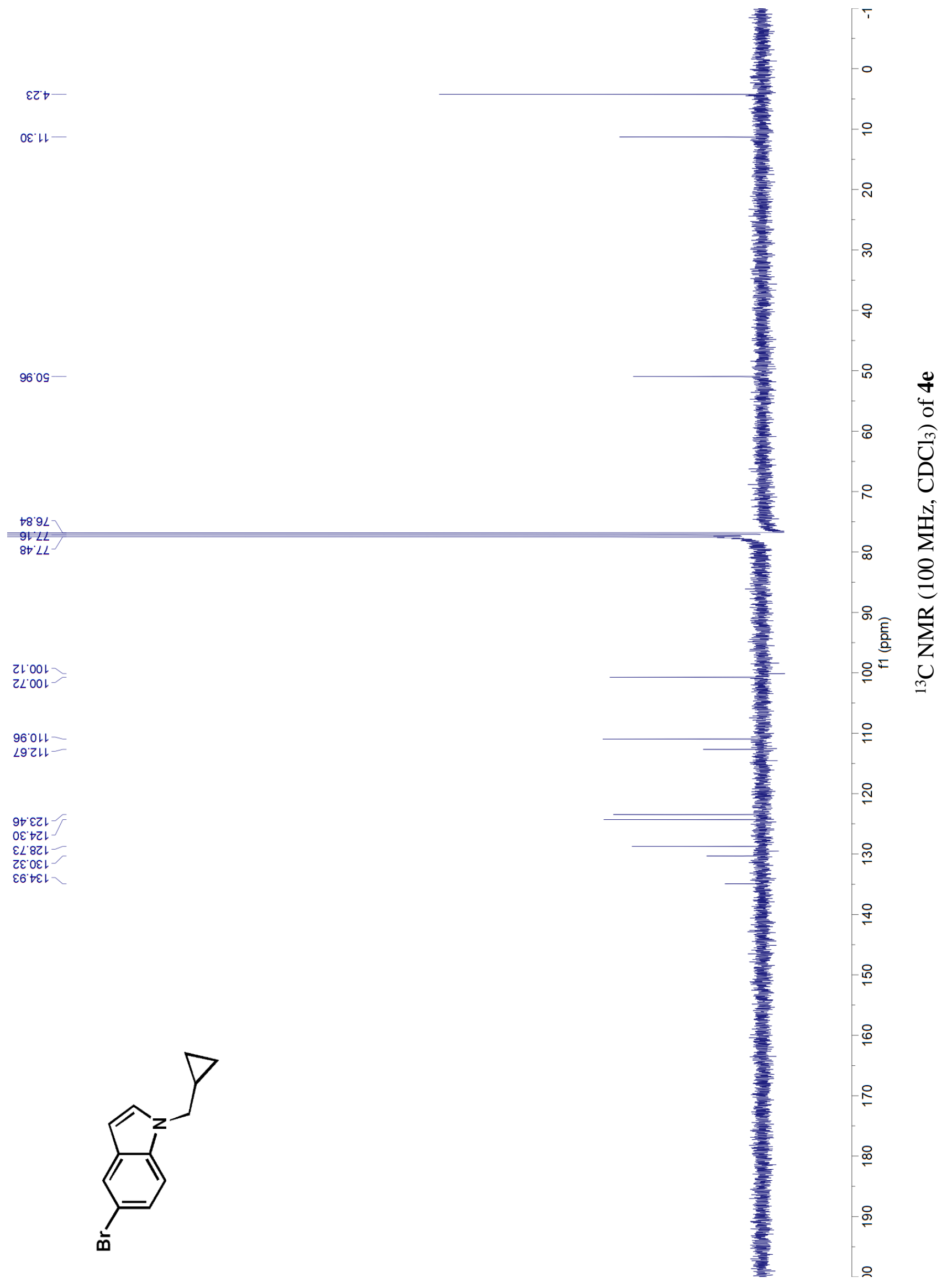


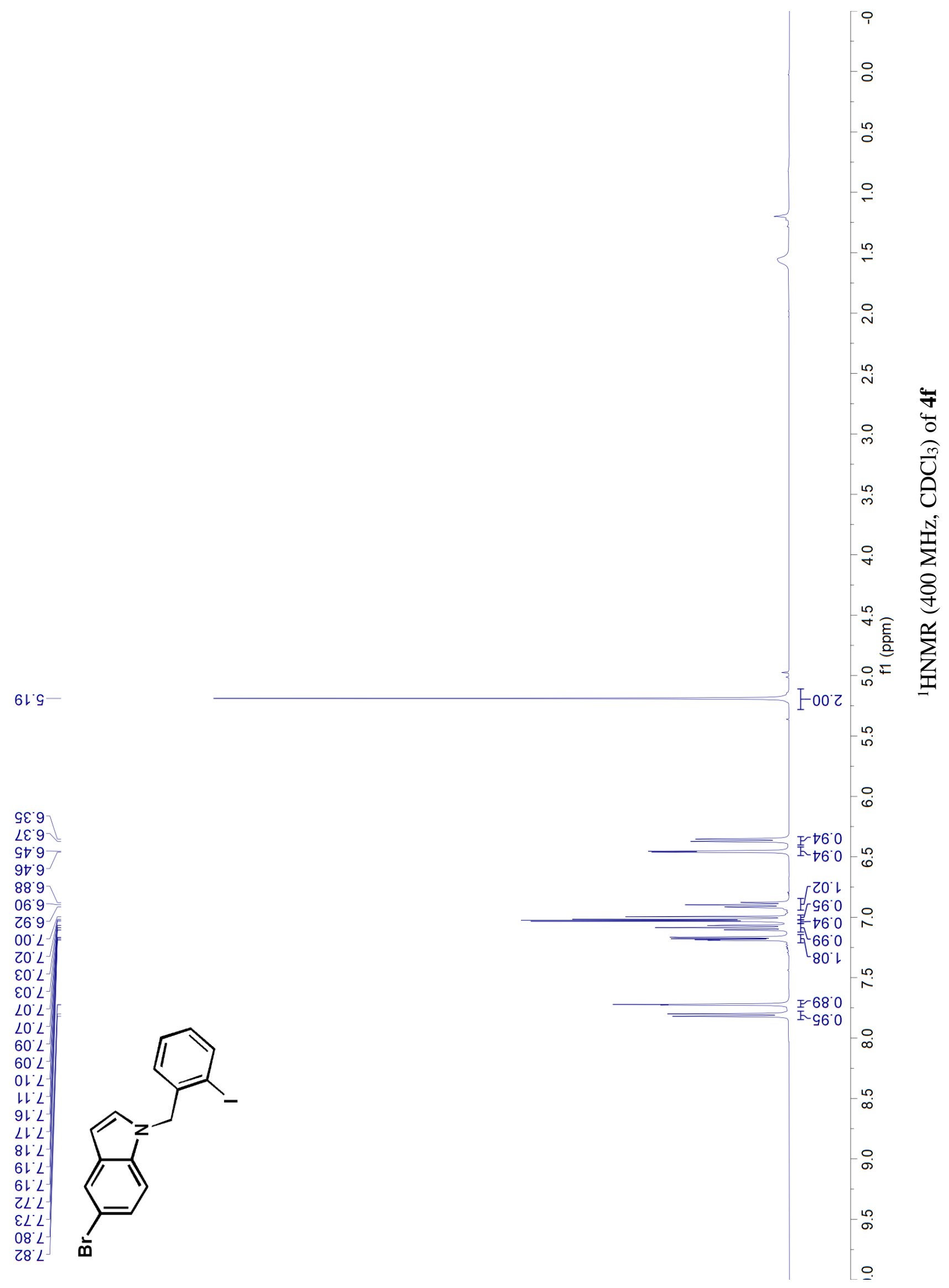


$6 \mathcal{G} 9 \varsigma$

$789 L$

GL'LL

$\angle \nabla^{\circ} L L$

$92 \angle 6$

†8เ゚レ-

$\nabla \varepsilon$ レレ

૬でยレ-

$99^{\circ} \varepsilon 乙 レ$

$06{ }^{\circ} \nabla Z L$

69 $\mathrm{LCL}$

†8 8टL

99.6己ᄂ

ह७ OEL

LO $G E$ L

$60^{\circ} 6 \varepsilon \mathrm{L}$

ह9.6 6 ᄂ
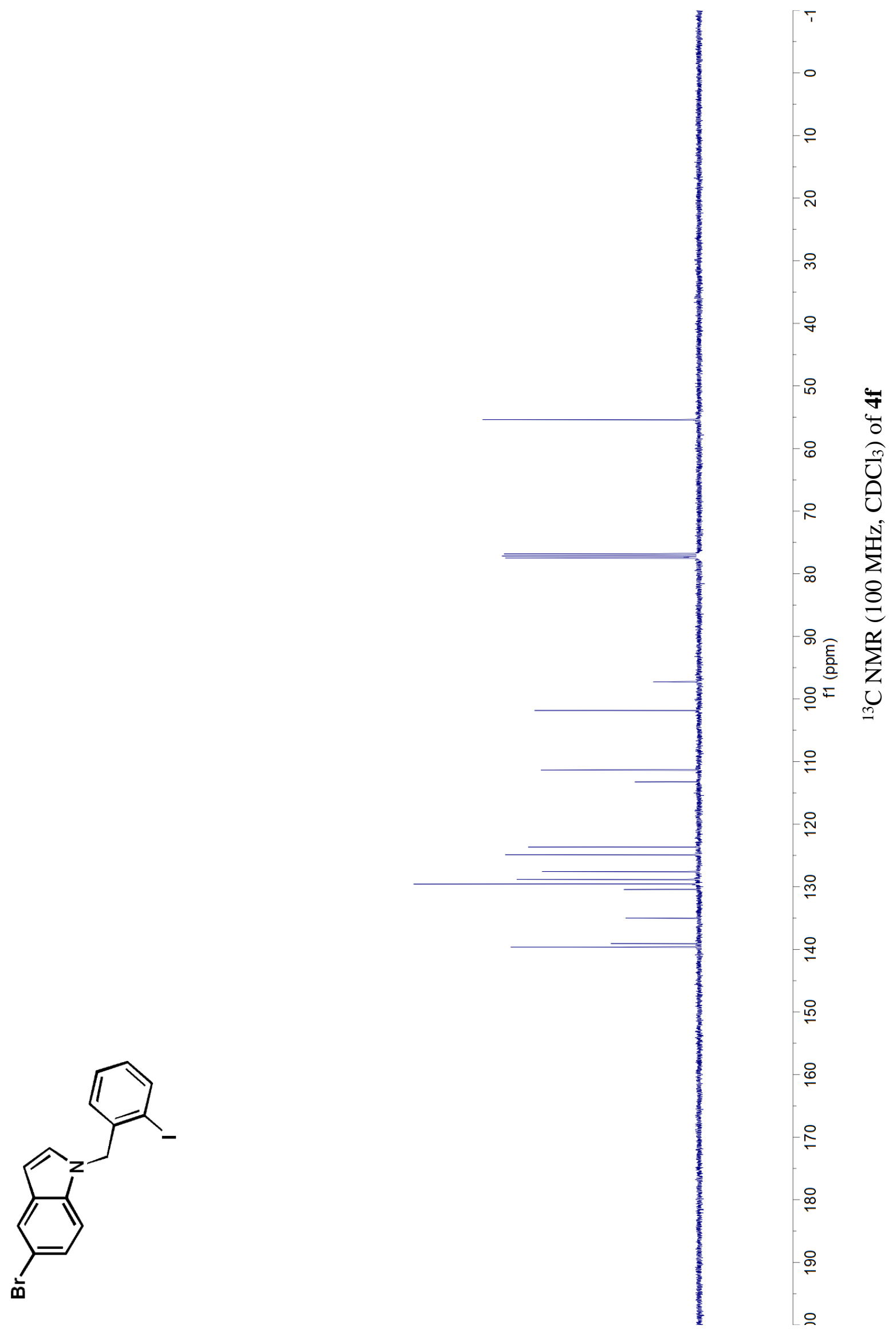


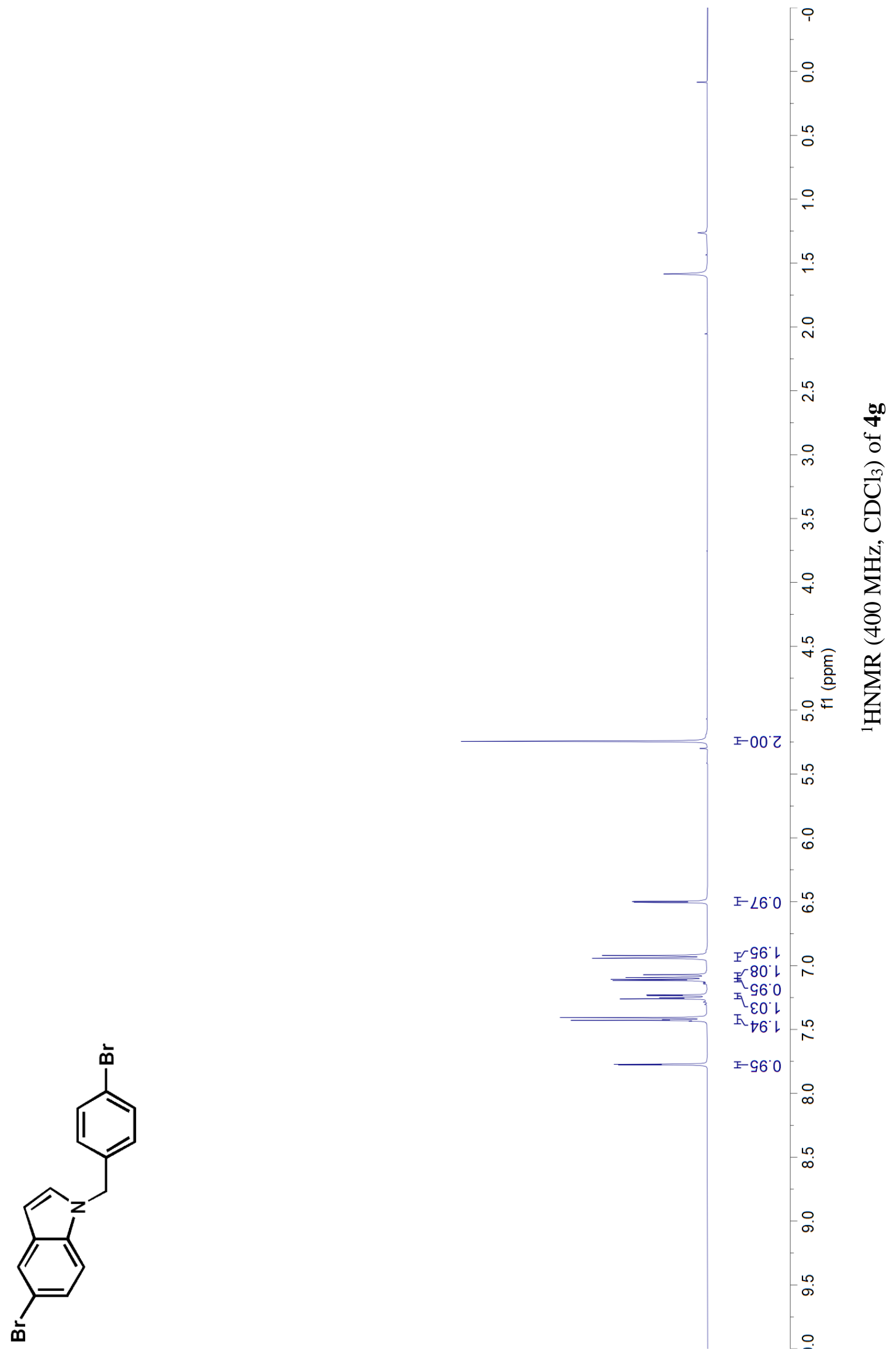



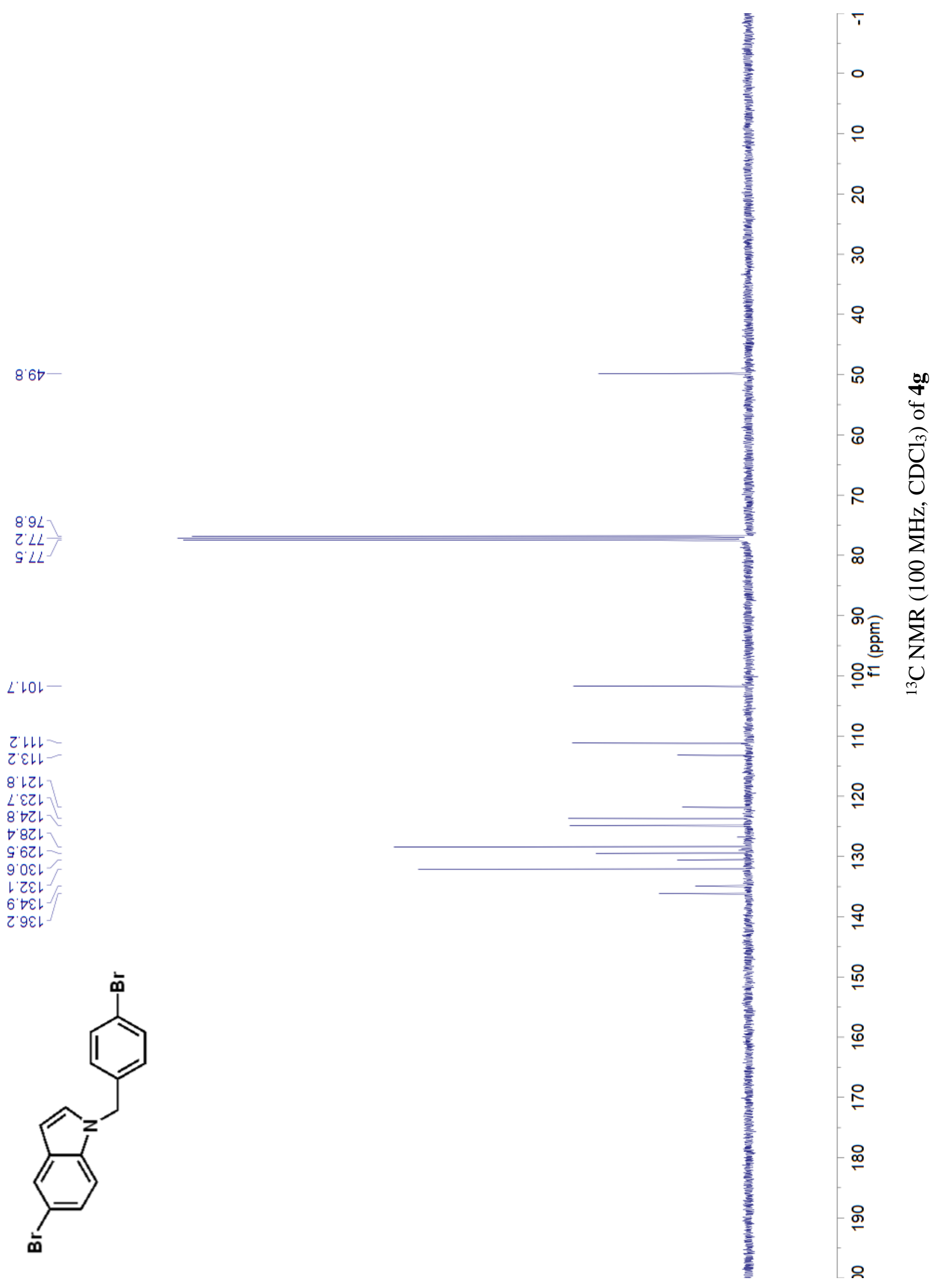

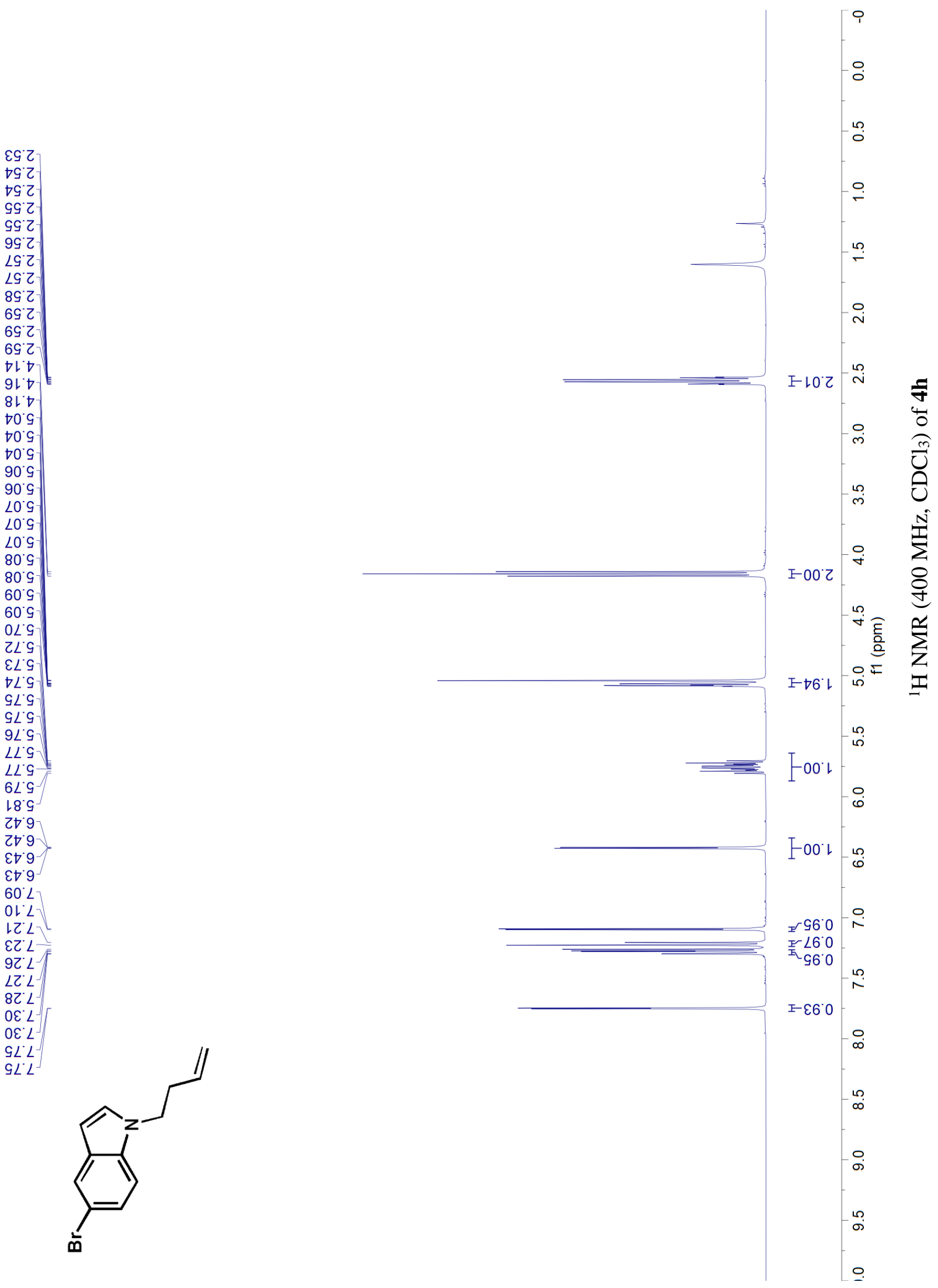
$\downarrow S \downarrow \varepsilon-$

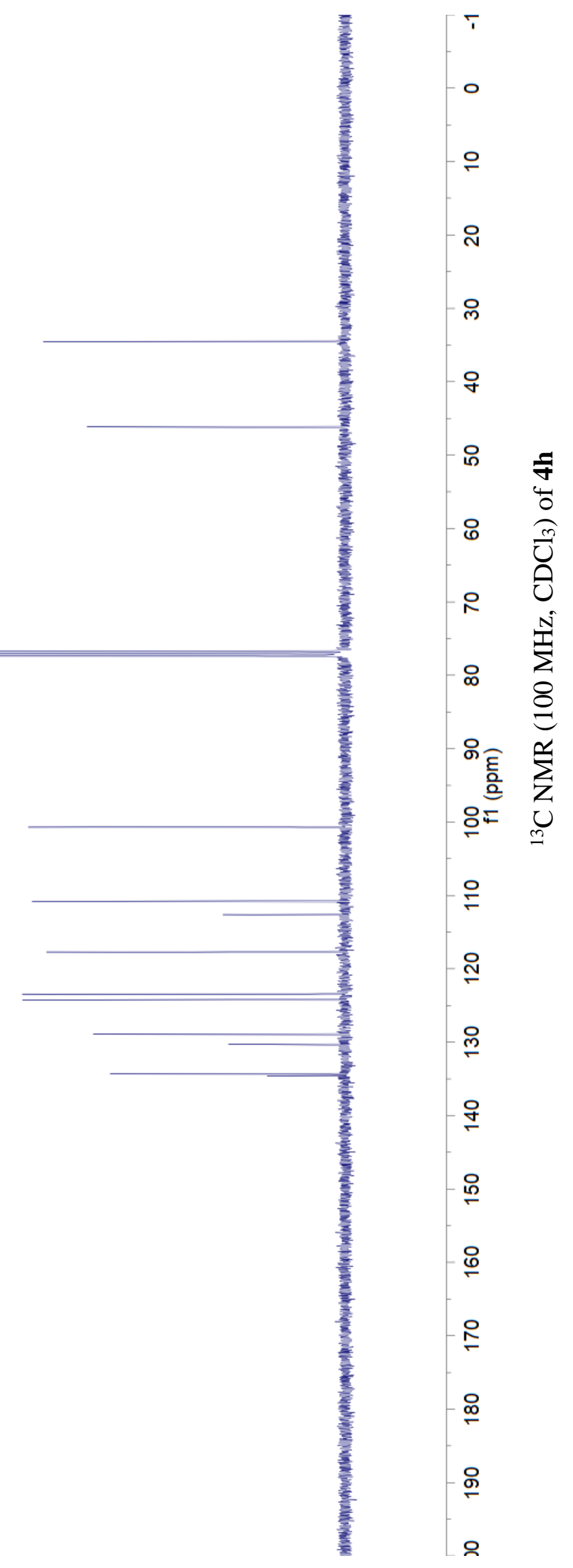

SOLL

$S O L L$
$\angle E^{\prime} L L$

OLOOL-

28016

I9ZLL

LLLLL

$\varepsilon \nabla^{\prime} \varepsilon Z L$

$G Z ' \nabla Z L$

\&6'8ZL

$8 Z^{\circ} 0 \varepsilon \downarrow$

se' $\forall \varepsilon$

$8 S^{\prime} \forall \varepsilon L$ -

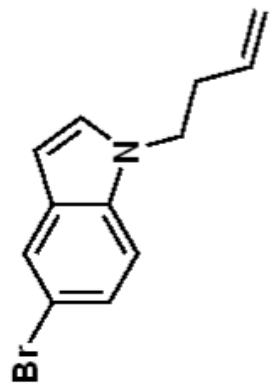




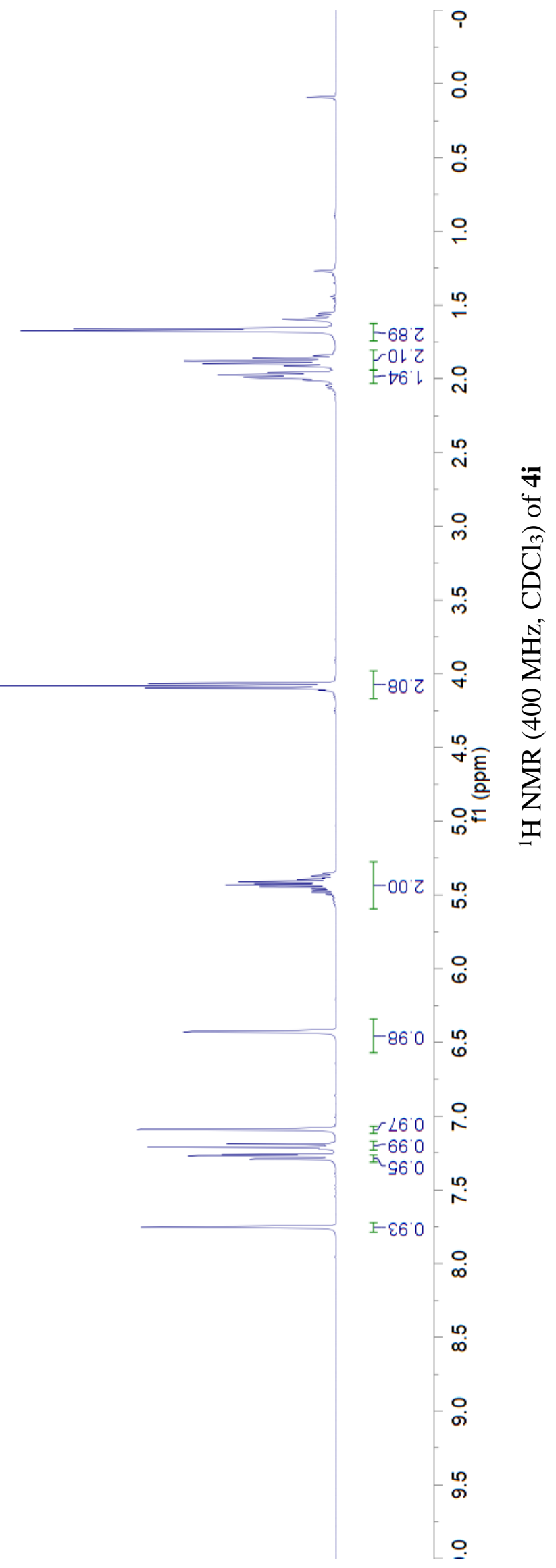




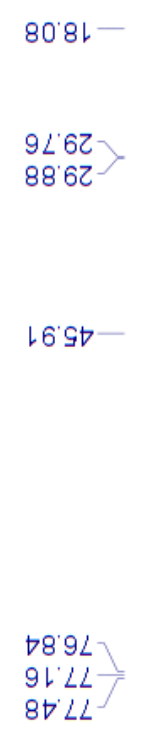

$99^{\prime} 001-$

66아

เ9己レ

Lจ E己L

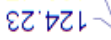

ZG'9ZL-

LO'6Zl-

L $\angle$ 6Z L

૬ع'0ع -

$S L \nabla \varepsilon L$

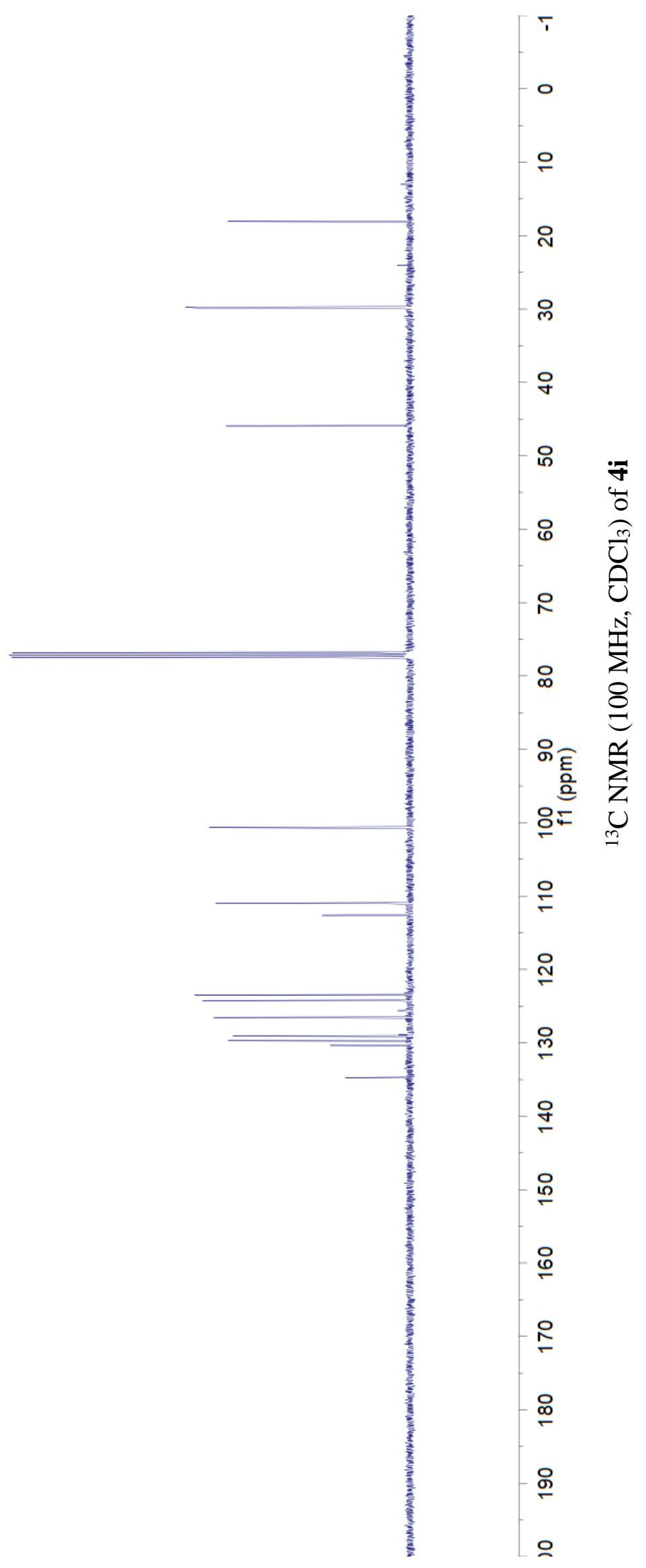




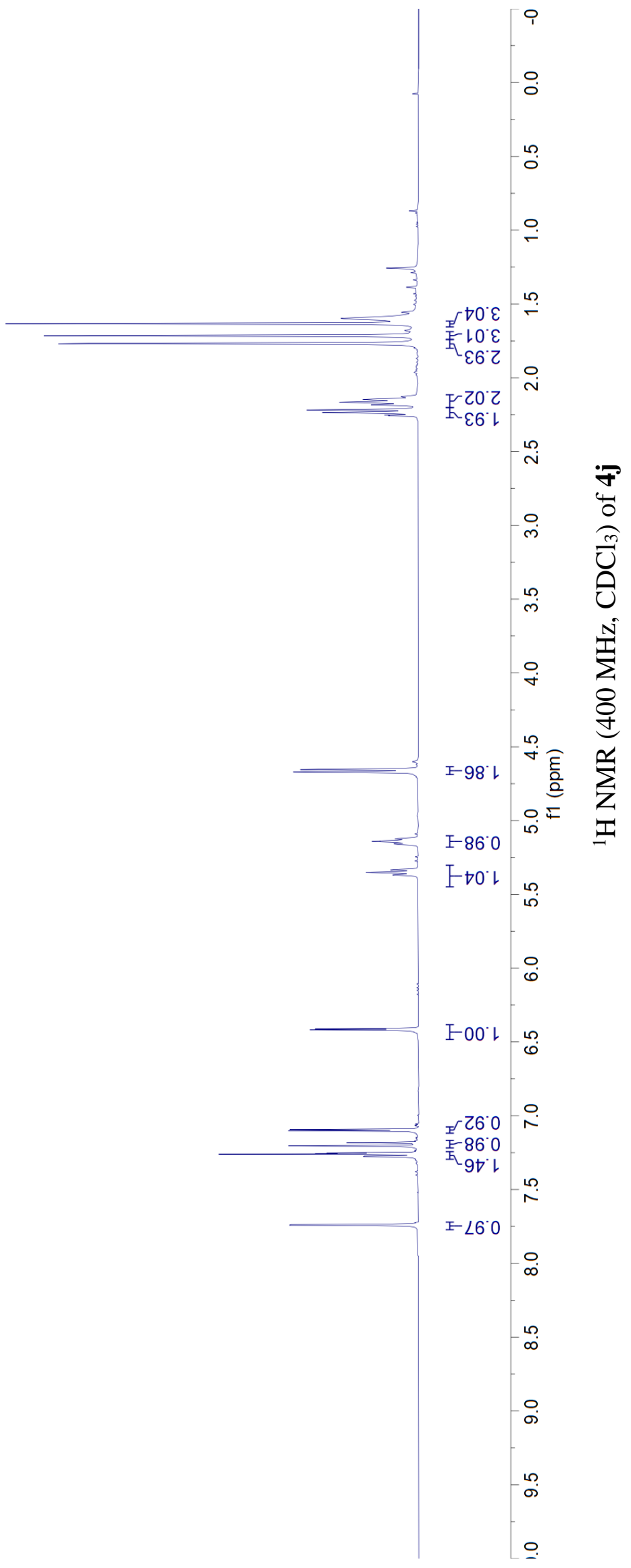


$\angle 8 \angle L$

เナとZ

$\varepsilon 6.92$

OS' $9 \mathrm{Z}$

เฉ $Z \varepsilon-$

6 เトtー

$\nabla 89 L$

$91 L L J$
$87 L L$

$\angle 9^{\circ} 00 L^{-}$

60ルル

OLZL

$\varepsilon \varepsilon^{\prime} 0 Z$ L

ह† EZL

09 'ยटᄂ

$\varepsilon Z^{\prime} \nabla Z L$

$\angle 9 ' 8 Z 1$

$670 \varepsilon$

L'ZEL

$\nabla L \nabla \varepsilon L$

与E'ODL

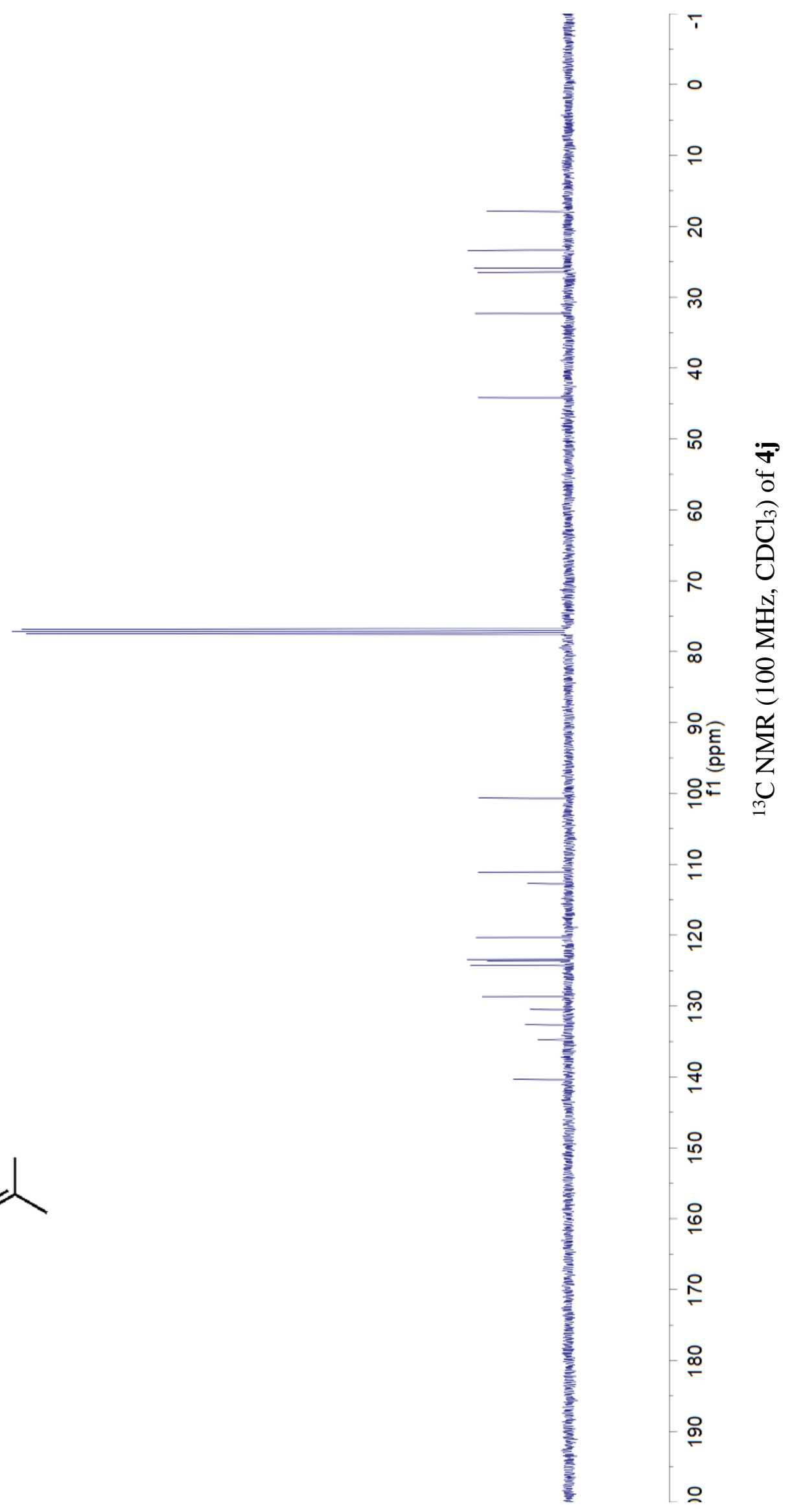


乙†

$\varepsilon 8^{\circ}$ L

G $8^{\circ}$ -

$\angle 8^{\circ}$.

$68^{\circ} \mathrm{L}$

$16 \mathrm{~L}$

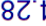

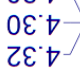
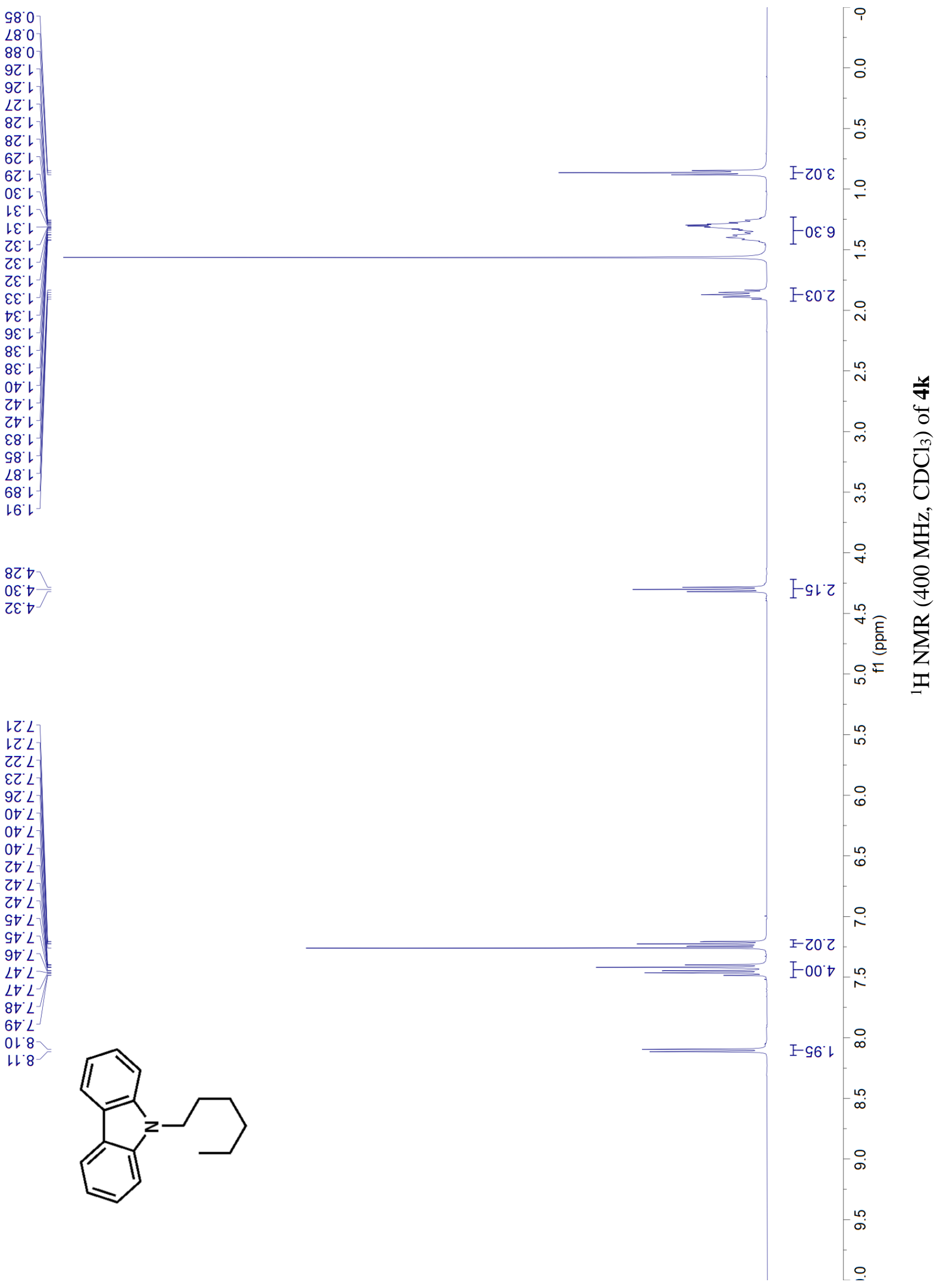
$92 \angle$

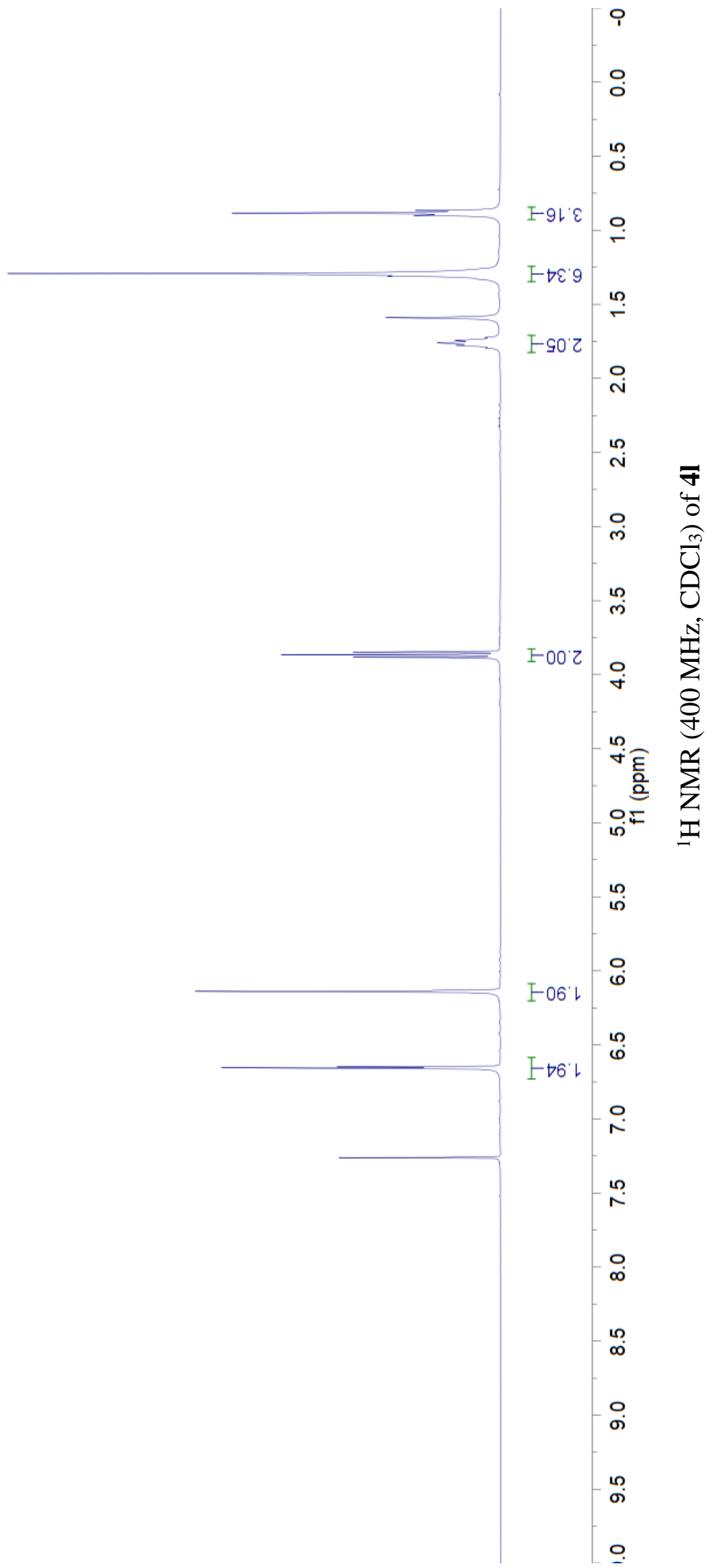


9ト・レー

$\angle 9 Z^{\circ}$

6992

$\nabla S L E$

$69 \cdot \varepsilon$

$\nabla 8^{\prime} 9 L$

$9 L: L L$

$8 \nabla L L$

S8 $\mathrm{LOL}-$

0902

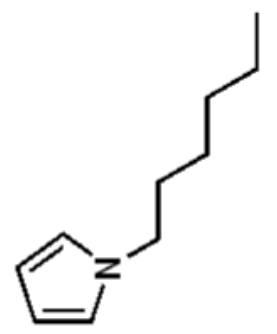

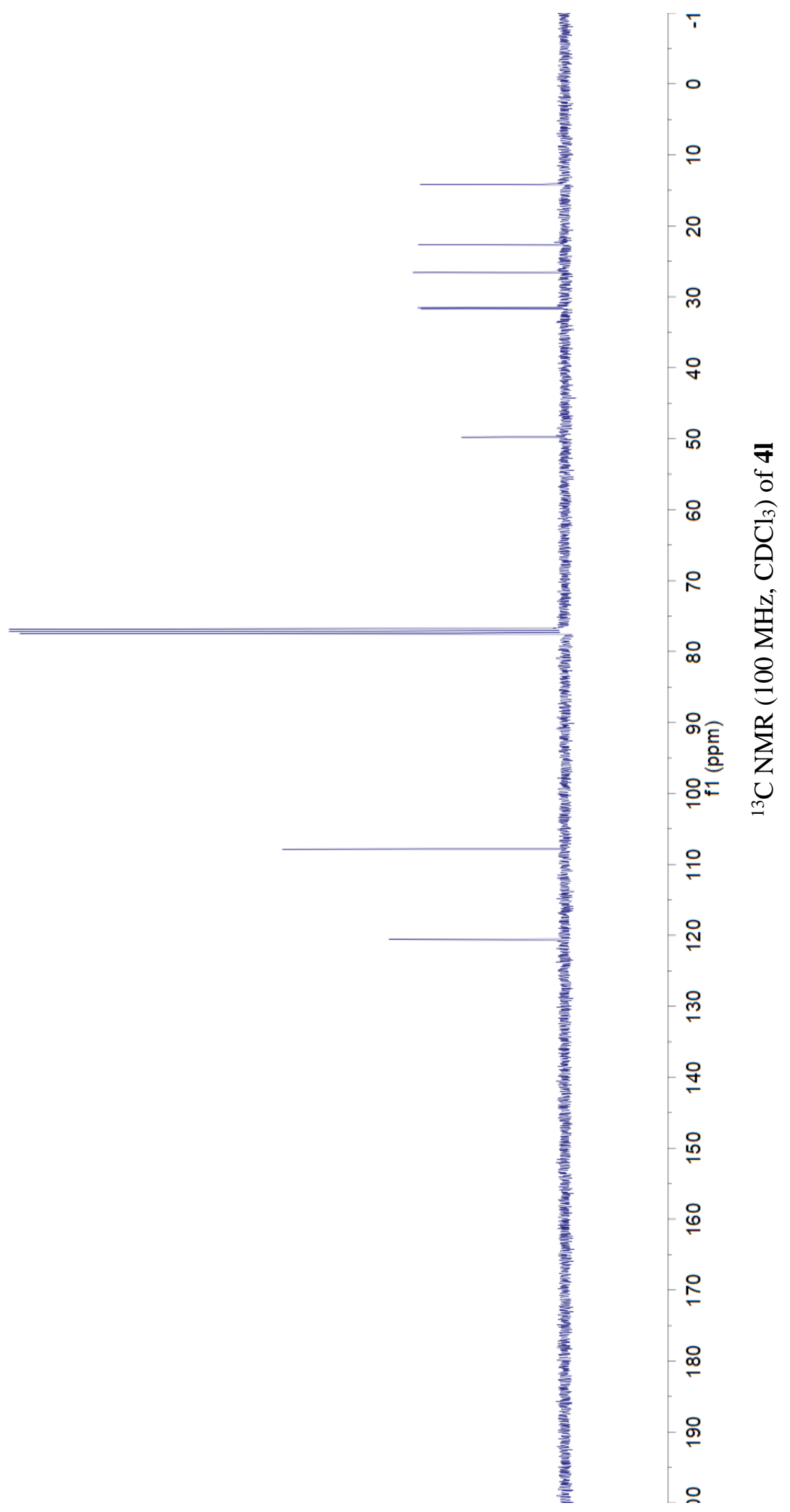

SI-96 
78 -

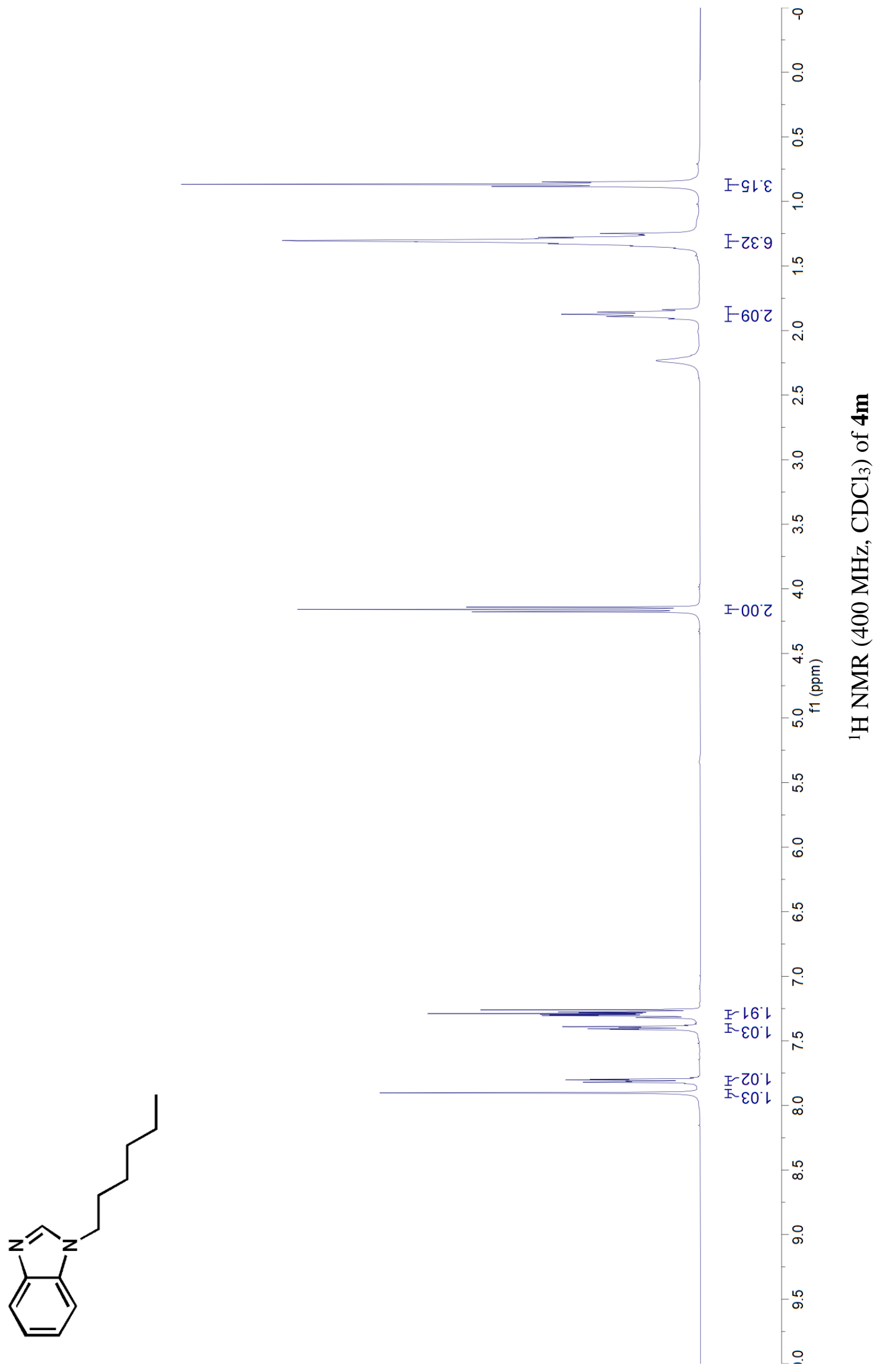




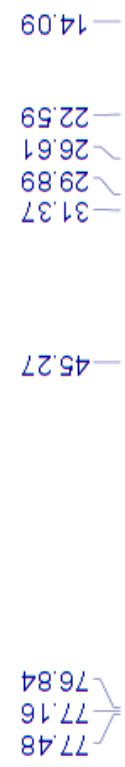<smiles>CCCCCCCCCC=Cc1ccccc1</smiles>

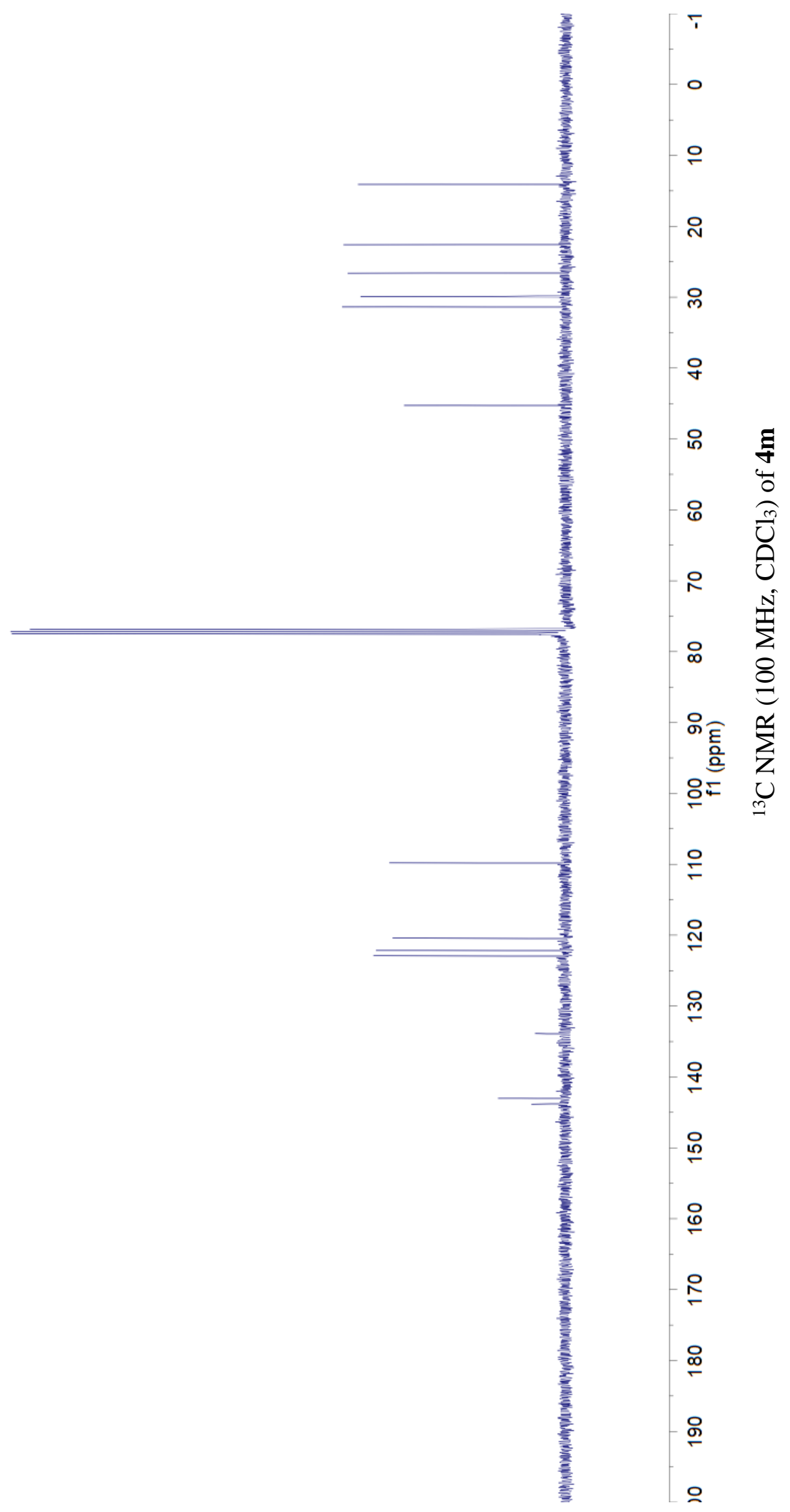




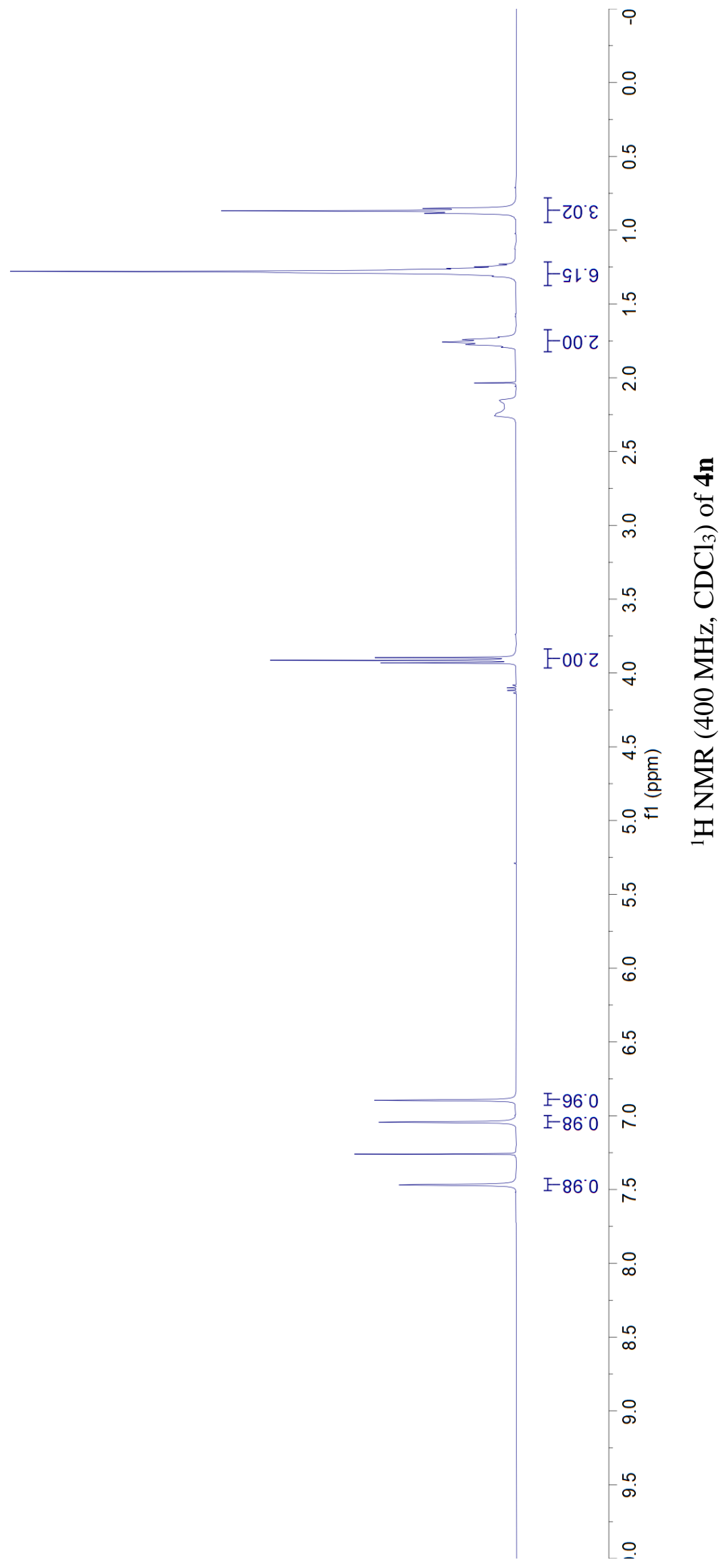

$06.9-$

$-96^{\circ} 0$

$\triangle O^{\circ} L$

$\angle D L-$

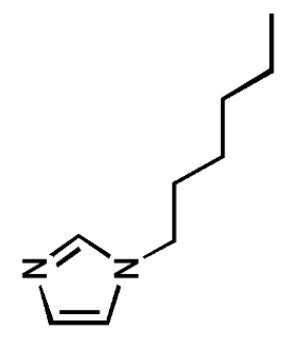



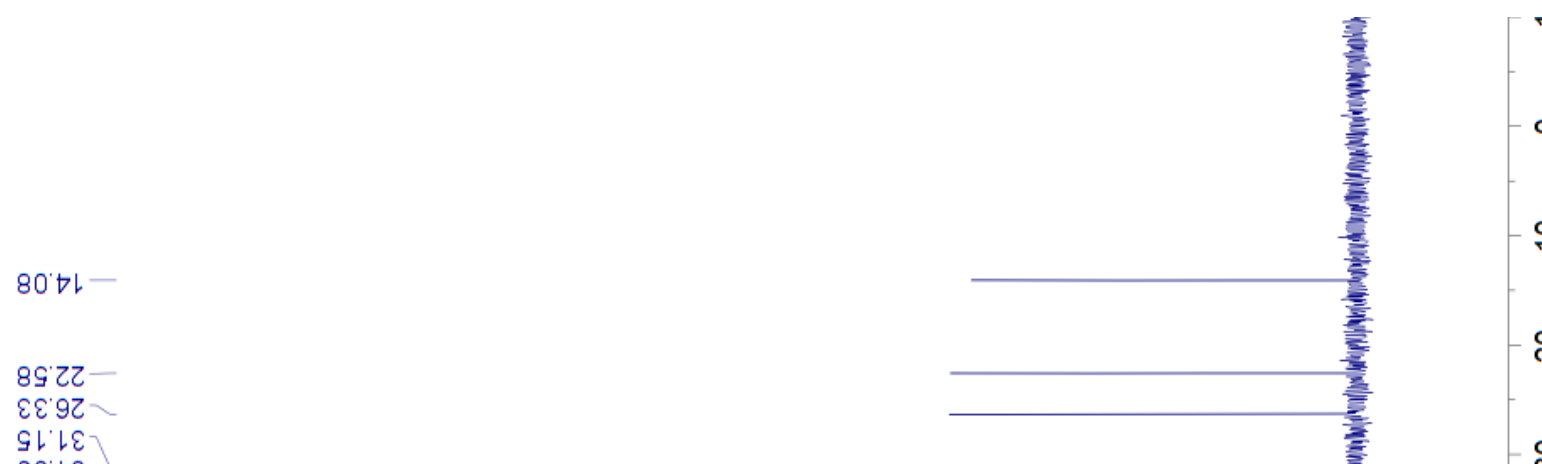

$9 L L L$
$8 \nabla L L$

2681 -

เย'6てレ-

$\nabla L \cdot \angle \varepsilon L-$
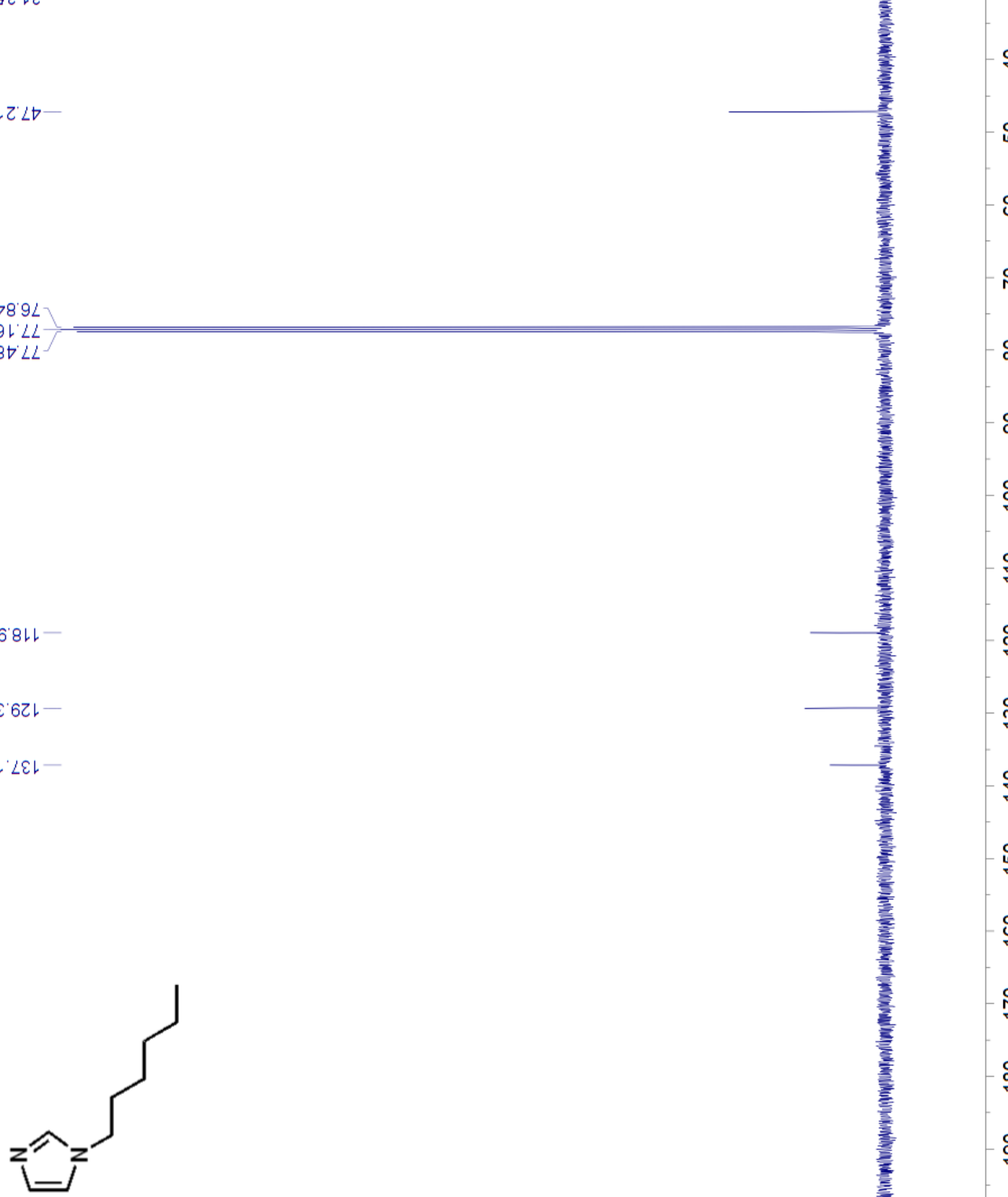

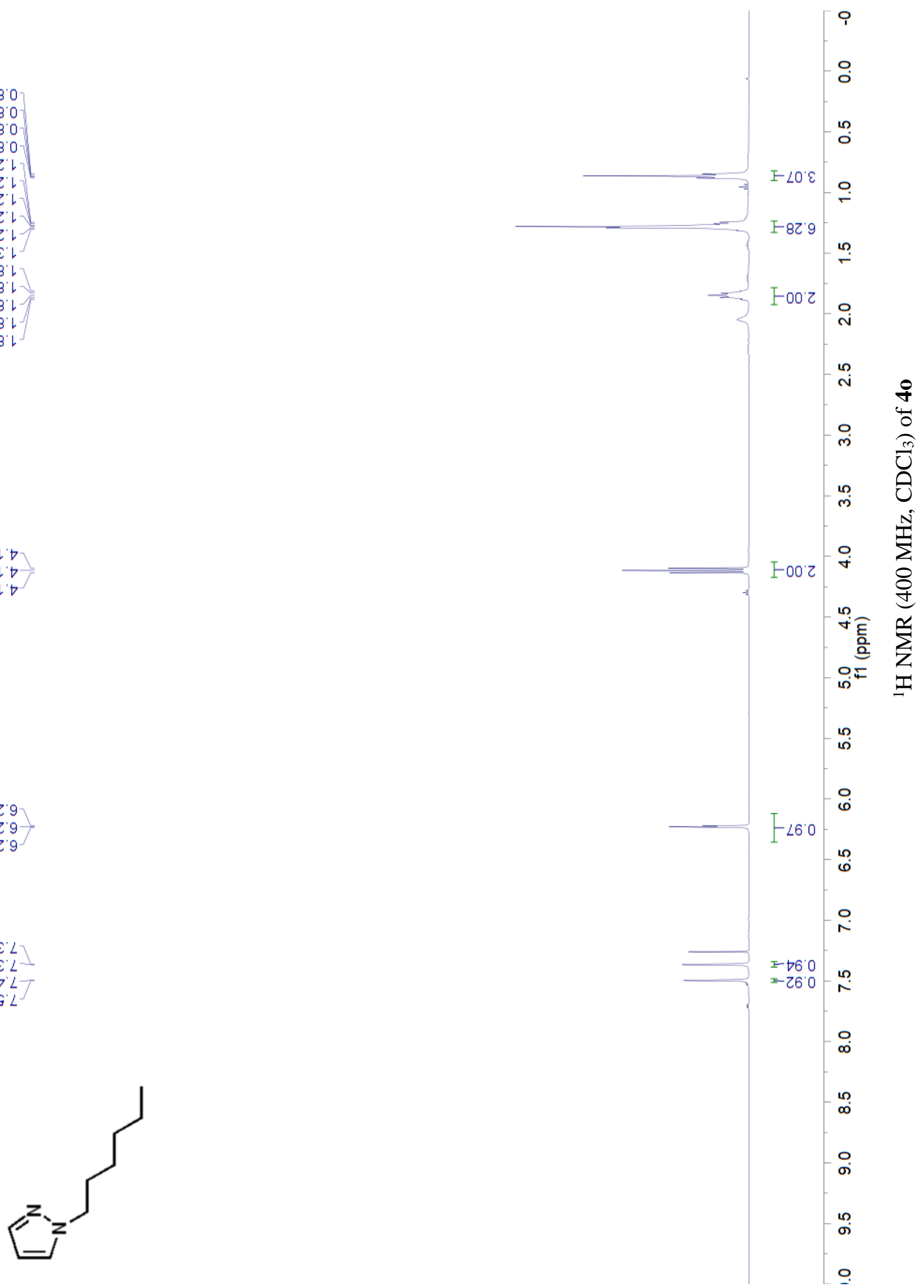
レナレー

$$
\begin{aligned}
& \begin{array}{l}
1922- \\
1792-
\end{array} \\
& \nabla G^{\prime} 0 \varepsilon-
\end{aligned}
$$

8ट $29-$

$\nabla 89 L$

8DLL

9Z SOL-

96.8Zレ-

$90^{\circ} 6 \varepsilon\llcorner$

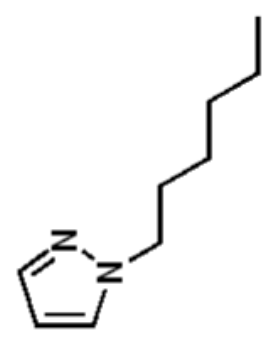

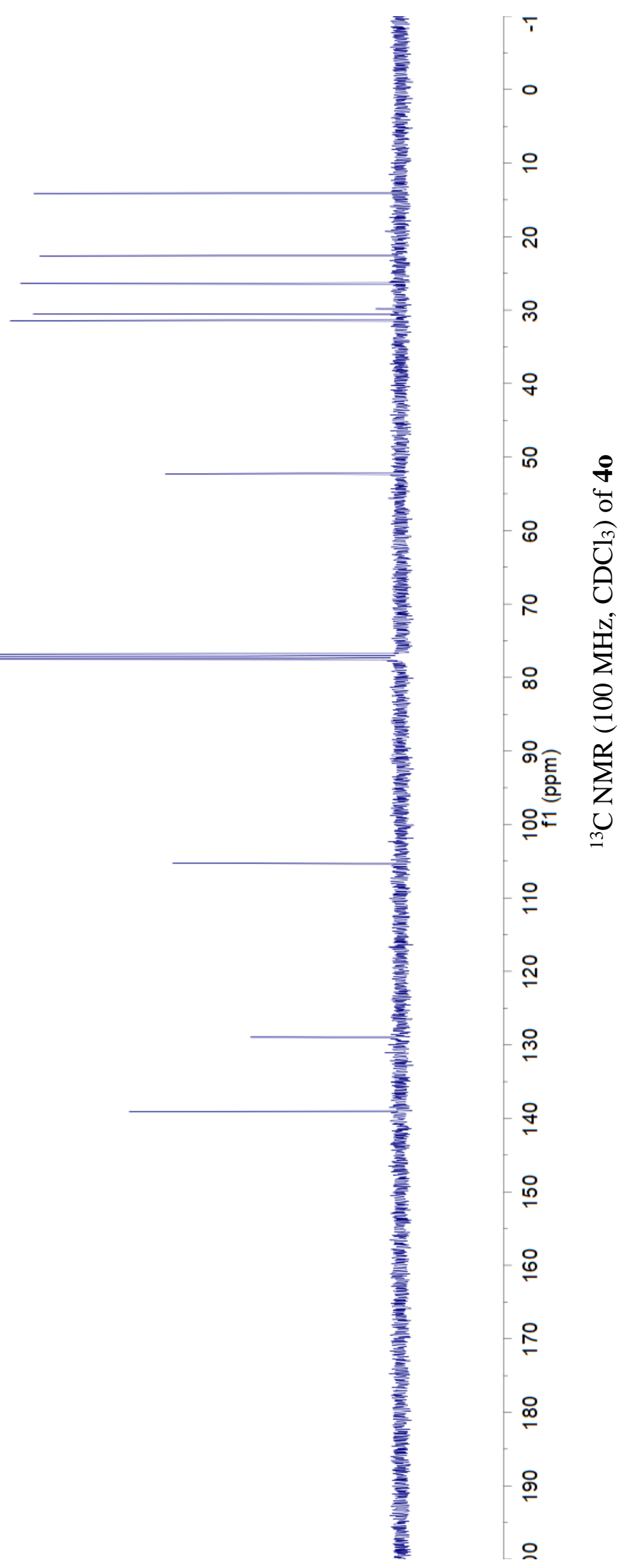



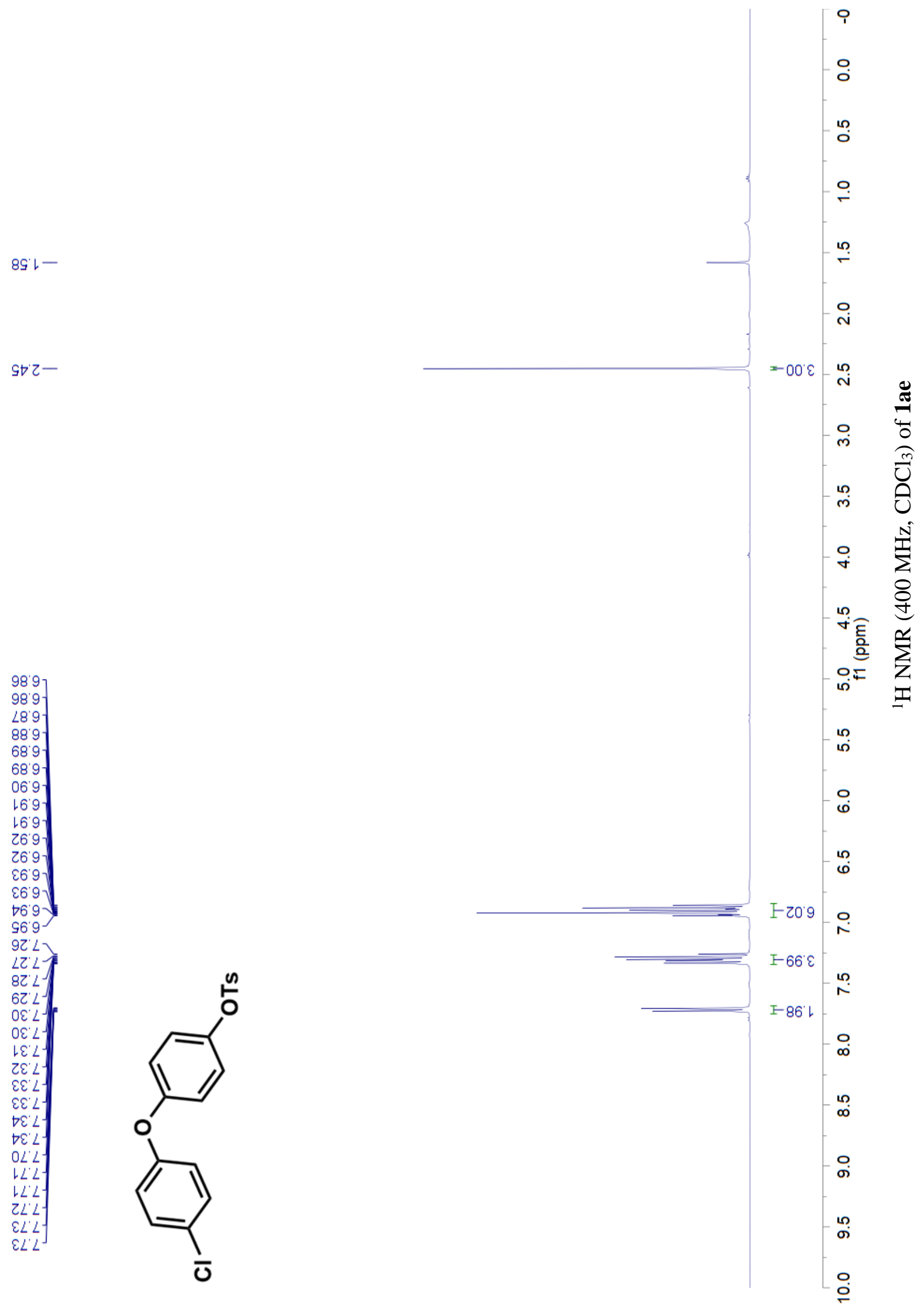
68レー

$789 L$

$91 \angle L$

$8 \nabla^{\circ} L L$

เt6เL

OG.0ZL

L6'EZL

$\angle 98 \mathrm{CL}$

$1062 \mathrm{~L}$

26.62L-

LO०EL

เย'てยเ
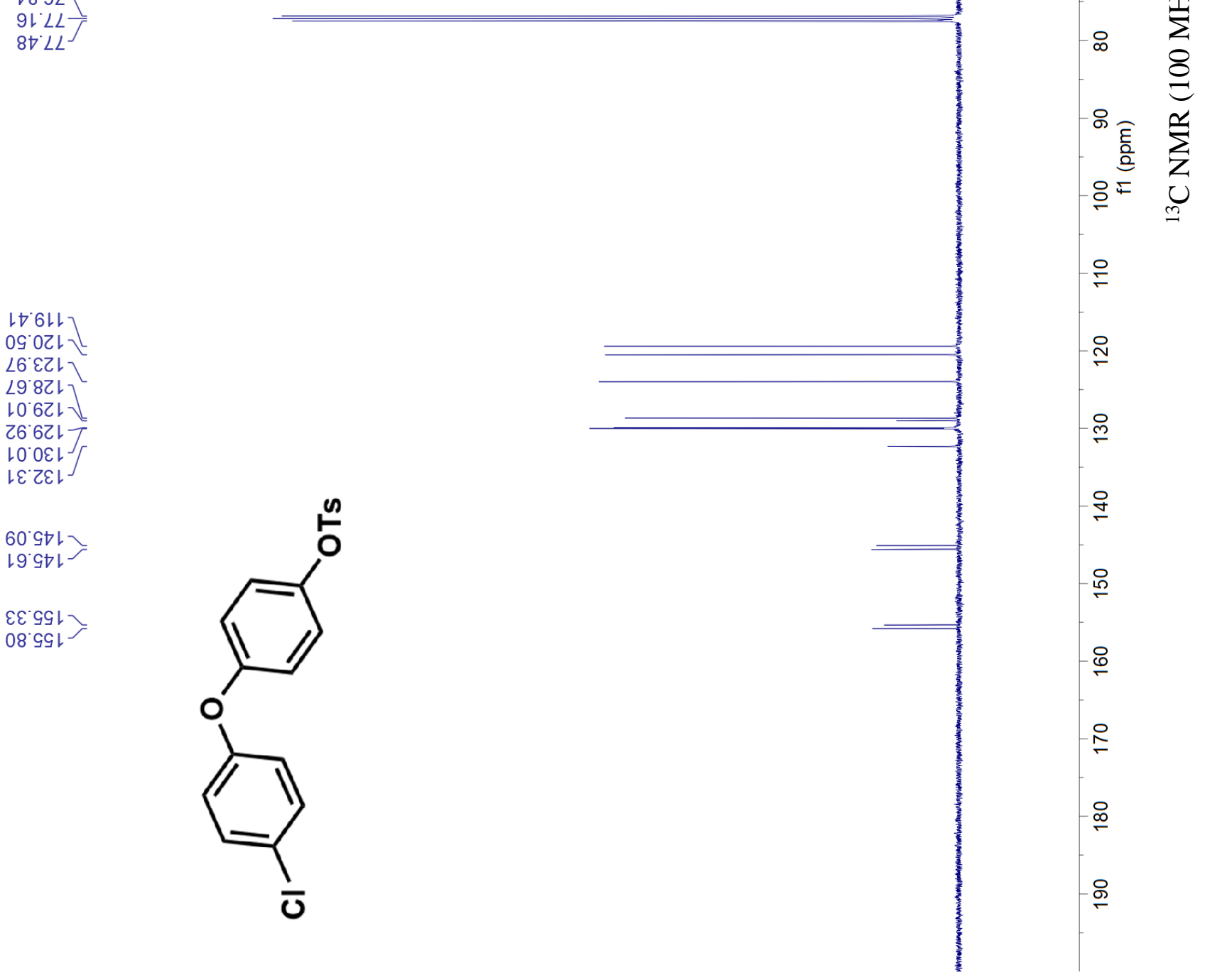

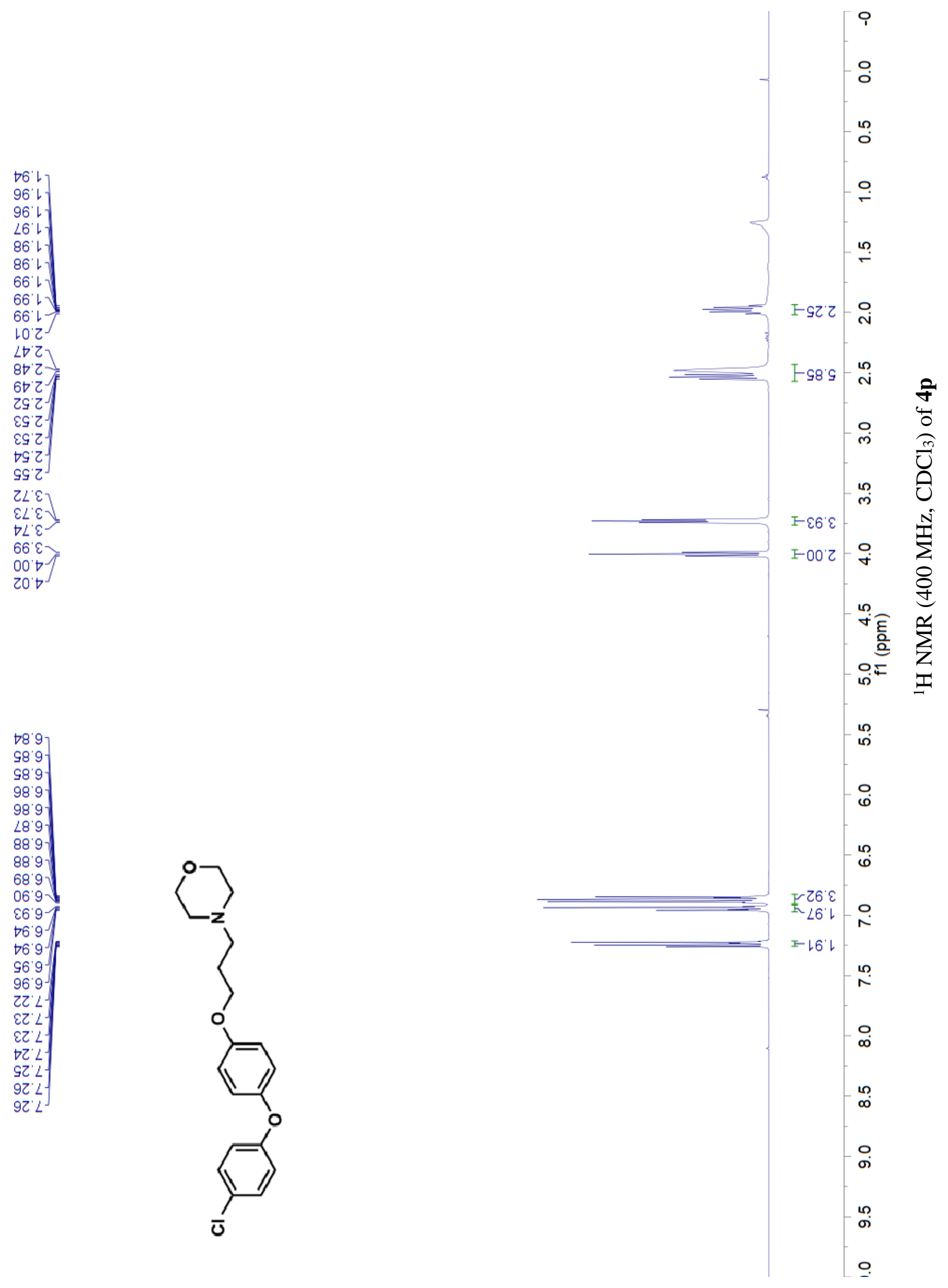
$\angle 9^{\circ} 92-$

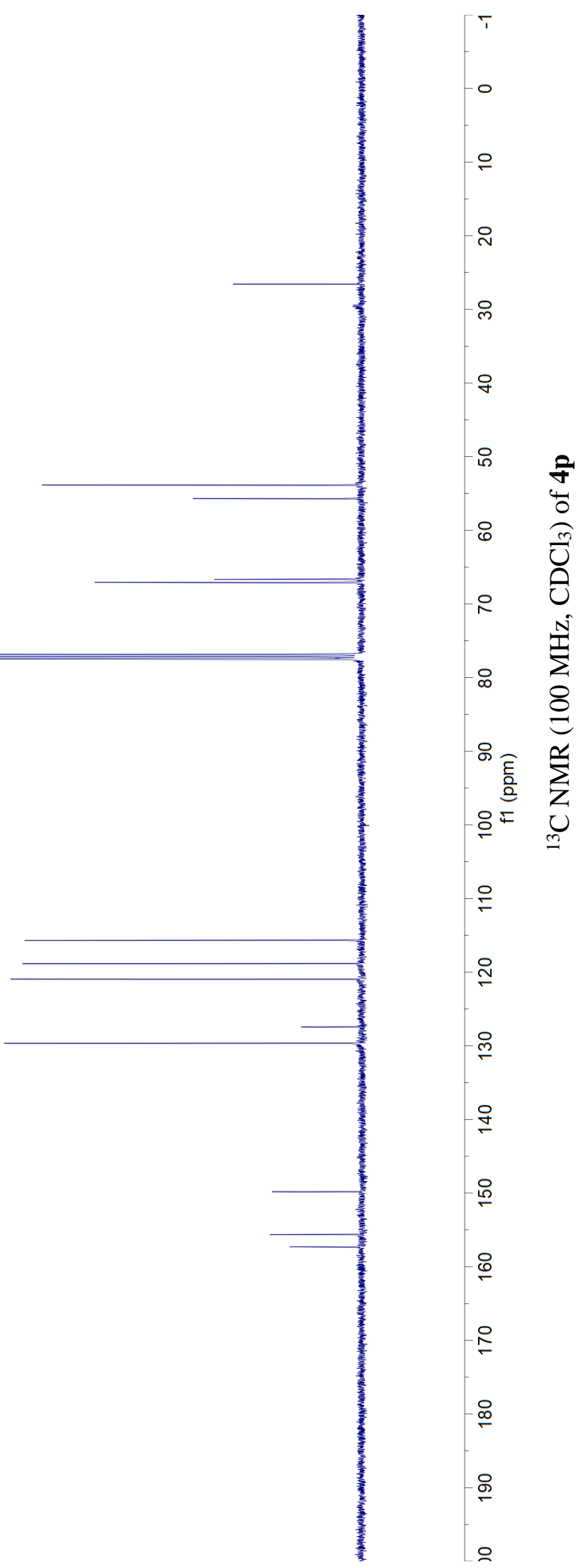

89.5 -

98. $8 \mathrm{ll}-$

96.0Zレ-

カたくてレ

996Zレ-

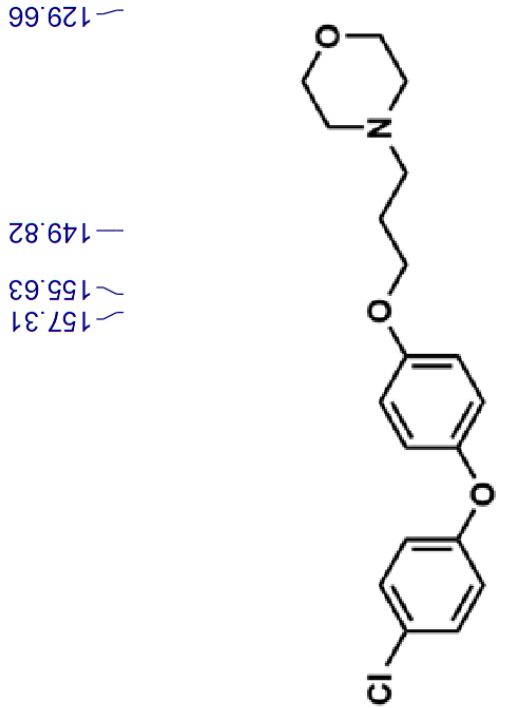


$\angle 9 \cdot 9$

69.9

$69^{\circ} 9$

$9 Z \mathrm{~L}$

$\nabla G^{\circ} L$

$\nabla G^{\circ} L$

$G G^{\circ} L$

$G 9 L$

$99^{\circ} \mathrm{L}$

$\angle G^{\circ} L$

$\angle G^{\circ} L$

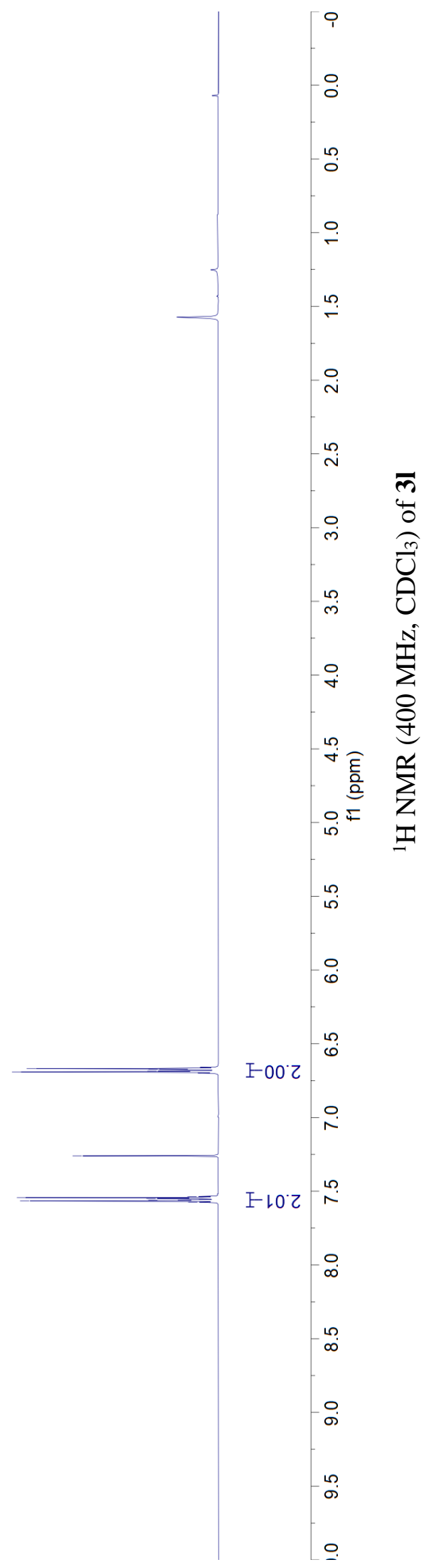




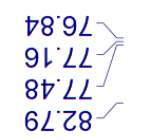

979レー

乙ย'8ยレ-

G969レ-

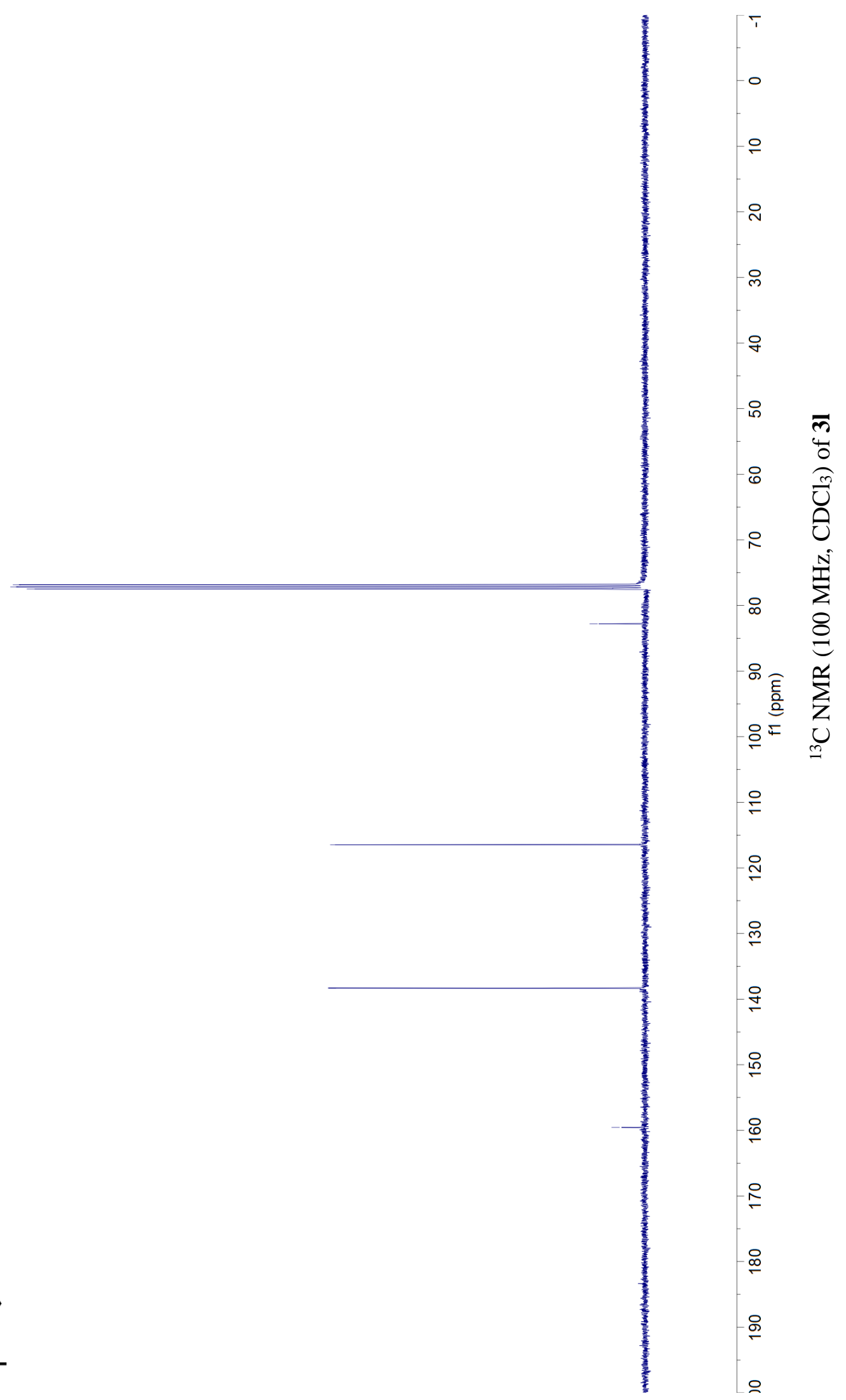



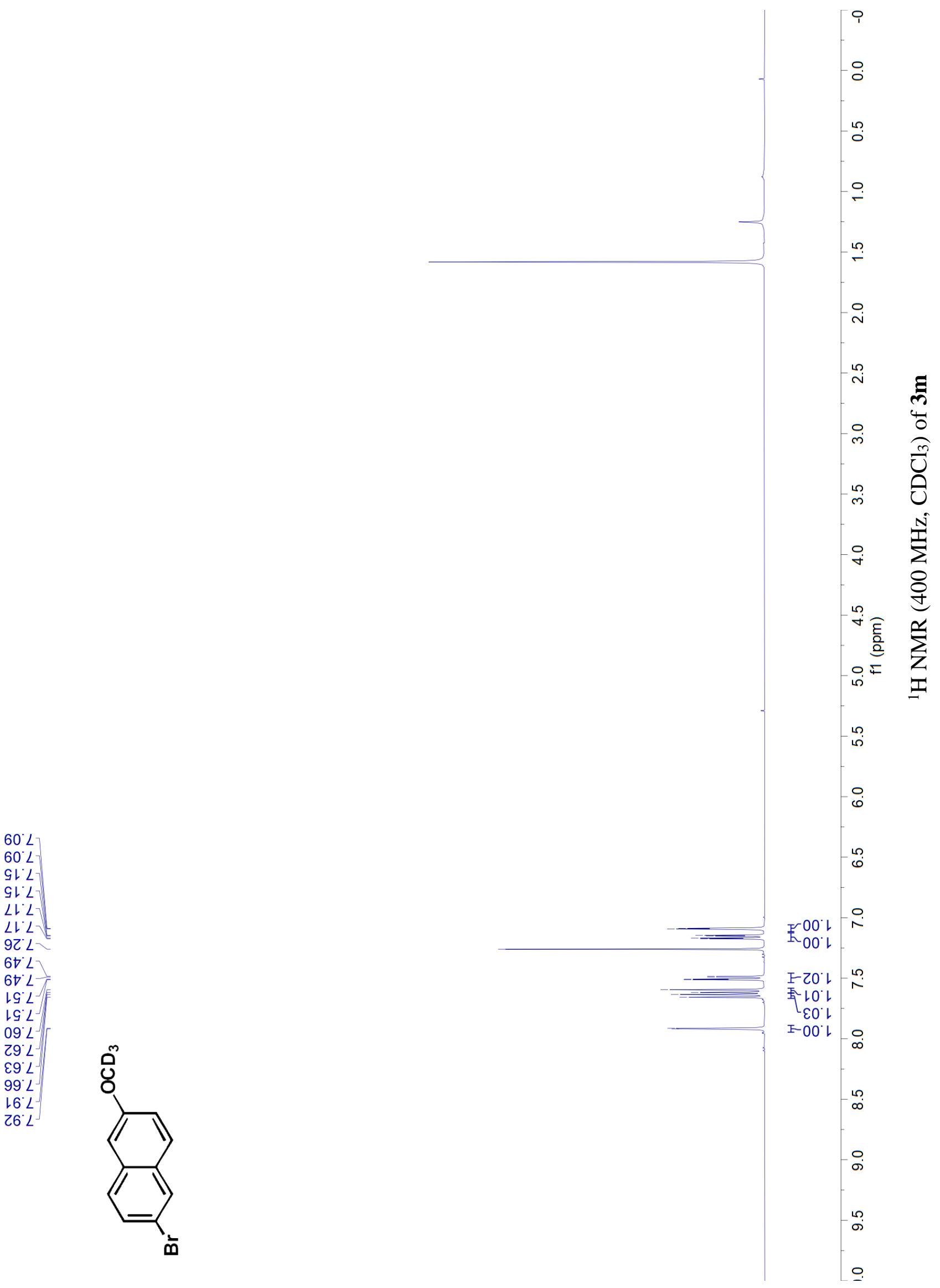


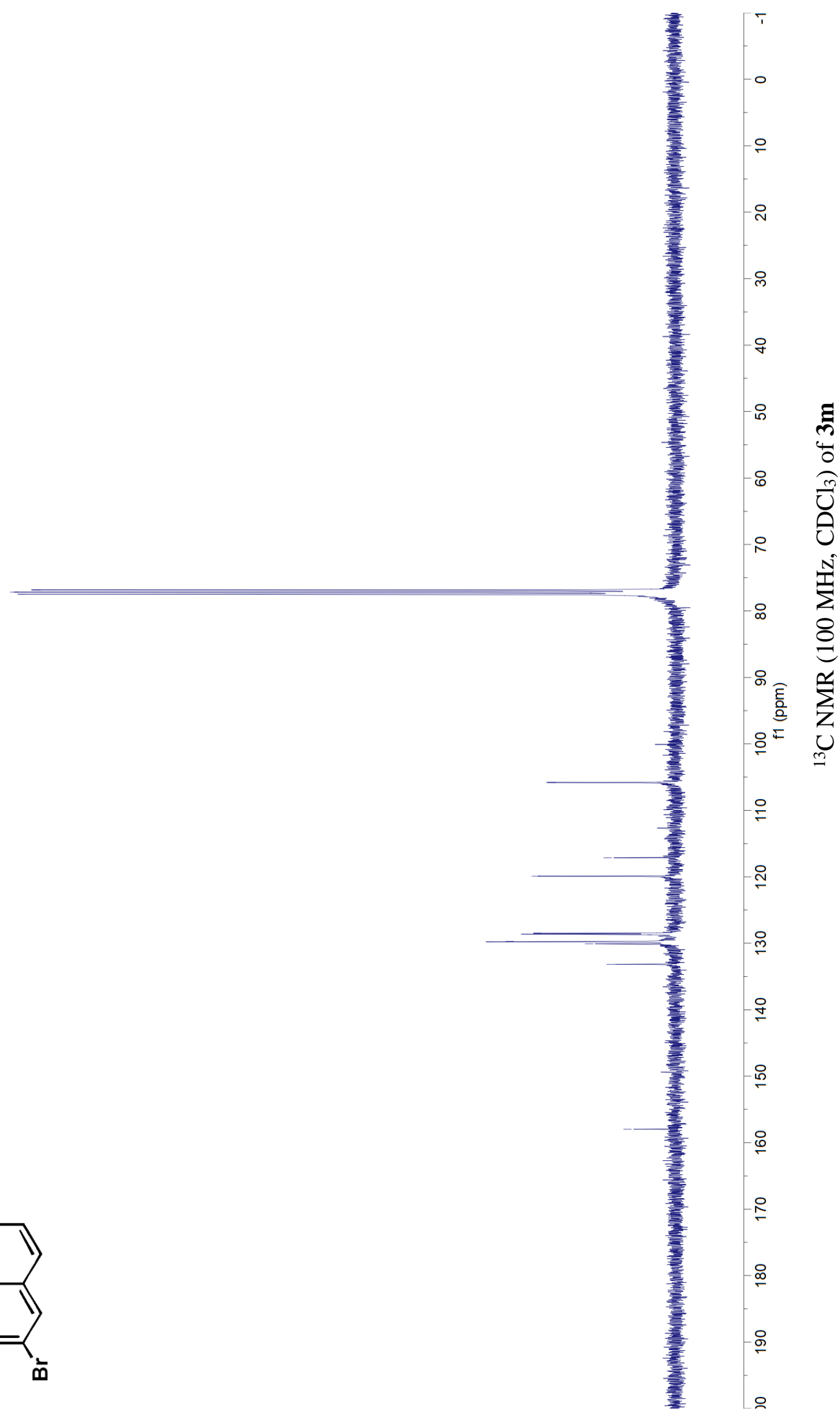

EL $\angle L L$

06.6 L -

0G.8ZL

\&9 $82 \mathrm{~L}$

$\varepsilon L^{\circ} 6$ ZL

LL'6ZL

$60^{\circ} 0 \varepsilon \mathrm{L}$

$96^{\circ} \angle G L$

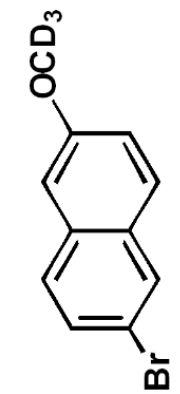




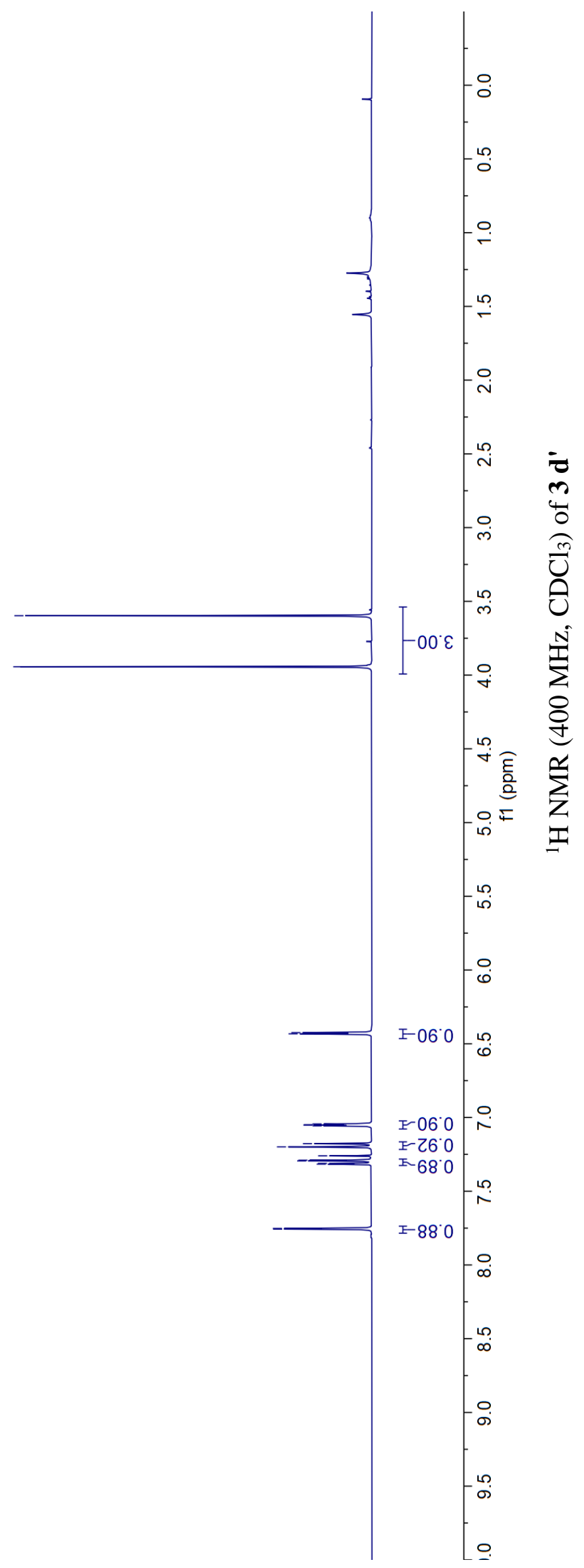




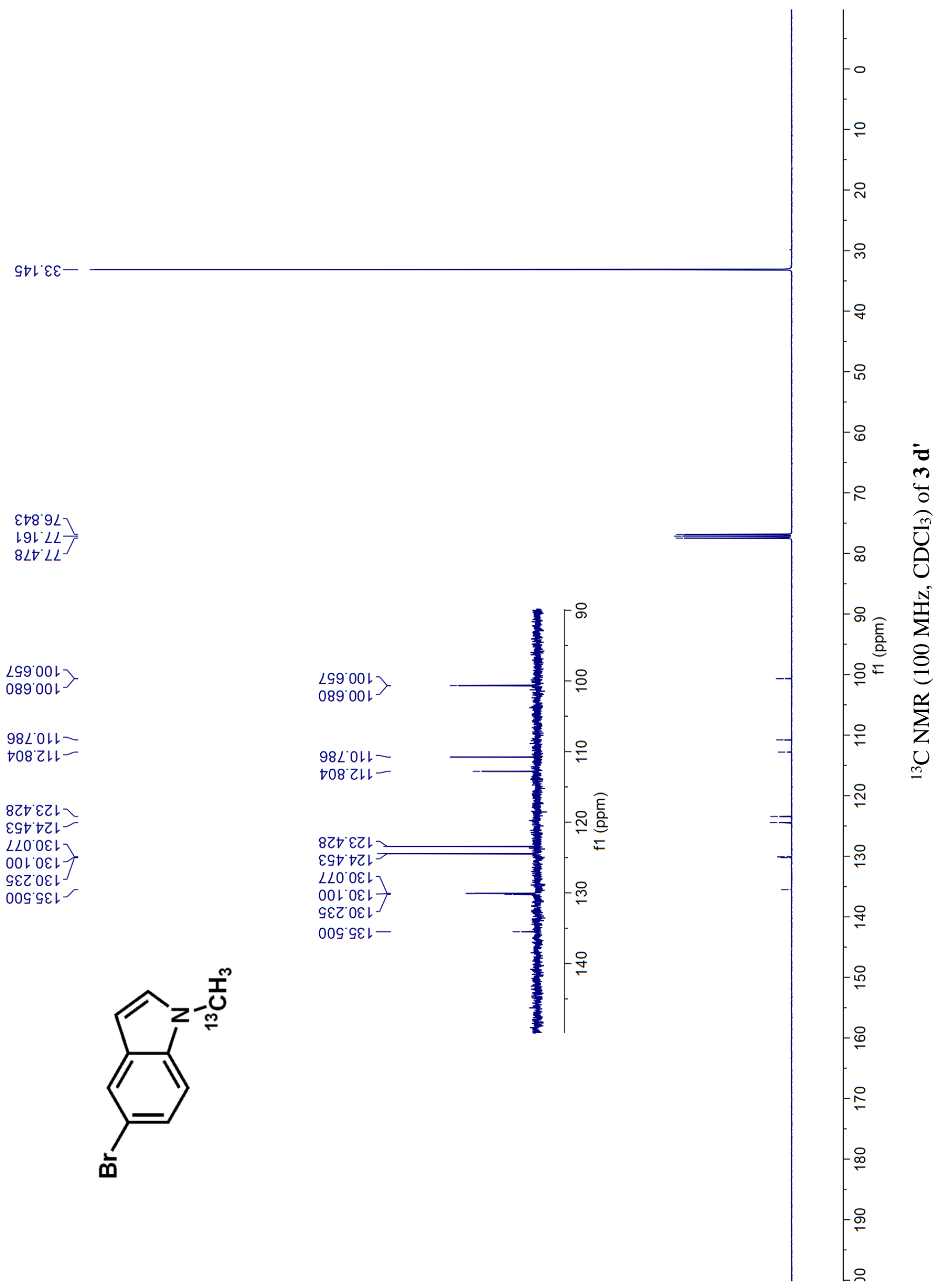



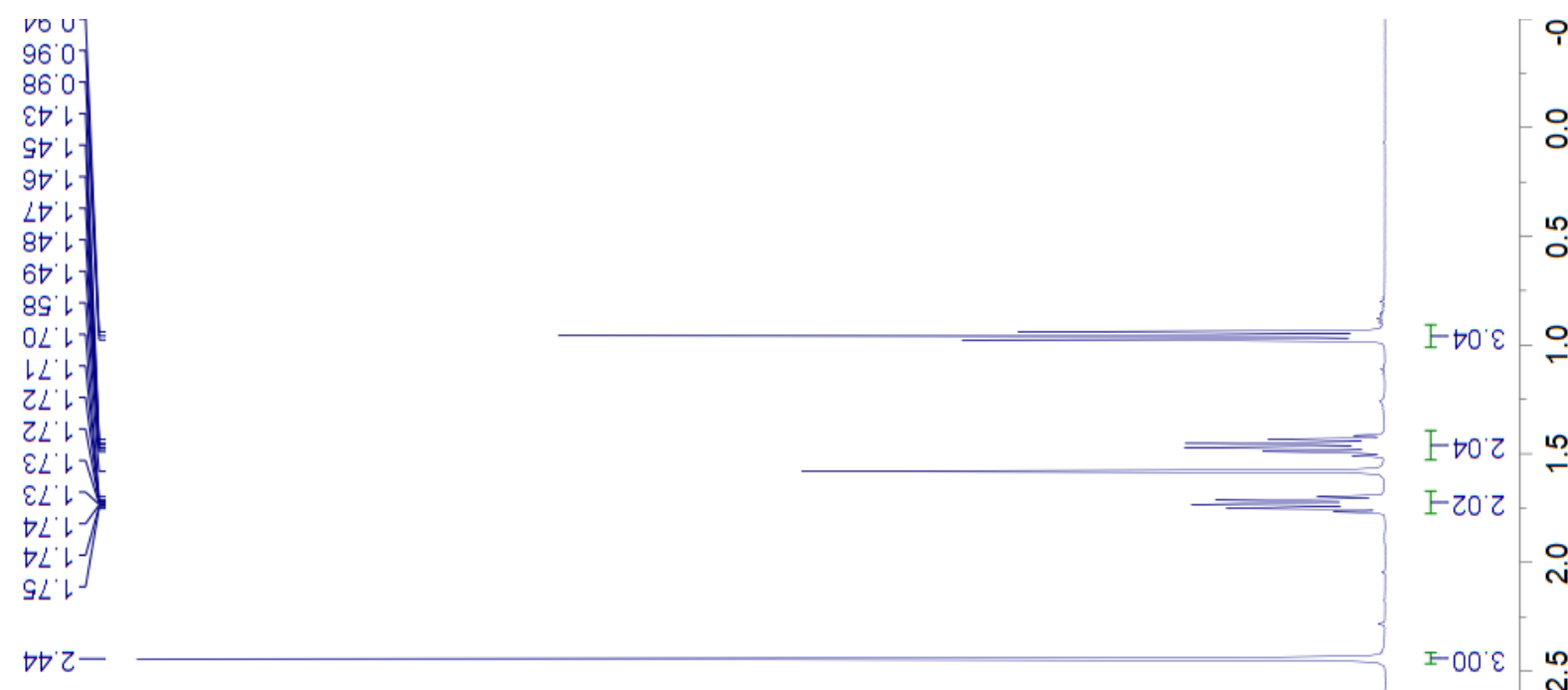

$88^{\prime} \varepsilon$

$68^{\prime} \varepsilon$

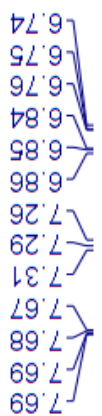

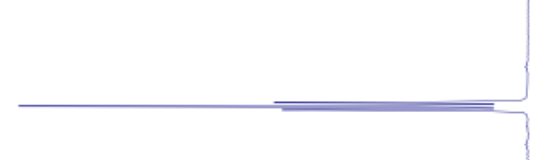

n

우

4
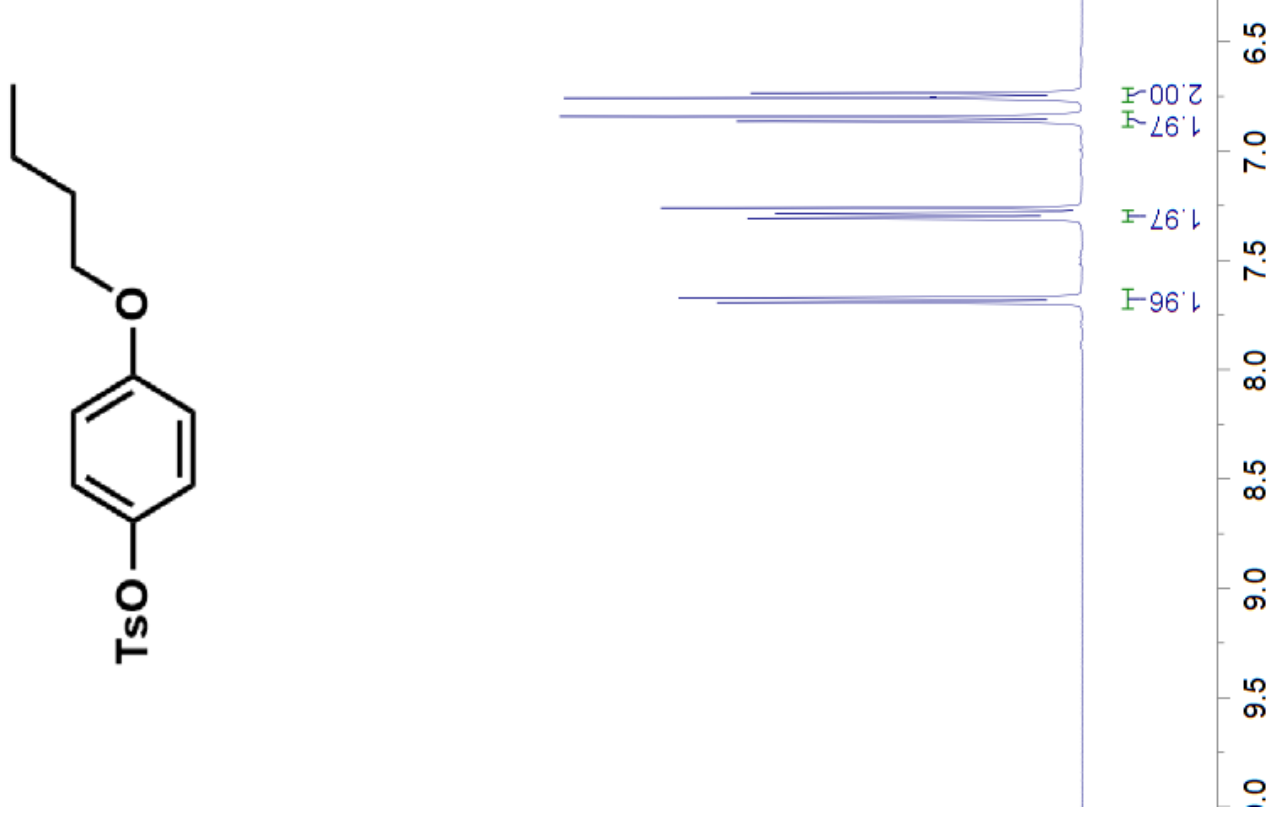


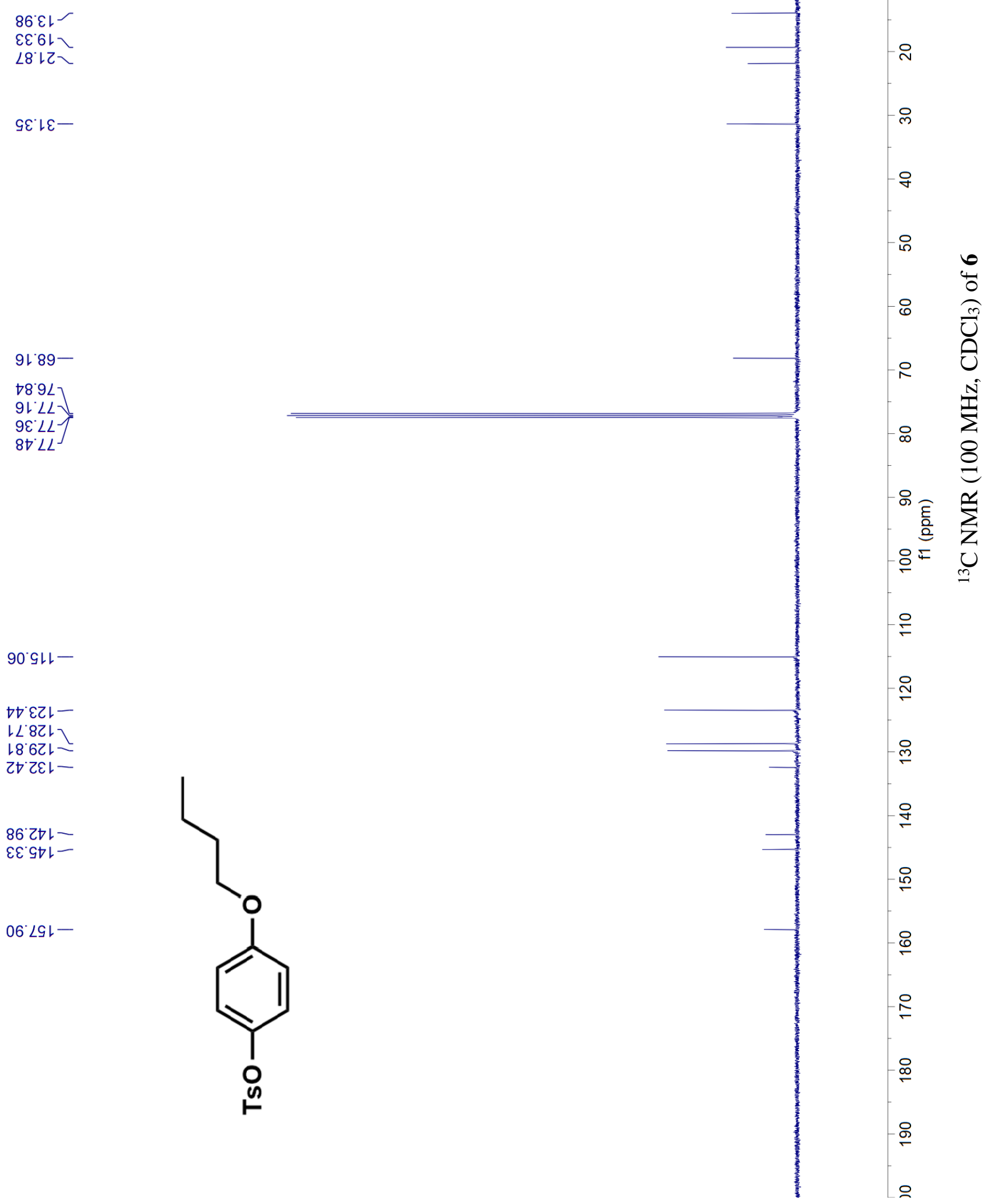



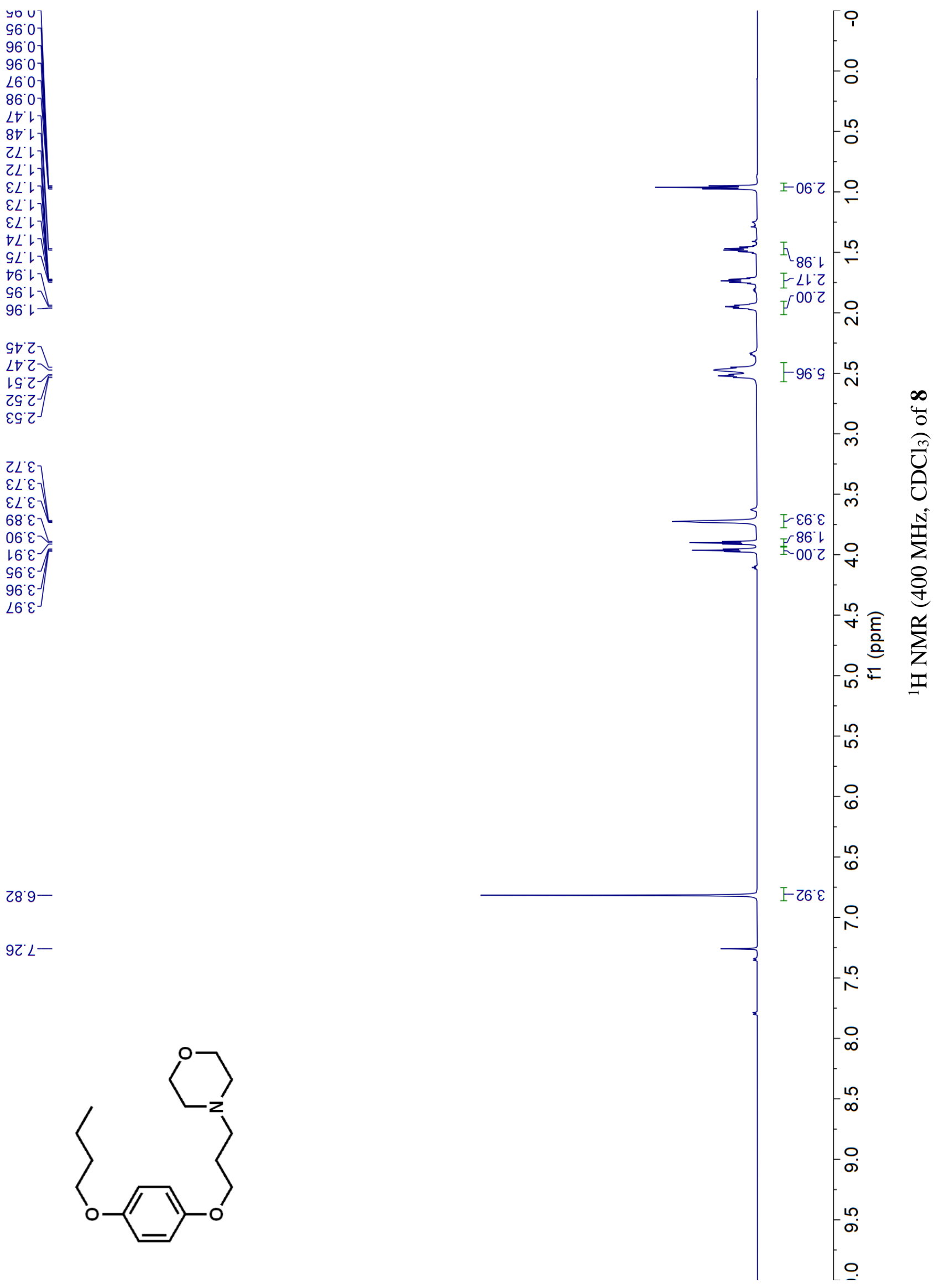


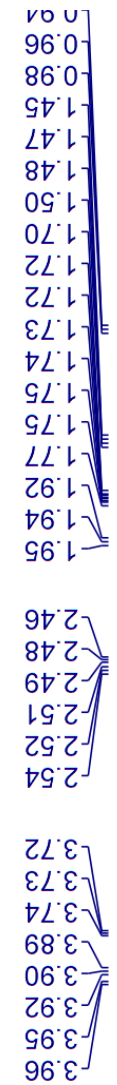

$289^{\circ-}$

$9 Z L-$

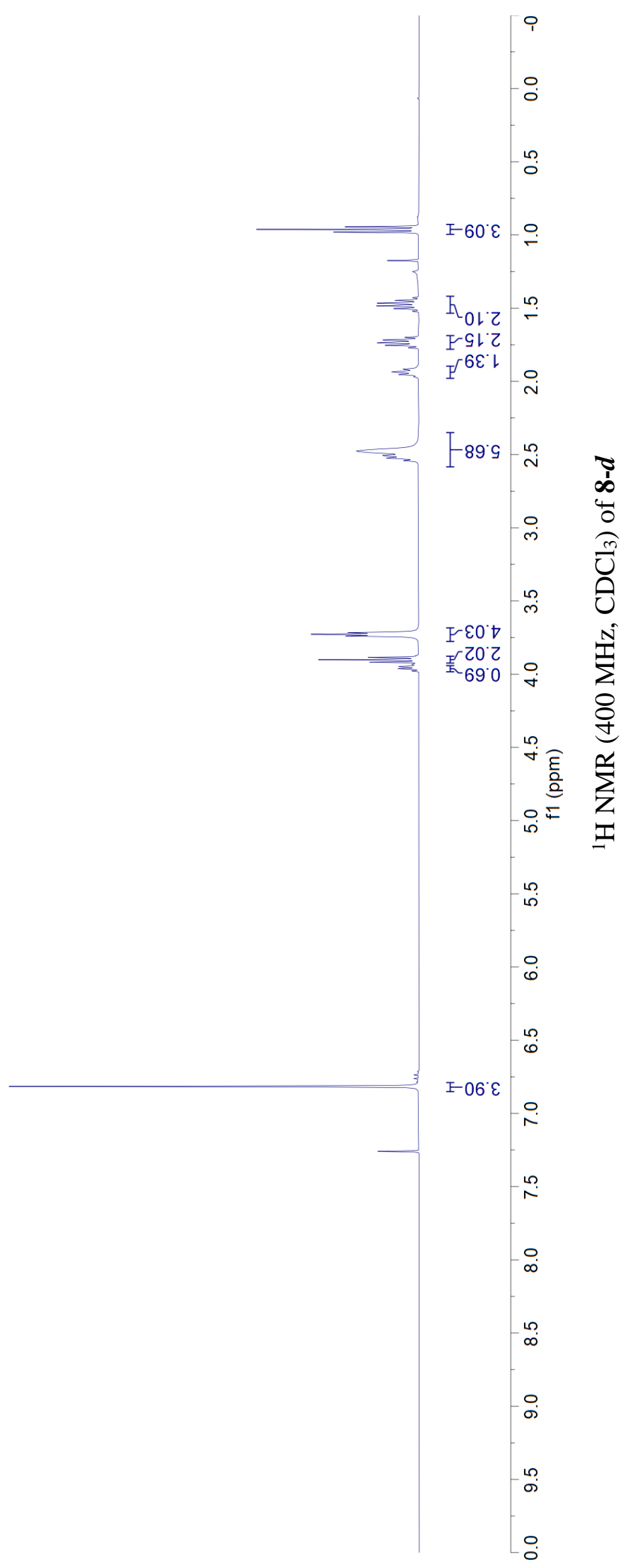




$$
\begin{aligned}
& \text { ยัจレー } \\
& \text { 6ย.6เ- } \\
& \text { เท92- } \\
& \angle G \cdot E-
\end{aligned}
$$

ง ${ }^{\circ}$ ต

$90^{\circ} \angle 9>$

乙カ89

†8 $9<7$

$912 L L$
$8{ }^{\circ} L L$

8tงเー

$80^{\circ} \varepsilon 9 レ$
เซ๕์
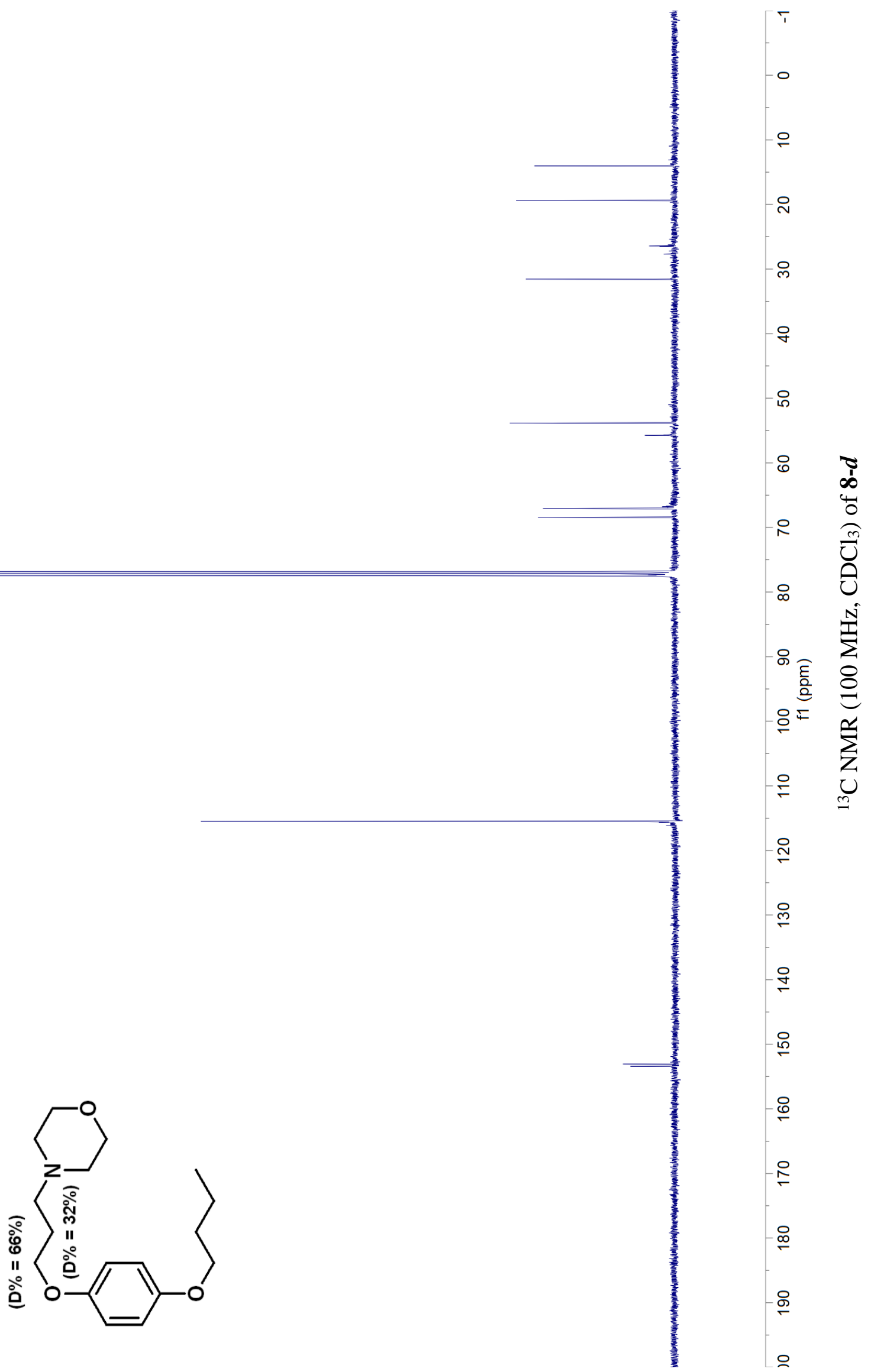